Andrews University

Digital Commons @ Andrews University

\title{
A Study of Perceived Actual Versus Expected Roles of Presidents of Seventh-day Adventist Senior Colleges and Universities in Eleven World Administrative Divisions
}

Sunith Kumar Das

Andrews University, dass@andrews.edu

Follow this and additional works at: https://digitalcommons.andrews.edu/dissertations

Part of the Higher Education Administration Commons, and the Leadership Studies Commons

\section{Recommended Citation}

Das, Sunith Kumar, "A Study of Perceived Actual Versus Expected Roles of Presidents of Seventh-day Adventist Senior Colleges and Universities in Eleven World Administrative Divisions" (2009). Dissertations. 1714.

https://digitalcommons.andrews.edu/dissertations/1714

https://dx.doi.org/10.32597/dissertations/1714

This Dissertation is brought to you for free and open access by the Graduate Research at Digital Commons @ Andrews University. It has been accepted for inclusion in Dissertations by an authorized administrator of Digital Commons@ Andrews University. For more information, please contact repository@andrews.edu. 
ABSTRACT

A STUDY OF PERCEIVED ACTUAL VERSUS EXPECTED ROLES OF PRESIDENTS OF SEVENTH-DAY ADVENTIST SENIOR COLLEGES AND UNIVERSITIES IN ELEVEN

WORLD ADMINISTRATIVE DIVISIONS

by

Sunith Kumar Das

Chair: Hinsdale Bernard 


\section{ABSTRACT OF GRADUATE STUDENT RESEARCH}

Dissertation

Andrews University

School of Education

Title: A STUDY OF PERCEIVED ACTUÁL VERSUS EXPECTED ROLES OF PRESIDENTS OF SEVENTH-DAY ADVENTIST SENIOR COLLEGES AND UNIVERSITIES IN ELEVEN WORLD ADMINISTRATIVE DIVISIONS

Name of researcher: Sunith Kumar Das

Name and degree of faculty chair: Hinsdale Bernard, Ph.D.

Date completed: December 2009

\section{Problem}

To date no study has been undertaken with respect to Seventh-day Adventist senior college and university presidents' actual and expected roles as perceived by the administrative team members and governing board chairs of these institutions. The purpose of this study was to determine the differences between presidents' actual and expected roles.

\section{Method}

This descriptive, exploratory, survey study included a sample $(n=78)$ of board chairs, presidents, academic vice presidents, student service vice presidents, and finance 
vice presidents located in 27 senior colleges and universities operated by Seventh-day Adventists in 17 countries, including the U.S.A. Data were collected on perceived actual roles and expected roles of presidents through a survey questionnaire adapted from Oliver (1975) and Silas (1974) containing 47 items. To test the three null hypotheses the instrument utilized descriptive statistics, one-way ANOVA, and a pairwise two-tailed $t$ test. To control for Type I error since there were many multiple comparisons, a corrected significance level was set at 0.001 using the traditional Bonferroni method.

\section{Results}

The results of this study showed that there were no statistically significant differences in perceptions among the five groups surveyed regarding actual and expected roles of presidents. Although each of the 47 expected roles was rated numerically higher than all the 47 actual roles, 31 comparisons showed significant differences. Among the topmost priorities placed on presidents' expected roles were: Focus on SDA Christian worldview; adhere to institution's educational philosophy; represent institution to churches; manifest Christ-like character; prepare agendas for board meetings; assist in fund-raising activities; be responsible for institution's strategic planning; and represent institution to public.

\section{Conclusions}

Focusing the SDA Christian worldview was the most prominent of all actual and expected roles. The groups shared a great deal of unanimity regarding their expectations of presidents' roles. The presidents were expected to perform much better compared to what were their actual performances in the areas of finance-related functions, fund-raising 
activities, and spiritual nurture programs. Recommendations for further research include an expansion of the scope comprising wider respondent groups to investigate their perceptions and to survey what could account for the wide disparities between their perceptions. It was also recommended to determine whether the low-scoring roles should be eliminated. 
Andrews University

School of Education

\title{
A STUDY OF PERCEIVED ACTUAL VERSUS EXPECTED ROLES OF PRESIDENTS OF SEVENTH-DAY ADVENTIST SENIOR COLLEGES AND UNIVERSITIES IN ELEVEN WORLD ADMINISTRATIVE DIVISIONS
}

\author{
A Dissertation \\ Presented in Partial Fulfillment \\ of the Requirements for the Degree \\ Doctor of Philosophy
}

by

Sunith Kumar Das

December 2009 
CCopyright by Sunith Kumar Das 2009 All Rights Reserved 


\title{
A STUDY OF PERCEIVED ACTUAL VERSUS EXPECTED ROLES OF PRESIDENTS OF SEVENTH-DAY ADVENTIST SENIOR COLLEGES AND UNIVERSITIES IN ELEVEN WORLD ADMINISTRATIVE DIVISIONS
}

\author{
A dissertation \\ presented in partial fulfillment \\ of the requirements for the degree \\ Doctor of Philosophy
}

by

Sunith Kumar Das

APPROVAL BY THE COMMITTEE:
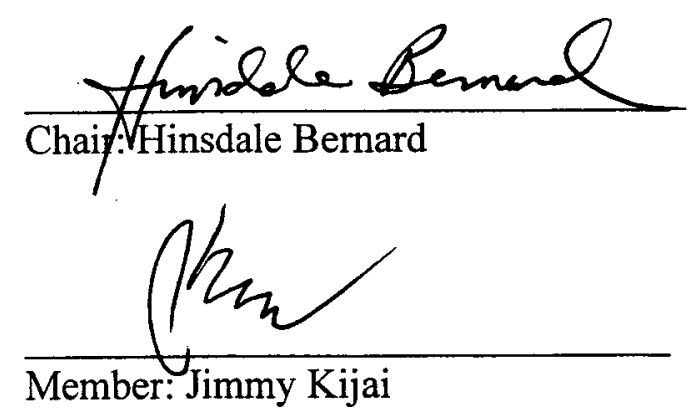

Member: Jimmy Kijai

Sylio Gouralez

Member: Sylvia Gonzalez

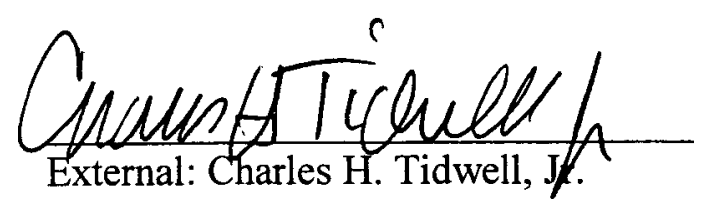

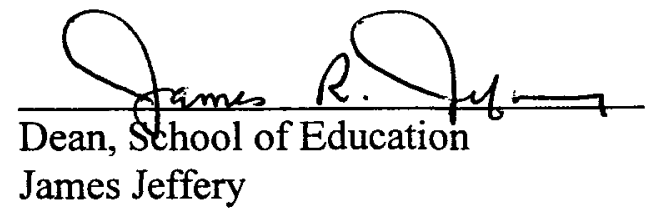

James Jeffery

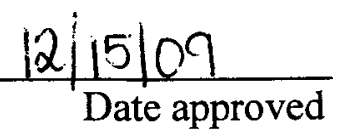




\section{To God,}

Who is the Source of life, ennobling knowledge and wisdom,

Who performed many miracles in my life

To my terrific dear wife, Millie, who encouraged me

to come to Andrews University from Singapore

to do my doctoral studies, and

who has supported me during the past years

To my wonderful children, Nellie and Steve, who understood my burden 


\section{TABLE OF CONTENTS}

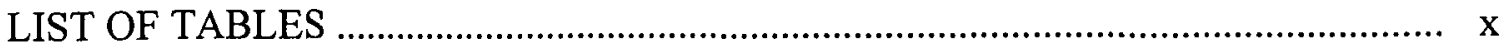

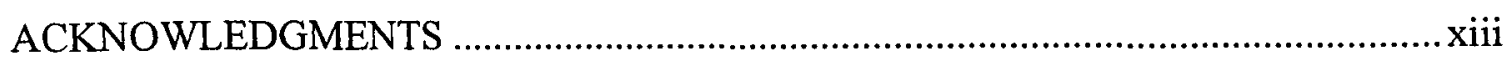

Chapter

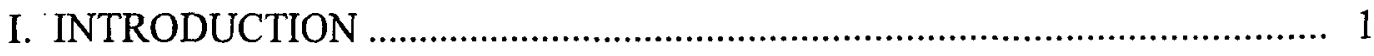

Background of the Study ..................................................................... 1

Statement of the Problem ...................................................................... 3

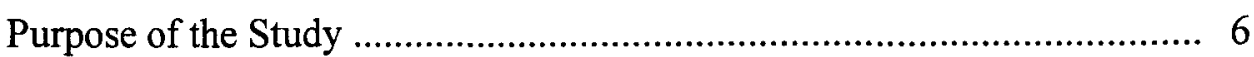

Introduction to Theoretical Concepts ........................................................ 6

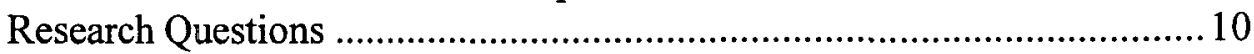

Research Hypotheses ......................................................................... 11

Rationale for the Study ...................................................................... 12

Significance of the Study ........................................................................ 14

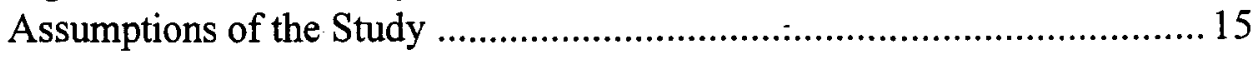

Delimitations of the Study ................................................................... 16

Limitations of the Study ...................................................................... 17

Definitions of Relevant Terms ........................................................... 18

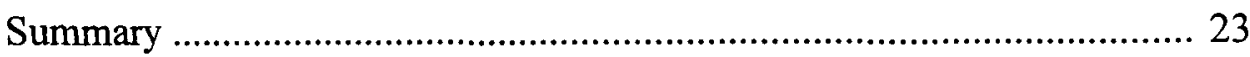

Organization of the Study .................................................................... 24

II. SELECTED REVIEW OF LITERATURE …............................................ 25

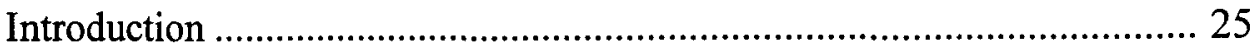

Historical Evolution of Higher Education and

College/University President's Roles ............................................. 26

Role Theory ..................................................................................... 30

Role Perception .......................................................................... 30

Role Expectation ........................................................................... 31

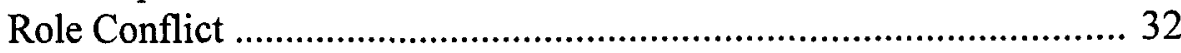

Related Studies on Public and Private College/University Presidency ... 32

Related Studies on Perceptions and Expectations of President's Roles .. 36

Broad Areas of President's Functions .................................................... 45 
Educational Program-Related Functions …………………..................... 46

Student-Related Functions .................................................................... 48

Finance- and Fund-Raising-Related Functions ........................................ 50

Public- and Alumni-Related Functions ................................................ 52

Faculty-Related Functions ………………………………................. 54

Physical Facilities-Related Functions ................................................... 56

Spiritual Life-Related Functions ........................................................... 58

Seventh-day Adventist College/University

President's Roles and Functions ......................................................6 60

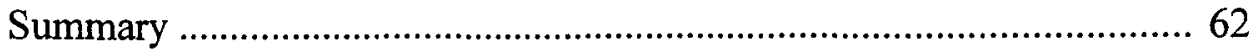

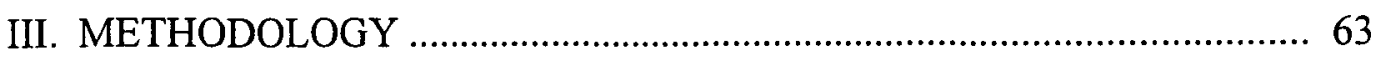

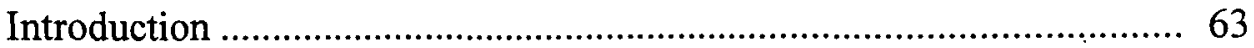

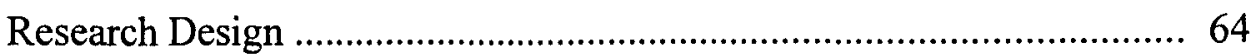

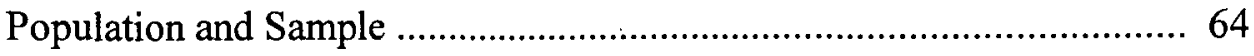

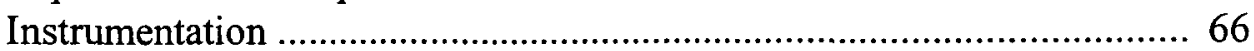

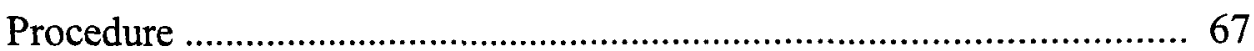

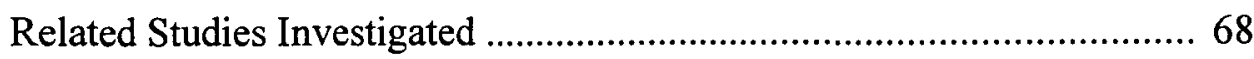

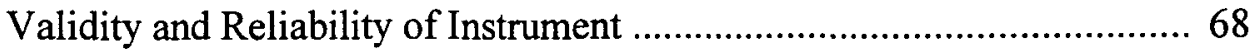

Preliminary Instrument .............................................................. 70

Modified Instrument ...................................................................... 72

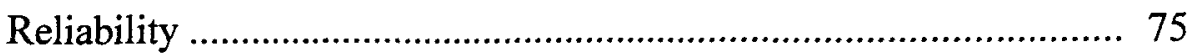

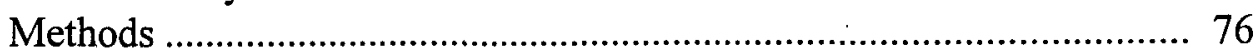

Collection of Data ............................................................................ 78

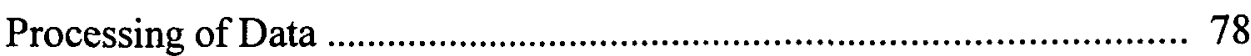

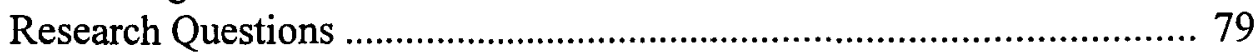

Related Null Hypotheses and Their Statistical Tests .............................. 80

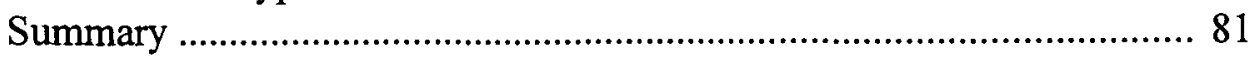

IV. PRESENTATION AND ANALYSIS OF DATA …….............................. 82

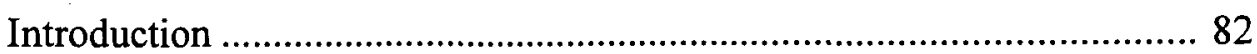

Sample Characteristics ....................................................................... 84

Data Analyses ............................................................................... 90

Research Questions 1 and 2 ............................................................. 107

Research Question 1 ...................................................................... 107

Very strong role performance ................................................... 11.1

Strong role performance ...............................................................111

Moderate role performance ...........................................................114

Weak role performance ..........................................................116

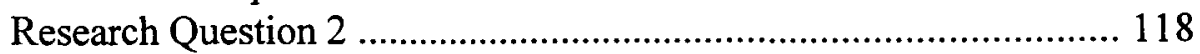

Very high role expectation ...................................................... 122 
High role expectation .................................................................. 123

Moderate role expectation ....................................................... 124

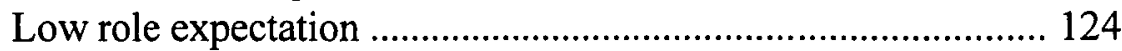

Research Questions 3, 4, and 5 with Related Null Hypotheses

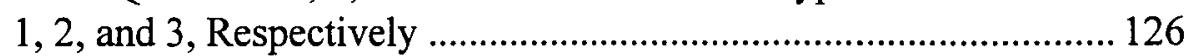

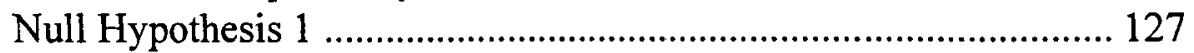

Null Hypothesis 2 ..................................................................... 129

Null Hypothesis 3 ....................................................................... 130

I. Student-Related Functions ................................................. 135

II. Employee-Related Functions ............................................... 138

III. General Administrative Functions ...................................... 140

IV. Finance-Related Functions ................................................ 142

V. Physical Facilities-Related Functions .................................. 143

VI. Community- and Alumni-Related Functions ....................... 145

VII. Spiritual Life-Related Functions ....................................... 146

Summary

\section{SUMMARY, FINDINGS, DISCUSSION, CONCLUSIONS} AND RECOMMENDATIONS

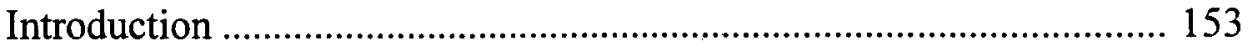

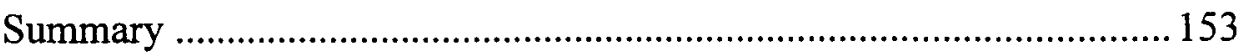

Background of the Study .......................................................... 153

The Problem ....................................................................... 154

Purpose of the Study ............................................................... 155

Brief Review of Literature ...................................................... 155

Research Methodology ………………………………........... 160

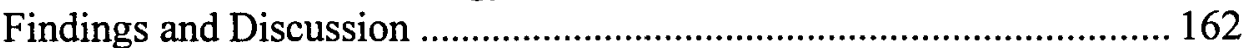

Research Question 1 ............................................................... 162

Research Question 2 .............................................................. 163

Findings and Discussion of Descriptive Statistics

in Relation to Research Questions 1 and 2 ............................... 163

I. Student-Related Functions .................................................164

II. Employee-Related Functions .................................................167

III. General Administrative Functions ..................................... 169

IV. Finance-Related Functions .............................................. 170

V. Physical Facilities-Related Functions ...................................172

VI. Community- and Alumni-Related Functions ........................173

VII. Spiritual Life-Related Functions ........................................175

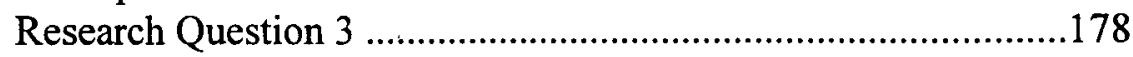

Research Question 4 ................................................................179

Findings and Discussion of Null Hypotheses 1 and 2 in Relation

to Research Questions 3 and 4, Respectively ............................. 179 
I. Student-Related Functions 180

II. Employee-Related Functions .................................................182

III. General Administrative Functions ...................................... 184

IV. Finance-Related Functions ................................................186

V. Physical Facilities-Related Functions ..................................188

VI. Community- and Alumni-Related Functions ........................190

VII. Spiritual Life-Related Functions .........................................192

Research Question 5 ................................................................194

Findings and Discussion of Null Hypothesis 3

in Relation to Research Question 5 ..........................................195

I. Student-Related Functions--Actual versus Expected ........... 195

No differences ............................................................... 196

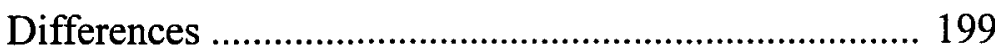

II. Employee-Related Functions--Actual versus Expected........202

No differences .............................................................. 202

Differences .................................................................... 205

III. General Administrative Functions--

Actual versus Expected ................................................ 210

No differences ............................................................ 210

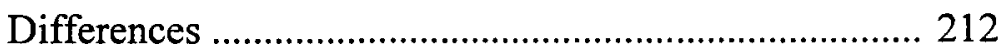

IV. Finance-Related Functions--Actual versus Expected ........ 220

Differences .................................................................. 220

V. Physical Facilities-Related Functions--

Actual versus Expected .................................................. 225

No differences ............................................................. 225

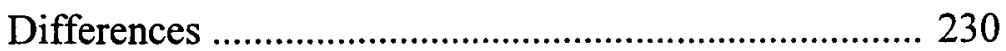

VI. Community- and Alumni-Related Functions--

Actual versus Expected ................................................. 232

Differences ...................................................................... 232

VII. Spiritual Life-Related Functions--

Actual versus Expected ................................................ 238

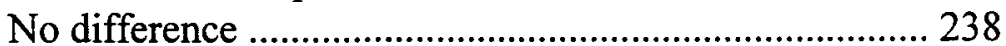

Differences .................................................................. 239

Final Comments on No Differences and Differences

Between Actual and Expected Roles ......................................... 241

No differences ………………………......................... 241

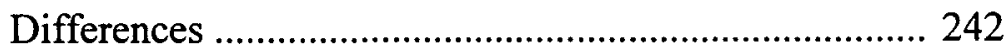

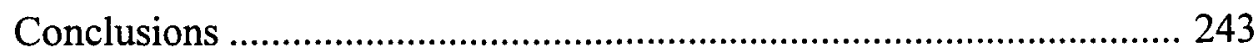

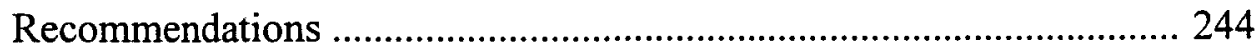

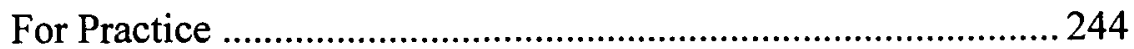

For Future Research …......................................................... 246

Appendix 
A. RESEARCH PROTOCOL

B. ENDORSEMENT OF THE STUDY 252

C. SAMPLE INFORMED CONSENT DOCUMENT 255

D. CHARACTERISTICS OF A SUCCESSFUL SEVENTH-DAY ADVENTIST COLLEGE OR UNIVERSITY

E. PRELIMINARY RESEARCH INSTRUMENT STUDY 262

F. MODIFIED RESEARCH INSTRUMENT AND INSTRUCTIONS 278

Questionnaire and Instructions for Presidents 279

Questionnaire and Instructions for Board Chair, Academic VPs, Student Service VPs, and Finance VPs 288

G. COMPOSITE ANOVA RESULTS (TABLES 33-39) FOR ACTUAL ROLES SHOWING MEANS AND STANDARD DEVIATIONS FOR SUB-HYPOTHESES 1-47

H. COMPOSITE ANOVA RESULTS (TABLES 40-46) FOR EXPECTED ROLES SHOWING MEANS AND STANDARD DEVIATIONS FOR SUB-HYPOTHESES 1-47

I. COMPARATIVE ONE-WAY ANOVA RESULTS (TABLES 47-53)

FOR ACTUAL AND EXPECTED ROLES

FOR SUB-HYPOTHESES 1-47

J. COMPARATIVE LEVELS OF PERCEIVED ACTUAL ROLE PERFORMANCES AND EXPECTED ROLE RESPONSIBILITIES OF PRESIDENTS IN RANK ORDER OF HIGHEST TO LOWEST MEANS

K. COMPARATIVE LEVELS OF PERCEIVED ACTUAL ROLE PERFORMANCES AND EXPECTED ROLE RESPONSIBILITIES OF PRESIDENTS BY CATEGORIES OF FUNCTIONS 338

L. RANKING OF FIVE STATUS GROUPS FOR THEIR PERCEPTIONS AND EXPECTATIONS OF SEVEN CATEGORIES OF ACTUAL AND EXPECTED FUNCTIONS 
M. RANKING OF SEVEN CATEGORIES OF ACTUAL AND

EXPECTED FUNCTIONS AS PERCEIVED

BY STATUS GROUPS

N. RANKING OF SIXTEEN ITEMS HAVING NO SIGNIFICANT

DIFFERENCES BETWEEN ACTUAL AND

EXPECTED ROLES

O. RANKING OF THIRTY-ONE ITEMS HAVING SIGNIFICANT STATISTICAL DIFFERENCES BETWEEN ACTUAL AND EXPECTED ROLES

BIBLIOGRAPHY 


\section{LIST OF TABLES}

1. World Divisions, Numbers of Countries and Colleges/Universities Represented

2. Internal Consistency Reliability Estimates of Role Scales ...................................76

3. Colleges/Universities and Countries Included in the Study by Divisions..............85

4. Participating Countries by Status Groups ..................................................... 86

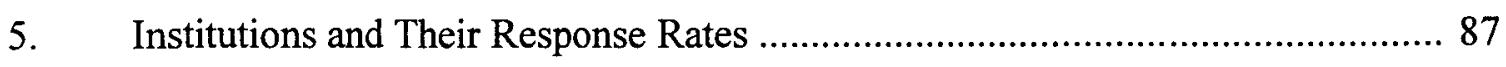

6. Respondents by Status Groups and Institutions ….......................................... 88

7. Summary of Participating Status Groups by Countries ........................................89

8. Means and Standard Deviations of Actual and Expected Roles:

I. Student-Related Functions

9. Means and Standard Deviations of Actual and Expected Roles:

II. Employee-Related Functions

10. Means and Standard Deviations of Actual and Expected Roles:

III. General Administrative Functions

11. Means and Standard Deviations of Actual and Expected Roles:

IV. Finance-Related Functions

12. Means and Standard Deviations of Actual and Expected Roles:

V. Physical Facilities-Related Functions 95

13. Means and Standard Deviations of Actual and Expected Roles:

VI. Community- and Alumni-Related Functions 96

14. Means and Standard Deviations of Actual and Expected Roles:

VII. Spiritual Life-Related Functions 
15. Summary of Means and Standard Deviations of Actual and Expected Roles: Tables 8-14

16. Items With Highest Ratings by Groups .......................................................... 99

17. Item Ratings by Board Chairs Higher Than All Groups ....................................100

18. Item Ratings by Presidents Higher Than All Groups ..........................................101

19. Item Ratings by Student Service VPs Higher Than All Groups .........................102

20. Item Ratings by Academic VPs Higher Than All Groups ................................. 102

21. Item Ratings by Presidents Having Lower Expected Means Than Actual Means

22. Item Ratings by Student Service VPs Having Lower Expected Means Than Actual Means

23. Item Ratings by Presidents Having Same Means and Same Standard

Deviations for Actual and Expected Roles

24. Items With Same Means for Actual and Expected Roles

But Different Standard Deviations

25. Groups' Perceptions of Presidents' Actual Role Performance

by Rank Order of Means

26. Summary Distribution of Number of Items by Levels of Actual Role

Performances and Categories of Functions

Based on Table 25

27. Groups' Perceptions of Presidents' Expected Role Responsibilities

By Rank Order of Means .

28. Summary Distribution of Number of Items by Levels of

Expected Role Responsibilities and Categories

of Functions Based on Table 27

29. Composite Two-Tailed $t$-Test Results Comparing Presidents' Actual

Role Performances and Expected Role Responsibilities

30. Comparisons by Percentages Between Actual and Expected Roles 148 
31. Summary Results of Null Hypotheses Tested 149

32. A Comparison of Actual and Expected Role Perceptions by Level 165 


\section{ACKNOWLEDGMENTS}

My doctoral journey is a memorable one. More than two decades ago Dr. George P. Babcock, then associate education director of the Seventh-day Adventist world church headquartered in Maryland, casually threw an idea to me of coming to Andrews University to pursue a Ph.D. course in Educational Administration. Since then I have been cherishing this dream in my heart. I thank Dr. Babcock for inspiring me to do doctoral studies.

During my years of study here at Andrews University I came across some stalwarts who have been a great help to me. Although they are no longer at Andrews University, the names that come to my mind are Late Dr. Wes Shultz, former dean of College of Technology; Late Dr. Bernard Lall, former professor of Educational Administration and Supervision; Dr. Loretta Johns, former assistant dean of College of Arts and Sciences; Dr. Edward Streeter, former chair of the Department of Educational Administration and Supervision; Dr. Richard Orrison, former professor of Teaching and Learning; Dr. Robert Moon, former acting dean of the School of Graduate Studies; and Dr. Lisa Beardsley, former dean of the School of Graduate Studies at Andrews University, and current executive secretary of the Accrediting Association of Seventh-day Adventist Schools, Colleges, and Universities (AAA) at the General Conference. They provided me teaching and research jobs that helped me academically and professionally. 
All professors from whom I took classes made an immense impact in my academic pursuit. Among those who aroused critical thinking in my learning experiences were: Dr. George Akers, Dr. Paul Brantley, Dr. Lyndon Furst, Dr. Wilfred Futcher, Dr. Jimmy Kijai, Late Dr. Bernard Lall, Dr. Robert Schwab, Dr. Edward Streeter, and Dr. James Tucker.

Special thanks are due to my dissertation committee that has guided me through my long Ph.D. journey. I am profoundly grateful to Dr. Hinsdale Bernard for his willingness to serve as chair of my dissertation committee, after Dr. Lyndon Furst, my former chair, left the School of Education to become Dean of the School of Graduate Studies at Andrews University. In his meticulosity, thoroughness, super knowledge, high intellect, and mentoring skill, Dr. Bernard guided me in every step of this dissertation writing. He called me on the phone and sent me e-mails enquiring about the progress and giving me advice. I am forever indebted to him for his professional help.

Dr. Jimmy Kijai, a committee member, helped me on the research methodology, while Dr. Sylvia Gonzalez, another committee member, gave me advice on the content. I consider their feedback to be very important. I thank them for their valuable input.

I thank the General Conference Education Department of the Seventh-day Adventist World Church in Maryland for endorsing this study. Especially, I would like to recognize Dr. Humberto Rasi, former education director of the General Conference; Dr. Garland Dulan, current education director; and Dr. John Fowler, current associate education director of the General Conference of the Seventh-day Adventist world church, for showing their interest in this research. 
I received a total of twenty-seven faculty handbooks from twenty-seven colleges and universities around the world. Out of these, eleven faculty handbooks came from the college/university campuses in North America. The rest were from countries on the continents of Africa, Asia, Australia, Europe, North America, and South America. I owe special thanks to the senders of these documents that contained job descriptions of the presidents, vice presidents, and other administrators of the colleges and universities.

I want to reiterate my profound appreciation to all the participants from sixteen countries in eleven world divisions, including the fifteen instrument validation members, for participating in this international research project. Dr. Jim Jeffrey, dean of the School of Education, Dr. Shirley Freed, chair of the Department of Leadership and Educational Administration, and all the Graduate Educational Program Committee members deserve special thanks for granting me the needed time to complete this global study. I want to express my appreciation to Bonnie Proctor, dissertation secretary, for taking the time to go through this huge document, ensuring that it meets the required format and style.

My wife, Millie, was an immense inspiration, encouragement, and strength to me. I will ever remain indebted to her for the support she has provided me by her hard work, prayers, and shouldering many burdens while I was in school. I am also thankful to my children, Nellie and Steve, for being so understanding. My family has been a big support to me--financially and emotionally.

Most importantly, my ever-growing gratitude is to my God, my never-failing Friend, who, in His amazing grace, has marvelously carried me through during the past few years. Without His help this dissertation could not have been completed. 


\section{CHAPTER I}

\section{INTRODUCTION}

\section{Background of the Study}

The Seventh-day Adventists operate one of the largest Protestant school systems in the world. ${ }^{1}$ Beginning with the first Seventh-day Adventist school ${ }^{2}$ in Battle Creek, Michigan, in 1872, the church's educational program has expanded into a huge international network. According to the latest Annual Statistical Report ${ }^{3}$ of the General Conference of Seventh-day Adventists, a total of 7,284 educational institutions were in operation in the 2005-2006 academic year of which 5,666 were primary schools, 1,512 secondary schools and worker training institutes, and 106 colleges and universities with a total of $1,436,290$ students and 75,183 teachers in its system. The Seventh-day Adventist Yearbook ${ }^{4}$ published by the world headquarters of the Seventh-day Adventist church, makes mention of 33 universities and senior colleges in operation around the world that

'Bernard Mohan Lall and Geeta Rani Lall, New Dynamic Leadership: How to Become an Effective Leader in a Contemporary Society (Berrien Springs, MI: Geetanjali Publishers, 1994), 4.

${ }^{2}$ Meredith Jones Gray, "Battle Creek College 1874-1901," in Christ in the Classroom: Adventist Approaches to the Integration of Faith and Learning, ed. Humbarto M. Rasi (Silver Spring, MD: Institute for Christian Teaching, 1999), 24:138; see also Allan G. Lindsay, "God Used a Man: Goodloe Bell-Faithful Friend to Right in Every Form," Adventist World-NAD 4, no. 8 (2008): 35.

${ }^{3}$ General Conference of Seventh-day Adventists, $144^{\text {th }}$ Annual Statistical Report-2006 (Silver Spring, MD: Office of Archives and Statistics, 2006), 6.

${ }^{4}$ Seventh-day Adventist Yearbook 2008 (Hagerstown, MD: Review \& Herald, 2008), 443-494. 
offered one or more graduate degree programs under their own authorities since 20042005 "with projections of 103,500 students by 2010." These institutions are identified by the Accrediting Association of Seventh-day Adventist Schools, Colleges, and Universities, also referred to as Adventist Accrediting Association (AAA). ${ }^{6}$

According to Walton Brown, the object of Seventh-day Adventist senior colleges is to provide for the young men and women "a balanced education of the spiritual, physical, and intellectual powers, and to train them as workers for all branches of gospel service." ${ }^{7}$ The administrators and teachers need to work together in accomplishing this broad objective. Without a well-defined role on the part of each educator on campus this goal cannot be reached.

The president is the head of the institution and chief executive officer ${ }^{8}$ of the governing board of the institution, in that he or she executes the decisions of the board. The president should make plans and provide guidance under the direction of the board. The senior college or university president is expected to perform his or her role in order to help achieve the institutional goals.

The term "chief executive" is preferred as a generic term in the context of executives of Seventh-day Adventist institutions of higher learning worldwide who come

5Ella Simmons, "Aiming Higher," Adventist World 5, no. 7 (2009): 10; see also Garrett Caldwell, "Atlanta 2010: Seventh-day Adventist Church," Lake Union Herald 102, no. 1 (2010): 30.

${ }^{6}$ General Conference of Seventh-day Adventists, General Conference Working Policy, 2007-2008 ed. (Hagerstown, MD: Review \& Herald, 2008), 261-262.

${ }^{7}$ Walton J. Brown, A Handbook for Seventh-day Adventist School Administrators (Takoma Park, MD: Department of Education, General Conference of Seventh-day Adventists, 1980), 63.

${ }^{8}$ Harold W. Dodds, The Academic President: Educator or Caretaker? with the collaboration of Felix C. Robb and R. Robb Taylor (New York, NY: McGraw-Hill, 1962), 3. 
under various titles, such as, "president," "chancellor", "vice-chancellor," "rector," and "principal." Also, in this study the words "Seventh-day Adventist," "SDA," and "Adventist" are used interchangeably.

\section{Statement of the Problem}

The Seventh-day Adventist university and senior college presidents are the institutional leaders called upon to perform many functions. A senior college offers primarily four-year undergraduate programs, while a university offers undergraduate and graduate programs, including doctoral studies. These institutions are accredited by the Adventist Accrediting Association which does not have any well-defined job descriptions for the chief executives. The General Conference Education Department suggested ${ }^{9}$ that research be done on the roles and functions of Seventh-day Adventist college presidents. All that the Education Department of the world church has is a list of twelve characteristics of a successful Seventh-day Adventist college or university (Appendix D). This list was sent to all SDA institutions of higher education in the twelve world divisions at the time for administrators to make sure that these characteristics were met.

Role expectations of senior college or university presidents as viewed by academic deans, department administrative heads, teaching faculty, and board chairs vary from one educator to another. It appears that there is a lack of consensus on the

${ }^{9}$ In 1995, the Office of the Department of Education at the world headquarters of the General Conference of Seventh-day Adventists, Maryland, sent out a list of suggested topics for academic research in Adventist Education, to various Seventh-day Adventist colleges and universities around the world. One of the many topics included in the document was the "Role of the college/university president in the new century" that appeared under the section title, Administration. This study is a result of this topic selected from that list. Copy of this list is placed in my personal file. 
president's role. According to Silas, when these expectations are the same, it is expected that the president can do his or her duties without conflict. ${ }^{10}$ When these expectations do not agree, the president is in a position of conflict.

Many questions, challenges, and uncertainties arise regarding the president's role and function. How should the senior college or university president function? Should he or she try to satisfy all the status groups despite their differences in matters of his or her role expectations? Or, should the president disregard the groups and pursue his or her own expectations based on personal perceptions? If conflict develops, has it developed due to dissimilarity in the way the president and the reference groups perceive the president's role expectations? How does the president perceive his or her functions? Does the ideology of the five groups under study, such as board chairs, presidents, academic vice presidents, student service vice presidents, and finance vice presidents, differ among themselves? If so, what are the differences? How do the president, academic dean, chief financial officer, dean of students, and board chair believe the president should function? Is there a correlation between what the senior college or university president does and what he or she should do as perceived by the groups?

It would be useful to know what these status groups expect from their presidents and also what the presidents expect from their administrative team members. One problem is in the area of job description and actually determining what it is that the presidents are supposed to do. Therefore, it is important that their position be examined.

\footnotetext{
${ }^{10}$ Samuel L. Silas, "Role Expectations of Presidents in Predominantly Black State and Privately Supported Colleges and Universities as Perceived by Trustees, Presidents, and Deans" (Ph.D. dissertation, Southern Illinois University, 1974), 3.
} 
The extensive search of the related literature provided no record of studies on the role and function of the presidents of the Seventh-day Adventist institutions of higher learning worldwide. I looked at nine studies that are briefly presented in chapter 2 . Of these, two dissertations ${ }^{11}$ dwelt on the expectations and perceptions regarding presidents' roles in selected public, private, and parochial universities and colleges. The main features of these two studies are briefly presented in chapters 2 and 3 of this dissertation.

The role theory enunciated by Stogdill, ${ }^{12}$ Chase,${ }^{13}$ and Campbell, ${ }^{14}$ as summarized by Clark, ${ }^{15}$ indicates that almost any activity of any person may be considered as being in conformity with, or in opposition to, the expectations of his or her role. The expectations include not only the individuals' own concept of their role but also the expectations of others pertaining to their behavior in the role which they perform. In an effort to partially determine these expectations, this study focused upon the dimensions of leader behavior. An analysis of perceptions of the chief executive' officer's role may be of significant importance in studying leadership in Seventh-day Adventist institutions of higher

"Silas, "Role Expectations," and Arnold Robert Oliver, "The University Presidency as Perceived by Boards of Trustees, Presidents, and Academic Vice Presidents in Selected Colleges and Universities" (Ph.D. dissertation, Southern Illinois University, 1972).

${ }^{12}$ Ralph M. Stogdill, Ellis L. Scott, and William E. Jaynes, Leadership and Role Expectations (Columbus, OH: Bureau of Business Research, Ohio State University, 1956), quoted in Lita Allen Clark, "Role Expectations for the Chief Executive Officer of a University System" (Ed.D. thesis, University of Alabama, 1977), 4.

${ }^{13}$ Francis S. Chase, "How to Meet Teachers' Expectations of Leadership," Administrative Handbook (1953), quoted in Clark, 4.

${ }^{14}$ Roald F. Campbell and Russell T. Gregg, Administrative Behavior in Education (New York, NY: Harper \& Bros., 1957), quoted in Clark, 5.

${ }^{15}$ Clark, 10. Clark's study focused on the role expectations of the university system's chief executive officer and examined the working relationships existing among the selected institutional chief executives and the university system's chief executive in a university system. 
education. The research problem behooved the purpose of this study.

\section{Purpose of the Study}

The purpose of this study is threefold: Purpose 1 is to determine the actual role performances of presidents of Seventh-day Adventist senior colleges and universities around the world offering graduate programs on their campuses. Purpose 2 is to determine the expected roles of presidents of SDA senior colleges and universities around the world offering graduate programs on their campuses. Finally, Purpose 3 is to determine the differences between actual and expected roles of presidents of SDA senior colleges and universities around the world offering graduate programs on their campuses. These purposes are to answer the five research questions of this study, and the underlying theoretical concepts provide a basis to pose these relevant research questions.

\section{Introduction to Theoretical Concepts}

It is essential to discuss the theoretical concepts of role perception and role expectation because the studies regarding senior college and university presidents are grounded in administrative theory. Development of organizational behavior of closed and open systems has gone through several stages since 1910. Richard Scott classifies these emerging administrative theories into three competing systems perspectives, such as rational-systems, natural-systems, and open-systems. ${ }^{16}$ These three views are relatively distinct, yet they are partly overlapping, partly complementary, and partly conflicting in

\footnotetext{
${ }^{16} \mathrm{~W}$. Richard Scott, Organizations: Rational, Natural, and Open Systems, $5^{\text {th }}$ ed. (Upper Saddle River, NJ: Prentice-Hall, 2003), 20-116. Scott, professor emeritus in the Department of Sociology, focused on the general issues of institutional influences on organizational forms and functions.
} 
their approaches. Each has its antecedents in earlier concepts.

The rational-systems model has its roots in 1911, when Frederick Taylor, the father of the scientific management, formulated the "machine" theory, believing that workers could be programmed to be efficient machines. ${ }^{17}$ The administrator's role under this model could be motivated by goals, division of labor, specialization, standardization, hierarchy, span of control, exception principle, and formal organization. Perhaps the greatest shortcoming of machine theory is its rigid conception of the organization. ${ }^{18}$

During the 1930s inadequacies were perceived in the rational-systems model and, in contrast, another theory, known as natural-system or organic model, was introduced. The natural-systems perspectives had its early roots in the human relations approach. Beginning in the early 1950 s, educational theorists moved from the technical problems of administration to the human aspect of administrative problems. Mary Parker Follett was a pioneer in the human relations movement. ${ }^{19}$ According to the natural-systems perspective or organic model theory, the human side of administration was the fundamental problem in all organizations. The role of the president is not purely a technical matter, but that it involves the human mind and its ability to conceptualize roles and functions. In 1955, Bauer indicated a similar concept when he spoke of a gradual

\footnotetext{
${ }^{17}$ Frederick Winslow Taylor and Frank B. Gilbreth, The Principles of Scientific Management, Primer of Scientific Management (Easton, PA: Hive Publishing Co., 1985), 49-51. Gilbreth answered questions regarding Taylor's "machine" concept that was applied to employees under scientific management.
}

${ }^{18}$ Wayne K. Hoy and Cecil G. Miskell, Educational Administration: Theory, Research, and Practice, $6^{\text {th }}$ ed. (New York, NY: McGraw-Hill, 2001), 10-14.

${ }^{19}$ Mary Parker Follett, Creative Experience (New York, NY: Longmans, 1924), p. ix-xiii, quoted in Hoy and Miskell, 14. 
shift from administrators' emphasis on command and control functions to what may be termed evocative and coordinative functions, releasing individuals and providing the most fertile environment for the employees. ${ }^{20}$ Ten years later, Getzels discussed these same ideas on a more sophisticated level. Daniel Griffiths, the founder of the National Conference of Professors of Educational Administration (NCPEA), states that subsequent research by such foundations as the W. K. Kellogg Foundation of Battle Creek, Michigan, contributed much to the study and the perception of roles in administration. ${ }^{21}$

The open-systems model came into existence as a reaction to the rational-systems approach and natural-systems approach. It has the potential to provide a synthesis by combining the rational and natural perspectives. Colleges and universities are open systems confronted with both rational and natural constraints that change as the environmental forces change; to neglect either the rational or the natural elements is shortsighted. The open-systems perspective is the overarching framework that underscores four internal subsystems that interact to influence presidents' behaviors: the structural, cultural, individual, and political systems. ${ }^{22}$

People within an institution have varying and often conflicting expectations of how the chief executive should perform. ${ }^{23}$ The presidents will have some concept of their $18-25$.

${ }^{20}$ Ronald C. Bauer, Cares in College Administration (London, England: Teachers College, 1959),

${ }^{21}$ Jacob W. Getzels, James Lipham, and Roald F. Campbell, Educational Administration as a Social Process (New York, NY: Harper \& Row, 1968), 4 \& 5, quoted in Oliver, 17 \& 18.

${ }^{22} \mathrm{Hoy}$ and Miskell, 18-20.

${ }^{23}$ Bruce J. Biddle and Edwin J. Thomas, eds., Role Theory: Concepts and Research (New York, NY: John Wiley, 1966), 33. 
own roles, but it is very important that they take into consideration the expectations of other colleagues on campuses. They should also receive feedback from their administrative team members and support staff as to how they themselves are performing their jobs.

According to Stogdill, the true leadership role is determined by the expectations of the leader and of other team members directly involved ${ }^{24}$ Chase indicated that leaders' roles are compounded by the demands of the situation; the leaders' own concept of their roles; and the expectations of the people they work with. ${ }^{25}$ Campbell and Gregg stated, "Role conflict exists when the expected administrator behavior, as defined by one group, is different from the role as perceived by the administrator himself or by other groups. ${ }^{126}$

In every campus this situation exists, whether public, private, parochial, or Adventist institutions of higher education. According to Hunt, the concept of role has been identified as "demand" or influences brought upon an individual making him behave in ways that others expect him to behave. ${ }^{27}$ Katz and Kahn had a similar concept. They wrote:

Associated with each office is a set of activities or expected behaviors. These activities constitute the role to be performed, at least approximately, by any person who occupies that office.

Generally, role refers to the recurring actions of an individual,

${ }^{24}$ Stogdill, Scott, and Jaynes, 2, quoted in Clark, 4.

${ }^{25}$ Chase, quoted in Clark, 4.

${ }^{26}$ Campbell and Gregg, 345, quoted in Clark, 5.

${ }^{27}$ Larry W. Hunt, "A Study of the Perceptions of Presidents and Academic Deans Regarding Role Expectations and Decision-Making Styles of Academic Deans in Texas Community Colleges" (Ph.D. dissertation, North Texas State University, 1977), 3. 
appropriately inter-related with the repetitive activities of others so as to yield a predictable outcome. The set of interdependent behaviors comprise a social system or subsystem, a stable collective pattern in which people play their parts. ${ }^{28}$

One could, then, assume that the chief executives, or presidents of institutions of higher education, would behave as the academic deans, chief financial officers, deans of students, board chairmen, and other administrative team members expect them to perform. Thus it would follow that it would be of interest and benefit to determine what others included in the study expect from the presidents, and also what the presidents themselves expect to do on their jobs.

In the light of the theoretical concepts discussed it is appropriate to pose the following five research questions that are aligned with the purposes of this study.

\section{Research Questions}

To achieve the purpose of this study, the following five research questions were posed to guide the collection and analysis of data:

Question 1. What are the actual role performances of presidents of Seventh-day Adventist senior colleges and universities around the world, as perceived by their board chairs, presidents, academic vice presidents, student service vice presidents, and finance vice presidents?

Question 2. What are the expected role responsibilities of presidents of Seventhday Adventist senior colleges and universities around the world, as viewed by their board chairs, presidents, academic vice presidents, student service vice presidents, and finance

\footnotetext{
${ }^{28}$ Daniel Katz and Robert L. Kahn, The Social Psychology of Organizations (New York, NY: John Wiley, 1966), 10, quoted in Hunt, 3.
} 
vice presidents?

Question 3. Is there a difference in perception between groups of board chairs, presidents, academic vice presidents, student service vice presidents, and finance vice presidents of Seventh-day Adventist senior colleges and universities around the world regarding their college/university presidents' perceived actual role performances?

Question 4. Is there a difference in expectation between groups of board chairs, presidents, academic vice presidents, student service vice presidents, and finance vice presidents of Seventh-day Adventist senior colleges and universities around the world regarding their college/university presidents' expected role responsibilities?

Question 5. Is there a difference in perception and expectation between actual role performances and expected role responsibilities of presidents of Seventh-day Adventist senior colleges and universities around the world, as perceived and expected by their groups of board chairs, presidents, academic vice presidents, student service vice presidents, and finance vice presidents?

\section{Research Hypotheses}

The following research hypotheses were suggested to guide the study on actual versus expected roles:

Hypothesis 1. There is a difference in perception between status groups of board chairs, presidents, academic vice presidents, student service vice presidents, and finance vice presidents of Seventh-day Adventist senior colleges and universities around the world on each of the actual role performances of their college/university presidents. 
Hypothesis 2. There is a difference in expectation between status groups of board chairs, presidents, academic vice presidents, student service vice presidents, and finance vice presidents of Seventh-day Adventist senior colleges and universities around the world on each of the expected role responsibilities of their college/university presidents.

Hypothesis 3. There is a difference between perception and expectation between actual role performances and expected role responsibilities of presidents of Seventh-day Adventist senior colleges and universities around the world, as viewed by each of the status groups of board chairs, presidents, academic vice presidents, student service vice presidents, and finance vice presidents.

\section{Rationale for the Study}

The president or whatever title he or she may hold as chief executive officer is the head of the college or university. Brown maintains that it is the president who should have the responsibility of making plans and providing guidance under the orientation of the board of trustees. ${ }^{29}$ The president has a wide variety of duties and is responsible for everything that happens within the institution. While it is true that he or she carries the total responsibility, he or she will utilize all the abilities of his or her fellow-workers in doing the work. He or she will delegate responsibility to them, allowing them to carry the load. To do this, there should be clearly defined job descriptions for Seventh-day Adventist senior college and university presidents. The General Conference Education Department office does not have any formal job description for the Seventh-day

\footnotetext{
${ }^{29}$ Brown, 126.
} 
Adventist senior college or university president. ${ }^{30}$

In March of 1995, the General Conference's Education Department had circulated to all the graduate schools worldwide a list of suggested research subjects ${ }^{31}$ pertinent to contemporary Adventist higher education. From this list I chose the subject, entitled, "Role of the College/University President in the New Century." I modified this subject to the topic under study as presented in this research document. There has not been any study done so far in this area. The Education Department of the world church of the Seventh-day Adventists has endorsed this study (Appendix B) and considered it to "be valuable for current and future Adventist college and university presidents. ${ }^{132}$

The academic community of the twenty-first century requires a challenging leadership role to meet the needs of these changing times. Hence, this study included the leadership functions as expected by the members of the administrative teams of Adventist institutions of higher education.

The General Conference's Education Department, which oversees the Seventhday Adventist university and senior college operation around the globe, ${ }^{33}$ has sparked this research. No study has been done so far on the perceived roles and functions of chief

\footnotetext{
${ }^{30}$ The General Conference's Department of Education of the Seventh-day Adventist world church wrote to me of this study: "We do not have in our department a job description for the position of president of an Adventist college or university." Copy of this letter is in my personal file.

${ }^{31}$ See n. 9.

${ }^{32}$ The General Conference's Department of Education of the Seventh-day Adventist world church wrote to me: "I was very pleased to learn through your letter that you have embarked in the preparation of your doctoral dissertation and that you selected one of the subjects suggested by our department. I think that the topic is relevant and that it will be valuable for current and future Adventist college and university presidents." Copy of this letter is in my personal file.
}

${ }^{33}$ General Conference Working Policy, 2007-2008 ed., 250. 
executives of Adventist higher educational institutions worldwide, as expected by various administrative team members. Hence, there is a great need to embark on this inquiry.

\section{Significance of the Study}

This study of the actual and expected roles of the presidents of the Seventh-day Adventist senior colleges and universities worldwide is significant for several reasons.

First, from its humble beginning of the first SDA college in 1874 in Battle Creek, Michigan, ${ }^{34}$ the Seventh-day Adventists have operated more than one hundred institutions of higher education since 2005 , worldwide, when the survey for this study was conducted. ${ }^{35}$ Seventh-day Adventist colleges and universities are on the increase. ${ }^{36}$ Society is becoming more and more pluralistic. Technology is advancing in leaps and bounds. The human factor is becoming complicated. New demands arise in all aspects of life as time rolls in the twenty-first century. In the background of all this, higher education faces a great challenge. Seventh-day Adventist education also faces a similar situation. The educational chief administrators need to understand their roles and functions in ever-changing times and circumstances, so that they can optimally carry out their responsibilities.

Second, this study will help to identify the areas of agreement and disagreement

\footnotetext{
${ }^{34}$ Floyd Greenleaf, "Timeline for Seventh-day Adventist Education," The Journal of Adventist Education 67, no. 5 (2005): 10.

${ }^{35}$ Department of Education, General Conference of Seventh-day Adventists, "World Education Statistics December 31, 2004," The Journal of Adventist Education 67, no. 5 (2005): 2.

${ }^{36}$ Department of Education, "Seventh-day Adventists and Scholarship," The Journal of Adventist Education 67, no. 5 (2005): 16-17. This is a revision of a paper prepared by the initial writing committee for the International Conference on Faith and Science, held in Denver, Colorado, in August 2004.
} 
between the presidents and their administrative teams on the matter of the presidents' actual role performances and expected role responsibilities as chief executive officers of the educational institutions of the Seventh-day Adventist church worldwide. It can help the college and university administrators in their effective administration.

Third, this study is noteworthy because I am not aware of any past or existing scholarly studies designed to investigate the perceived role performance and expected role responsibilities of chief executive administrators at Seventh-day Adventist senior colleges and universities worldwide that offer graduate programs on their campuses.

There have been some studies conducted on the role of chief academic officer and chief financial officer, but there is a very clear need for several studies of this kind to be done on the president, who is the chief executive officer of the Seventh-day Adventist institutions of higher education.

\section{Assumptions of the Study}

For the purposes of this study, the following assumptions are made:

1. There would be certain functions common to all presidents regardless of the size of the institution, although organizational size could affect degree of consensus in role perception and role expectation. ${ }^{37}$

2. The differences in role expectations and role perceptions can be identified. ${ }^{38}$

3. When differences in president's role expectations and role perceptions are

\footnotetext{
${ }^{37}$ Theodore Sarbin and Vernon L. Allen, "Role Theory," in The Handbook of Social Psychology, ed. Gardner Lindzey and Elliot Aronson, $2^{\text {nd }}$ ed., vol. 1 (Reading, MA: Addison-Wesley, 1968), 504.

${ }^{38}$ Silas, 11.
} 
understood, misunderstandings can be more readily resolved. ${ }^{39}$

4. When president, academic dean, chief financial officer, dean of students, and board chairperson agree on the president's role and functions, the conflict is minimal. ${ }^{40}$

5. The survey population has a good insight into the president's functions and is most suited to provide the information to be sought. Their answers to the questionnaire are objective and comparable.

\section{Delimitations of the Study}

This study has the following delimitations:

1. This study was limited to twenty-eight Seventh-day Adventist senior colleges and universities around the world that offered graduate programs on their campuses under their own authorities in the 2004-2005 school year as identified in the Seventh-day Adventist Yearbook. The remaining seventy-one non-graduate colleges were not included.

2. Information on the perceived and expected role and function of the president was gathered from senior college and university presidents, academic vice presidents, student service vice presidents, finance vice presidents, and board chairs. The general faculty and staff, student population, board members, parents, and alumni were not included in this study. The actual process, implementation, and results or effects of previous administrative roles were not studied, but an effort was made to assess current functions and expected role responsibilities, as reported by respondents.

\footnotetext{
${ }^{39}$ Ibid., 11.

${ }^{40}$ Sarbin and Allen, 540-544.
} 
3. Since the size and type of institutions could make a difference in the role of the president, ${ }^{41}$ the study was limited to institutions that have enrollments of more than 200 .

4. The participating senior colleges and universities offering graduate programs in 2004-2005 on their campuses were selected from the tertiary-level (G), one of the three classifications ${ }^{42}$ made by the General Conference's Education Department as follows:

G--A tertiary-level institution offering one or more graduate degree programs under its own authority.

C--A tertiary-level institution offering one or more baccalaureate degrees under its own authority.

JC--A tertiary-level institution offering less than four years of postsecondary studies (not leading to a baccalaureate degree).

Institutions of the above $\mathrm{G}$ classification ${ }^{43}$ accredited by the Adventist Accrediting Association were selected for this study.

\section{Limitations of the Study}

This study was subject to the following limitations:

1. This study was dependent upon mailed questionnaires for collection of information and is therefore subject to limitations in adequacy of communication. Parten rightly says that the respondent may misinterpret questions, omit essential items, or submit material which cannot be tabulated, thus making it necessary to discard many of

\footnotetext{
${ }^{41}$ See n. 37.

${ }^{42}$ Seventh-day Adventist Yearbook 2008, 443-495.

${ }^{43}$ Ibid., 405 .
} 
the questionnaires. ${ }^{44}$

2. Since this study was global in nature and was dependent upon mailed questionnaires which were subject to less than 100 percent returns due to postal handling and other reasons, there was a lack of information about those who failed to respond.

3. This study included voluntary respondents from five status groups, such as, board chairs, presidents, academic vice presidents, student service vice presidents, and finance vice presidents.

\section{Definition of Relevant Terms}

The following terms are defined as used in this dissertation:

Role: A pattern of behavior of those holding particular status position. ${ }^{45}$

Function: A part of a process, action, or operation that belongs to or is charged to a particular person in order that the overall operation may be accomplished. ${ }^{46}$

Role Expectation: Role expectation as used in this study and applied to the senior college/university president refers to the duties and qualities expected of him or her as he or she occupies the main leadership position on campus. ${ }^{47}$

Expected Role: The set of expectations for the behaviors of object persons that

${ }^{44}$ Mildred Parten, Surveys, Polls, and Samples (New York, NY: Harper \& Brothers, 1950), 95: see also Bernard M. Lall, "Role Expectations of the School Superintendent as Perceived by Superintendents, Principals, Teachers, and Board Members in the Province of Saskatchewan" (Ph.D. dissertation, University of Oregon, 1967), 7.

${ }^{45}$ E. Mark Hanson, Educational Administration and Organizational Behavior, $5^{\text {th }}$ ed. (Boston, MA: Allyn \& Bacon, 2003), 54.

${ }^{46}$ Leroy A. Wilch, "Perceived Leadership Role and Function of Nebraska Community College Presidents and Campus Directors" (Ph.D. dissertation, University of South Dakota, 1986), 9.

${ }^{47}$ Silas, 12. 
are consensually held by one or more subject persons. ${ }^{48}$

Role Perception: A personal disposition which causes varying degrees of positive or negative reactions toward one's role as measured by the responses to the items of the questionnaires. $^{49}$

Seventh-day Adventist (SDA): The official name of a Protestant Christian denomination that places strong emphasis on the Second Advent of Christ, which it believes is near, and observes the seventh day of the week as Sabbath. ${ }^{50}$ Seventh-day Adventist is abbreviated as SDA.

General Conference of Seventh-day Adventists (GC): The highest administrative body of the world church of the Seventh-day Adventists. Its major function is to coordinate the Seventh-day Adventist work worldwide. ${ }^{51}$ It is headquartered at Silver Spring, Maryland, U.S.A.

Division: "Divisions are sections of the General Conference, with administrative responsibility assigned to them in designated geographical areas ${ }^{152}$ consisting of particular countries as determined by the General Conference. There are thirteen

${ }^{48}$ Bruce J. Biddle, Role Theory: Expectations, Identities, and Behaviors (New York, NY: Academic Press, 1979), 387.

${ }^{49}$ J. P. Guilford, Psychometric Methods (New York, NY: McGraw-Hill, 1954), 4; see also Robert Albert Kalangi, "A Comparative Study of the Perception of the Principal's Role as Held by Teachers and Principals in the Seventh-day Adventist Academies in the United States" (Ph.D. dissertation, University of Nebraska, 1975), 3.

${ }^{50}$ Seventh-day Adventist Encyclopedia, 1976 ed., s.v. "Seventh-day Adventist."

${ }^{51}$ General Conference, Seventh-day Adventist Church Manual, $17^{\text {th }}$ ed. (Hagerstown, MD: Review \& Herald, 2005), 26.

${ }^{52}$ Ibid., 26. 
divisions $^{53}$ in the world that form the General Conference, the highest governing body of the Seventh-day Adventist world church.

Union Conference/Union Field/Union Mission: The union conference or union field or union mission is a united body of local conferences or local fields or local missions within a territory of a country or countries belonging to a division. ${ }^{54}$ There are ninety-four union conferences or union fields or union missions in 203 countries where there is a presence of SDA work. ${ }^{5 s}$

Status Group: "A specific group within a given social structure is usually referred to as a status group." ${ }^{156}$ This study includes five status groups. They are: (1) chairs of boards of trustees, or boards of directors, or boards of management, or college council, or university council, (2) presidents, or chancellor, or vice-chancellor, or rector, or principal, (3) vice presidents for academic affairs/administration, or academic deans, or provosts,(4) vice presidents for student services/affairs/life, or deans of students, or directors of student services, and (5) vice presidents for finance, or business managers, or directors of finance, or deans of finance.

President: The president of a college or university is its chief executive officer (CEO), leader, and official spokesman of the institution of higher education. He or she receives from the governing boards powers commensurate with his or her

${ }^{53}$ Seventh-day Adventist Yearbook 2008, 20-21.

${ }^{54}$ See n. 51 .

${ }^{55}$ Seventh-day Adventist Yearbook 2008, 4; see also Garret Caldwell, "Atlanta 2010: Seventh-day Adventist Church," Lake Union Herald 102, no. 1 (2010): 30.

${ }^{56}$ Lall, "Role Expectations," 13. 
responsibilities. ${ }^{57}$ In some institutions he or she is also called vice chancellor, chancellor, rector, or principal.

Academic Vice President: He or she serves as the chief advisor to the president in matters of instruction and research. He or she is usually called vice president for academic administration, or academic dean. In two cases of this study this position is titled as senior vice president for academic administration. In some institutions this position is also called vice chancellor for academic affairs. ${ }^{58}$ Sometimes provost, or provice chancellor, deputy vice chancellor, or executive vice president, or registrar carries on this responsibility. He or she is the chief academic officer (CAO) of the institution.

Finance Vice President: He or she is the chief financial officer of the senior college and university and is known with the acronym, CFO. He or she is usually called the vice president of finance, or vice president for financial administration. In two cases of this study this position is titled as senior vice president for financial administration. In some institutions this position is also called vice chancellor for finance, or business manager, or dean of finances, or director of finance.

Student Service Vice President: He or she is the chief of student services referred to in this study with an acronym as CSS, who is responsible for overseeing and giving direction to all student life activities. He or she is usually called the vice president for student service/student affairs/student administration/student life or dean of students, or director of student services/affairs. In some institutions the dean of men and dean of

\footnotetext{
${ }^{57}$ Rahmat Ola Tavallali, "Role and Functions of Presidents and Chancellors of Selected American Public Universities" (Ph.D. dissertation, University of Akron, 1981), 14.

${ }^{58}$ See n. 42.
} 
women do the jobs for this position.

Board of Trustees' Chairperson: The president of a union conference or union mission of Seventh-day Adventists serves as chairperson of the senior college or university board of trustees that governs the union conference's or union mission's educational institution. Normally, a vice president of the General Conference of Seventhday Adventists serves as chair of the General Conference's educational institution. ${ }^{59}$ In this study I have used the acronym, CBT, to refer to the chair of the board of trustees.

Secular Institution: A college or university with no organic religious ties or with only a loose affiliation with a religious denomination or sect.

Seventh-day Adventist Senior Colleges and Universities: Operated by the Seventh-day Adventist Church, senior colleges offer primarily four-year undergraduate programs while the universities offer undergraduate and graduate programs including doctoral programs primarily in the fields of sciences and education. ${ }^{60}$

Adventist Accrediting Association: The accrediting authority for all tertiary and graduate educational programs and institutions, including schools and seminaries, operated in the name of the Seventh-day Adventist Church. It evaluates the quality of the denominational institutions' programs and their implementation of the Seventh-day Adventist philosophy of education in order to foster the unity and mission of the church. ${ }^{61}$

\footnotetext{
${ }^{59}$ Niels-Erik Andreasen, "Servant to the World Church," Focus: The Andrews University Magazine 44, no. 2 (2008): 15. In this article President Andreasen of Andrews University states that "a vice president of the General Conference chairs the university's board of trustees."

${ }^{60}$ Herbert Olivier Shand, "Comprehensive Planning in Seventh-day Adventist Colleges and Universities in the United States" (Ph.D. dissertation, Loma Linda University, 1987), 14.

${ }^{61}$ See n. 6.
} 
The Adventist Accrediting Association ${ }^{62}$ is also broadly known as Accrediting Association of Seventh-day Adventist Schools, Colleges, and Universities.

Administrative Team: The top-level administrators who report directly to the president and with whom they work closely ${ }^{63}$

Higher Education: All post-secondary formal education received in college and university campuses is considered to be higher education. ${ }^{64}$

Philosophy of Education: The Seventh-day Adventist philosophy of education which is Christ-centered and has the unique characteristics of redemptive aim to restore human beings into the image of their Maker. ${ }^{65}$

\section{Summary}

The Education Department of the Seventh-day Adventist world church, known as the General Conference, had circulated to all the SDA higher education institutions a list of subjects for research. One of the topics was the role and function of Seventh-day Adventist college/university presidents. I responded to this request and embarked upon this study. I posed five research questions to achieve the threefold purposes of this study that examined the actual and expected roles of the presidents of the SDA senior colleges and universities around the world, as perceived by the board chairs, presidents, academic vice presidents, student service vice presidents, and finance vice presidents.

\footnotetext{
${ }^{62}$ Seventh-day Adventist Yearbook 2008, 443.

${ }^{63}$ Anna Neumann, "The Thinking Team," The Journal of Higher Education 62, no. 5 (1991): 571.

${ }^{64}$ General Conference Working Policy, 2007-2008 ed., 249.

${ }^{65}$ Ibid., 241.
} 


\section{Organization of the Study}

This dissertation contains five chapters:

Chapter 1 presents the introduction, problem, purpose, importance, need, and assumptions of the study. It states the research questions to be answered and the research hypotheses to be tested; discusses pertinent theoretical concepts; alludes to delimitations and limitations; defines terms, and, finally, provides an overview of the study.

Chapter 2 reviews related literature concerning role and functions of college and university presidents. Since there has not been any research done on perceptions and expectations regarding Seventh-day Adventist senior college and university presidents worldwide, this chapter looks into pertinent literature and nine dissertations on public and other non-Seventh-day Adventist college/university presidency. It also examines some Seventh-day Adventist essays and other publications that have expounded on the concept.

Chapter 3 presents the methodology and the research design. It reviews two related studies and discusses the validity of the instruments used. It examines the subjects involved, the results of the instrument study conducted, the null hypotheses tested, and the collection and treatment of data received from the respondents.

Chapter 4 contains a presentation and analysis of the data for five research questions and three corresponding null hypotheses, and an interpretation of the results.

Finally, chapter 5 summarizes and discusses the findings of the study, draws conclusions, and makes recommendations for future research and practice.

Appendices, bibliography, and a vita complete this dissertation. 


\section{CHAPTER II}

\section{SELECTED REVIEW OF LITERATURE}

\section{Introduction}

There is a considerable amount of literature on the role of the elementary and secondary school principals. Not very many studies have been done concerning the role of college and university presidents. And so far, no studies have been undertaken on the actual role performances versus expected role responsibilities of presidents of Seventhday Adventist senior colleges and universities worldwide.

There is a reflection regarding the operations of a college and university president. One of the reasons is the varying role perceptions and expectations of the president by the various status groups. This chapter discusses the position of college and university president and examines what has been discovered or conjectured about his and her role.

This chapter reviews selected literature on the historical evolution of higher education and college/university presidents' roles, role theory, and related studies on public and private college and university presidency. It looks at studies pertinent to the president's role perceptions and role expectations. It discusses the broad areas of the president's functions, and identifies the Seventh-day Adventist college and university president's duties based on literature and job descriptions; and presents a summary. 


\section{Historical Evolution of Higher Education and College/University President's Roles}

The role of president has changed since the start of the first colleges and universities around the world. This study has included select countries to examine the roles and functions of Seventh-day Adventist colleges and universities. A brief review of a sample of studies suggests the changing roles of presidents in public and private colleges and universities. ${ }^{1}$ The five eras of American higher education, as classified by Cohen, reflect the evolution of varied forms of presidential functions. ${ }^{2}$

In the Colonial Era, 1636-1789, the first nine colleges were founded in the New World, ${ }^{3}$ among which Harvard College in 1636 was the first and Rev. Henry Dunster was the first American college president. The president in a colonial college was a clergyman, who was expected to be a spiritual counselor, or pastor, of the students entrusted to his

\footnotetext{
'Philip G. Altbach, "Past and Future of Asian Universities: Twenty-First Challenges," in Asian Universities: Historical Perspectives and Contemporary Challenges, ed. Philip Altbach and Toru Umazkoshi (Baltimore, MD: Johns Hopkins University Press, 2004), 13-31; N. Jayaram, "Higher Education in India: Massification and Change," in ibid., 85-112; Sungho H. Lee, "Korean Higher Education: History and Future Challenges," in ibid., 145-173; Andrew Gonzalez, "The Philippines: Past, Present, and Future Dimensions of Higher Education," in ibid., 279-290; see also Alberto Amaral, Vincent Lynn Meek, and Ingvild Marheim Larsen, eds., The Higher Education Managerial Revolution? Higher Education Dynamics, 2003, www:springer.com/education/journal/11162 (accessed March 27, 2008). Amaral et al.'s book discusses emergence of managerialism in eleven countries; see also Rudolph $E$. Klimes, ed., Studies in Seventh-day Adventist Higher Educational Administration (Berrien Springs, MI: Andrews University, 1979), 12-17, 25-27; Klimes, ed., Presidents and Boards in Seventh-day Adventist Colleges (ibid.), 2-29, 154-158; Edwin Carlton Walter, "A History of Seventh-day Adventist Higher Education in the United States" (Ph.D. dissertation, University of California, Berkeley, 1966).

${ }^{2}$ Arthur M. Cohen, The Shaping of American Higher Education: Emergence and Growth of the Contemporary System (San Francisco, CA: Jossey-Bass, 1998), 5-291. Cohen classified the American higher education into five eras: (1) The Colonial Era: 1636-1789, (2) The Emergent Nation Era: 1790-1869, (3) The University Transformation Era: 1870-1944, (4) The Mass Higher Education Era: 1945-1975, and (5) The Contemporary or Modern Era: 1976-1999.

${ }^{3}$ Paul Westmeyer, An Analytical History of American Higher Education, $2^{\text {nd }}$ ed. (Springfield, IL: Charles C. Thomas, 1997), 8-9, 18. The first nine colonial colleges are: Harvard (1636), William and Mary (1693), Yale (1701), Princeton (1746), Columbia (1754), University of Pennsylvania (1755), Brown (1764), Rutgers (1766), and Dartmouth (1769).
} 
care. ${ }^{4}$ His major functions were: taught classes, administered discipline, preached at chapel, preached in church on Sunday, kept student records, kept the library, raised funds, and handled the day-to-day business of the college. His titles were master, president, rector, provost, principal, ${ }^{5}$ or chancellor. ${ }^{6}$

The Emergent Nation Era, 1790-1869, was marked by several events, among which opening of the West was notable in that it not only impacted the higher education in the United States but beyond its borders as well. The period gave rise to small colleges, including state colleges, and the beginnings of access for different types of students. New curriculum, new types of faculty, and a new societal context in the wake of an emerging nation brought new challenges to leadership roles. ${ }^{7}$ Other denominational institutions of higher education were founded throughout the first half of the nineteenth century. ${ }^{8}$ In the formative stage of this period the president's responsibilities encompassed the full range of duties, including those of the colonial era. Later, many of these were assigned to such subordinate functionaries as deans, registrar, burser, treasurer, and so on. ${ }^{9}$ 1930), 50, 55.

${ }^{4}$ George P. Schmidt, The Old Time College President (New York, NY: Columbia University Press,

${ }^{5}$ Westmeyer, 19.

${ }^{6}$ Christopher J. Lucas, American Higher Education: $A$ History, $2^{\text {nd }}$ ed. (New York, NY: Palgrave Macmillan, 2006), 124.

${ }^{7}$ Cohen, 56-83.

${ }^{8}$ James A. Patterson, Shining Lights: A History of the Council for Christian Colleges \& Universities (Grand Rapids, MI: Baker Academic, 2001), 17.

${ }^{9}$ Lucas, 120-124. 
During the next three quarters of a century that marks the University Transformation Era, 1870-1944, the research university made its appearance, faculty professionalization took a leap forward, and the role of the state expanded.

Industrialization was triumphant by the beginning of the period..$^{10}$ During these post-Civil War years, the need for a new type of education was felt.. ${ }^{11}$ As a result, the number of new types of students grew markedly, faculty swelled, academic programs multiplied, and the governance displayed an increasing trend toward secularism. Economic and social demands changed the role of president from teacher to administrator, giving him "significant power in his own right."12 Some presidents performed autocratically, demanding that they have a part in all decisions. Others developed hierarchical management systems. Few maintained more than the semblance of a democracy. ${ }^{13}$

The Mass Higher Education Era, 1945-1975, was considered as the golden age for American higher education. Enrollments, institutions, faculty, research, curriculum--all aspects of the system expanded over the earlier eras. The trends in all the areas accelerated. ${ }^{14}$ Increasing reliance on tuition and funding was quite challenging. Complexity in management appeared too great. Most institutions divided their administrative structure into three general functions: academic affairs, student affairs, and

${ }^{10}$ Cohen, 98 .

"Westmeyer, 84.

${ }^{12}$ Frederick Rudolph, The American College President: A History (Athens, GA: University of Georgia, 1991), 167.

${ }^{13}$ Cohen, 97.

${ }^{14}$ Ibid., 152. 
business affairs. Much of president's responsibilities were delegated to his subordinate administrators. ${ }^{15}$ In the 1990s, the president's job was considered more of an art than a profession. ${ }^{16}$

The Modern Era, 1976-1999, brought a turbulent time for the colleges and universities of the United States in particular and presumably other countries in general. The presidents felt uneasy in their leadership roles. The financial crunch of decreasing tuition revenue, increasing costs, demographic changes in typical college-age students, and the country's diverse population had resulted in smaller pools of traditional students who could afford higher education. The president's role has now turned primarily to an administrative role varying from institution to institution. Size of student body, type of institution and its supervisory structure, varieties of programs offered, geographic location, and historical background of institutions, all influence the role of the president. ${ }^{17}$ He has a greater accountability, since the college or university is seen as a business and stakeholders expect deliveries. He is engaged in friend-raising and fund-raising. ${ }^{18}$

During the twenty-first century the role of the president has evolved from paternalistic overseer, to administrator, to fund-raiser over the past more than two hundred years. The challenges of the president's roles in the contemporary era will

${ }^{15}$ Ibid., 245.

${ }^{16}$ Schmidt, quoted in Judith A. Rile, "The Changing Role of the President in Higher Education," 2001, 3, www:newfoundations.com/OrgTheory/Rile721.html (accessed January 14, 2008).

${ }^{17}$ Rile, 3.

${ }^{18}$ L. Wiseman, "The University President: Academic Leadership in an Era of Fundraising and Legislative Affairs," in Managing Institutions of Higher Education into the $21^{\text {st }}$ Century: Issues and Implications, ed. Ronald R. Sims and Serbrenia J. Sims (New York, NY: Greenwood Press, 1991), 5, cited by Rile, 3 . 
surpass those of all earlier eras discussed. The traditional college-age students are being replaced by adults who are enrolling in special adult education programs that deal with issues not normally addressed by typical teenage students. Many earn degrees by taking web-based and web-enhanced courses at satellite locations without having to set foot on college campuses, or do video-conferencing and use other technology without leaving their homes. What role will the president have in these changes? Will the role of the president be like a parent or counselor of the bygone days $?^{19}$ Will there even be a need for a chief executive when there is a provost, who functions as chief operations officer, and also vice presidents delegated with various responsibilities? All these challenges bring new dimensions to the president's role and function in the twenty-first century.

\section{Role Theory}

\section{Role Perception}

One of the early proponents of the concept of role was Ralph Linton. ${ }^{20}$ In 1945, he stated that role presents the sum total of cultural behaviors that are seen associated with a particular position. ${ }^{21}$ Role is a vital part of the position one holds. It is more of a normative cultural pattern or category than an actual behavior. More recently, Hanson attaches his endorsement to this view. ${ }^{22}$ Parsons, on the other hand, maintained that role

\footnotetext{
${ }^{19}$ Rile, 5.

${ }^{20}$ Bruce J. Biddle, Role Theory: Expectations, Identities and Behaviors (New York, NY: Academic Press, 1979), p. ix. 1945), 77.

${ }^{21}$ Ralph Linton, The Cultural Background of Personality (New York, NY: D. Appleton-Century,

${ }^{22}$ E. Mark Hanson, Educational Administration and Organizational Behavior, $5^{\text {th }}$ ed. (Boston, MA: Allyn and Bacon, 2003), 54.
} 
is a functional aspect of a person's participation in a social system. ${ }^{23}$ It is in this aspect that actors are oriented to each other and play a role. According to Lenski, role refers to a position which an individual occupies and to which is attached distinctive behavioral expectations and requirements. ${ }^{24}$ In the light of the above role studies this study deals with the role perceptions of Seventh-day Adventist senior college and university presidents in terms of their actual performances.

\section{Role Expectation}

The concept of role expectation, though prima facie appears to be simple, is wider in its domain of applicability and explanation. Stogdill, Scott, and Jaynes defined role expectation as having two aspects. One is the self-expectations and the other is the expectations by others. The first type refers to an occupant's definition of appropriate behavior for a position which he or she occupies. The second type refers to the expectations by other persons regarding an occupant's behavior in his or her status, expectations which may or may not coincide with one another, or with those of the occupant. $^{25}$ Gross, Mason, and McEachern maintained that role expectations are a set of standards applied to evaluate a person holding elective position or an incumbent. ${ }^{26}$

\footnotetext{
${ }^{23}$ Talcott Parsons, The Social System (Glencoe, IL: Free Press, 1951), 25.

${ }^{24}$ Gerhard Lenski, Human Societies: A Macrolevel Introduction to Sociology (New York, NY: McGraw-Hill, 1970), 39.

${ }^{25}$ Ralph M. Stogdill, Ellis L. Scott, and William E. Jaynes, Leadership and Role Expectations (Columbus, $\mathrm{OH}$ : Bureau of Business Research, College of Commerce and Administration, Ohio State University, 1956), 3.

${ }^{26} \mathrm{Neal}$ Gross, Ward S. Mason, and Alexander W. McEachern, Explorations in Role Analysis: Studies of the School Superintendent Role (New York, NY: John Wiley, 1966), 58.
} 
Owens considered role expectation as expectation that one individual has of the role behavior of another position occupier. ${ }^{27}$ The role expectations of presidents of Seventhday Adventist senior colleges and universities have been considered in the light of the above theories.

\section{Role Conflict}

Parsons propounded that role conflict is an exposure of individuals to such conflicting sets of agreed role expectations that it is not possible to fulfill them. ${ }^{28}$ According to Kahn, role conflict is the simultaneous occurrence of two sets of requirements, compliance of one of which makes it more difficult for the other. ${ }^{29}$ Today, many campuses have chief operations officers who look after the day-to-day operations of the institutions. Additionally, several vice presidents on each campus shoulder responsibilities in their assigned areas. There are chances of role conflicts between presidents and their support administrators. This study looks into the extent of perceptions and expectations of status groups regarding presidents' roles.

\section{Related Studies on Public and Private College/University Presidency}

A review of pertinent literature on the public and private college/university president presents a diverse and interesting confluence of opinions, which were often

\footnotetext{
${ }^{27}$ Robert G. Owens, Organizational Behavior in School (Englewood Cliffs, NJ: Prentice-Hall, 1970), 72.

${ }^{28}$ Parsons, 280.

${ }^{29}$ Robert L. Kahn, Organizational Stress: Studies in Role Conflict and Ambiguity (New York, NY: John Wiley, 1961), 19.
} 
anecdotal recollections of the presidency. In the late 1930s, Twing's volume provided considerable insights into the pressures and problems of the president. He was one of the hypothesizers who viewed the presidency from near and far away, but projected impressionistic feelings about the president. ${ }^{30}$ In the 1940s, Thorpe suggested only vague ideas regarding the actual functions of the president. ${ }^{31}$ A detailed study on administrators is considered more valuable than the anecdotal reports by the presidents. A marked change was noted in the president's functions since the pre-1950s, and the constant change in approaches to the president continued during the tumultuous era of the 1960s. With the passage of time and growth of colleges, the president's responsibilities have increased, while traditional approaches to administration have apparently become less palatable to students, faculty, and board. During the past four decades much attention has been given to educational administration.

In his presidential address to the National Association of State Universities Transactions and Proceedings in 1950, Ila D. Weeks presented his findings regarding the amount of time a college or university president spends, on average, in each of the broad areas of his functions, such as, educational, general administration, financial, physical facilities, and public and alumni relations. Later in 1960, Corson summarized these perceived roles and functions as follows:

Forty percent of their time to financial matters: framing the institution's annual operating budget; presenting it to the trustees and to the legislature; reviewing with advisers the management of invested endowments; and especially appealing to prospective donors.

\footnotetext{
${ }^{30}$ Erwin Twing, The College President (New York, NY: Macmillan, 1939).

${ }^{31}$ A. David Thorpe, College Administration (Boston, MA: Narcisist Press, 1945).
} 
Twenty percent to public and alumni relations: meeting with, and usually addressing, a variety of public, alumni, and parent groups, including state and national educational associations; writing for alumni publications and working with the alumni secretary; and participating in service club or church activities or corporate boards of directors.

Eighteen percent to educational matters: meeting with faculty representatives or otherwise working on matters of educational programming; meeting prospective new faculty members; selecting and promoting faculty members, department heads, and deans; meeting with student groups and student leaders and with staff, studying student problems, including those arising out of the fraternities, and especially out of intercollegiate athletics.

Twelve percent to problems of physical facilities: developing plans for needed classrooms, buildings, laboratories, dormitories, and other facilities with either legislative committees or the donors providing needed funds.

Ten percent to general administration: dealing with university business officers, and meeting with board to consider such problems as the management of dormitories; maintenance of grounds and buildings; procurement of supplies, treatment of clerical, custodial, and other nonacademic employees; or the public problems of the university press. ${ }^{32}$

Mayhew, in 1962, realized that it is not possible for presidents to carry out all the functions. He stated that presidents' roles should be to delegate responsibilities and authorities among their support administrators. ${ }^{33}$ This view has received support since then. More than four decades later, Lawrence, in his 2006 volume, which is a collection of views from 13 presidents and chancellors who presided over 23 American universities from the early 1990s through the shock of $9 / 11$, gleaned the idea of delegating authority, distributing responsibilities, and fostering team work as follows:

Command and control leadership has never been the key to success in the university presidency....

${ }^{32} \mathrm{John}$ J. Corson, Governance of College and Universities (New York, NY: McGraw-Hill, 1960), 59-60; see also Ila D. Weeks, "The University President and the Public," Presidential Address, National Association of State Universities Transactions and Proceedings, 1950, 12-25.

${ }^{33}$ Lewis B. Mayhew, The Smaller Liberal Arts College (Washington, DC: Center for Applied Research in Education, 1962). 
Every leader interviewed gave an important role in their administration to the absolute necessity for teamwork....

Leadership in universities becomes more powerful when it is widely distributed, its goals are universally understood, and its accomplishments become the responsibility of the entire organization. ${ }^{34}$

In the late 1960s, Blackwell classified functions of the president into four major areas: academic affairs, student services, business, and public relations. ${ }^{35}$ According to Simon, in 1967, the president's role is to raise funds; handle budget; set institutional goals; work closely with faculty to create an environment that encourages learning for both students and faculty; and to recruit and maintain a high quality of faculty. ${ }^{36}$ The following year Ingraham and King proposed that the president must function as an internal educational leader, a constant speaker before the alumni, a policy consultant, and a mendicant in his relations to government agencies and foundations. ${ }^{37}$ In 1970, Soshnik maintained that the president's role is planning, organizing, staffing, directing, coordinating, reporting, and budgeting, and that academic and financial functions go hand in hand. ${ }^{38}$ One year later Bolton and Genck stated, though vaguely, that the presidents are expected to provide leadership, set goals, stimulate innovation, and encourage change in

${ }^{34}$ Francis L. Lawrence, Leadership in Higher Education: Views from the Presidency (New Brunswick, NJ: Transaction Publishers, 2006), 444-445. Lawrence interviewed 13 presidents and chancellors of American Ivy League universities and incorporated their views on presidency in this volume.

${ }^{35}$ Thomas Edward Blackwell, College and University Administration (New York, NY: Center for Applied Research in Education, 1996), 14-15.

${ }^{36}$ Herbert A. Simon, "The Job of a College President," Educational Record 68 (1967): 69-70.

${ }^{37}$ Mark H. Ingraham and Francis P. King, The Mirror of Brass: The Compensation and Working Conditions of College and University Administrators (Madison, WI: University of Wisconsin Press, 1968), 256.

\footnotetext{
${ }^{38}$ Joseph Soshnik, "The Campus Presidency," in Perceptions in Public Higher Education, ed. Gene A. Budig (Lincoln, NE: University of Nebraska Press, 1970), 5.
} 
the operation of the university. ${ }^{39}$

\section{Related Studies on Perceptions and Expectations of President's Roles}

In 1960, Nelson's study on the "Role Expectations for Selected College and University Presidents" was predominantly concerned with White, private Eastern colleges and universities in Connecticut, Maine, Massachusetts, New Hampshire, Rhode Island, and Vermont and compared the results to see whether there was any divergence and convergence of the expected roles between the groups. The subjects, comprising of twenty-six college/university presidents and 104 board members, responded to 120 role expectation items on the questionnaire. Nelson paid close attention to personality traits of White presidents in Eastern colleges. His attempt to investigate administrative functions and perceptions of presidents was, clearly, secondary. His sample employed only fouryear college presidents. He tended to study particular personality aspects of the president rather than functions of the presidency. His major findings were that the majority of the presidents and board members held diverging expectations for the role of college and university presidents on: personal qualities, performances, participation, and friendship items. He also found that after gaining several years of experience as board members many of them began to agree with the president's view. ${ }^{40}$

Fern Stout's dissertation in 1962 was "A Comparison of Role Perceptions of

\footnotetext{
${ }^{39}$ Earl C. Bolton and Fredric H. Genck, "Universities and Management," The Journal of Higher Education 13 (1971): 281.

${ }^{40}$ Lawrence O. Nelson, "Role Expectations for Selected College and University Presidents" (Ph.D. dissertation, Michigan State University, 1960), 136.
} 
Selected University Presidents and Their Reference Groups." ${ }^{41}$ His study was conducted to determine: (1) Perceptions which the presidents held about selected behaviors related to the performance of their administrative duties; (2) presidents' perceptions of the expectations held by the department heads, boards of regents, and Boards of Educational Finance with respect to these behaviors; and (3) actual expectations of these same reference groups with respect to these same selected behaviors. ${ }^{42}$ Stout includes higher educational institutions located in New Mexico. This gives a narrow set of conclusions because: there is no means of stratifying such a sample; the perceptions of the president's role may be slanted by peculiarities within the system of higher education in New Mexico; and because the total number of institutions is small, the study could not make any supportable generalizations regarding perceptions of the role of the president. Stout considers his study much of a pilot survey to determine the viability of the topic. ${ }^{43}$

In 1963, Henry Hutchins wrote his dissertation entitled "Role Expectations of Selected College and University Presidents. ${ }^{144}$ He used Black colleges--private, public, and parochial--in Georgia as a basis, and conducted his study to determine role expectations of Black presidents, employing "responses from board members and

${ }^{41}$ Fern D. Stout, "A Comparison of Role Perceptions of Selected University Presidents and Their Reference Groups” (Ph.D. dissertation, University of New Mexico, 1962), 6.

${ }^{42}$ Samuel L. Silas, "Role Perceptions of Presidents in Predominantly Black State and Privately Supported Colleges and Universities as Perceived by Trustees, Presidents, and Deans" (Ph.D. dissertation, Southern Illinois University, 1974), 18.

${ }^{43}$ Arnold Robert Oliver, "The University Presidency as Perceived by Boards of Trustees, Presidents, and Academic Vice Presidents in Selected Colleges and Universities" (Ph.D. dissertation, Southern Illinois University, 1972), 31, 32.

${ }^{44}$ Henry Thomas Hutchins, Jr., "Role Expectations of Selected College and University Presidents" (Ph.D. dissertation, University of Oklahoma, 1963), 158. 
presidents only. ${ }^{145}$ His study was aimed at ascertaining, on demographic information, similarities and dissimilarities of expectations which incumbent presidents and board members held for the role of college or university presidents. ${ }^{46}$ His purpose was "to determine personal characteristics of Black college presidents more so than the role of the president as perceived by board members and academic vice presidents. Hutchins attempts to describe the president rather than deal with the expectations and perceptions of his or her role. ${ }^{n 47}$ His study found out that the majority of the presidents and board members held converging expectations for the role of college/university presidents on: 43 of 56 personal qualities; 31 of 36 performances; 8 of 12 participations; and 10 of 16 friendship items. He also detected that 21 out of 120 role expectation items were capable of producing conflict in role expectations. Hutchins's study was not designed to determine the president's role performance as perceived and expected by himself/herself and board members.

The same year another study, titled, "The Role of the President in American FourYear Liberal Arts Colleges Conducted by the Benedictine Sisters," was conducted by Sister Hausmann. The subjects were composed of seven college presidents. Her findings revealed that although the superior of the religious community operating the college had traditionally held the position of college president, the complexities and demands of modern administration had brought about the trend to separate the office of the religious

\footnotetext{
${ }^{45}$ Silas, 19.

${ }^{46}$ Ibid.

${ }^{47}$ Oliver, 33.
} 
superior from that of the college president. ${ }^{48}$

Massey's study in 1969 dwelt on the complex role of chief executives in 294 universities and colleges which were affiliated with the Southern Association of Colleges and Schools. ${ }^{49}$ He compared the roles depicted in the literature, and the perception of the roles by the chief executives as reflected in their responses to the questionnaire. The findings revealed differences and similarities that existed between the data gathered and the information obtained in the review of literature. Among the major findings were: The role of the president varied with the control of his institution, whether private or public. The incumbent believed that he should assume the role of a strong leader, take the initiative, and lead the members of the governing board; the chief executive had essential fund-raising and public relations responsibilities and was more of a business executive than intellectual leader; and administrative activities took up about 32 percent of the chief executive's working time.

In 1973, Trimble embarked on a study to investigate the priorities of roles of presidents at twelve diverse colleges and universities in Massachusetts. The six subjects at each institution were comprised of the president, a board or regent officer, two elected faculty leaders, and two elected student leaders, making a total population of 72 for 12 institutions. Responses received from the questionnaire and interview yielded results that

\footnotetext{
${ }^{48}$ Sister M. Daniel Hausmann, "The Role of the President in American Four-Year Liberal Arts Colleges Conducted by the Benedictine Sisters" (Ph.D. dissertation, The Catholic University of America, 1963), Abstract.

${ }^{49}$ James William Massey, "The Role of the Chief Executive in Universities and Senior Colleges Which Are Members of the Southern Association of Colleges and Schools" (Ph.D. dissertation, University of Mississippi, 1969), Abstract.
} 
showed presidents functioned primarily in managerial and supporting roles. ${ }^{50}$

Oliver's study in 1975 investigated the role of presidential administrative functions relative to expectations, perceptions, and modes of operation as perceived by the presidents, academic vice presidents, and boards of trustees. ${ }^{51}$ The findings of the Oliver study revealed areas of congruence and non-congruence in most combinations of reference groups, such as, agreement relative to given variables was found: (1) to exist among the governing board members and the presidents but to be opposed by the academic vice presidents; (2) to exist among the presidents and academic vice presidents but to be opposed by the board members; (3) to exist among the presidents but to be opposed by the academic vice presidents and board of trustees; (4) to exist among the academic vice presidents but to be opposed by the presidents and members of boards. ${ }^{52}$

Some of the major specific agreements and disagreements among the status groups on the matter of presidency found in the Oliver study were: (1) that the majority of the board members held similar views regarding the functions of the presidents; (2) that there was general consensus among the presidents as to how they interpret the president's job; (3) that the academic vice presidents showed more disagreement than the presidents or board members as to how they view the presidential function; (4) that there was no lack of consensus among the academic vice presidents regarding their views on the functions of the presidents; (5) that significant disagreement existed among the academic

\footnotetext{
${ }^{50}$ Jerry Max Trimble, "Priorities of College and University Presidents" (Ph.D. dissertation, Boston College, 1973), Abstract.

${ }^{51}$ Oliver, 6-8.

${ }^{52}$ Ibid., 49-55.
} 
vice presidents, presidents, and board members on perceptions of performances of the president's role; and (6) that there was about as much agreement with the academic vice presidents as board members. ${ }^{53}$

From the above findings, Oliver concluded that the presidents saw much greater flexibility in the university presidential administration than did the academic vice presidents and members of the governing board. ${ }^{54}$ His study, of course, did not compare between the actual and expected roles of the president.

The difference between the previous studies and the Oliver study is that the former, namely, Nelson, Stout, and Hutchins studies, described the personality traits of the president, while the latter attempted to deal with the role expectations and perceptions of the president. ${ }^{55}$ The former studies gave a narrow set of conclusions and could not make any generalization regarding the role perception of the president. In fact, no study has been done so far on Seventh-day Adventist senior college and university presidents' actual versus expected roles.

The Silas study was concerned with the "Role Expectations of Black Presidents in Predominantly Black State and Privately Supported Colleges and Universities as Perceived by Boards of Trustees, Presidents, and Deans," as compared to Oliver's study that included the presidents, academic vice presidents, and boards of trustees from forty of the largest Caucasian public institutions of university status only belonging to the

\footnotetext{
${ }^{53}$ Ibid., 93-97.

${ }^{54}$ Ibid., 23-24.

${ }^{55}$ Ibid., 33.
} 
American Association of State Colleges and Universities. The Silas study sought to identify areas in which misunderstanding and disagreement existed, communication was interrupted, and produced data for possible corrective action in the predominantly Black colleges administered by administration. Silas's dissertation secured information that will help the president, the dean, and the governing board to understand more clearly what is being asked of the president. Another major difference between the Oliver's and the Silas's studies lies in the area of presidential origin, qualifications, and administrative philosophy. That is, the Silas study investigates whether the president was selected from the college in which he or she was employed or from which he or she graduated, whether he or she had prior administrative experience on the job, or whether he or she had studied an administration course in the formal classroom. Oliver's study was not designed to investigate into these areas.

The seven areas that Silas covered are: (1) demographic data, (2) perceptions of the role and philosophy of the president, (3) necessary background for a president, (4) perceptions of specific actions of a president, (5) presidential interaction, (6) forced ratings by presidents regarding their functions in terms of importance, preference, and success, and (7) open-ended questions relating to presidential administrative functions and to the present and projected status of higher education as it affects college and university presidents. Silas's research investigates personality traits, administrative functions, and perceptions of Black college and university presidents of predominantly Black public and private four-year institutions. ${ }^{56}$

${ }^{56}$ Silas, 17-25. 
Kim's study, titled, "A Study of Role Expectations of Presidents in Seventh-day Adventist Colleges," was to ascertain the role expectations of presidents as perceived by eight presidents, 212 trustees, and 344 faculty members who had responded to 75 questions classified under eight broad areas of functions, such as, administration, board relations, faculty relations, finance, physical facilities, student personnel, public and alumni relations, and spiritual affairs. The purpose of the study was to determine whether or not the expectations of these groups were conflicting or in agreement. Kim's findings were summarized as follows: 5 items of the 75 items revealed conflict between the presidents and board members; 5 items of the 72 items showed conflict between the presidents and faculty members; and 29 items out of 75 items revealed conflict between the board members and faculty members. ${ }^{57}$

Although the titles and procedures of the above studies might appear to be somewhat similar to one another, their intents and purposes were different from each other. Therefore, the findings and conclusions were vastly different in each investigation. However, each of the studies was unique and had adequately fulfilled their purposes. All of the nine dissertations mentioned in this section are quite different from the present study. First, the sample size used in this study must allow some degree of universality, since it included 28 senior colleges and universities from the various geographic divisions of the world. Second, it covered only the Seventh-day Adventist Christian institutions of higher learning. Third, this study has five status groups, such as,

\footnotetext{
${ }^{57}$ Chong Wha Kim, "A Study of Role Expectations of Presidents in Seventh-day Adventist Colleges" (Ph.D. dissertation, University of Nebraska, 1975), 63-64. Kim's study did not include colleges outside of the United States.
} 
board chairs, presidents, academic vice presidents, student service vice presidents, and finance vice presidents. Fourth, it attempted to cover the actual and expected roles of presidents of Seventh-day Adventist senior colleges and universities based on the unique mission. None of the research studies reviewed in this section aimed at finding similarities and dissimilarities existing between the actual role performances and expected role responsibilities of presidents.

The role of president has changed markedly over the last quarter of a century and so has the approach to the study of the president's office. Today, the chief executive of a senior college or university must deal with a variety of problems, such as, buildings, money, organization, legal provisions, educational programs, and human beings. ${ }^{58}$

People within and outside the college and university structure have varying and often conflicting expectations of the president and how he or she acts. The presidents themselves also have their own concepts of what their roles and functions ought to be. Their self-concept might lead them to pursue one line of actions or behaviors. On the other hand, they might feel obligated to fulfill the expectations of other individuals, if they want to be successful in their presidential functions. Stogdill stated,

True leadership role is determined, as are other roles, although carrying varying degrees of flexibility, by the expectations of the leader and of the other group members. Role behavior, what the leader actually does, therefore becomes the resultant of what he and others think of behaviors that are required, prohibited, and allowed..$^{59}$

In 1955, the findings of Getzels and Guba showed that teachers determined that

\footnotetext{
${ }^{58}$ Ronald F. Campbell, John E. Corbally, and John B. Ramsayer, Introduction to Administration (Boston, MA: Allyn and Bacon, 1953), 17.

${ }^{59}$ Stogdill, quoted in Oliver, 22.
} 
certain aspects of a teacher's self-perceptions and society's expectations did not match properly. They attempted to define the role conflict. ${ }^{60}$ Their study tends to suggest the same concept of college/university presidents' role perceptions and role expectations.

There is a firm theoretical foundation for a study of university or college presidents from the point of view of governing board and college or university administrative officers to determine perceptions and expectations of the president's role. Through the years, different theoretical models have been developed. It does seem apparent that the study by Getzels, Lipham, and Campbell is a culmination of all the research regarding role expectation and perception. They do not consider their volume to be definitive, for it, too, is much the same as many of the earlier works. ${ }^{61}$

\section{Broad Areas of President's Functions}

Vineyard states that the role of president is often operationally defined in practice in terms of expectations or demands, as significant as the job description in the policy and procedures manual may be. ${ }^{62}$ The president may be held responsible for everything which transpires within the institution. The president is responsible for the participation of his or her campus in overall planning, resource allocation, and evaluation operations. ${ }^{63}$ The president's role varies with the size and type of his or her college or university, whether it

\footnotetext{
${ }^{60} \mathrm{Jacob}$ W. Getzels and E. G. Guba, "The Structure of Roles and Role Conflict in a Teaching Situation," Journal of Educational Psychology 24 (1955): 30-40.

${ }^{61}$ Jacob Getzels, James Lipham, and Ronald F. Campbell, Educational Administration as a Social Process (New York, NY: Harper and Row, 1968), quoted in Oliver, 26.

${ }^{62}$ Edwin E. Vineyard, The Pragmatic Presidency (Boston, MA: Anker Publishing, 1993), 10.

${ }^{63}$ Lita Allen Clark, "Role Expectations for the Chief Executive Officer of a University System" (Ph.D. dissertation, University of Alabama, 1977), 47.
} 
be "private" or "public."164 Corson indicates that as the institution grows in enrollment, and hence in faculty, in facilities, and in budget, the president is ejected from the areas of the institution's central concerns--the educational program, the faculty, and the students-by the demands that are made on his or her time by other activities. ${ }^{65}$ Almost all experts view the functions and the role of the college and university president as follows:

1. Educational Programs-Related Functions

2. Student-Related Functions

3. Public- and Alumni-Related Functions

4. Physical Facilities-Related Functions

5. Finance- and Fund-Raising-Related Functions

6. Faculty- and Staff-Related Functions

In addition to the above functions, there is one other category of functions that presidents perform. It is the Spiritual Life-Related Function. The denominational colleges and universities have these functions.

\section{Educational Program-Related Functions}

The university and college president is an educator ${ }^{66}$ His or her primary role is "educational leadership." ${ }^{67}$ Vineyard says,

\footnotetext{
${ }^{64}$ Harold W. Dodds, Academic President: Educator or Caretaker? With the collaboration of Felix C. Robb and E. Robb Taylor (New York, NY: McGraw Hill, 1962), 20.

${ }^{65}$ Corson, 61; see also Charles E. Bouchard, Susan Thistlethwaite, and Timothy Weber, "The President's Role as Academic Leader," in A Handbook for Seminary Presidents, ed. G. Douglas Lewis and Lovett H. Weems, Jr. (Grand Rapids, MI: William B. Eerdmans Publishing Co., 2006), 73-84.

${ }^{66}$ Neely Dixon McCarter, The President as Educator (Atlanta, GA: Scholars Press, 1996), 43-60.

${ }^{67}$ Corson, 62.
} 
Although the college is filled with academic specialists, the president must not forget that he, too, is an academician by education and by experiential background. He must not become so preoccupied by other distractions in management that he neglects this most important sphere of operations within the institution. ${ }^{68}$

In the 1970s, the La Sierra Campus of Loma Linda University, together with four former Seventh-day Adventist college and university presidents, organized an updated study determining the amount of time the college/university president spends on various roles. This study gave credit to I. D. Weeks, C. Kerr, J. J. Corson, J. D. Millett, J. W. Nason, M. D. Cohen, and J. G. March for their initial studies on the subject. ${ }^{69}$ A summary of findings based on analyses showed that the chief executives of modern universities, on the average, devote $18-20$ percent of their time to educational matters, such as educational programming, academic master-planning, meeting with faculty representatives, deans, department heads, etc.; meeting with student groups and student leaders, and handling staff with a wide variety of student-related matters and problems. The educationists picture the president not spending more than one-fifth of his or her time working on educational matters and keeping in touch with the school's faculty.

His or her role is not as large as might be supposed and tends to be indirect, especially if the institution has a vice-president responsible for academic affairs. The

${ }^{68}$ Vineyard, 90.

${ }^{69}$ I. D. Weeks, "The University President and the Publics," Presidential Address, National Association of State Universities Transactions and Proceedings (1950), 12-25 (This was a pioneering study on the subject); see also C. Kerr, The Uses of the University (Cambridge, MA: Harvard University Press, 1964); J. J. Corson, The Governance of Colleges and Universities, rev. ed. (New York, NY: McGraw-Hill, 1975); J. D. Millett, The Multiple Roles of the College and University President (Washington, DC: American Council on Education, 1976); J. W. Nason, Presidential Assessment: A Challenge to College and University Leadership (Washington, DC: Association of Governing Boards of Universities and Colleges, 1980); M. D. Cohen and J. G. March, Leadership and Ambiguity: The American College President (New York, NY: McGraw-Hill, 1974). 
president is seldom the originator of any new idea pertaining to educational program, nor is it often practical for him or her to attend any faculty discussions on innovative educational proposals. Yet, the president should be knowledgeable in all educational programming, since he or she does exercise a decisive influence over the character of the institution's educational offerings through his control and use of the budget. ${ }^{70}$

The president himself must be the number one guardian of academic standards."71 He should take the lead in matters relative to courses, curricula, and educational programs. Customarily, the president presides over the university senate, or college faculty meeting, and serves as an ex-officio member of its principal committee. ${ }^{72}$ Today, most modern university and college presidents believe that they should be able to frame the educational character of their institutions. ${ }^{73}$

\section{Student-Related Functions}

Literature indicates that in all the roles of student affairs, the presidency needs to look at desirable goals and outcomes. According to Flawn, ${ }^{74}$ the mission of the university

\footnotetext{
${ }^{70}$ Loma Linda University, La Sierra Campus, "The Role of the President in Contemporary American Universities: A Summary of Finding With Brief Recommendations" (Riverside, CA: Administrative Affairs Committee, 197-?), 1-6, James White Library, Andrews University, Berrien Springs, MI. The study gives credit to I. D. Weeks, C. Kerr, J. J. Corson, J. D. Millet, J. W. Nason, M. D. Cohen, J. G. March and four former presidents of SDA colleges and universities for their studies on the role of the president in contemporary American universities. The findings and recommendations of the Loma Linda University study in the 1970s had to do with the amount of time the presidents were found to have spent in various educational program-related functions and the need for degrees of emphasis on these activities.

${ }^{71}$ Vineyard, 90.

${ }^{72}$ Corson, 62.

${ }^{73}$ Tavallali, 24.

${ }^{74}$ Peter T. Flawn, A Primer for University Presidents: Managing the Modern University (Austin, TX: University of Texas Press, 1990), 90.
} 
is to develop the human resources of society. The students are the mission of the institution. ${ }^{75}$ The university is attempting to develop the students--intellectually. Corson maintains that a frequent complaint is that the president has too little time to devote to student affairs. ${ }^{76}$ The students are the primary reason the university or college exists and "no president can truly be considered a success, if he or she does not serve students well. ${ }^{177}$ Because of other matters that consume a president's time, decisions on matters arising out of student's academic affairs remain in the faculty, while decisions on student's non-academic affairs are made by a students' dean in the larger institutions. But the activities of the dean of students are the ultimate responsibility of the president. ${ }^{78}$ The president then has a responsibility in overseeing the student affairs, although a students' dean or a vice president of student life is there to do this job.

Dodds feels that the president has to take the initiative in making contact with the representative students. He should consider the students' problems and not ignore them. Despite the pressures of management, a good many presidents do find time to involve themselves in student affairs. ${ }^{79}$

While writing on the importance of the college's mission statement that gives rationale for the campus, Meacham stated that it is a declaration of responsibilities toward

\footnotetext{
${ }^{75}$ David McAllister-Wilson, Craig Williford, and David Neelands, "The President's Role in Enrollment Management and Student Issues," in Lewis and Weems, Jr., 141.

${ }^{76}$ Corson, 64.

${ }^{77}$ James L. Fisher and James V. Koch, Presidential Leadership: Making a Difference (Phoenix, AZ: American Council on Education and Oryx Press, 1996), 157.

${ }^{78}$ Corson, 64.

${ }^{79}$ Dodds, 55.
} 
students. He surveyed more than three hundred campuses to identify the student learning goals. ${ }^{80}$ The Education Department of the Seventh-day Adventist world church has suggested that the students be one of the major focuses of the university administration. ${ }^{81}$

\section{Finance- and Fund-Raising-Related Functions}

According to Adams and Kirst, anything that has to do with the welfare of students is subject to accountability. Since finance plays a vital role in operating a college/university, the president is accountable to the board for its financial operation. ${ }^{82}$

In her article, Paolitto presents a number of presidents' opinions regarding finance and fund-raising for college and university ${ }^{83}$ Former Harvard President Derek Bok defines the achievement or failure of a college president by "how much money you raise, not what you raise the money for." John Kenneth Galbraith, America's most famous economist, teacher, and diplomat, notes that the primary function of a college president is to raise money for institutions.

The American Council on Education's National Presidents' Study for the year 2000 ranked fund-raising as the primary use of time by the presidents at all private and

\footnotetext{
${ }^{80}$ Jack Meacham, "What's the Use of a Mission Statement?" ACADEME: Bulletin of the American Association of University Professors 94, no. 1 (2008): 21-24.

${ }^{81}$ See Appendix D, sec. 6.

${ }^{82}$ Jacob E. Adams and Michael W. Kirst, "New Demands and Concepts for Educational Accountability: Striving for Results in an Era of Excellence," in Handbook of Research on Educational Administration, ed. Joseph Murphy and Karen Seashore Louis, $2^{\text {nd }}$ ed. (San Francisco, CA: Jossey-Bass, 1999), 465; see also Kenneth Leithwood and Lorna Earl, "Educational Accountability Effects: An International Perspective," Peabody Journal of Education 75, no. 4 (2000): 6-7.

${ }^{83}$ Julia Paolitto, "President of Yale, or CEO of Yale, Inc.?" www.yaleherald.com/archive/xxxi/2001.04.20/features/front.html (accessed April 2, 2008).
} 
research universities and noted that less than half of all college presidents spend a considerable amount of time on community relations and academic programs. ${ }^{84}$ Reverend Theodore Hesburgh, president emeritus of University of Notre Dame and first educator to receive the Congressional Gold Medal for distinguished service to the nation, accused college presidents of spending too much time raising money, of mistakenly treating their potential moral authority as merely a managerial position, and of remaining "simply too busy to speak out on issues beyond the immediate concerns of their institutions. ${ }^{985}$

In the 1970s, Loma Linda University coordinated a study that included opinions of educational authorities representing public and private colleges, including Seventh-day Adventist institutions. According to this report, the president's role in financial matters appeared strong at all times. The functions include: framing the budget, presenting it to the board, reviewing with advisers the management of invested endowments, and seeking and appealing to prospective donors. While the president may delegate much responsibility to deans, provost, finance vice president, or department heads in the budget-making process, he or she has a responsibility for assembling the various components making up the annual budget and presenting it for approval to the board. ${ }^{86}$ In church-related and private institutions, the president must plan and lead the perennial or continual campaign for financial support and contributions from the church, alumni,

\footnotetext{
${ }^{84}$ Paolitto, 3.
}

${ }^{85}$ Theodore Hesburgh, "Where Are College Presidents' Voices on Important Public Issues?" The Chronicle of Higher Education, February 2, 2001, quoted in Paolitto, 2-3.

${ }^{86}$ Anthony Ruger, John Canary, and Steven Land, "The President's Role in Financial Management," in Lewis and Weems, Jr., 92. 
corporations and foundations, and the public at large. Given the precarious financial situation of most church-related institutions--a condition which is not likely to improve in the years ahead--the quest for funds and the task of balancing the budget are and will remain the most time-consuming, worry-causing, and energy-taxing factors facing a college/university president. ${ }^{87}$

Speaking from his personal experience in developing an annual-giving program at Fitchburg State College that resulted in a deeper river of unrestricted gifts, Drozdowski said that a fund-raising drive will be successful when the donors believe that their donations are being spent on students, faculty members, programs, and facilities. ${ }^{88}$

\section{Public- and Alumni-Related Functions}

According to Prater and Nudd, the president's relations with off-campus people are as important as those he or she has with his or her on-campus associates. In general, they involve the college's relationships with its alumni and its community, and are frequently referred to as "public relations." ${ }^{89}$

All colleges have some plan of interpreting the purposes and mission of their program to the public. Although public relations is everybody's responsibility in the college or university--administration, faculty, trustees, students, and non-academic

\footnotetext{
${ }^{87}$ Loma Linda University Study, 4-5.

${ }^{88}$ Mark J. Drozdowski, "No Strings Attached," The Chronicle of Higher Education 54, no. 21 (February 1, 2008): C 3.

${ }^{89}$ Ralph Prater, The College President (Washington, DC: Center for Applied Research in Education, 1963), 44; see also Garrett W. Nudd, "Practical Public Relations: Making Your School Stand Out Despite Limited Financial Resources," The Journal of Adventist Education 64, no. 1 (2001): 9-13.
} 
personnel--the president is the key person. Any neglect of this responsibility by the president will affect every aspect of the public relations program..$^{90}$

Stoke states that every enterprise which involves a substantial number of people is affected with a public interest. Since every responsibility for the success of the college eventually comes to rest upon the president, he or she finds himself or herself inextricably a public relations officer. This is true whether it is a large university or a small college. ${ }^{91}$

Law states that the president's role as liaison between the institution and the general public (or constituency) needs special consideration. It is his or her function to explicate the actions of his or her college or university to the society which is both its ultimate benefactor and beneficiary. ${ }^{92}$ Corson sees the president's role in public relations as that of a speaker who should devote a great proportion of his or her time to the making of speeches before selected groups, such as the press, public, educators, and alumni. ${ }^{93}$

According to Donovan and Wild, the presidents, whether they like the idea or not, are the chief public relations officers of their institutions. Their success depends very much on how effective they are in dealing with the several groups, such as alumni, community, churches, and constituency, that make up the public. No institution of higher education can live unto itself. It belongs to the public and the public is entitled to know

\footnotetext{
${ }^{90}$ Robert Cooley, Christa Klein, and Lous Weeks, "The President's Role in Governance," in Lewis and Weems, Jr., 38. $90-91$.

${ }^{91}$ Harold W. Stoke, The American College President (New York, NY: Harper \& Brothers, 1959),

${ }^{92}$ Glen Charles Law, The Urgency of New Leadership in Higher Education (Stamford, CT: PressTige Publishing, 1962), 95, quoted in Tavallali, 26.

${ }^{93}$ Corson, 67.
} 
about its program..$^{94}$

The summary of findings of the Loma Linda University's study conducted in 1979 shows that $20-25$ percent of the president's time is devoted to public and alumni relations activities. ${ }^{95}$ Successful public relations boils down to effective communication that makes friends. ${ }^{96}$ Every friend-making activity should be translated directly into fundraising in terms of cash or kind. ${ }^{97}$

Almost all institutions have alumni organizations. Most colleges are keeping in close contact with their graduates, who take active roles in campus affairs.

\section{Faculty-Related Functions}

Fisher and Koch stated that faculty members are the body and the heart of a college or university, for they are the ones who are the most measurable test of a president's leadership, influence, and mission. Faculty members must be autonomous in their disciplines, respected in their governance role, and gently reminded from time to time that final authority is the president. The president must lead, remind, and inspire the faculty beyond themselves. He should stay in touch with the faculty through informal and formal meetings, periodic campus tours, classroom visits, frequent and visible attendance

\footnotetext{
${ }^{94}$ H. L. Donovan and R. W. Wild, "The President and the Public," Peabody Journal of Education 26, no. 5 (2000): 258-263. At the time of writing this article, Donovan was president and Wild was public relations director of University of Kentucky.

${ }^{95}$ Loma Linda University, 1.

${ }^{96}$ Garrett W. Nudd, "Practical Public Relations: Making Your School Stand Out Despite Limited Financial Resources," The Journal of Adventist Education 64, no. 1 (2001): 9; see also Lewis and Weems, Jr., 155-164.

${ }^{97}$ Fisher and Koch, 215.
} 
at campus functions, and attendance at departmental assemblies and socials. ${ }^{98}$

Expert teachers could be recruited, if need be, from places outside of areas where institutions are located..$^{99}$ Policies must also be devised to attract and retain them. ${ }^{100}$ Cornette, president of West Texas State College, Canyon, Texas, stated that the president's role should be to see that faculty salaries receive priority over plant development. ${ }^{101}$ The president reviews all hiring, performance evaluations, salary increases, promotions in light of available resources and overall staffing needs. ${ }^{102}$

The president should make sure that the faculty, as well as students, enjoys the academic freedom. The current international crisis poses serious academic problems. Akker indicated that in many parts of the world there is no academic freedom in the educational institutions. The United Nations Human Rights Commissioner, Marty Robinson, stated that the universities and colleges have a vital role to play in the development of human rights. ${ }^{103}$ According to Zwemer ${ }^{104}$ the teachers and students must always remain free to inquire, to study and to evaluate, and to gain new maturity and

${ }^{98} \mathrm{Ibid}$.

${ }^{99}$ Del Stover, "Teacher Recruiting Especially Challenging for Urban School Boards," The Education Digest 73, no. 3 (2007): 29.

${ }^{100}$ Audrey Amrein-Beardsley, "Recruiting Expert Teachers into Hard-to-Staff Schools," The Education Digest 73, no. 4 (2007): 41; see also Lewis and Weems, Jr., 23.

${ }^{101}$ James P. Cornette, "The President and His Faculty," Peabody Journal of Education 26, no. 5 (1949): 264-267.

${ }^{102}$ Lewis and Weems, Jr., 28.

${ }^{103}$ John Akker, "Protecting Academic Freedom Worldwide," ACADEME 88, no. 3 (2002): 44-45.

${ }^{104}$ Tom Zwemer, "Candor and Civility: A Post-9/11 Review of Academic Freedom," Liberty: A Magazine for Religious Freedom 103, no. 2 (2008): 5. 
understanding; otherwise our civilization will stagnate and die.

Seventh-day Adventists subscribe to the principles of academic freedom generally held important in higher education. ${ }^{105}$ Freedoms are never absolute and imply commensurate responsibilities. It is the duty of the president to "protect the scholar." One clear limitation of academic freedom in SDA institutions which the president must guard against is that faculty do not teach anything contrary to the church's fundamental beliefs. ${ }^{106}$ The president's faculty-related functions, according to the college and university faculty manuals I have looked into, are largely delegated to academic vice presidents. However, it is understood that the presidents have the ultimate responsibility in overseeing the faculty interests.

\section{Physical Facilities-Related Functions}

In the literature there is little information on physical facilities planning for higher education. The procedure described is often quite superficial. The physical facilities of a college/university embrace many aspects. They include the grounds, buildings, furniture, and equipment. They also include transportation, parking, energy, utility, dining services, library, labs, communication services, central storage and warehouse spaces, gymnasium, athletic facilities, and all other facilities needed to operate an institution.

${ }^{105}$ Andrews University, "General Conference Statement on Academic Freedom in Seventh-day Adventist Institutions of Higher Education," Andrews University Working Policy, Section 2, 2:801, www.andrews.edu/faculty/documents/index.html (accessed May 20, 2008).

${ }^{106}$ Bert B. Beach, "Adventists and Higher Education," Adventist Review: The General Paper of the Seventh-day Adventist Church 166, no. 9 (1989): 18-24. In October 1984 the Annual Council of the General Conference Executive Committee of the Seventh-day Adventist World Church upheld the academic freedom in SDA higher education. In 1987 a statement was developed as an official position paper on the academic freedom. The 1987 Annual Council Actions, Nos. 17-19, of the General Conference Executive Committee of the Seventh-day Adventist world church, contains this position paper on academic freedom. 
Emeritus President Charles Vest of Massachusetts Institute of Technology, who was involved in MIT's physical facilities development, stated that great universities evolve intellectually, socially, and physically roughly 50 percent by planning and 50 percent by serendipity. A visionary president hires the most suitable chief facilities officer to look after campus facilities development. ${ }^{107}$ The college or university president may hire an architect or a builder to do the physical facilities planning. He may even assign a faculty to oversee the facilities-related functions. ${ }^{108}$ But it is the president who is ultimately responsible in initiating the long-range facilities planning, and to oversee its smooth implementation. ${ }^{109}$

According to Roscoe, beautiful, orderly, and inspiring campuses seem to attract good students and faculty and make alumni proud and generous. Institutional executives need to create campus environments of high quality so that site-based higher education will inspire the pursuits of the aspirers. ${ }^{110}$ The Churchillian phrase, "we make our buildings and then our buildings make us," 111 can be rightly applied to college presidents' physical facilities-related functions. The faculty handbooks received from the colleges and universities around the world indicate that the physical facilities planning and

\footnotetext{
${ }^{107}$ William J. Mitchell, Imaging MIT: Designing a Campus for the Twenty-First Century (Cambridge, MA: MIT Press, 2007), 124-128.

${ }^{108}$ Basil Castaldi, Educational Facilities: Planning, Modernization, and Management (Boston, MA: Allyn and Bacon, 1994), 316-336.

${ }^{109}$ Ward Ewing, Thomas Graves, and Robert Landrebe, "The President's Role in Managing Facilities," in Lewis and Weems, Jr., 116-117.

${ }^{110}$ Lewis Roscoe, Planning the Campus: Making No Little Plans for Colleges and Universities (Amherst, MA: L \& A Publications, 2000), p. i, 201.

${ }^{111}$ Mitchell, 120.
} 
administration ultimately fall under the chief executive. ${ }^{112}$

Boughman indicated that the educational programs cannot be effectively launched unless adequate physical facilities are available on campus. ${ }^{113}$ Sample case studies reveal that some colleges and universities did physical facilities upgrading and modernizing. As a result, these institutions' deliveries of programs have improved. ${ }^{114}$

\section{Spiritual Life-Related Functions}

The common notion is that spiritual functions have no place in secular, nonreligious institutions. However, most recently Rosenblith and Bailey expressed their optimistic views regarding religious studies in public education. They maintained that religious education will develop knowledge and understanding about various religious traditions, foster greatest respect for the religious other, and think through the existential concerns. ${ }^{115}$ Fite stated that religion and education should work as a team in equipping persons for this world. There can be no vital education without religion. Education and religion are so inherently related that man cannot come to his best with either alone. ${ }^{116}$

${ }^{112}$ Faculty handbooks received from colleges and universities indicate that the president is ultimately responsible for physical facilities planning of the campus.

${ }^{113}$ Larry W. Boughman, "Guidelines for Campus Beautification with Special Reference to SeventhDay Adventist Academies and Colleges in North America" (Ph.D. dissertation, Andrews University, 1991), $1-3$.

${ }^{114}$ Patrick Joseph Keating, "Models for Campus Master Planning and Facility Development: A Comprehensive Case Study Analysis of Four Private Research Universities" (Ph.D. dissertation, Michigan State University, 1988), 62-166.

${ }^{115}$ Suzanne Rosenblith and Bea Bailey, "Comprehensive Religious Studies in Public Education: Educating for a Religiously Literate Society," Educational Studies 42, no. 2 (2007): 93-109. (1949): 268-271. 
The religious objective is considered to be the most important dimension of the Seventh-day Adventist education, because it distinguishes the institution's mission, philosophy, purpose, aims, objectives, and goals from any other secular institution. The Seventh-day Adventist colleges and universities seek to "develop Christian character motivated by love and controlled by steadfast principle, thus preparing the individual for self-government and self-discipline."117

The education and leadership provided in the SDA educational centers should recognize the spiritual journey of the students as they seek meaning for their lives. It is the president's responsibility to see that the college/university family, especially student body, has opportunities to expose themselves to spiritual enrichment so that they can be prepared for a wider service. Ellen White wrote,

If the influence in our college is what it should be, the youth who are educated there will be enabled to discern God and glorify Him in all His work; and while engaged in cultivating the faculties which God has given them they will be preparing to render Him more efficient service. The intellect, sanctified, will unlock the treasures of God's word and gather its precious gems to present other minds and lead them also to search for the deep things of God. ${ }^{118}$

According to Brown, the president is responsible "to promote the religious life of students, faculty, and staff."119 The faculty handbooks received from the Seventh-day Adventist, Catholic, and Protestant colleges and universities clearly specify the spiritual life-related functions of the presidents.

${ }^{117}$ General Conference Working Policy, 2007-2008 ed., 241.

${ }^{118}$ Ellen G. White, Testimonies for the Church, vol. 4 (Boise, ID: Pacific Press, 1948), 422. Ellen White, one of the founders of the Seventh-day Adventist world church, was an inspired writer, who authored a number of books on a variety of subjects, including education.

${ }^{119}$ Brown, sec. 2 J, 66. 


\section{Seventh-day Adventist College/University \\ President's Roles and Functions}

Maphosa states that the basic role and functions of the chief executives of the

Seventh-day Adventist institutions of higher learning are no different from those of other non-Adventist executives. The differences are found in the underlying values, world view, and philosophies that are brought to the workplace by the chief executives. ${ }^{120}$

According to Brown, some of the major functions of Seventh-day Adventist college/university presidents are as follows: ${ }^{121}$

1. Serve as secretary of board of directors and of executive board, and administer their policies and regulations, ensuring the implementation of the same.

2. Preside over faculty meetings and implement policies and regulations of faculty.

3. Promote amity and unity of purpose among all members of teaching and administrative staff, board of directors, constituency, alumni, and student body.

4. Make recommendations to the board on:

--Appointment, compensation, assignment of rank, continuous appointment, promotion, graduate study, leave of absence, and severance of faculty.

--Appointment, compensation, and severance of part-time instructors and other major non-faculty employees.

5. Estimate budget needs in collaboration with administrative officers and present college budget to board.

6. Present to the board, the accrediting agencies, the constituency, and appropriate educational bodies the essential reports concerning the college.

\footnotetext{
${ }^{120}$ Norman Maphosa, "Critical Skills for Chief Executives in Adventist Institutions of Higher Learning," in Christ in the Classroom: Adventist Approaches to the Integration of Faith and Learning, ed. Humbarto M. Rasi (Silver Spring, MD: Institute for Christian Teaching, 1994), 12:144.

${ }^{121}$ Brown, 65-66. Walton J. Brown served at the world headquarters of the Seventh-day Adventist Church's Education Department when he compiled the list of the president's duties.
} 
7. Present to the board the needs of the institution and direct in the preparation of plans for new buildings, installations, equipment, major repairs, and alterations.

8. Be responsible for the development of public relations for the college.

9. Work with other educational institutions in matters of common interests: universities, extension divisions, neighboring colleges, Home Study Institute, hospitals, elementary schools, academies, and other cooperating institutions.

10. Promote the religious life of students, faculty, and staff.

11. Represent the college in the churches and secondary schools of the constituency, and at meetings of educational and accrediting organizations.

12. Assign special duties to administrative officers.

13. Appoint faculty members to serve on standing committees and appoint ad hoc committees whenever necessary. President shall serve as an ex-officio member of all committees.

14. Appoint advisors for student organizations and clubs.

15. Plan with academic dean the in-service training for faculty.

16. Be responsible for developing and promoting plans for student recruitment.

17. Serve as chief disciplinary officer of the college.

18. Be responsible for formation of class organization.

19. Be responsible for planning chapel exercises and special events for college and university.

20. Plan commencement exercises, signing and conferring, together with chairman of board, the professional degrees and diplomas awarded by the college.

21. Maintain faculty and staff personnel records.

22. Be the custodian of the corporate seal.

23. Be the legal and institutional representative of the college, and delegate such representation when necessary. 
24. Assume additional responsibilities appropriate to office as board may authorize.

In addition to the above duties of the presidents, the General Conference's Education Department identified twelve characteristics of a successful Seventh-day Adventist college or university. These characteristics, considered to be the main areas of the chief executive officer's roles and functions, are: (1) Philosophy and mission, (2) Governance and accountability; (3) Administration, (4) Faculty and staff, (5) Programs, (6) Students, (7) Spiritual life, (8) Financial resources, (9) Infrastructure, equipment, and services, (10) Communication, (11) Recruitment and follow-up, and (12) Recognition and accreditation. See Appendix D.

\section{Summary}

The chief executive officers of senior colleges and universities have multiple roles. Their titles vary from institution to institution and from country to country. They are called "president," "chancellor," "vice chancellor," "rector," "dean," and "principal." They are the chief executives of the institutions and their boards. They have other administrative officers who look after their respective areas of responsibilities. There are areas of disagreement and agreement in perception and expectation of certain matters of leadership functions. 
CHAPTER III

\section{METHODOLOGY}

\section{Introduction}

In the previous chapter the review of pertinent literature indicated that there is a difference in perception held by various leaders on the administrative team about senior college and university presidents' role performances. The major purpose of this study was to determine the extent of actual role performance in the 2004-2005 school year as compared to expected role responsibilities of presidents of Seventh-day Adventist senior colleges and universities around the world. Another purpose was to determine whether there were any differences in perception between the actual and expected roles of SDA presidents, as viewed by the five status groups, namely, board chairs, presidents, academic vice presidents, student service vice presidents, and finance vice presidents of Seventh-day Adventist senior colleges and universities around the world.

This chapter deals with the research methodology used in this study. It contains the research design, selection of the population and sample, format and content description of the survey instrument, variables, research questions and their corresponding null hypotheses, data collection strategies, statistical analysis methods, reliability and validity of instrument, generalizability, and a chapter summary. 


\section{Research Design}

This study was descriptive and exploratory in nature. It employed a survey method and a quantitative statistical analysis procedure. It was descriptive because it did "describe systematically the facts and characteristics of a given population or area of interest" and such research involves a database. The design was exploratory because for the first time it explored some of the variables that were not investigated before. This research was non-experimental because, according to McMillan and Schumacher, there was "no manipulation of conditions," rather it "made observations" and "obtained measures from subjects to describe something that has happened."

\section{Population and Sample}

At the time of this study there were twenty-eight senior colleges and universities, accredited by the Adventist Accrediting Association (AAA) ${ }^{3}$ as graduate institutions that were operated by the Seventh-day Adventist world church in seventeen countries, including the U.S.A. in the 2004-2005 school year. These institutions are located in eleven world geographic and administrative regions, called divisions, as identified by the Seventh-day Adventist world church. Table 1 provides the target population of the five status groups of this study listed by the Seventh-day Adventist world church's administrative divisions, countries, and institutions. These five status groups are: (1) 18.

'S. Isaac and W. Michael, Handbook in Research and Evaluation (San Diego, CA: EDITS, 1979),

${ }^{2}$ James H. McMillan and Sally Schumacher, Research in Education: A Conceptual Introduction, $3^{\text {rd }}$ ed. (New York, NY: HarperCollins College Publishers, 1993), 32.

${ }^{3}$ Seventh-day Adventist Yearbook 2006, 385-501. 
chairs of boards of trustees (CBT), (2) presidents, who are chief executive officers

(CEO), (3) academic vice presidents, who are chief academic officers (CAO), (4) student

service vice presidents, who are chiefs of student service (CSS), and (5) finance vice

presidents, who are chief financial officers (CFO).

Table 1. World divisions, numbers of countries, colleges/universities, and target status groups represented

\begin{tabular}{|c|c|c|c|c|c|c|c|c|}
\hline \multirow{2}{*}{$\begin{array}{c}\text { Administrative Divisions } \\
\text { of Seventh-day Adventist } \\
\text { World Church } \\
\text { Represented }\end{array}$} & \multirow{2}{*}{$\mid \begin{array}{c}\text { Number } \\
\text { of } \\
\text { Countries } \\
\text { Represented }\end{array}$} & \multirow{2}{*}{$\begin{array}{c}\text { Number of } \\
\text { Colleges/ } \\
\text { Universities } \\
\text { Represented }\end{array}$} & \multicolumn{6}{|c|}{ Number of Target Population } \\
\hline & & & $\mathrm{CBT}$ & CEO & CAO & CSS & CFO & Total \\
\hline 1. East Central Africa & 1 & 1 & 1 & 1 & 1 & 1 & 1 & 5 \\
\hline 2. Euro-Africa & 2 & 2 & 2 & 2 & 2 & 2 & 2 & 10 \\
\hline 3. Euro-Asia & 1 & 1 & 1 & 1 & 1 & 1 & 1 & 5 \\
\hline 4. Inter-America & 2 & 2 & 2 & 2 & 2 & 2 & 2 & 10 \\
\hline 5. North America & 2 & 11 & 11 & 11 & 11 & 11 & 11 & 55 \\
\hline 6. Northern Asia Pacific & 1 & 1 & 1 & 1 & 1 & 1 & 1 & 5 \\
\hline 7. South America & 3 & 3 & 3 & 3 & 3 & 3 & 3 & 15 \\
\hline 8. South Pacific & 2 & 2 & 2 & 2 & 2 & 2 & 2 & 10 \\
\hline 9. Southern Africa-Indian & 1 & 1 & 1 & 1 & 1 & 1 & 1 & 5 \\
\hline 10. Southern Asia & 1 & 1 & 1 & 1 & 1 & 1 & 1 & 5 \\
\hline 11. Southern Asia-Pacific & 1 & 3 & 3 & 3 & 3 & 3 & 3 & 15 \\
\hline TOTAL & 17 & 28 & 28 & 28 & 28 & 28 & 28 & 140 \\
\hline
\end{tabular}

Note: $\mathrm{CBT}=$ Chair of Board of Trustees; $\mathrm{CEO}=$ Chief Executive Officer (president, chancellor, vicechancellor, rector, principal, etc.); $\mathrm{CAO}=$ Chief Academic Officer (academic vice president/academic dean, etc.); CSS $=$ Chief of Student Service (student service vice president/dean of students, etc.); $\mathrm{CFO}=$ Chief Financial Officer (finance vice president, dean of finance, director of finance, etc.). 
The sample for this study included subjects who responded to the research questionnaires. A total of 82 people returned the completed survey instrument. Four respondents, who did not meet the inclusion criteria, were disqualified, thereby leaving a total sample of 78 respondents in the said five status groups. The sample consisted of 18 board chairs, 17 presidents, 16 academic vice presidents, 13 student service vice presidents, and 14 finance vice presidents from 27 Adventist Accrediting Associationaccredited centers of higher education located in 16 countries.

\section{Instrumentation}

The participants in this study were requested to respond to forty-seven questions in the questionnaires constructed primarily with close-ended questions. The open-ended questions at the end of each section of the questionnaire, however, gave respondents the opportunity to express themselves in their own words regarding role performance and role expectation of the presidents. For each question there are two grids, marked Actual Role Performance and Expected Role Responsibility.

The questionnaire elicited data in understanding the role and functions of the president as he or she actually performed at the time of the survey. Seven broad categories of presidential functions listed on seven pages have positively-stated questions that asked the status groups to give their views on the first grid regarding their perceptions as to how their president actually performed his or her job. On the second grid they indicated on the same set of questions their expectations of what the president should do. Each function has values of 1 to 5. While 1 represents strong disagreement, 
5 means strong agreement. The respondents were expected to circle the numerals on these two grids that indicate their perceptions and expectations of the president's role as described in the activity statements. See instrument with direction in Appendix F.

\section{Procedure}

The inclusion of items in the questionnaire of this study was based on a review of the literature and the faculty handbooks received from the Seventh-day Adventist and non-Seventh-day Adventist colleges and universities where presidents' roles, functions, and job descriptions were listed. The non-Seventh-day Adventist colleges and universities, including Catholic, Protestant, and public institutions, were purposely selected. Letters requesting for presidents' job descriptions were sent to an equal number of these colleges and universities.

The review of the literature indicates a number of recurring themes relating to the role of the president, and these adequately establish the basis for the questionnaire. The six generally accepted areas of the chief administrator's duties in the areas of academics, finance, faculty, student, community and alumni, and general administration, as suggested by Corson ${ }^{4}$ and others, were selected for inclusion in the questionnaire. Since the Seventh-day Adventist colleges and universities are Christian institutions and have a definitive purpose to exist, the spiritual area of the presidents' roles is a distinctive aspect. The questionnaire on the spiritual role of presidents developed for this study was based on the faculty handbooks received from the Seventh-day Adventist colleges and 58-71.

${ }^{4} \mathrm{~J}$ ohn J. Corson, Governance of College and Universities (New York, NY: McGraw-Hill, 1960), 
universities worldwide, and the published Seventh-day Adventist literature.

\section{Related Studies Investigated}

An Internet search of the Dissertation Abstract Index was carried out to identify studies on role perception of college presidents. Only nine studies were identified. After abstracts from several of these studies were reviewed, two studies, Oliver ${ }^{5}$ and Silas, ${ }^{6}$ were initially selected for possible instrument adaptation.

\section{Validity and Reliability of Instrument}

About 40 percent of the items on the questionnaire in this study were taken from the Oliver and Silas instruments. Their instruments were pilot tested and validated. The Oliver instrument went through three major pre-test exercises. First, it was given to four professors of higher education to determine the content validity. They criticized the preliminary questionnaire on the following points: (1) length, (2) scope, (3) content validity, and (4) directions. They inserted various changes and another revised questionnaire, broader in scope with a variety of formats which required different types of responses, was developed. This instrument had greater depth and it structured items based on the president's administrative functions. The Oliver questionnaire consisting of 101 items was organized in the following format:

5Arnold Robert Oliver, "The University Presidency as Perceived by Boards of Trustees, Presidents, and Academic Vice Presidents in Selected Colleges and Universities" (Ph.D. dissertation, Southern Illinois University, 1972).

${ }^{6}$ Samuel L. Silas, "Role expectations of Presidents in Predominantly Black State and Privately Supported Colleges and Universities as Perceived by Trustees, Presidents, and Deans" (Ph.D. dissertation, Southern Illinois University, 1974). 
Part 1: Demographic Data (8 items)

Part 2: Perceptions of the Role and Philosophy of the President (30 items)

Part 3: Necessary Background for a President (15 items)

Part 4: Perceptions of Specific Actions of a President (30 items)

Part 5: Presidential Interaction (10 items)

Part 6: Forced Ratings by Presidents regarding their functions in terms of importance, preference, and success ( 3 items)

Optional: Open-ended questions (5 items).

Second, this refined instrument was reviewed by a group of ten advanced doctoral students in the field of higher education who completed the questionnaire. Several adjustments were made as a result of this session. They were:

1. Average completion time for the questionnaire (excluding open-ended questions) was 22.4 minutes.

2. The wording of some questions was reduced.

3. Directions were included as to how a response should be marked.

4. Numerical values in the rating scale were inverted.

5. Overview of directions on the first page was modified and directions for each section were inserted.

6. The cover letter was shortened.

7. On three questions, greater specificity of wording was recommended for clarification of the intent.

Lastly, the final step of the instrument revision done by Oliver was the 
development of a third form of the questionnaire which was used as a basis of dialogue with twelve administrators and board members. Each trustee, president, and dean was selected to test the content validity of the instrument. Each of the interviews followed as structured a pattern as possible. All the administrators and board members had examined the questionnaire prior to the interview. All of the suggestions were then incorporated into the questionnaire.

The Silas instrument was slightly modified in that it had some supplemental questions. Although the Silas instrument was already validated, the questionnaire was examined by a number of individuals. The extensive review of the literature and the examination of the instrument by individuals associated with and familiar with the role of the college and university president lent added credibility to the content validity of the instrument.

The remaining 60 percent of the questionnaire items in this study were developed based on the faculty handbooks of Seventh-day Adventist colleges and universities, and Seventh-day Adventist literature on higher education. There are forty-seven close-ended questions, and seven open-ended questions--one at the end of each of the seven sections.

\section{Preliminary Instrument}

The preliminary instrument of this study was modified from the Oliver and the Silas instruments. I developed this under the guidance of the dissertation committee. The instrument had two parts. Part I called for demographic information. Part II contained the survey questionnaire. The questionnaire was based primarily on the faculty 
handbooks received from the 22 Seventh-day Adventist senior colleges and universities worldwide, two Protestant colleges and universities, two Catholic universities, and one Ivy League university. It also took into consideration the Seventh-day Adventist publications and literature on the president's duties in higher education. This preliminary instrument contained 44 statements distributed in seven categories of the president's functions.
I. Student-Related Functions ( 7 items)
II. Faculty-Related Functions (8 items)
III. General Administration (10 items)
IV. Finance-Related Functions (5 items)

V. Physical Facilities-Related Functions (6 items)

VI. Community Relations and Alumni-Related Functions (5 items), and

VII. Spiritual Life-Related Functions ( 3 items).

Each of the seven broad categories of functions had a specified number of positively-stated statements in the middle of the page with two grids, titled Expected Role Responsibility and Perceived Role Performance appearing on the left and on the right sides of the page, respectively. Each statement was to be weighted on a five-point Likert scale in these two grids to measure the president's perceived role performance and his or her expected roles. The scales were: $1=$ Strongly Disagree, $2=$ Mildly Disagree, $3=$ No Opinion, $4=$ Mildly Agree, and $5=$ Strongly Agree .

The preliminary instrument and directions were sent out with a cover letter to 15 administrators, representing eight institutions in seven countries of six world divisions. 
The participants were not part of the five status groups, who received the final questionnaires. The selection of the preliminary participants was based on their professional backgrounds serving as presidents, academic deans, chief financial officers, deans of students, administrative team members, department heads, and board chairperson in the Seventh-day Adventist senior colleges and universities, who had a thorough knowledge in the area of presidents' functions and were, therefore, fully qualified to validate the instrument. See Appendix E.

The purposes of the preliminary instrument were (1) to obtain advice that would improve the instrument of research; (2) to determine whether or not the questionnaire items were clear and understood by the participants; (3) to evaluate the relevance, appropriateness, and effectiveness of the questionnaire in securing a response from the specified respondents; (4) to know from the participants what portions of the questionnaire were to be eliminated and what new functions, or wordings, or ideas were to be added; (5) to be aware of the ease of the participants in responding to the questionnaire; and (6) to receive assistance for validation of the research instrument. About a month later, a follow-up reminder was given to one executive in the United States who had failed to respond the first time.

\section{Modified Instrument}

Out of a total of 15 participants in the preliminary instrument study, 14 of them returned their completed questionnaires along with their comments. The remaining one participant did not answer the 44 questions in Part II but he, like others, provided valuable 
suggestions regarding the scale, content, wordings, and additions and deletions of some role items. I shared the opinions of these questionnaire participants with my dissertation committee, which, in turn, guided me in the revision of the instrument. The revised instrument was seen by the dissertation committee and a few other scholars, administrators, and researchers in the field of educational administration. They expressed their opinions that the instrument was adequate, relevant, and reliable to measure the actual and expected roles of the presidents as stated in the questionnaire of this research. Appendix $\mathrm{F}$ shows the modifications of the final instrument over the preliminary instrument in Appendix E as follows:

1. The demographic section in Part I was completely eliminated from the instrument because it was found out that the research did not call for findings related to the variables.

2. The scale was modified as a continuum. The measures of the scale were modified too. The word "mildly" was eliminated. Instead of "no opinion," "neither agree nor disagree" was suggested to be the midpoint scale in the instrument.

3. The format of the final instrument was changed from that of the preliminary instrument.

4. In the preliminary instrument there were forty-four questions. The preliminary instrument participants retained 40 items out of 44 items with some modifications and added 7 more new items, making a total of 47 questions in the final instrument in seven broad categories of functions.

Four items were dropped from the preliminary questionnaire. Those were: 
General Administration: Item 4, "Bear responsibility for satisfactory governance of the institution," Item 5, "Serve as an ex-officio member of all faculty committees," Item 7, "Assign officers their roles, and Item 10, "Serve as chief disciplinary officer."

Seven new items were added to the final questionnaire. Those were: StudentRelated Functions: Item 8. "Serve as mentor of students."

General Administrative Functions: Item 22, "Foster research and intellectual climate for the institution," Item 23 , "Comply with legal mandates and laws in addressing grievances," and Item 25, "Delegate responsibility, authority, and needed resources to appropriate administrators and support their roles."

Community- and Alumni-Related Functions: Item 4, "Listen to constituency and alumni."

Spiritual Life-Related Functions: Items 43 and 44 were Item 1 in the preliminary questionnaire, and Item 47, "Focus on the Christian philosophy of the institution.

The revised, modified, final instrument has the same seven broad categories of the president's functions as found in the preliminary instrument:

I. Student-Related Functions (8 items)

II. Employee-Related Functions (8 items)

III. General Administrative Functions (9 items)

IV. Finance-Related Functions (5 items)

V. Physical Facilities-Related Functions (6 items)

VI. Community- and Alumni-Related Functions (6 items), and

VII. Spiritual Life-Related Functions (5 items). 
5. Deletion, addition, alteration, and modifications in the statements were done according to the feedback from the qualified preliminary questionnaire participants. Some of their comments, suggestions and views have been summarized in Appendix E.

An internal consistency analysis was attempted using a sample of 78 educators in five status groups that participated in the study. The Statistical Package for the Social Sciences (SPSS), version 12, has produced results showing the reliability estimates of the actual and the expected role scales for the 47 items by seven broad categories of the president's functions.

\section{Reliability}

The total scale as well as the subscale reliability estimates for the instrument used in this study are shown in Table 2. The Cronbach's Alpha coefficient determines the degree of homogeneity of the items within each scale and shows the extent to which each scale is internally consistent.

Table 2 shows that the total scale reliability is excellent at 0.93 for actual roles and 0.92 for expected roles, suggesting high internal consistency. The Alpha reliability for Actual Roles subscales ranges from a low of 0.74 for finance-related responsibilities to a high of 0.90 for physical facilities-related responsibilities. For Expected Roles, the reliability estimates range from a low of 0.58 for general administrative responsibilities to a high of 0.87 for physical facilities-related responsibilities.

Two expected role scales, such as general administrative responsibilities with an alpha of 0.58 , and community- and alumni-related responsibilities with an alpha of 0.59 , 
have reliability estimates slightly below 0.60 . These two scales should be interpreted with caution. However, these estimates appear adequate for this type of measure where "studies of groups can tolerate a lower reliability, sometimes as low as 0.50 in exploratory research."

Table 2. Internal consistency reliability estimates of role scales

\begin{tabular}{|c|c|c|c|c|c|}
\hline \multirow[b]{2}{*}{ Scale } & \multirow[b]{2}{*}{ Sub-scale } & \multirow[b]{2}{*}{ No. of Items } & \multirow[b]{2}{*}{$n$} & \multicolumn{2}{|c|}{ Cronbach's Coefficient Alpha } \\
\hline & & & & Actual & Expected \\
\hline $\begin{array}{l}\text { I. Student-Related } \\
\text { Questionnaire }\end{array}$ & Items $1-8$ & 8 & 78 & 0.80 & 0.83 \\
\hline $\begin{array}{l}\text { II. Employee-Related } \\
\text { Questionnaire }\end{array}$ & Items $9-16$ & 8 & 78 & 0.82 & 0.81 \\
\hline $\begin{array}{l}\text { III. General Administrative } \\
\text { Questionnaire }\end{array}$ & Items $17-25$ & 9 & 78 & 0.75 & 0.58 \\
\hline $\begin{array}{l}\text { IV. Finance-Related } \\
\text { Questionnaire }\end{array}$ & Items $26-30$ & 5 & 78 & 0.74 & 0.71 \\
\hline $\begin{array}{l}\text { V. Physical Facilities- } \\
\text { Related Questionnaire }\end{array}$ & Items $31-36$ & 6 & 78 & 0.90 & 0.87 \\
\hline $\begin{array}{l}\text { VI. Community- and Alumni- } \\
\text { Related Questionnaire }\end{array}$ & Items $37-42$ & 6 & 78 & 0.77 & 0.59 \\
\hline $\begin{array}{l}\text { VII. Spiritual Life-Related } \\
\text { Questionnaire }\end{array}$ & Items $43-47$ & 5 & 78 & 0.84 & 0.73 \\
\hline$\overline{\text { Total }}$ & & 47 & 78 & 0.93 & 0.92 \\
\hline
\end{tabular}

Note: Scaling method: $1=$ Strongly Disagree, $2=$ Disagree, $3=$ Neither Agree nor Disagree, $4=$ Agree, and $5=$ Strongly Agree. The subscale items are found in the seven-part research instrument in Appendix F.

\section{Methods}

The following methods were used in this study:

The Oliver and Silas instruments were modified and some supplemental questions

${ }^{7}$ James H. McMillan and Sally Schumacher, Research in Education: A Conceptual Introduction, $3^{\text {rd }}$ ed. (New York, NY: HarperCollins, 1993), 231. 
were added which I believed were essential to the fulfillment of the purpose of this study.

The modified instrument was evaluated by 15 people for evaluation and validation who either served or were still serving as board chairperson, president, vice president for academic affairs or academic dean, vice president for student service or dean of students, and vice president for finance. All of them served at Seventh-day Adventist senior colleges and universities. The lists of the research instrument evaluation and validation study participants are included in Appendix E.

An eight-page color-coded questionnaire, including a direction page, was mailed on September 23, 2004, to 140 subjects in 28 senior colleges and universities in 17 countries around the world, along with an endorsement letter from the Education Director of the Seventh-day Adventist world church with headquarters in Maryland, USA. The five groups selected as participants in the study were: (1) 28 chairpersons of boards of trustees, (2) 28 presidents, or vice-chancellor, or chancellor, or principal, or rector, (3) 28 academic vice presidents, (4) 28 student service vice presidents, or deans of students, and (5) 28 finance vice presidents, or chief financial officer. The color-code method was adopted for identification of status groups and follow-up and for maintaining anonymity of respondents by particular institutions and individuals.

A number of reminders were sent out to the subjects in various countries. The dates of these reminders were September 28, 2004; October 17, 2004; October 19, 2004; January 11, 2005; and February 14, 2005. Some subjects received five reminders, some four, some three, and some two. The reminders were sent based on response and nonresponse rates. Since this study involved countries outside of the U.S.A., responses were 
slow and not 100 percent return rate. Several reminders were given in various forms, such as regular mail, email, fax, courier service, hand-delivery, etc.

\section{Collection of Data}

The subjects responded to 47 questions on a continuum scale of 1 to 5 , indicating their perceptions of presidents' actual role performances by circling any one number for each role item. The numbers represented as follows: $1=$ Strongly Disagree (SD), $2=$ Disagree (D), $3=$ Neither Agree nor Disagree (N), $4=$ Agree (A), and $5=$ Strongly Agree (SA). The respondents followed the same procedure answering the questions on their degrees of expectation of presidents' role responsibilities. See Appendix F.

The completed questionnaires from the U.S.A. and Puerto Rico were returned to me by the subjects in the postage-stamped envelopes that I provided. The subjects in foreign countries returned their completed questionnaires in the envelopes I provided but they affixed their countries' postage stamps.

When the envelopes containing the completed questionnaires arrived, my assistant unsealed them, marked the receipt checksheet, and stacked the questionnaires and envelopes separately. This process not only ensured the privacy and anonymity of the respondents, but it also helped in the data collection follow-up strategy.

\section{Processing of Data}

A one-way Analysis of Variance (ANOVA) was used for each of the forty-seven variables regarding the presidents' actual role performances and expected role responsibilities to process and interpret the responses from the survey questionnaires. 
The SPSS, version 12, was used for processing, treatment, and analysis of data. Answers to five research questions mentioned in chapter 1 were obtained by testing the three null hypotheses. The level of significance was set at 0.05 . However, to control for Type I Error since there were many multiple comparisons, a corrected level of significance following the Bonferroni method was used. ${ }^{8}$ This corrected level of significance was $0.05 / 47$, which is approximately $0.001 .^{9}$ If it was significant at the 0.001 level, the hypothesis was deemed significant and the null hypothesis was rejected. If the null hypothesis was not significant at the 0.001 level, it was retained.

\section{Research Questions}

The statistical approach to the following five research questions based on the purpose of the study as stated in chapter 1 guided in the investigation:

Research Question 1: What are the actual role performances of presidents of Seventh-day Adventist senior colleges and universities around the world, as perceived by their board chairs, presidents, academic vice presidents, student service vice presidents, and finance vice presidents?

Research Question 2: What are the expected role responsibilities of presidents of Seventh-day Adventist senior colleges and universities around the world, as viewed by their board chairs, presidents, academic vice presidents, student service vice presidents, and finance vice presidents?

${ }^{8}$ Barbara G. Tabachnick and Linda S. Fiddel, Using Multivariate Statistics (Boston, MA: Pearson Education, 2007), 402, 407.

${ }^{9}$ Samuel B. Green and Neil J. Salkind, Using SPSS for Windows and Macintosh: Analyzing and Understanding Data (Upper Saddle River, NJ: Prentice Hall, 2007), 204. 
Research Question 3: Is there a difference in perception between groups of board chairs, presidents, academic vice presidents, student service vice presidents, and finance vice presidents of Seventh-day Adventist senior colleges and universities around the world regarding their college/university presidents' actual role performances?

Research Question 4: Is there a difference in expectations between groups of board chairs, presidents, academic vice presidents, student service vice presidents, and finance vice presidents of Seventh-day Adventist senior colleges and universities around the world regarding their college/university presidents' expected role responsibilities?

Research Question 5: Is there a difference in perception and expectation between actual role performances and expected role responsibilities of presidents of Seventh-day Adventist senior colleges and universities around the world, as perceived and expected by their groups of board chairs, presidents, academic vice presidents, student service vice presidents, and finance vice presidents?

\section{Related Null Hypotheses and Their Statistical Tests}

The following research hypotheses stated in null form were tested:

Null Hypothesis 1: There is no difference among the status groups (board chairs, presidents, academic vice presidents, student service vice presidents, and finance vive presidents) on each of the perceived actual role performances of presidents of Seventhday Adventist senior colleges and universities around the world.

A one-way ANOVA was used to test this null hypothesis to see if any differences of perceptions existed between the groups on the actual roles of the presidents. 
Null Hypothesis 2: There is no difference among the status groups (board chairs, presidents, academic vice presidents, student service vice presidents, and finance vice presidents) on each of the expected roles of presidents of Seventh-day Adventist senior colleges and universities around the world.

A one-way ANOVA was used to test this null hypothesis to detect if any differences in expectations existed between the groups on the expected roles of the presidents.

Null Hypothesis 3: There is no difference between the perceived actual role performances and expected role responsibilities of presidents of Seventh-day Adventist senior colleges and universities around the world, as viewed by each of the status groups (board chairs, presidents, academic vice presidents, student service vice presidents, and finance vice presidents).

A two-tailed paired samples $t$-test was used to test this null hypothesis to determine if any differences existed between the perceived actual role performances and expected roles of the presidents.

\section{Summary}

This chapter was concerned with the research design and methodology to answer the five research questions. Included in the study were the selection of the population and sample, description of the instrument, statement of the related null hypotheses, and collection of data. It also introduced the application of appropriate statistical procedures to test the three null hypotheses. 


\section{CHAPTER IV}

\section{PRESENTATION AND ANALYSIS OF DATA}

\section{Introduction}

This chapter deals with sample characteristics, data presentation, data analysis, testing of null hypotheses, and statistical results. The primary purpose, described in chapter 1 of this study, was to investigate the actual role performances and the expected role responsibilities of the presidents of the Seventh-day Adventist senior colleges and universities as perceived by the five status groups and also to identify areas of common agreement and disagreement in the role perceptions and role expectations. The following research questions were addressed in this study:

Research Question 1: What are the actual role performances of presidents of Seventh-day Adventist senior colleges and universities around the world, as perceived by their board chairs, presidents, academic vice presidents, student service vice presidents, and finance vice presidents?

Research Question 2: What are the expected role responsibilities of presidents of Seventh-day Adventist senior colleges and universities around the world, as viewed by their board chairs, presidents, academic vice presidents, student service vice presidents, and finance vice presidents? 
Research Question 3: Is there a difference in perception between groups of board chairs, presidents, academic vice presidents, student service vice presidents, and finance vice presidents of Seventh-day Adventist senior colleges and universities around the world regarding their college/university presidents' actual role performances?

Research Question 4: Is there a difference in expectation between groups of board chairs, presidents, academic vice presidents, student service vice presidents, and finance vice presidents of Seventh-day Adventist senior colleges and universities around the world regarding their college/university presidents' expected role responsibilities?

Research Question 5: Is there a difference in perception and expectation between actual role performances and expected role responsibilities of presidents of Seventh-day Adventist senior colleges and universities around the world, as perceived and expected by their groups of board chairs, presidents, academic vice presidents, student service vice presidents, and finance vice presidents?

Research Question 1 and Research Question 2 were answered by descriptive statistics examining the means, which were obtained using SPSS, version 12. Research Questions 3, 4, and 5 have three related null hypotheses.

Null Hypothesis 1: There is no difference among the status groups (board chairs, presidents, academic vice presidents, student service vice presidents, and finance vice presidents) on each of the perceived actual role performances of presidents of Seventhday Adventist senior colleges and universities around the world. A one-way ANOVA was used to determine any differences among the means on the perceived actual role performances of the presidents. 
Null Hypothesis 2: There is no difference among the status groups (board chairs, presidents, academic vice presidents, student service vice presidents, and finance vice presidents) on each of the expected roles of presidents of Seventh-day Adventist senior colleges and universities around the world. A one-way ANOVA was used to see whether any differences among the means on the expected roles of the presidents existed or not.

Null Hypothesis 3: There is no difference between the perceived actual role performances and expected role responsibilities of presidents of Seventh-day Adventist senior colleges and universities around the world, as viewed by each of the status groups (board chairs, presidents, academic vice presidents, student service vice presidents, and finance vice presidents). A two-tailed paired samples $t$-test was used to test this null hypothesis to detect any differences between actual and expected roles.

\section{Sample Characteristics}

The purposive sample of 140 administrators included 28 board chairs, 28 presidents, 28 academic vice presidents, 28 student service vice presidents, and 28 finance vice presidents, and was drawn from the 28 Seventh-day Adventist senior colleges and universities located in 17 countries of the world's 11 administrative divisions or geographic regions as determined by the Seventh-day Adventist world church. These 28 institutions of higher education were in operation during the 2004-2005 academic year and were included in the study. Table 3 presents these graduate degreeoffering institutions grouped by administrative divisions and countries, as listed in the Seventh-day Adventist Yearbook 2006. 
Table 3. Colleges/universities and countries included in the study by divisions

\begin{tabular}{llr}
\hline Division & College/University & Country \\
\hline East Central Africa Division & University of Eastern Africa, Baraton & Kenya \\
Euro-Africa Division & Friedensau Adventist University & Germany \\
& Saleve Adventist University & France \\
Euro-Asia Division & Zaoksky Adventist University & Russian Federation \\
Inter-America Division & Antillian Adventist University & Puerto Rico \\
& Montemorelos University & Mexico \\
North America Division & Andrews University & U.S.A. \\
& Atlantic Union College & U.S.A. \\
& Canadian University College & Canada \\
& La Sierra University & U.S.A. \\
& Loma Linda University & U.S.A. \\
& Pacific Union College & U.S.A. \\
& Southern Adventist University & U.S.A. \\
& Southwestern Adventist University & U.S.A. \\
& Union College & U.S.A. \\
& Walla Walla University & U.S.A. \\
& Washington Adventist University & U.S.A. \\
& Sahmyook University & South Korea \\
Northern Asia-Pacific Division & Brazil \\
South America Division & Brazil Adventist University, Campus 1 & Argentina \\
South Pacific Division & River Plate Adventist University & Australia \\
& Avondale College & Simbabwe \\
Southern Africa-Indian Division & Solusi University University & India \\
Southern Asia Division & Spicer Memorial College & Philippines \\
Southern Asia-Pacific Division & Adventist International Institute of Advanced Studies & Philippines \\
& Adventist University of the Philippines & Philippines \\
& Northern Luzon Adventist College &
\end{tabular}

Table 4 shows the number and percentages of various countries participated in the study by the status groups. Out of the 17 countries chosen, the participating groups from $16(94.1 \%)$ countries have responded to the study. Eleven of these countries $(64.7 \%)$ were represented by the chairs of boards of trustees and academic vice presidents, 8 countries $(47.1 \%)$ were represented by the presidents and student service vice presidents, and 10 countries $(58.8 \%)$ were represented by the finance vice presidents, representing a total of 27 Seventh-day Adventist senior colleges and universities worldwide. 
Table 4. Participating countries by status groups

\begin{tabular}{|c|c|c|c|c|c|}
\hline \multirow[b]{2}{*}{ Country } & \multicolumn{5}{|c|}{ Status Groups } \\
\hline & $\begin{array}{l}\text { Board } \\
\text { Chair }\end{array}$ & President & $\begin{array}{l}\text { Academic } \\
\text { VP }\end{array}$ & $\begin{array}{l}\text { Student } \\
\text { Service VP }\end{array}$ & $\begin{array}{c}\text { Finance } \\
\text { VP }\end{array}$ \\
\hline 1. Argentina & & & $x$ & & $x$ \\
\hline 2. Australia & $x$ & $x$ & $x$ & & \\
\hline \multicolumn{6}{|l|}{ 3. Bolivia* } \\
\hline 4. Brazil & $x$ & & & $x$ & $x$ \\
\hline 5. Canada & $x$ & $x$ & $x$ & $x$ & $x$ \\
\hline 6. France & & $x$ & - & & \\
\hline 7. Germany & $x$ & $x$ & & & $x$ \\
\hline 8. India & $x$ & & $x$ & $x$ & \\
\hline 9. Kenya & & & $x$ & $x$ & \\
\hline 10. Korea & $x$ & $x$ & $x$ & & $x$ \\
\hline 11. Mexico & $x$ & $x$ & $x$ & $x$ & $x$ \\
\hline 12. Papua New Guinea & & & $x$ & $x$ & \\
\hline 13. Philippines & $x$ & $x$ & $x$ & & $x$ \\
\hline 14. Puerto Rico & $x$ & & & $x$ & $x$ \\
\hline 15. Russian Federation & & & $x$ & & $x$ \\
\hline 16. U.S.A. & $x$ & $x$ & $x$ & $x$ & $x$ \\
\hline 17. Zimbabwe & $x$ & & & & \\
\hline No. of countries responding & 11 & 8 & 11 & 8 & 10 \\
\hline Percentage of countries responding & 64.7 & 47.1 & 64.7 & 47.1 & 58.8 \\
\hline
\end{tabular}

$X$ Denotes status groups representing the countries.

*Did not respond.

Table 5 presents the number of subjects and respondents by senior college and university. Out of a sample of 140 administrators, a total of 82 administrative team members responded (58.6\%) to the survey. Responses of 78 participants from 27 institutions out of 28 institutions have been included in this study. 
Table 5. Institutions and their response rates

\begin{tabular}{|c|c|c|c|c|}
\hline Name of Institution and Its Place of Location & $\begin{array}{c}\text { Number } \\
\text { of } \\
\text { Samples }\end{array}$ & \begin{tabular}{|l|} 
Number of \\
Subjects \\
Responded
\end{tabular} & $\begin{array}{c}\text { Percentage } \\
\text { of } \\
\text { Response }\end{array}$ & $\begin{array}{l}\text { Percentage } \\
\text { of } \\
\text { Response } \\
\text { Included }\end{array}$ \\
\hline $\begin{array}{l}\text { 1. Adventist International Institute of Advanced Studies, } \\
\text { Silang, Cavite, Philippines }\end{array}$ & 5 & 2 & 40.0 & 40.0 \\
\hline $\begin{array}{l}\text { 2. Adventist University of the Philippines } \\
\text { Silang Cavite, Philippines }\end{array}$ & 5 & 2 & 40.0 & 40.0 \\
\hline 3. Andrews University, Berrien Springs, MI, USA & 5 & 4 & 80.0 & 80.0 \\
\hline $\begin{array}{l}\text { 4. Antillian Adventist University } \\
\text { Bo. La Quinta, Mayaguez, Puerto Rico }\end{array}$ & 5 & 3 & 60.0 & 60.0 \\
\hline 5. Atlantic Union College, South Lancaster, MA, USA & 5 & 3 & 60.0 & 60.0 \\
\hline 6. Avondale College, Cooranbong, NSW, Australia & 5 & $3\left(2^{*}\right)$ & 60.0 & 40.0 \\
\hline $\begin{array}{l}\text { 7. Bolivia Adventist University } \\
\text { Vinto, Cochabamba, Bolivia }\end{array}$ & 5 & 0 & 00.0 & 00.0 \\
\hline 8. Brazil Adventist University, Sao Paulo, SP, Brazil & 5 & 3 & 60.0 & 60.0 \\
\hline 9. Canadian University College, Lacombe, AB, Canada & 5 & 5 & 100.0 & 100.0 \\
\hline $\begin{array}{l}\text { 10. Friedensau Adventist University } \\
\text { An derlhle 5a, Friedensau, Germany }\end{array}$ & 5 & 3 & 60.0 & 60.0 \\
\hline 11. La Sierra University, Riverside, CA, USA & 5 & 4 & 80.0 & 80.0 \\
\hline 12. Loma Linda University, Loma Linda, CA, USA & 5 & 5 & 100.0 & 100.0 \\
\hline $\begin{array}{l}\text { 13. Montemorelos University } \\
\text { Poniente, Montemorelos, N.L., Mexico }\end{array}$ & 5 & 5 & 100.0 & 100.0 \\
\hline $\begin{array}{l}\text { 14. Northern Luzon Adventist College } \\
\text { Sison, Pangasinan, Philippines }\end{array}$ & 5 & 1 & 20.0 & 20.0 \\
\hline $\begin{array}{l}\text { 15. Pacific Adventist University } \\
\text { Port Moresby, Papua New Guinea }\end{array}$ & 5 & 2 & 40.0 & 40.0 \\
\hline 16. Pacific Union College, Angwin, CA, USA & 5 & 3 & 60.0 & 60.0 \\
\hline $\begin{array}{l}\text { 17. River Plate Adventist University } \\
\text { Libertador San Martin, Entre Rios, Argentina }\end{array}$ & 5 & $2\left(1^{*}\right)$ & 40.0 & 20.0 \\
\hline 18. Sahmywook University, Nowon-gu, Seoul, S. Korea & 5 & 4 & 80.0 & 80.0 \\
\hline $\begin{array}{l}\text { 19. Saleve Adventist University } \\
\text { Chemin du Perouzet, Collonges-sous-Saleve, France }\end{array}$ & 5 & 1 & 20.0 & 20.0 \\
\hline 20. Solusi University, Solusi, Bulawayo, Zimbabwe & 5 & 1 & 20.0 & 20.0 \\
\hline $\begin{array}{l}\text { 21. Southern Adventist University } \\
\text { Collegedale, TN, USA } \\
\end{array}$ & 5 & 3 & 60.0 & 60.0 \\
\hline $\begin{array}{l}\text { 22. Southwestern Adventist University } \\
\text { West Hillcrest, Keene, TX, USA } \\
\end{array}$ & 5 & 4 & 80.0 & 80.0 \\
\hline 23. Spicer Memorial College, Ganeshkhind, Pune, India & 5 & 3 & 60.0 & 60.0 \\
\hline 24. Union College, Lincoln, NE, USA & 5 & 4 & 80.0 & 80.0 \\
\hline 25. University of Eastern Africa, Baraton, Eldoret, Kenya & 5 & 2 & 40.0 & 40.0 \\
\hline 26. Walla Walla University, College Place, WA, USA & 5 & 5 & 100.0 & 100.0 \\
\hline $\begin{array}{l}\text { 27. Washington Adventist University } \\
\text { Takoma Park, MD, USA }\end{array}$ & 5 & 3 & 60.0 & 60.0 \\
\hline $\begin{array}{l}\text { 28. Zaoksky Adventist University } \\
\text { Zaosky, Tula Region, Russian Federation }\end{array}$ & 5 & $2\left(0^{* *}\right)$ & 40.0 & 00.0 \\
\hline Totals & 140 & $\begin{array}{c}82 \\
\text { included } 78 \\
\end{array}$ & 58.6 & 57.8 \\
\hline
\end{tabular}

*Denotes number of respondents included in the study.

**Denotes number of respondents not included in the study. 
Table 6 provides a list of five status groups who have responded by institutions. It also provides total number of respondents by groups and colleges and universities.

Table 6. Respondents by status groups and institutions

\begin{tabular}{|c|c|c|c|c|c|c|}
\hline \multirow[b]{2}{*}{ College/ University } & \multicolumn{5}{|c|}{ Respondent Category } & \multirow[b]{2}{*}{ Total } \\
\hline & \begin{tabular}{|l} 
Board \\
Chair
\end{tabular} & President & $\begin{array}{c}\text { Academic } \\
\text { VP }\end{array}$ & $\begin{array}{c}\text { Student } \\
\text { Service } \\
\text { VP }\end{array}$ & $\underset{\text { VP }}{\text { Finance }}$ & \\
\hline $\begin{array}{l}\text { 1. Adventist International Institute of } \\
\text { Advanced Studies }\end{array}$ & 1 & & 1 & & & 2 \\
\hline 2. Adventist University of the Philippines & & 1 & & & 1 & 2 \\
\hline 3. Andrews University & 1 & 1 & & 1 & 1 & 4 \\
\hline 4. Antillian Adventist University & 1 & & & 1 & 1 & 3 \\
\hline 5. Atlantic Union College & & 1 & 1 & & 1 & 3 \\
\hline 6. Avondale College & 1 & 1 & $(1)^{*}$ & & & 2 \\
\hline 7. Brazil Adventist University & 1 & & & 1 & 1 & 3 \\
\hline 8. Canadian University College & 1 & 1 & 1 & 1 & 1 & 5 \\
\hline 9. Friedensau Adventist University & 1 & 1 & & & 1 & 3 \\
\hline 10. La Sierra University & & 1 & 1 & 1 & 1 & 4 \\
\hline 11. Loma Linda University & 1 & 1 & 1 & 1 & 1 & 5 \\
\hline 12. Montemorelos University & 1 & 1 & 1 & 1 & 1 & 5 \\
\hline 13. Northern Luzon Adventist College & & & & & 1 & 1 \\
\hline 14. Pacific Adventist University & & & 1 & 1 & & 2 \\
\hline 15. Pacific Union College & 1 & 1 & 1 & & & 3 \\
\hline 16. River Plate Adventist University & & & 1 & & $(1)^{*}$ & 1 \\
\hline 17. Sahmyook University & 1 & 1 & 1 & & 1 & 4 \\
\hline 18. Saleve Adventist University & & 1 & & & & 1 \\
\hline 19. Solusi University & 1 & & & & & 1 \\
\hline 20. Southern Adventist University & 1 & 1 & 1 & & & 3 \\
\hline 21. Southwestern Adventist University & 1 & 1 & 1 & 1 & & 4 \\
\hline 22. Spicer Memorial College & 1 & & 1 & 1 & & 3 \\
\hline 23. Union College & 1 & 1 & & 1 & 1 & 4 \\
\hline 24. University of Eastern Africa, Baraton & & & 1 & 1 & & 2 \\
\hline 25. Walla Walla University & 1 & 1 & 1 & 1 & 1 & 5 \\
\hline 26. Washington Adventist University & 1 & 1 & 1 & & & 3 \\
\hline 27. Zaoksky Adventist University & & & $(1)^{*}$ & & $(1)^{*}$ & 0 \\
\hline Total number of respondents responded & 18 & 17 & 18 & 13 & 16 & 82 \\
\hline Total respondents included in the study & 18 & 17 & 16 & 13 & 14 & 78 \\
\hline
\end{tabular}

${ }^{*}$ Responded but not included in the study for not meeting the inclusion criteria. 
Table 7 gives a summary of the status groups and the number of institutions responded and included in the study by countries. The last two rows in the table include percentages of responses and cases included in the study. The response rate is $58.6 \%$.

Table 7. Summary of participating status groups by countries

\begin{tabular}{|c|c|c|c|c|c|c|c|}
\hline \multirow[b]{2}{*}{ Country } & \multirow[b]{2}{*}{$\begin{array}{l}\text { Number of } \\
\text { Institutions } \\
\text { Participated }\end{array}$} & \multicolumn{5}{|c|}{ Respondent Category } & \multirow{2}{*}{$\begin{array}{c}\text { Total Status } \\
\text { Groups } \\
\text { Included }\end{array}$} \\
\hline & & $\begin{array}{l}\text { Board } \\
\text { Chair }\end{array}$ & President & $\begin{array}{c}\text { Academic } \\
\text { VP }\end{array}$ & $\begin{array}{c}\text { Student } \\
\text { Service } \\
\text { VP }\end{array}$ & $\begin{array}{c}\text { Finance } \\
\text { VP }\end{array}$ & \\
\hline 1. Argentina & 1 & & & 1 & & $(1)^{*}$ & 1 \\
\hline 2. Australia & 1 & 1 & 1 & $(1)^{*}$ & & & 2 \\
\hline 3. Brazil & 1 & 1 & & & 1 & 1 & 3 \\
\hline 4. Canada & 1 & 1 & 1 & 1 & 1 & 1 & 5 \\
\hline 5. France & 1 & & 1 & & & & 1 \\
\hline 6. Germany & 1 & 1 & 1 & & & 1 & 3 \\
\hline 7. India & 1 & 1 & & 1 & 1 & & 3 \\
\hline 8. Kenya & 1 & & & 1 & 1 & & 2 \\
\hline 9. Korea & 1 & 1 & 1 & 1 & & 1 & 4 \\
\hline 10. Mexico & 1 & 1 & 1 & 1 & 1 & 1 & 5 \\
\hline 11. Papua New Guinea & 1 & & & 1 & 1 & & 2 \\
\hline 12. Philippines & 3 & 1 & 1 & 1 & & 2 & 5 \\
\hline 13. Puerto Rico & 1 & 1 & & & 1 & 1 & 3 \\
\hline 14. Russia & 1 & & & $(1)^{*}$ & & $(1)^{*}$ & 0 \\
\hline 15. U.S.A. & 10 & 8 & 10 & 8 & 6 & 6 & 38 \\
\hline 16. Zimbabwe & 1 & 1 & & & & & 1 \\
\hline Total responded & 27 & 18 & 17 & 18 & 13 & 16 & 82 \\
\hline Total included & 27 & 18 & 17 & 16 & 13 & 14 & 78 \\
\hline Percentages responded & & $66.7 \%$ & $63.0 \%$ & $66.7 \%$ & $48.1 \%$ & $59.3 \%$ & $58.6 \%$ \\
\hline $\begin{array}{l}\text { Percentages of respondents } \\
\text { included in the study }\end{array}$ & & $66.7 \%$ & $63.0 \%$ & $59.3 \%$ & $48.1 \%$ & $51.9 \%$ & $57.8 \%$ \\
\hline
\end{tabular}

${ }^{*}$ Responded but not included in the study for not meeting inclusion criteria. 


\section{Data Analysis}

Out of the 82 cases shown in Table 7, 78 cases were included in the study. These 78 respondents were comprised of 18 board chairs $(66.7 \%), 17$ presidents $(63.0 \%), 16$ academic vice presidents (59.3\%), 13 student service vice presidents (48.1\%), and 14 finance vice presidents (51.9\%) from 27 Seventh-day Adventist senior colleges and universities located in 16 countries.

Tables 8-14 present descriptive data of means $(M)$ and standard deviations $(S D)$ calculated from five status groups' responses to 47 questions listed under seven broad categories of functions. Table 15 presents a summary of means $(M)$ and standard deviations $(S D)$ of these functions by groups. The broad functions are as follows:

I. Student-Related Functions ( 8 items). See Table 8.

II. Employee-Related Functions (8 items). See Table 9.

III. General Administrative Functions (9 items). See Table 10.

IV. Finance-Related Functions (5 items). See Table 11.

V. Physical Facilities-Related Functions (6 items). See Table 12.

VI. Community- and Alumni-Related Functions (6 items). See Table 13.

VII. Spiritual Life-Related Functions (5 items). See Table 14.

A sample of 78 subjects consisted of board chairs, presidents, academic vice presidents, student service vice presidents, and finance vice presidents responded to the questions giving their perceptions on the extent of presidents' actual (A) role performances in 2004-2005, and their levels of expectations on presidents' expected (E) role responsibilities. 
Table 8. Means and standard deviations of actual and expected roles: I. Student-Related Functions

\begin{tabular}{|c|c|c|c|c|c|c|c|c|c|c|c|c|c|}
\hline \multirow{3}{*}{ Items } & & \multicolumn{10}{|c|}{ Respondent Category } & \multirow{2}{*}{\multicolumn{2}{|c|}{$\begin{array}{l}\text { Total } \\
n_{k}=78\end{array}$}} \\
\hline & & \multirow{2}{*}{\multicolumn{2}{|c|}{$\begin{array}{l}\text { Board Chair } \\
M^{n_{l}=18} S D\end{array}$}} & \multirow{2}{*}{\multicolumn{2}{|c|}{$\begin{array}{c}\text { President } \\
n_{2}=17\end{array}$}} & \multicolumn{2}{|c|}{$\begin{array}{c}\text { Academic } \\
\text { VP } \\
n_{3}=16\end{array}$} & \multicolumn{2}{|c|}{$\begin{array}{c}\text { Student } \\
\text { Service VP } \\
n_{4}=13\end{array}$} & \multicolumn{2}{|c|}{$\begin{array}{c}\text { Finance VP } \\
n_{5}=14\end{array}$} & & \\
\hline & & & & & & & $S D$ & & $S D$ & & $S D$ & $M$ & $S D$ \\
\hline 1. Participate in student recruitment & $\mathbf{A}$ & 3.50 & 1.15 & 3.65 & 1.17 & 2.63 & 1.15 & 3.46 & 1.45 & 3.36 & 1.34 & 3.32 & 1.26 \\
\hline$\sqrt{-1}+a^{2}$ & $\mathbf{E}$ & 4.11 & & 3.53 & 1.18 & 3.06 & 1.12 & 3.31 & 1.32 & 3.86 & .86 & 3.59 & 1.12 \\
\hline 2. Work closely with student & $\mathbf{A}$ & 3.61 & .92 & 3.59 & 1.23 & 3.06 & 1.18 & 3.15 & 1.46 & 2.93 & 1.38 & 3.29 & 1.23 \\
\hline government leaders & $\mathbf{E}$ & 4.06 & & 3.53 & 1.28 & 3.69 & 1.20 & 3.54 & 1.27 & 3.36 & 1.15 & 3.65 & 1.14 \\
\hline 3. Dialogue frequently with students & $\mathbf{A}$ & 3.72 & .96 & 4.24 & .75 & 3.25 & 1.29 & 3.54 & 1.20 & 3.86 & 1.35 & 3.73 & 1.14 \\
\hline & $\mathbf{E}$ & 4.56 & .51 & 4.00 & 1.12 & 3.94 & .85 & 4.23 & 1.01 & 4.21 & .70 & 4.19 & .87 \\
\hline 4. Visit students periodically in dorms & $\mathbf{A}$ & 2.78 & 1.17 & 2.88 & 1.32 & 2.13 & 1.26 & 2.15 & 1.07 & 2.71 & 1.33 & 2.55 & 1.24 \\
\hline during their dorm worships & $\mathbf{E}$ & 3.44 & 1.29 & 3.12 & 1.27 & 2.69 & 1.14 & 2.62 & 1.26 & 3.29 & 1.20 & 3.05 & 1.25 \\
\hline 5. Take ultimate responsibility & A & 3.50 & 1.29 & 3.00 & 1.58 & 2.19 & 1.11 & 3.00 & 1.68 & 2.86 & 1.41 & 2.92 & 1.45 \\
\hline for student discipline & $\mathbf{E}$ & 4.11 & 1.28 & 2.94 & 1.39 & 3.00 & 1.57 & 3.00 & 1.73 & 2.86 & 1.56 & 3.21 & 1.54 \\
\hline 6. Teach some classes to maintain & $\mathbf{A}$ & 2.56 & 1.69 & 2.94 & 1.56 & 1.75 & 1.24 & 2.00 & 1.29 & 2.93 & 1.69 & 2.45 & 1.55 \\
\hline contact with students & $\mathbf{E}$ & 3.00 & 1.68 & 2.88 & 1.58 & 2.31 & 1.20 & 1.46 & 1.61 & 2.93 & 1.54 & 2.73 & 1.52 \\
\hline 7. Know every student personally & $\mathbf{A}$ & 2.39 & 1.50 & 2.00 & .94 & 1.75 & .93 & 2.46 & 1.45 & 1.93 & 1.00 & 2.10 & 1.19 \\
\hline & $\mathbf{E}$ & 2.83 & 1.54 & 2.24 & 1.20 & 2.13 & 1.26 & 2.62 & 1.45 & 2.21 & 1.12 & 2.41 & 1.32 \\
\hline 8. Serve as mentor of students & $\mathbf{A}$ & 3.33 & 1.08 & 3.12 & 1.32 & 2.00 & .73 & 3.15 & 1.52 & 2.93 & 1.33 & 2.91 & 1.27 \\
\hline & $\mathbf{E}$ & 3.67 & 1.24 & 3.24 & 1.30 & 2.56 & 1.26 & 4.00 & 1.00 & 3.29 & 1.20 & 3.33 & 1.28 \\
\hline Total Average of Table 8 & $\mathbf{A}$ & 3.17 & 1.22 & 3.18 & 1.23 & 2.35 & 1.11 & 2.86 & 1.39 & 2.94 & 1.35 & 2.91 & 1.29 \\
\hline & $\mathbf{E}$ & 3.72 & 1.16 & 3.19 & 1.29 & 2.92 & 1.20 & 3.10 & 1.33 & 3.25 & 1.17 & 3.27 & 1.26 \\
\hline
\end{tabular}

Note: $\mathrm{A}=$ Actual $; \mathrm{E}=$ Expected 
Table 9. Means and standard deviations of actual and expected roles: II. Employee-Related Functions

\begin{tabular}{|c|c|c|c|c|c|c|c|c|c|c|c|c|c|}
\hline \multirow{3}{*}{ Items } & & \multicolumn{10}{|c|}{ Respondent Category } & \multirow{2}{*}{\multicolumn{2}{|c|}{$\begin{array}{l}\text { Total } \\
n_{k}=78\end{array}$}} \\
\hline & & \multicolumn{2}{|c|}{$\begin{array}{c}\text { Board Chair } \\
n_{l}=18\end{array}$} & \multicolumn{2}{|c|}{$\begin{array}{c}\text { President } \\
n_{2}=17\end{array}$} & \multicolumn{2}{|c|}{$\begin{array}{c}\text { Academic } \\
\text { VP } \\
n_{3}=16\end{array}$} & \multicolumn{2}{|c|}{$\begin{array}{c}\text { Student } \\
\text { Service VP } \\
n_{\boldsymbol{f}}=13\end{array}$} & \multicolumn{2}{|c|}{$\begin{array}{c}\text { Finance VP } \\
n_{5}=14\end{array}$} & & \\
\hline & & & & & & & & & & & & & $S D$ \\
\hline 9. Participate in faculty recruitment & A & 4.39 & .70 & 4.24 & .90 & 3.50 & .97 & 3.92 & 1.12 & 4.14 & 1.17 & 4.05 & .99 \\
\hline & & & & & & & & & & & 1.04 & 4.22 & .95 \\
\hline 10. Serve as academic leader of faculty & $\mathbf{A}$ & 3.22 & 1.48 & 3.12 & 1.27 & 2.56 & 1.03 & 2.69 & 1.38 & 3.21 & 1.05 & 2.97 & 1.26 \\
\hline & $\mathbf{E}$ & 3.67 & 1.41 & 3.24 & 1.20 & 3.00 & 1.21 & 3.31 & 1.38 & 3.29 & 1.14 & 3.31 & 1.26 \\
\hline 11. Guard faculty's academic freedom & $\mathbf{A}$ & 3.89 & & 4.06 & 1.20 & 3.31 & 1.14 & 3.31 & .95 & 3.93 & .92 & 3.72 & 1.04 \\
\hline & $\mathbf{E}$ & 4.33 & .77 & 4.24 & .97 & 4.06 & .85 & 3.85 & .90 & 4.00 & .88 & 4.12 & .87 \\
\hline $\begin{array}{l}\text { 12. Have responsibility in submitting } \\
\text { proposed changes in academic }\end{array}$ & $\mathbf{A}$ & 3.56 & 1.15 & 2.47 & 1.37 & 2.31 & 1.20 & 3.31 & 1.38 & 3.21 & 1.12 & 2.96 & 1.31 \\
\hline matters to faculty for approval & $\mathbf{E}$ & 3.78 & 1.22 & 2.65 & 1.37 & 2.56 & 1.26 & 3.62 & 1.33 & 3.50 & 1.40 & 3.21 & 1.38 \\
\hline 13. Work with faculty in reviewing & $\mathbf{A}$ & 4.06 & .87 & 4.00 & 1.06 & 2.94 & 1.29 & 3.85 & .80 & 3.79 & 1.37 & 3.73 & 1.15 \\
\hline accrediting agency's reports & $\mathbf{E}$ & 4.50 & .71 & 4.12 & 1.05 & 3.94 & 1.24 & 4.23 & .83 & 4.36 & 1.15 & 4.23 & 1.01 \\
\hline $\begin{array}{l}\text { 14. Recommend to board for hiring, } \\
\text { promotion, demotion, and }\end{array}$ & $\mathbf{A}$ & 3.83 & 1.25 & 4.29 & 1.26 & 4.06 & 1.34 & 4.46 & .97 & 4.21 & .70 & 4.15 & 1.14 \\
\hline dismissal of faculty and staff & $\mathbf{E}$ & 4.17 & 1.15 & 4.35 & 1.22 & 4.69 & .79 & 4.62 & .87 & 4.29 & .73 & 4.41 & .99 \\
\hline 15. Collaborate for advanced in-service & $\mathbf{A}$ & 3.67 & 1.14 & 3.82 & 1.01 & 2.63 & 1.45 & 3.85 & 1.21 & 3.14 & 1.10 & 3.42 & 1.25 \\
\hline training for faculty and staff & $\mathbf{E}$ & 4.11 & .96 & 3.65 & 1.11 & 3.00 & 1.46 & 4.08 & .95 & 3.43 & 1.34 & 3.65 & 1.23 \\
\hline 16. Carry out policies and regulations & $\mathbf{A}$ & 3.78 & 1.17 & 4.06 & 1.30 & 3.25 & 1.61 & 3.54 & 1.39 & 3.36 & 1.22 & 3.62 & 1.34 \\
\hline of faculty and staff & $\mathbf{E}$ & 4.11 & 1.08 & 4.06 & 1.34 & 3.75 & 1.53 & 3.92 & 1.19 & 3.57 & 1.22 & 3.90 & 1.26 \\
\hline Total Average of Table 9 & $\mathbf{A}$ & 3.80 & 1.07 & 3.76 & 1.17 & 3.07 & 1.25 & 3.62 & 1.15 & 3.62 & 1.08 & 3.58 & 1.19 \\
\hline & $\mathbf{E}$ & 4.17 & .97 & 3.81 & 1.13 & 3.63 & 1.18 & 3.96 & 1.08 & 3.81 & 1.11 & 3.88 & 1.12 \\
\hline
\end{tabular}

Note: $\mathrm{A}=$ Actual $; \mathrm{E}=$ Expected . 
Table 10. Means and standard deviations of actual and expected roles: III. General Administrative Functions

\begin{tabular}{|c|c|c|c|c|c|c|c|c|c|c|c|c|c|}
\hline \multirow[b]{2}{*}{ Items } & & \multicolumn{10}{|c|}{ Respondent Category } & \multirow{2}{*}{\multicolumn{2}{|c|}{$\begin{array}{c}\text { Total } \\
n_{k}=78\end{array}$}} \\
\hline & & \multicolumn{2}{|c|}{\begin{tabular}{|l} 
Board Chair \\
$n_{l}=18$ \\
$M \quad S D$ \\
\end{tabular}} & \multicolumn{2}{|c|}{$\begin{array}{c}\text { President } \\
n_{2}=17\end{array}$} & \multicolumn{2}{|c|}{$\begin{array}{c}\text { Academic } \\
\text { VP } \\
n_{3}=16\end{array}$} & \multicolumn{2}{|c|}{$\begin{array}{c}\text { Student } \\
\text { Service VP } \\
n_{t}=13\end{array}$} & \multicolumn{2}{|c|}{$\begin{array}{c}\text { Finance VP } \\
n_{5}=14\end{array}$} & & \\
\hline $\begin{array}{l}\text { 17. Prepare agenda for board of } \\
\text { trustees' meetings }\end{array}$ & $\begin{array}{l}\mathbf{A} \\
\mathbf{E}\end{array}$ & $\begin{array}{l}4.33 \\
4.72\end{array}$ & $\begin{array}{r}1.08 \\
.96\end{array}$ & $\begin{array}{l}5.00 \\
5.00\end{array}$ & $\begin{array}{l}.00 \\
.00\end{array}$ & $\begin{array}{l}4.75 \\
4.94\end{array}$ & $\begin{array}{l}.68 \\
.25\end{array}$ & $\begin{array}{l}4.54 \\
4.77\end{array}$ & $\begin{array}{l}.97 \\
.60\end{array}$ & $\begin{array}{l}4.57 \\
4.57\end{array}$ & $\begin{array}{l}.65 \\
.65\end{array}$ & $\begin{array}{l}4.64 \\
4.81\end{array}$ & $\begin{array}{l}.79 \\
.60\end{array}$ \\
\hline 18. Preside over general faculty meetings & $\begin{array}{l}\mathbf{A} \\
\mathbf{E}\end{array}$ & $\begin{array}{l}4.50 \\
4.61\end{array}$ & $\begin{array}{r}.86 \\
1.04\end{array}$ & $\begin{array}{l}4.12 \\
4.06\end{array}$ & $\begin{array}{l}1.36 \\
1.20\end{array}$ & $\begin{array}{l}4.06 \\
4.00\end{array}$ & $\begin{array}{l}1.29 \\
1.46\end{array}$ & $\begin{array}{l}3.46 \\
3.62\end{array}$ & $\begin{array}{l}1.51 \\
1.50\end{array}$ & $\begin{array}{l}3.57 \\
3.64\end{array}$ & $\begin{array}{l}1.50 \\
1.50\end{array}$ & $\begin{array}{l}3.99 \\
4.03\end{array}$ & $\begin{array}{l}1.32 \\
1.35\end{array}$ \\
\hline $\begin{array}{l}\text { 19. Take responsibility in structuring } \\
\text { student admission standards }\end{array}$ & $\begin{array}{c}\mathbf{A} \\
\mathbf{E}\end{array}$ & $\begin{array}{l}3.67 \\
4.06\end{array}$ & $\begin{array}{l}.77 \\
.87\end{array}$ & $\begin{array}{l}2.71 \\
2.71\end{array}$ & $\begin{array}{l}1.45 \\
1.31\end{array}$ & $\begin{array}{l}2.44 \\
3.06\end{array}$ & $\begin{array}{l}1.15 \\
1.24\end{array}$ & $\begin{array}{l}2.85 \\
3.15\end{array}$ & $\begin{array}{l}1.41 \\
1.41\end{array}$ & $\begin{array}{l}3.14 \\
3.29\end{array}$ & $\begin{array}{r}.86 \\
1.14\end{array}$ & $\begin{array}{l}2.97 \\
3.27\end{array}$ & $\begin{array}{l}1.21 \\
1.26\end{array}$ \\
\hline $\begin{array}{l}\text { 20. Take responsibility in strategic } \\
\text { planning for institution }\end{array}$ & $\begin{array}{l}\mathbf{A} \\
\mathbf{E}\end{array}$ & $\begin{array}{l}4.44 \\
4.83\end{array}$ & $\begin{array}{l}.86 \\
.38\end{array}$ & $\begin{array}{l}4.47 \\
4.59\end{array}$ & $\begin{array}{r}.80 \\
1.00\end{array}$ & $\begin{array}{l}3.94 \\
4.88\end{array}$ & $\begin{array}{r}1.39 \\
.34\end{array}$ & $\begin{array}{l}4.54 \\
4.69\end{array}$ & $\begin{array}{l}.97 \\
.63\end{array}$ & $\begin{array}{l}4.07 \\
4.79\end{array}$ & $\begin{array}{r}1.14 \\
.43\end{array}$ & $\begin{array}{l}4.29 \\
4.76\end{array}$ & $\begin{array}{r}1.05 \\
.61\end{array}$ \\
\hline $\begin{array}{l}\text { 21. Take a leading role to prepare, revise, } \\
\text { and follow organizational chart }\end{array}$ & $\mathbf{A}$ & $\begin{array}{l}4.28 \\
4.78\end{array}$ & $\begin{array}{l}.83 \\
.43\end{array}$ & $\begin{array}{l}4.35 \\
4.41\end{array}$ & $\begin{array}{l}1.11 \\
1.06\end{array}$ & $\begin{array}{l}3.50 \\
4.56\end{array}$ & $\begin{array}{r}1.37 \\
.73\end{array}$ & $\begin{array}{l}4.15 \\
4.54\end{array}$ & $\begin{array}{l}.99 \\
.66\end{array}$ & $\begin{array}{l}3.86 \\
4.29\end{array}$ & $\begin{array}{r}1.10 \\
.73\end{array}$ & $\begin{array}{l}4.04 \\
4.53\end{array}$ & $\begin{array}{r}1.11 \\
.75\end{array}$ \\
\hline $\begin{array}{l}\text { 22. Foster research and intellectual } \\
\text { climate for institution }\end{array}$ & $\begin{array}{l}\mathbf{A} \\
\mathbf{E}\end{array}$ & $\begin{array}{l}3.94 \\
4.17\end{array}$ & $\begin{array}{l}.94 \\
.92\end{array}$ & $\begin{array}{l}4.00 \\
4.18\end{array}$ & $\begin{array}{r}1.06 \\
.88\end{array}$ & $\begin{array}{l}3.38 \\
4.19\end{array}$ & $\begin{array}{r}1.31 \\
.91\end{array}$ & $\begin{array}{l}3.69 \\
4.54\end{array}$ & $\begin{array}{r}1.38 \\
.66\end{array}$ & $\begin{array}{l}4.00 \\
4.36\end{array}$ & $\begin{array}{l}.96 \\
.74\end{array}$ & $\begin{array}{l}3.80 \\
4.27\end{array}$ & $\begin{array}{r}1.13 \\
.83\end{array}$ \\
\hline $\begin{array}{l}\text { 23. Comply with legal mandates and } \\
\text { laws in addressing grievances }\end{array}$ & $\begin{array}{l}\mathbf{A} \\
\mathbf{E}\end{array}$ & $\begin{array}{l}4.33 \\
4.67\end{array}$ & $\begin{array}{l}.77 \\
.49\end{array}$ & $\begin{array}{l}4.59 \\
4.65\end{array}$ & $\begin{array}{l}1.00 \\
1.00\end{array}$ & $\begin{array}{l}4.31 \\
4.69\end{array}$ & $\begin{array}{l}.79 \\
.60\end{array}$ & $\begin{array}{l}4.15 \\
4.77\end{array}$ & $\begin{array}{l}.99 \\
.44\end{array}$ & $\begin{array}{l}3.79 \\
4.21\end{array}$ & $\begin{array}{r}1.05 \\
.97\end{array}$ & $\begin{array}{l}4.26 \\
4.60\end{array}$ & $\begin{array}{l}.93 \\
.74\end{array}$ \\
\hline $\begin{array}{l}\text { 24. Help formulate and adhere to } \\
\text { educational philosophy }\end{array}$ & $\mathbf{A}$ & $\begin{array}{l}4.61 \\
5.00\end{array}$ & $\begin{array}{l}.61 \\
.00\end{array}$ & $\begin{array}{l}4.65 \\
4.76\end{array}$ & $\begin{array}{l}.86 \\
.56\end{array}$ & $\begin{array}{l}3.75 \\
4.88\end{array}$ & $\begin{array}{r}1.39 \\
.34\end{array}$ & $\begin{array}{l}4.69 \\
4.92\end{array}$ & $\begin{array}{l}.48 \\
.28\end{array}$ & $\begin{array}{l}4.50 \\
4.79\end{array}$ & $\begin{array}{l}.65 \\
.43\end{array}$ & $\begin{array}{l}4.44 \\
4.87\end{array}$ & $\begin{array}{l}.92 \\
.37\end{array}$ \\
\hline $\begin{array}{l}\text { 25. Delegate responsibility, authority, and } \\
\text { needed resources to appropriate } \\
\text { administrators and support their roles }\end{array}$ & $\begin{array}{l}\mathbf{A} \\
\mathbf{E}\end{array}$ & $\begin{array}{l}3.89 \\
4.44\end{array}$ & $\begin{array}{r}1.02 \\
.70\end{array}$ & $\begin{array}{l}4.71 \\
4.76\end{array}$ & $\begin{array}{l}.77 \\
.44\end{array}$ & $\begin{array}{l}4.13 \\
4.94\end{array}$ & $\begin{array}{r}1.15 \\
.25\end{array}$ & $\begin{array}{l}3.85 \\
4.85\end{array}$ & $\begin{array}{r}1.46 \\
.38\end{array}$ & $\begin{array}{l}4.07 \\
4.71\end{array}$ & $\begin{array}{r}1.21 \\
.61\end{array}$ & $\begin{array}{l}4.14 \\
4.73\end{array}$ & $\begin{array}{r}1.14 \\
.53\end{array}$ \\
\hline Total Average of Table 10 & $\begin{array}{l}\mathbf{A} \\
\mathbf{E}\end{array}$ & $\begin{array}{l}4.22 \\
4.59\end{array}$ & $\begin{array}{l}.86 \\
.64\end{array}$ & $\begin{array}{l}4.29 \\
4.35\end{array}$ & $\begin{array}{l}.93 \\
.83\end{array}$ & $\begin{array}{l}3.81 \\
4.46\end{array}$ & $\begin{array}{r}1.17 \\
.68\end{array}$ & $\begin{array}{l}3.99 \\
4.43\end{array}$ & $\begin{array}{r}1.13 \\
.73\end{array}$ & $\begin{array}{l}3.95 \\
4.29\end{array}$ & $\begin{array}{r}1.01 \\
.80\end{array}$ & $\begin{array}{l}4.06 \\
4.43\end{array}$ & $\begin{array}{r}1.07 \\
.78\end{array}$ \\
\hline
\end{tabular}

Note: $\mathrm{A}=$ Actual; $\mathrm{E}=$ Expected. 
Table 11. Means and standard deviations of actual and expected roles: IV. Finance-Related Functions

\begin{tabular}{|c|c|c|c|c|c|c|c|c|c|c|c|c|c|}
\hline \multirow{3}{*}{ Items } & & \multicolumn{10}{|c|}{ Respondent Category } & \multirow{2}{*}{\multicolumn{2}{|c|}{$\begin{array}{l}\text { Total } \\
n_{k}=78\end{array}$}} \\
\hline & & \multirow{2}{*}{\multicolumn{2}{|c|}{$\begin{array}{l}\text { Board Chair } \\
M^{n_{l}=18} S D\end{array}$}} & \multicolumn{2}{|c|}{$\begin{array}{c}\text { President } \\
n_{2}=17\end{array}$} & \multicolumn{2}{|c|}{$\begin{array}{c}\text { Academic } \\
\text { VP } \\
n_{3}=16\end{array}$} & \multicolumn{2}{|c|}{$\begin{array}{c}\text { Student } \\
\text { Service VP } \\
n_{t}=13\end{array}$} & \multicolumn{2}{|c|}{$\begin{array}{c}\text { Finance VP } \\
n_{5}=14\end{array}$} & & \\
\hline & & & & & & & & & & & & $M$ & $S D$ \\
\hline $\begin{array}{l}\text { 26. Participate in the preparation of } \\
\text { annual operating budget of institution }\end{array}$ & $\begin{array}{l}\mathbf{A} \\
\mathbf{E}\end{array}$ & $\begin{array}{l}4.00 \\
4.72\end{array}$ & $\begin{array}{r}1.08 \\
.46\end{array}$ & $\begin{array}{l}4.24 \\
4.47\end{array}$ & $\begin{array}{l}1.35 \\
1.23\end{array}$ & $\begin{array}{l}3.56 \\
4.50\end{array}$ & $\begin{array}{r}1.36 \\
.63\end{array}$ & $\begin{array}{l}4.31 \\
4.54\end{array}$ & $\begin{array}{r}1.18 \\
.66\end{array}$ & $\begin{array}{l}3.50 \\
3.64\end{array}$ & $\begin{array}{l}1.40 \\
1.08\end{array}$ & $\begin{array}{l}3.92 \\
4.40\end{array}$ & $\begin{array}{r}1.29 \\
.92\end{array}$ \\
\hline $\begin{array}{l}\text { 27. Ensure that institution's operating } \\
\text { budget, after approval by board, } \\
\text { is adhered to }\end{array}$ & $\begin{array}{l}\mathbf{A} \\
\mathbf{E}\end{array}$ & $\begin{array}{l}3.61 \\
4.67\end{array}$ & $\begin{array}{r}1.20 \\
.49\end{array}$ & $\begin{array}{l}4.00 \\
4.24\end{array}$ & $\begin{array}{l}1.32 \\
1.30\end{array}$ & $\begin{array}{l}3.75 \\
4.69\end{array}$ & $\begin{array}{r}1.06 \\
.60\end{array}$ & $\begin{array}{l}3.77 \\
4.38\end{array}$ & $\begin{array}{r}1.24 \\
.96\end{array}$ & $\begin{array}{l}3.50 \\
4.21\end{array}$ & $\begin{array}{r}1.29 \\
.80\end{array}$ & $\begin{array}{l}3.73 \\
4.45\end{array}$ & $\begin{array}{r}1.20 \\
.88\end{array}$ \\
\hline $\begin{array}{l}\text { 28. Present annual audited financial } \\
\text { statements to board }\end{array}$ & $\begin{array}{l}\mathbf{A} \\
\mathbf{E}\end{array}$ & $\begin{array}{l}3.17 \\
3.61\end{array}$ & $\begin{array}{l}1.34 \\
1.50\end{array}$ & $\begin{array}{l}3.18 \\
3.18\end{array}$ & $\begin{array}{l}1.59 \\
1.59\end{array}$ & $\begin{array}{l}3.25 \\
3.81\end{array}$ & $\begin{array}{l}1.53 \\
1.33\end{array}$ & $\begin{array}{l}3.31 \\
3.46\end{array}$ & $\begin{array}{l}1.44 \\
1.45\end{array}$ & $\begin{array}{l}2.64 \\
2.79\end{array}$ & $\begin{array}{l}1.55 \\
1.63\end{array}$ & $\begin{array}{l}3.12 \\
3.38\end{array}$ & $\begin{array}{l}1.47 \\
1.51\end{array}$ \\
\hline $\begin{array}{l}\text { 29. Recommend to board the salary } \\
\text { changes for faculty/staff positions }\end{array}$ & $\begin{array}{l}\mathbf{A} \\
\mathbf{E}\end{array}$ & $\begin{array}{l}3.06 \\
3.22\end{array}$ & $\begin{array}{l}1.35 \\
1.40\end{array}$ & $\begin{array}{l}3.88 \\
3.94\end{array}$ & $\begin{array}{l}1.58 \\
1.52\end{array}$ & $\begin{array}{l}3.38 \\
4.06\end{array}$ & $\begin{array}{l}1.45 \\
1.18\end{array}$ & $\begin{array}{l}3.31 \\
4.00\end{array}$ & $\begin{array}{l}1.18 \\
1.22\end{array}$ & $\begin{array}{l}2.71 \\
3.29\end{array}$ & $\begin{array}{l}1.38 \\
1.54\end{array}$ & $\begin{array}{l}3.28 \\
3.69\end{array}$ & $\begin{array}{l}1.42 \\
1.40\end{array}$ \\
\hline $\begin{array}{l}\text { 30. Accept accountability to board } \\
\text { for school's financial operation }\end{array}$ & $\begin{array}{l}\mathbf{A} \\
\mathbf{E}\end{array}$ & $\begin{array}{l}4.11 \\
4.78\end{array}$ & $\begin{array}{r}1.13 \\
.43\end{array}$ & $\begin{array}{l}4.35 \\
4.47\end{array}$ & $\begin{array}{l}1.17 \\
1.12\end{array}$ & $\begin{array}{l}4.12 \\
4.81\end{array}$ & $\begin{array}{l}.89 \\
.54\end{array}$ & $\begin{array}{l}3.62 \\
4.38\end{array}$ & $\begin{array}{l}1.26 \\
1.12\end{array}$ & $\begin{array}{l}3.93 \\
4.57\end{array}$ & $\begin{array}{r}1.27 \\
.85\end{array}$ & $\begin{array}{l}4.05 \\
4.62\end{array}$ & $\begin{array}{r}1.14 \\
.84\end{array}$ \\
\hline Total Average of Table 11 & $\begin{array}{l}\mathbf{A} \\
\mathbf{E}\end{array}$ & $\begin{array}{l}3.59 \\
4.20\end{array}$ & $\begin{array}{r}1.22 \\
.86\end{array}$ & $\begin{array}{l}3.93 \\
4.06\end{array}$ & $\begin{array}{l}1.40 \\
1.35\end{array}$ & $\begin{array}{l}3.61 \\
4.37\end{array}$ & $\begin{array}{r}1.26 \\
.86\end{array}$ & $\begin{array}{l}3.66 \\
4.15\end{array}$ & $\begin{array}{l}1.26 \\
1.08\end{array}$ & $\begin{array}{l}3.26 \\
3.70\end{array}$ & $\begin{array}{l}1.38 \\
1.18\end{array}$ & $\begin{array}{l}3.62 \\
4.11\end{array}$ & $\begin{array}{l}1.30 \\
1.11\end{array}$ \\
\hline
\end{tabular}

Note: $\mathrm{A}=$ Actual; $\mathrm{E}=$ Expected. 
Table 12. Means and standard deviations of actual and expected roles: V. Physical Facilities-Related Functions

\begin{tabular}{|c|c|c|c|c|c|c|c|c|c|c|c|c|c|}
\hline \multirow{3}{*}{ Items } & & \multicolumn{10}{|c|}{ Respondent Category } & \multirow{2}{*}{\multicolumn{2}{|c|}{$\begin{array}{l}\text { Total } \\
n_{k}=78\end{array}$}} \\
\hline & & \multirow{2}{*}{\multicolumn{2}{|c|}{$\begin{array}{l}\text { Board Chair } \\
n_{I}=18 \\
\quad S D\end{array}$}} & \multicolumn{2}{|c|}{$\begin{array}{c}\text { President } \\
n_{2}=17\end{array}$} & \multicolumn{2}{|c|}{$\begin{array}{c}\text { Academic } \\
\text { VP } \\
n_{3}=16\end{array}$} & \multicolumn{2}{|c|}{$\begin{array}{c}\text { Student } \\
\text { Service VP } \\
n_{\mathfrak{f}}=13\end{array}$} & \multicolumn{2}{|c|}{$\begin{array}{c}\text { Finance VP } \\
n_{5}=14\end{array}$} & & \\
\hline & & & & & $S D$ & & & & & & $S D$ & $M$ & $S D$ \\
\hline $\begin{array}{l}\text { 31. Present to board the physical } \\
\text { needs of institution }\end{array}$ & $\begin{array}{l}\mathbf{A} \\
\mathbf{E}\end{array}$ & $\begin{array}{l}4.44 \\
4.61\end{array}$ & $\begin{array}{l}.70 \\
.61\end{array}$ & $\begin{array}{l}4.29 \\
4.29\end{array}$ & $\begin{array}{l}1.10 \\
1.10\end{array}$ & $\begin{array}{l}4.00 \\
4.69\end{array}$ & $\begin{array}{r}1.32 \\
1.01\end{array}$ & $\begin{array}{l}4.23 \\
4.54\end{array}$ & $\begin{array}{l}1.17 \\
1.13\end{array}$ & $\begin{array}{l}3.21 \\
3.43\end{array}$ & $\begin{array}{l}1.63 \\
1.55\end{array}$ & $\begin{array}{l}4.06 \\
4.33\end{array}$ & $\begin{array}{l}1.24 \\
1.16\end{array}$ \\
\hline 32. Direct the preparation of plans for new & $\mathbf{A}$ & 4.06 & 1.00 & 3.35 & 1.54 & 3.69 & 1.35 & 3.46 & 1.66 & 2.79 & 1.53 & 3.50 & 1.44 \\
\hline buildings and installations as needed & & 4.11 & .90 & & 1.28 & 4.31 & .95 & 4.00 & 1.15 & 3.07 & 1.49 & 3.79 & 1.22 \\
\hline 33. Plan for major repairs and alterations & $\mathbf{A}$ & 3.44 & 1.25 & 2.65 & 1.46 & 3.25 & 1.29 & 3.23 & 1.64 & 2.64 & 1.28 & 3.05 & 1.39 \\
\hline of plant, buildings, and equipment & $\mathbf{E}$ & 3.50 & 1.20 & 2.65 & 1.46 & 3.44 & 1.26 & 3.54 & 1.39 & 2.71 & 1.27 & 3.17 & 1.34 \\
\hline 34. Recommend to board for equipment & $\mathbf{A}$ & 3.50 & 1.42 & 3.00 & 1.58 & 3.00 & 1.59 & 3.15 & 1.52 & 2.50 & 1.40 & 3.05 & 1.50 \\
\hline acquisition & $\mathbf{E}$ & 3.67 & 1.64 & 3.12 & 1.65 & 3.75 & 1.53 & 3.38 & 1.45 & 2.79 & 1.37 & 3.36 & 1.55 \\
\hline $\begin{array}{l}\text { 35. Ensure that physical plant master } \\
\text { plans are current and followed as }\end{array}$ & A & 4.06 & 1.63 & 3.94 & 1.30 & 3.63 & 1.26 & 3.23 & 1.30 & 3.07 & 1.07 & 3.63 & 1.36 \\
\hline closely as possible & $\mathbf{E}$ & 4.28 & 1.07 & 4.12 & 1.22 & 4.19 & 1.22 & 4.15 & .80 & 3.64 & 1.01 & 4.09 & 1.08 \\
\hline $\begin{array}{l}\text { 36. Ascertain that campus grounds are } \\
\text { well-kent through anpropriate }\end{array}$ & A & 3.78 & 1.22 & 4.18 & 1.13 & 3.56 & 1.31 & 3.69 & 1.11 & 3.36 & 1.08 & 3.73 & 1.18 \\
\hline officer and administrator & $\mathbf{E}$ & 4.11 & 1.13 & 4.12 & 1.22 & 4.31 & .95 & 3.85 & 1.14 & 3.71 & 1.20 & 4.04 & 1.12 \\
\hline Total Average of Table 12 & $\mathbf{A}$ & 3.88 & 1.20 & 3.57 & 1.35 & 3.52 & 1.35 & 3.50 & 1.40 & 2.93 & 1.33 & 3.50 & 1.35 \\
\hline & $\mathbf{E}$ & 4.05 & 1.09 & 4.34 & 1.32 & 4.12 & 1.15 & 3.91 & 1.18 & 3.23 & 1.32 & 3.80 & 1.25 \\
\hline
\end{tabular}

Note: $\mathrm{A}=$ Actual; $\mathrm{E}=$ Expected. 
Table 13. Means and standard deviations of actual and expected roles: VI. Community- and Alumni-Related Functions

\begin{tabular}{|c|c|c|c|c|c|c|c|c|c|c|c|c|c|}
\hline \multirow{3}{*}{ Items } & & \multicolumn{10}{|c|}{ Respondent Category } & \multirow{2}{*}{\multicolumn{2}{|c|}{$\begin{array}{c}\text { Total } \\
n_{k}=78\end{array}$}} \\
\hline & & \multicolumn{2}{|c|}{$\begin{array}{c}\text { Board Chair } \\
n_{t}=18\end{array}$} & \multicolumn{2}{|c|}{$\begin{array}{c}\text { President } \\
n_{2}=17\end{array}$} & \multicolumn{2}{|c|}{$\begin{array}{c}\text { Academic } \\
\text { VP } \\
n_{3}=16\end{array}$} & \multicolumn{2}{|c|}{$\begin{array}{c}\text { Student } \\
\text { Service VP } \\
n_{J}=13\end{array}$} & \multicolumn{2}{|c|}{$\begin{array}{c}\text { Finance VP } \\
n_{5}=14\end{array}$} & & \\
\hline & & $M$ & & $M$ & & $M$ & & $M$ & & & $S D$ & $M$ & $S D$ \\
\hline 37. Assist in raising funds for & $\mathbf{A}$ & 4.06 & 1.21 & 4.23 & 1.25 & 3.94 & 1.29 & 4.31 & 1.11 & 3.93 & .92 & 4.09 & 1.15 \\
\hline support oi institution & & & & & & 4.94 & .25 & & .83 & 4.11 & & & .04 \\
\hline 38. Represent institution to churches & $\mathbf{A}$ & 4.28 & .75 & 4.88 & .33 & 3.94 & 1.34 & 4.69 & .85 & 4.21 & .80 & 4.40 & .92 \\
\hline of the constituency & $\mathbf{E}$ & 4.89 & .32 & 4.94 & .24 & 4.69 & .48 & 5.00 & .00 & 4.71 & .61 & 4.85 & .40 \\
\hline 39. Present reports about institution & $\mathbf{A}$ & 4.61 & .50 & 4.76 & .66 & 4.06 & 1.18 & 4.31 & 1.32 & 3.50 & 1.22 & 4.28 & 1.07 \\
\hline to community and alumni & $\mathbf{E}$ & 4.78 & .43 & 4.82 & .53 & 4.63 & .62 & 4.85 & .38 & 4.21 & .97 & 4.67 & .64 \\
\hline 40. Represent institution as its official & $\mathbf{A}$ & 4.39 & 1.20 & 4.76 & .75 & 4.44 & .73 & 4.77 & .60 & 4.07 & .83 & 4.49 & .88 \\
\hline spokesperson to public & $\mathbf{E}$ & 4.72 & & 4.76 & .75 & 4.88 & .34 & 4.92 & .28 & 4.50 & .85 & 4.76 & .71 \\
\hline 41. Associate closely with alumni & $\mathbf{A}$ & 3.83 & 1.04 & 4.35 & .86 & 4.19 & .83 & 4.15 & 1.28 & 3.71 & .99 & 4.05 & 1.01 \\
\hline groups & $\mathbf{E}$ & 4.56 & .62 & 4.53 & .62 & 4.56 & .63 & 4.46 & .66 & 4.43 & .65 & 4.51 & .62 \\
\hline 42. Listen to constituency and alumni & $\mathbf{A}$ & 4.06 & 1.26 & 4.76 & .44 & 3.94 & .93 & 4.31 & 1.38 & 4.07 & .92 & 4.23 & 1.04 \\
\hline & $\mathbf{E}$ & 4.78 & .43 & 4.94 & .24 & 4.63 & .50 & 4.85 & .38 & 4.50 & .65 & 4.74 & .47 \\
\hline Total Average of Table 13 & $\mathbf{A}$ & 4.21 & .99 & 4.62 & .72 & 4.09 & 1.05 & 4.42 & 1.09 & 3.92 & .95 & 4.26 & 1.01 \\
\hline & $\mathbf{E}$ & 4.75 & .53 & 4.77 & .56 & 4.72 & .47 & 4.81 & .42 & 4.51 & .70 & 4.72 & .58 \\
\hline
\end{tabular}

Note: $\mathrm{A}=$ Actual; $\mathrm{E}=$ Expected. 
Table 14. Means and standard deviations of actual and expected roles: VII. Spiritual Life-Related Functions

\begin{tabular}{|c|c|c|c|c|c|c|c|c|c|c|c|c|c|}
\hline \multirow{2}{*}{\multicolumn{2}{|c|}{ Items }} & \multicolumn{10}{|c|}{ Respondent Category } & \multirow{2}{*}{\multicolumn{2}{|c|}{$\begin{array}{l}\text { Total } \\
n_{k}=78 \\
S D\end{array}$}} \\
\hline & & \multicolumn{2}{|c|}{$\begin{array}{l}\text { Board Chair } \\
M_{l}=18 \\
n_{1}\end{array}$} & \multicolumn{2}{|c|}{$\begin{array}{c}\text { President } \\
n_{2}=17\end{array}$} & \multicolumn{2}{|c|}{$\begin{array}{c}\text { Academic } \\
\text { VP } \\
n_{3}=16\end{array}$} & \multicolumn{2}{|c|}{$\begin{array}{l}\text { Student } \\
\text { Service VP } \\
n_{f}=13\end{array}$} & \multicolumn{2}{|c|}{$\begin{array}{c}\text { Finance VP } \\
n_{5}=14\end{array}$} & & \\
\hline $\begin{array}{l}\text { 43. Assume primary responsibility for } \\
\text { promotion of religious life of students } \\
\text { at the institution via delegation }\end{array}$ & $\begin{array}{l}\mathbf{A} \\
\mathbf{E}\end{array}$ & $\begin{array}{l}3.94 \\
4.83\end{array}$ & $\begin{array}{r}1.16 \\
.51\end{array}$ & $\begin{array}{l}4.71 \\
4.71\end{array}$ & $\begin{array}{l}.69 \\
.59\end{array}$ & $\begin{array}{l}3.81 \\
4.38\end{array}$ & $\begin{array}{l}.98 \\
.72\end{array}$ & $\begin{array}{l}3.69 \\
3.85\end{array}$ & $\begin{array}{l}1.44 \\
1.68\end{array}$ & $\begin{array}{l}4.14 \\
4.43\end{array}$ & $\begin{array}{r}1.03 \\
.65\end{array}$ & $\begin{array}{l}4.08 \\
4.47\end{array}$ & $\begin{array}{r}1.10 \\
.92\end{array}$ \\
\hline $\begin{array}{l}\text { 44. Assume primary responsibility for } \\
\text { promotion of religious life of faculty } \\
\text { and staff at the institution via delegation }\end{array}$ & $\begin{array}{l}\text { A } \\
\text { E }\end{array}$ & $\begin{array}{l}3.78 \\
4.72\end{array}$ & $\begin{array}{r}1.11 \\
.57\end{array}$ & $\begin{array}{l}4.29 \\
4.41\end{array}$ & $\begin{array}{l}1.26 \\
1.12\end{array}$ & $\begin{array}{l}3.25 \\
4.00\end{array}$ & $\begin{array}{l}1.13 \\
1.03\end{array}$ & $\begin{array}{l}3.69 \\
4.31\end{array}$ & $\begin{array}{l}1.18 \\
1.32\end{array}$ & $\begin{array}{l}3.79 \\
4.21\end{array}$ & $\begin{array}{r}1.19 \\
.80\end{array}$ & $\begin{array}{l}3.77 \\
4.35\end{array}$ & $\begin{array}{r}1.19 \\
.99\end{array}$ \\
\hline $\begin{array}{l}\text { 45. Take ultimate responsibility for planning } \\
\text { midweek chapels, vespers, weeks of } \\
\text { spiritual emphasis and other religious } \\
\text { programs on campus for spiritual nurture }\end{array}$ & $\begin{array}{l}\mathbf{A} \\
\mathbf{E}\end{array}$ & $\begin{array}{l}3.44 \\
4.39\end{array}$ & $\begin{array}{l}1.34 \\
1.09\end{array}$ & $\begin{array}{l}3.71 \\
3.82\end{array}$ & $\begin{array}{l}1.40 \\
1.33\end{array}$ & $\begin{array}{l}3.13 \\
3.81\end{array}$ & $\begin{array}{r}1.20 \\
.91\end{array}$ & $\begin{array}{l}2.85 \\
3.46\end{array}$ & $\begin{array}{l}1.46 \\
1.71\end{array}$ & $\begin{array}{l}3.50 \\
3.93\end{array}$ & $\begin{array}{l}1.29 \\
1.00\end{array}$ & $\begin{array}{l}3.35 \\
3.91\end{array}$ & $\begin{array}{l}1.34 \\
1.23\end{array}$ \\
\hline $\begin{array}{l}\text { 46. Manifest Christ-like character } \\
\text { in spiritual leadership roles }\end{array}$ & $\begin{array}{l}\mathbf{A} \\
\mathbf{E}\end{array}$ & $\begin{array}{l}4.50 \\
4.94\end{array}$ & $\begin{array}{l}.71 \\
.24\end{array}$ & $\begin{array}{l}4.82 \\
4.82\end{array}$ & $\begin{array}{l}.53 \\
.53\end{array}$ & $\begin{array}{l}4.13 \\
4.88\end{array}$ & $\begin{array}{l}.72 \\
.34\end{array}$ & $\begin{array}{l}4.46 \\
4.85\end{array}$ & $\begin{array}{l}.97 \\
.38\end{array}$ & $\begin{array}{l}4.36 \\
4.64\end{array}$ & $\begin{array}{r}1.01 \\
.63\end{array}$ & $\begin{array}{l}4.46 \\
4.83\end{array}$ & $\begin{array}{l}.80 \\
.44\end{array}$ \\
\hline $\begin{array}{l}\text { 47. Focus on Christian philosophy of } \\
\text { institution }\end{array}$ & $\begin{array}{l}\mathbf{A} \\
\mathbf{E}\end{array}$ & $\begin{array}{l}4.72 \\
4.94\end{array}$ & $\begin{array}{l}.57 \\
.24\end{array}$ & $\begin{array}{l}4.94 \\
4.94\end{array}$ & $\begin{array}{l}.24 \\
.24\end{array}$ & $\begin{array}{l}4.56 \\
4.88\end{array}$ & $\begin{array}{l}.73 \\
.34\end{array}$ & $\begin{array}{l}4.54 \\
4.92\end{array}$ & $\begin{array}{r}1.20 \\
.28\end{array}$ & $\begin{array}{l}4.36 \\
4.71\end{array}$ & $\begin{array}{l}.93 \\
.47\end{array}$ & $\begin{array}{l}4.64 \\
4.88\end{array}$ & $\begin{array}{l}.77 \\
.32\end{array}$ \\
\hline Total Average of Table 14 & $\begin{array}{l}\mathbf{A} \\
\mathbf{E}\end{array}$ & $\begin{array}{l}4.08 \\
4.76\end{array}$ & $\begin{array}{l}.98 \\
.53\end{array}$ & $\begin{array}{l}4.49 \\
4.54\end{array}$ & $\begin{array}{l}.82 \\
.76\end{array}$ & $\begin{array}{l}3.78 \\
4.39\end{array}$ & $\begin{array}{l}.95 \\
.67\end{array}$ & $\begin{array}{l}3.85 \\
4.28\end{array}$ & $\begin{array}{l}1.25 \\
1.07\end{array}$ & $\begin{array}{l}4.03 \\
4.38\end{array}$ & $\begin{array}{r}1.09 \\
.71\end{array}$ & $\begin{array}{l}4.06 \\
4.49\end{array}$ & $\begin{array}{r}1.04 \\
.78\end{array}$ \\
\hline Grand Total Average of Tables 8-14 & $\begin{array}{l}\mathbf{A} \\
\mathbf{E}\end{array}$ & $\begin{array}{l}3.85 \\
4.32\end{array}$ & $\begin{array}{r}1.08 \\
.83\end{array}$ & $\begin{array}{l}3.98 \\
4.15\end{array}$ & $\begin{array}{l}1.09 \\
1.03\end{array}$ & $\begin{array}{l}3.46 \\
4.09\end{array}$ & $\begin{array}{r}1.16 \\
.89\end{array}$ & $\begin{array}{l}3.70 \\
4.09\end{array}$ & $\begin{array}{r}1.24 \\
.98\end{array}$ & $\begin{array}{l}3.52 \\
3.88\end{array}$ & $\begin{array}{l}1.17 \\
1.00\end{array}$ & $\begin{array}{l}3.70 \\
4.11\end{array}$ & $\begin{array}{r}1.15 \\
.95\end{array}$ \\
\hline
\end{tabular}

Note: $\mathrm{A}=$ Actual; $\mathrm{E}=$ Expected. 
Table 15. Summary of means and standard deviations of actual and expected roles (Tables 8-14)

\begin{tabular}{|c|c|c|c|c|c|c|c|c|c|c|c|c|c|}
\hline \multirow[t]{2}{*}{ Seven Broad Categories of Functions } & & \multicolumn{2}{|c|}{$\begin{array}{l}\text { Board } \\
\text { Chair } \\
n_{1}=18\end{array}$} & \multicolumn{2}{|c|}{$\begin{array}{l}\text { President } \\
n_{2}=17\end{array}$} & \multicolumn{2}{|c|}{$\begin{array}{c}\text { Academic } \\
\text { VP } \\
n_{3}=16\end{array}$} & \multicolumn{2}{|c|}{$\begin{array}{c}\text { Student } \\
\text { Service VP } \\
n_{f}=13\end{array}$} & \multicolumn{2}{|c|}{$\begin{array}{l}\text { Finance VP } \\
\qquad n_{5}=14\end{array}$} & \multicolumn{2}{|c|}{$\begin{array}{c}\text { Groups' Total } \\
\text { by Items } \\
n_{k}=78\end{array}$} \\
\hline & & $M$ & $S D$ & $M$ & $S D$ & $M$ & $S D$ & & $S D$ & & $S D$ & & \\
\hline \multirow[t]{2}{*}{ I. Student-Related Functions (8 items) } & $\mathbf{A}$ & 3.17 & 1.22 & 3.18 & 1.23 & 2.35 & 1.11 & 2.86 & 1.39 & 2.94 & 1.35 & 2.91 & 1.29 \\
\hline & $\mathbf{E}$ & 3.72 & 1.16 & 3.19 & 1.29 & 2.92 & 1.20 & 3.10 & 1.33 & 3.25 & 1.17 & 3.27 & 1.26 \\
\hline \multirow[t]{2}{*}{ II. Employee-Related Functions (8 items) } & $\mathbf{A}$ & 3.80 & 1.07 & 3.76 & 1.17 & 3.07 & 1.25 & 3.62 & 1.15 & 3.62 & 1.08 & 3.58 & 1.19 \\
\hline & $\mathbf{E}$ & 4.17 & .97 & 3.81 & 1.13 & 3.63 & 1.18 & 3.96 & 1.08 & 3.81 & 1.11 & 3.88 & 1.12 \\
\hline \multirow[t]{2}{*}{ III. General Administrative Functions ( 9 items) } & $\mathbf{A}$ & 4.22 & & $4: 29$ & .93 & 3.81 & 1.17 & 3.99 & 1.13 & 3.95 & 1.01 & 4.06 & 1.07 . \\
\hline & $\mathbf{E}$ & 4.59 & .64 & 4.35 & .83 & 4.46 & .68 & 4.43 & .73 & 4.29 & .80 & 4.43 & .78 \\
\hline \multirow[t]{2}{*}{ IV. Finance-Related Functions (5 items) } & $\mathbf{A}$ & 3.59 & 1.22 & 3.93 & 1.40 & 3.61 & 1.26 & 3.66 & 1.26 & 3.26 & 1.38 & 3.62 & 1.30 \\
\hline & $\mathbf{E}$ & 4.20 & .86 & 4.06 & 1.35 & 4.37 & .86 & 4.15 & 1.08 & 3.70 & 1.18 & 4.11 & 1.11 \\
\hline \multirow[t]{2}{*}{ V. Physical Facilities-Related Functions (6 items) } & $\mathbf{A}$ & 3.88 & 1.20 & 3.57 & 1.35 & 3.52 & 1.35 & 3.50 & 1.40 & 2.93 & 1.33 & 3.50 & 1.35 \\
\hline & $\mathbf{E}$ & 4.05 & 1.09 & 4.34 & 1.32 & 4.12 & 1.15 & 3.91 & 1.18 & 3.23 & 1.32 & 3.80 & 1.25 \\
\hline \multirow{2}{*}{$\begin{array}{l}\text { VI. Community- and Alumni-Related Functions } \\
\text { (6 items) }\end{array}$} & $\mathbf{A}$ & 4.21 & .99 & 4.62 & .72 & 4.09 & 1.05 & 4.42 & 1.09 & 3.92 & .95 & 4.26 & 1.01 \\
\hline & $\mathbf{E}$ & 4.75 & .53 & 4.77 & .56 & 4.72 & .47 & 4.81 & .42 & 4.51 & .70 & 4.72 & .58 \\
\hline \multirow[t]{2}{*}{ VII. Spiritual Life-Related Functions (5 items) } & $\mathbf{A}$ & 4.08 & .98 & 4.49 & .82 & 3.78 & .95 & 3.85 & 1.25 & 4.03 & 1.09 & 4.06 & 1.04 \\
\hline & $\mathbf{E}$ & 4.76 & .53 & 4.54 & .76 & 4.39 & .67 & 4.28 & 1.07 & 4.38 & .71 & 4.49 & .78 \\
\hline \multirow[t]{2}{*}{ Total average for 47 items } & $\mathbf{A}$ & 3.85 & 1.08 & 3.98 & 1.09 & 3.46 & 1.16 & 3.70 & 1.24 & 3.52 & 1.17 & 3.70 & 1.15 \\
\hline & $\mathbf{E}$ & 4.32 & .83 & 4.15 & 1.03 & 4.09 & .89 & 4.09 & .98 & 3.88 & 1.00 & 4.11 & .95 \\
\hline
\end{tabular}

Note: $\mathrm{A}=$ Actual; $\mathrm{E}=$ Expected. 
Tables 8-15 reveal that the combined group means of all 47 expected (E) roles are higher than the combined group means of all 47 actual (A) roles.

Item 47-E, "Focus on Christian philosophy of institution," has the highest combined mean for all groups $(M=4.88)$ with the lowest combined standard deviation $(S D=0.32)$ among multiple statistics. This shows that the groups' very high expectations regarding this role were quite in agreement among them.

Item 24-E, "Help formulate and adhere to institution's educational philosophy," was rated second highest among all groups $(M=4.87)$. This shows that the status groups expected this particular role to be the second most important function of the presidents.

Three groups rated four items highest across the variables. See Table 16.

Table 16. Items with highest ratings by groups

\begin{tabular}{|c|c|c|c|c|c|c|c|}
\hline \multirow[t]{2}{*}{ Item } & \multicolumn{3}{|c|}{$\begin{array}{l}\text { Board } \\
\text { Chair }\end{array}$} & \multicolumn{2}{|c|}{ President } & \multicolumn{2}{|c|}{$\begin{array}{c}\text { Student } \\
\text { Service VP }\end{array}$} \\
\hline & & $M$ & $S D$ & $M$ & $S D$ & $M$ & $S D$ \\
\hline \multirow[t]{2}{*}{ 17. Prepare agenda for board of trustees' meetings } & $\mathbf{A}$ & & & 5.00 & .00 & & \\
\hline & $\mathbf{E}$ & & & 5.00 & .00 & & \\
\hline 24. Formulate \& adhere to educational philosophy & $\mathbf{E}$ & 5.00 & .00 & & & & \\
\hline 38. Represent institution to churches & $\mathbf{E}$ & & & & & 5.00 & .00 \\
\hline
\end{tabular}

Note: $\mathrm{A}=$ Actual; $\mathrm{E}=$ Expected.

The lowest actual group mean was $M=2.10$ for Item 7-A, "Know every student personally." The expected group mean for this item was $M=2.41$, which also was the lowest among the expected group means across the comparisons. This indicates that groups' expectations for presidents to "know every student personally" were low.

Table 17 shows that the board chairs rated 14 actual items and 24 expected items 
higher than all four groups, having the same means $M=4.56$ for Item $41-\mathrm{E}$ (Table 13) with the academic vice presidents, and $M=4.94$ for Item $47-\mathrm{E}$ with the presidents (Table 14).

Table 17. Item ratings by board chairs higher than all groups, by rank order of means

\begin{tabular}{|c|c|c|c|c|c|}
\hline \multicolumn{3}{|l|}{ Actual } & \multicolumn{3}{|l|}{ Expected } \\
\hline Item & $M$ & $S D$ & Item & $M$ & $S D$ \\
\hline 18. Preside over faculty meetings & 4.50 & .86 & 24. Adhere to ed. philosophy & 5.00 & .00 \\
\hline 31. Present physical needs to board & 4.44 & .70 & 46. Manifest Christ-like character & 4.94 & .24 \\
\hline 9. Recruit faculty & 4.39 & .70 & 43. Ensure students' spiritual life & 4.83 & .51 \\
\hline 13. Review accreditation reports & 4.06 & .87 & 21. Review organizational chart & 4.78 & .43 \\
\hline 32. Plan for new buildings & 4.06 & 1.00 & 9. Recruit faculty & 4.72 & .46 \\
\hline 35. Follow physical master plans & 4.06 & 1.63 & 26. Prepare annual budget & 4.72 & .46 \\
\hline 19. Structure admission standards & 3.67 & .77 & 44. Ensure faculty's spiritual life & 4.72 & .57 \\
\hline 2. Work with student leaders & 3.61 & .92 & 18. Preside over faculty meetings & 4.61 & 1.04 \\
\hline 12. Suggest academic changes & 3.56 & 1.15 & 3. Dialog with students & 4.56 & .51 \\
\hline 5. Administer student discipline & 3.50 & 1.29 & 13. Review accreditation reports & 4.50 & .71 \\
\hline 34. Do major equipment purchase & 3.50 & 1.42 & 45. Delegate religious programs & 4.39 & 1.09 \\
\hline 33. Plan for repairs $\&$ alterations & 3.44 & 1.25 & 11. Guard academic freedom & 4.33 & .77 \\
\hline 8. Serve as mentor of students & 3.33 & 1.08 & 35. Follow physical master plans & 4.28 & 1.07 \\
\hline \multirow[t]{11}{*}{ 10. Be faculty's academic leader } & 3.22 & 1.48 & 1. Recruit students & 4.11 & .90 \\
\hline & & & 15. Collaborate in-service training & 4.11 & .96 \\
\hline & & & 16. Carry out policies of faculty & 4.11 & 1.08 \\
\hline & & & 5. Administer student discipline & 4.11 & 1.28 \\
\hline & & & 2. Work with student leaders & 4.06 & .80 \\
\hline & & & 19. Structure admission standards & 4.06 & .87 \\
\hline & & & 12. Suggest academic changes & 3.78 & 1.22 \\
\hline & & & 10. Be faculty's academic leader & 3.67 & 1.41 \\
\hline & & & 4. Visit students in dorms & 3.44 & 1.29 \\
\hline & & & 6. Teach some classes & 3.00 & 1.68 \\
\hline & & & 7. Know students personally & 2.83 & 1.54 \\
\hline
\end{tabular}

Table 18 shows that the presidents rated 23 actual items and 2 expected items higher than all four groups, with Item 22 for actual role having the same mean as that of the finance vice presidents (Table 10). 
Table 18. Item ratings by presidents higher than all groups, by rank order of means

\begin{tabular}{rlrr}
\hline Item & & $M$ & $S D$ \\
\hline & & & \\
17-A & Prepare agenda for board of trustees' meetings & 5.00 & .00 \\
17-E & Prepare agenda for board of trustees' meetings & 5.00 & .00 \\
42-E & Listen to constituency and alumni & 4.94 & .24 \\
47-A & Focus on Christian philosophy of institution & 4.94 & .24 \\
38-A & Represent institution to churches of the constituency & 4.88 & .33 \\
46-A & Manifest Christ-like character in spiritual leadership roles & 4.82 & .53 \\
42-A & Listen to constituency and alumni & 4.76 & .44 \\
39-A & Present reports about institution to community and alumni & 4.76 & .66 \\
43-A & Assume primary responsibility for promotion of religious life among students & 4.71 & .69 \\
25-A & Delegate responsibility, authority, and needed resources to administrators & 4.71 & .77 \\
23-A & Comply with legal mandates and laws in addressing grievances & 4.59 & 1.00 \\
41-A & Associate closely with alumni groups & 4.35 & .86 \\
21-A & Take a leading role to prepare, revise, and follow organizational chart & 4.35 & 1.11 \\
30-A & Accept accountability to board for school's financial operation & 4.35 & 1.17 \\
44-A & Assume primary responsibility for promotion of religious life for faculty/staff & 4.29 & 1.26 \\
3-A & Dialogue frequently with students & 4.24 & .75 \\
36-A & Ascertain that campus grounds are well-kept through appropriate officer & 4.18 & 1.13 \\
11-A & Guard faculty's academic freedom & 4.06 & 1.20 \\
16-A & Carry out policies and regulations of faculty and staff & 4.06 & 1.30 \\
27-A & Ensure that institution's operating budget, after approval by board, is adhered to & 4.00 & 1.32 \\
29-A & Recommend to board the salary changes for faculty/staff positions & 3.88 & 1.58 \\
45-A & Take ultimate responsibility for planning chapels, and other religious programs & 3.71 & 1.40 \\
1-A & Participate in student recruitment & 3.65 & 1.17 \\
6-A & Teach some classes to maintain contact with students & 2.94 & 1.56 \\
4-A & Visit students periodically in dorms during their dorm worships & 2.88 & 1.32 \\
\hline & Actual E Expected & & \\
\hline
\end{tabular}

Note: $\mathrm{A}=$ Actual; $\mathrm{E}=$ Expected.

The finance vice presidents did not rate any actual role item or expected role item higher than any other respondents. Conversely, the finance vice presidents rated 19 actual role items and 21 expected role items lower than all four groups. See Tables 8-14. The student service vice presidents rated 9 actual role items and 7 expected role items higher than all the four groups. See Table 19. 
Table 19. Item ratings by student service VPs higher than all groups, by rank order of means

\begin{tabular}{|c|c|c|c|c|c|}
\hline \multicolumn{3}{|l|}{ Actual } & \multicolumn{3}{|l|}{ Expected } \\
\hline Item & $M$ & $S D$ & Item & $M$ & $S D$ \\
\hline 40. Represent school to public & 4.77 & .60 & 38. Represent school to churches & 5.00 & .00 \\
\hline 24. Adhere to ed. philosophy & 4.69 & .48 & 40. Represent school to public & 4.92 & .28 \\
\hline 20. Strategic planning for school & 4.54 & .97 & 39. Report to community & 4.85 & .38 \\
\hline 14. Hiring, promotion, dismissal & 4.46 & .97 & 23. Address grievances & 4.77 & .44 \\
\hline 37. Fund-raising for school & 4.31 & 1.11 & 22. Foster research climate & 4.54 & .66 \\
\hline 26. Prepare annual budget & 4.31 & 1.18 & 8. Serve as mentor of students & 4.00 & 1.00 \\
\hline 15. Collaborate in-service training & 3.85 & 1.21 & 33. Plan for repairs \& alterations & 3.54 & 1.39 \\
\hline 28. Present financial statement & 3.31 & 1.44 & & & \\
\hline 7. Know students personally & 2.46 & 1.45 & & & \\
\hline
\end{tabular}

Table 20 shows that the academic vice presidents rated 12 expected items higher than all the four groups. They did not rate any actual items higher than other respondents.

The result indicates that their expectations on these items were higher than others.

Table 20. Item ratings by academic VPs higher than all groups, by rank order of means

\begin{tabular}{lrr}
\hline Item & $M$ & $S D$ \\
\hline 25. Delegate responsibility, authority, and needed resources to appropriate administrators & 4.94 & .25 \\
37. Assist in fund-raising for support of institution & 4.94 & .25 \\
20. Take responsibility in strategic planning for institution & 4.88 & .34 \\
30. Accept accountability to board for school's financial operation & 4.81 & .54 \\
27. Ensure that institution's operating budget, after approval by board, is adhered to & 4.69 & .60 \\
14. Recommend to board for hiring, promotion, demotion \&dismissal of faculty \& staff & 4.69 & .79 \\
31. Present to board the physical needs of institution & 4.69 & 1.01 \\
32. Direct the preparation of plans for new buildings and installations as needed & 4.31 & .95 \\
36. Ascertain that campus grounds are well-kept through appropriate officer & 4.31 & .95 \\
29. Recommend to board the salary changes for facuity/staff positions & 4.06 & 1.18 \\
28. Present annual audited financial statements to board & 3.81 & 1.33 \\
34. Ensure that physical plant master plans are current and followed closely & 3.75 & 1.53 \\
\hline
\end{tabular}


Table 21 presents 9 items, perceived by the presidents, having lower expected means than actual means. This could be interpreted that the presidents did not expect to do any better than their present actual performances on these roles.

Table 21 . Item ratings by presidents having lower expected means than actual means

\begin{tabular}{|c|c|c|c|c|}
\hline \multirow{2}{*}{ Item } & \multicolumn{2}{|c|}{ Actual } & \multicolumn{2}{|c|}{ Expected } \\
\hline & $\bar{M}$ & $\overline{S D}$ & $\bar{M}$ & $\overline{S D}$ \\
\hline 1. Participate in student recruitment & 3.65 & 1.17 & 3.53 & 1.18 \\
\hline 2. Work closely with student government leaders & 3.59 & 1.23 & 3.53 & 1.28 \\
\hline 3. Dialogue frequently with students & 4.24 & .75 & 4.00 & 1.12 \\
\hline 5. Take ultimate responsibility for student discipline & 3.00 & 1.58 & 2.94 & 1.39 \\
\hline 6. Teach some classes to maintain contact with students & 2.94 & 1.56 & 2.88 & 1.58 \\
\hline 9. Participate in faculty recruitment & 4.24 & .90 & 4.18 & .81 \\
\hline 15. Collaborate for advanced in-service training for faculty and staff & 3.82 & 1.01 & 3.65 & 1.11 \\
\hline 18. Preside over general faculty meetings & 4.12 & 1.36 & 4.06 & 1.20 \\
\hline 36. Ascertain that campus grounds are well-kept & 4.18 & 1.13 & 4.12 & 1.22 \\
\hline
\end{tabular}

Table 22 shows that 2 items, as perceived by the student service vice presidents, have lower expected means than actual means. This could mean that they did not expect the presidents to do better than what they were perceived doing in these areas.

Table 22. Item ratings by student service VPs having lower expected means than actual means

\begin{tabular}{|c|c|c|c|c|}
\hline \multirow{2}{*}{ Item } & \multicolumn{2}{|c|}{ Actual } & \multicolumn{2}{|c|}{ Expected } \\
\hline & $\bar{M}$ & $S D$ & $\bar{M}$ & $\overline{S D}$ \\
\hline 1. Participate in student recruitment & 3.46 & 1.45 & 3.31 & 1.32 \\
\hline 6. Teach some classes to maintain contact with students & 2.00 & 1.29 & 1.46 & 1.61 \\
\hline
\end{tabular}


Item 9, "Participate in faculty recruitment," perceived by the finance vice presidents, and Item 18, "Preside over general faculty meetings," perceived by the academic vice presidents, have slightly lower expected means than their actual means for those items. These perceptions could mean that the said groups did not think that the presidents' expected role responsibilities should exceed the levels of presidents' actual role performances.

Seven items, perceived by the presidents, have the same means for actual and expected roles with the same standard deviations for each pair of items. See Table 23.

Table 23. Item ratings by presidents having same means and same standard deviations for actual and expected roles

\begin{tabular}{|c|c|c|c|c|}
\hline \multirow[b]{2}{*}{ Item } & \multicolumn{2}{|c|}{ Actual } & \multicolumn{2}{|c|}{ Expected } \\
\hline & $\bar{M}$ & $S D$ & $\bar{M}$ & $\overline{S D}$ \\
\hline 17. Prepare agenda for board of trustees' meetings & 5.00 & .00 & 5.00 & .00 \\
\hline 28. Present annual audited financial statements to board & 3.18 & 1.59 & 3.18 & 1.59 \\
\hline 31. Present to board the physical needs of institution & 4.29 & 1.10 & 4.29 & 1.10 \\
\hline 33. Plan for major repairs \& alterations of plant, buildings \& equipment & 2.65 & 1.46 & 2.65 & 1.46 \\
\hline 40. Represent institution as official spokesperson to public & 4.76 & .75 & 4.76 & .75 \\
\hline 46. Manifest Christ-like character in spiritual leadership roles & 4.82 & .53 & 4.82 & .53 \\
\hline 47. Focus on Christian philosophy of institution & 4.94 & .24 & 4.94 & .24 \\
\hline
\end{tabular}

These indicate that there were no differences in perceptions among the presidents regarding their actual role performances in 2004-2005 and their expected role responsibilities for these 7 items. No other groups exhibited this kind of identical perceptions. 
Item 17, "Prepare agenda for board of trustees' meetings," perceived by the finance vice presidents, has the same means $(M=4.57)$ for both actual and expected roles with the same standard deviations $(S D=0.65)$. This suggests that there are no differences in perceptions by the finance vice presidents between the actual role performances in 2004-2005 and expected role responsibilities of the presidents.

Table 24 shows similar means with dissimilar standard deviations for each of the five pairs of actual and expected role items. These items are: Item 5 under student service vice presidents and finance vice presidents; Item 6 under student service vice presidents, and Items 16, 19, and 43 under presidents. These statistics indicate that there were no differences in perceptions and expectations as far as the means were concerned, but the groups' response variances differed among themselves.

Table 24. Items with same means for actual and expected roles but different standard deviations

\begin{tabular}{|c|c|c|c|c|c|c|c|}
\hline & & \multicolumn{2}{|c|}{ President } & \multicolumn{2}{|c|}{$\begin{array}{l}\text { Student } \\
\text { Service VP }\end{array}$} & \multicolumn{2}{|c|}{$\begin{array}{l}\text { Finance } \\
\text { VP }\end{array}$} \\
\hline & & $M$ & $S D$ & $M$ & $S D$ & $M$ & $S D$ \\
\hline 5. Take responsibility for student discipline & $\begin{array}{l}\mathbf{A} \\
\mathbf{E}\end{array}$ & & & $\begin{array}{l}3.00 \\
3.00\end{array}$ & $\begin{array}{l}1.68 \\
1.73\end{array}$ & $\begin{array}{l}2.86 \\
2.86\end{array}$ & $\begin{array}{l}1.41 \\
1.56\end{array}$ \\
\hline 6. Teach classes for contact with students & $\begin{array}{l}\mathbf{A} \\
\mathbf{E}\end{array}$ & & & & & $\begin{array}{l}2.93 \\
2.93\end{array}$ & $\begin{array}{l}1.69 \\
1.54\end{array}$ \\
\hline 16. Carry out policies \& regulations of faculty & $\begin{array}{l}\mathbf{A} \\
\mathbf{E}\end{array}$ & $\begin{array}{l}4.06 \\
4.06\end{array}$ & $\begin{array}{l}1.30 \\
1.34\end{array}$ & & & & \\
\hline $\begin{array}{l}\text { 19. Take responsibility in structuring } \\
\text { student admission standards }\end{array}$ & $\begin{array}{l}\mathbf{A} \\
\mathbf{E}\end{array}$ & $\begin{array}{l}2.71 \\
2.71\end{array}$ & $\begin{array}{l}1.45 \\
1.31\end{array}$ & & & & \\
\hline $\begin{array}{l}\text { 43. Assume primary responsibility for } \\
\text { promotion of religious life of students } \\
\text { at the institution via delegation }\end{array}$ & $\begin{array}{l}\mathbf{A} \\
\mathbf{E}\end{array}$ & $\begin{array}{l}4.71 \\
4.71\end{array}$ & $\begin{array}{l}.69 \\
.59\end{array}$ & & & & \\
\hline
\end{tabular}

Note: A=Actual; E=Expected. 
Item 6-A, "Teach some classes to maintain contact with students," shows highest standard deviation $(M=1.69)$ among all variables, as viewed by the board chairs and finance vice presidents. This is indicative of their wide variances in perceptions regarding this particular role item. Item 6-A, "Teach some classes to maintain contact with students," and Item 34-E, "Recommend to board for equipment acquisition," have the highest overall standard deviation $(S D=1.55)$ across the variables.

Physical facilities-related functions show higher standard deviations among the groups in all items for both actual and expected roles. Student-related roles also have high standard deviations, but Item 3, "Dialogue frequently with students," shows high expectation $(M=4.19)$ with standard deviation of $S D=0.87$.

All six items of community- and alumni-related functions present high actual means and high expected means for each status group with low standard deviations. This shows that there are not many variances in perceptions among the groups between the presidents' actual role performances and their expected role responsibilities.

Most of the items of the student-related functions and physical facilities-related functions have high standard deviations for both actual and expected roles, indicating greater variances in perceptions and expectations regarding these role items. The greatest difference between standard deviations of actual and expected roles is found in Item 25 , "Delegate responsibility, authority, and needed resources to appropriate administrators and support their roles." This shows that the groups varied greatly in their perceptions regarding the actual role performance $(S D=1.14)$, but they did not differ much in their expectations $(S D=0.53)$ of that particular item. Items $4,5,7,8,12,18,19,28$, and 34 
have higher standard deviations for expected roles than actual roles, but the expected means are higher than the actual means for these items.

In short, Tables 8-14 reveal that out of a total of 235 expected (E) means across the five groups (board chairs, presidents, academic vice presidents, student service vice presidents, and finance vice presidents), 208 expected role items were found to be higher than actual (A) role means. Fourteen expected means were tied with their actual means, while 13 expected means were lower than their actual means in the pairs.

\section{Research Questions 1 and 2}

Results derived from the treatment of the data are presented in appropriate tables and discussed in the related narrative in this chapter in order of purposes, their research questions, and their corresponding null hypotheses.

Purpose 1: Purpose 1 of this study was to determine the actual role performances in 2004-2005 of the presidents of the Seventh-day Adventist senior colleges and universities around the world offering graduate programs on their campuses. Research Question 1 corresponded to this purpose.

\section{Research Question 1}

Research Question 1 stated: What are the actual role performances of presidents of Seventh-day Adventist senior colleges and universities around the world, as perceived by their board chairs, presidents, academic vice presidents, student service vice presidents, and finance vice presidents?

To address this purpose and the corresponding research question, descriptive 
statistics obtained from SPSS output were utilized to examine the means and the standard deviations of dependent variables for 47 actual role items of presidents as viewed by the status groups. Seventy-eight administrators responded to the questionnaire on a Likerttype scale of 1 through 5 , with a response of 5 indicating their perception of presidents' very strong performance of actual roles, and a response of 1 indicating their perception of presidents' very weak performance of actual roles. The intermediate numbers 2,3 , and 4 indicate weak performance, moderate performance, and strong performance, respectively, of presidents' actual roles. These activities are listed in Table 25 in four levels of actual role performances in order of the highest mean to the lowest mean.

If the overall mean scores ranged from $M=4.51-M=5.00$, the groups' perception was that the presidents' actual role performances on the specified functions were very strong or excellent. This is Level 1: Very Strong Role Performance.

If the mean scores were from $M=3.51-M=4.50$, the perception was that the presidents' actual performances on the specified roles were strong. "Strong" means the presidents were doing their job well. This is Level 2: Strong Role Performance.

A range of $M=2.51$ to $M=3.50$ equates to a perception of moderate role performance, meaning neither strong nor weak. "Neither strong nor weak" means when respondents did not agree or disagree regarding presidents' role performances of the specified items. This is Level 3: Moderate Role Performance.

If the means were between $M=1.51$ and $M=2.50$, the groups' perception was that the presidents' performances were weak. "Weak" represents respondents' disagreement regarding presidents' actual role performance. This is Level 4: Weak Role Performance. 
Table 25. Groups' perceptions of presidents' actual role performance by rank order of means $(N=78)$

\begin{tabular}{lllll}
\hline Item & Rank & Role & $M$ & $S D$ \\
\hline
\end{tabular}

\section{Level 1: Very Strong Role Performance \\ $(M=4.51-M=5.00)$}

$47 \quad 1.5 \quad$ Focus on Christian philosophy of institution

\section{Level 2: Strong Role Performance \\ $(M=3.51-M=4.50)$}

Represent institution as its official spokesperson to public

Manifest Christ-like character in spiritual leadership roles .

Help formulate and adhere to educational philosophy

Represent institution to churches of the constituency

Have responsibility in strategic planning for institution

Present reports about institution to community and alumni

Comply with legal mandates and laws in addressing grievances

Listen to constituency and alumni ...

Recommend to board for hiring, promotion, demotion, and dismissal of faculty and staff.

Delegate responsibility, authority, and needed resources to appropriate administrators and support their roles

Assume primary responsibility for promotion of students' religious life at the institution via delegation

Present to board the physical needs of institution

Accept accountability to board for financial operation of institution ........... 4.05

Take a leading role to prepare, revise, and follow organizational chart ....... 4.04

Preside over general faculty meetings ..................................................... 3.99

Participate in the preparation of institution's annual operating budget ........ 3.92

Foster research and intellectual climate

Assume primary responsibility for promotion of religious life of faculty and staff at the institution via delegation

Dialogue frequently with students

Work with faculty in reviewing accrediting agency's reports ...................... 3.73

Ascertain that campus grounds are well kept through appropriate officer ... 3.73

Ensure that institution's operating budget, after approval by board,

is adhered to

Guard faculty's academic freedom ... 
Table 5--Continued.

\begin{tabular}{|c|c|c|c|}
\hline Item & Rank & Role & $S D$ \\
\hline \multicolumn{4}{|c|}{$\begin{array}{l}\text { Level 3: Moderate Role Performance } \\
\qquad(M=2.51-M=3.50)\end{array}$} \\
\hline 32 & 31 & $\begin{array}{l}\text { Direct the preparation of plans for new buildings and installations } \\
\text { as needed }\end{array}$ & 1.44 \\
\hline 15 & 32 & Collaborate for advanced in-service training for faculty and staff ...................42 & 1.25 \\
\hline 45 & 33 & $\begin{array}{l}\text { Take ultimate responsibility for planning midweek chapels, vespers, } \\
\text { weeks of spiritual emphasis and other religious programs on } \\
\text { campus via delegation for spiritual nurture }\end{array}$ & 1.34 \\
\hline 1 & 34 & 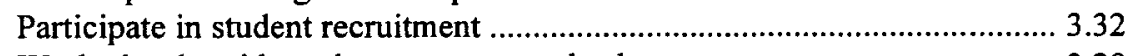 & 1.26 \\
\hline 2 & 35 & 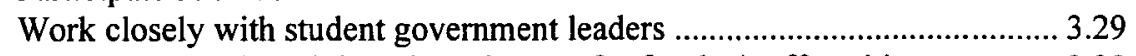 & 1.23 \\
\hline 29 & 36 & Recommend to board the salary changes for faculty/staff positions ...............3.28 & 1.42 \\
\hline 28 & 37 & Present annual audited financial statements to board ..................................... 3.12 & 1.47 \\
\hline 33 & 38.5 & Plan for major repairs or alterations of plant, buildings and equipment ....... 3.05 & 1.39 \\
\hline 34 & 38.5 & Recommend to board for equipment acquisition ........................................... 3.05 & 1.50 \\
\hline 19 & 40.5 & Have responsibility for structuring student admission standards ................... 2.97 & 1.21 \\
\hline 10 & 40.5 & 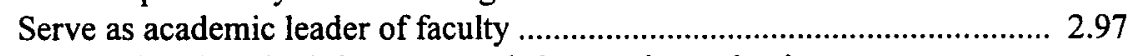 & 1.26 \\
\hline 12 & 42 & $\begin{array}{l}\text { Responsible in submitting proposed changes in academic matters } \\
\text { to faculty for approval }\end{array}$ & 1.31 \\
\hline 5 & 43 & 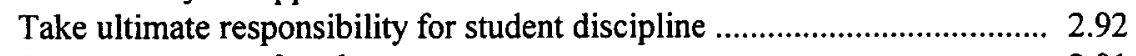 & 1.45 \\
\hline 8 & 44 & Serve as mentor of students & 1.27 \\
\hline 4 & 45 & $\begin{array}{l}\text { Visit students periodically in dorms during dorm worships .......................... } 2.55 \\
\qquad \begin{array}{c}\text { Level 4: Weak Role Performance } \\
(M=1.51-M=2.50)\end{array}\end{array}$ & 1.24 \\
\hline 6 & 46 & Teach some classes to maintain contact with students ..................................... 2.45 & 1.55 \\
\hline 7 & 47 & 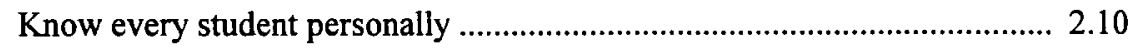 & 1.19 \\
\hline
\end{tabular}

The overall group mean scores ranging from $M=1.00-M=1.50$ indicate that the groups' perceptions regarding the presidents' actual role performances on the specified functions are very weak. "Very Weak" indicates respondents' strong disagreement regarding presidents' actual role performances. Descriptive statistics showed that there were no "very weak performance" items. Hence, Level 5 is not included in Table 25 . 
Very strong role performance

Out of a total of 47 items listed in the questionnaire, 2 items (4.3\%)

were found to have taken the topmost rank, falling into the category of very strong performance ( $M=4.51-M=5.00)$, meaning that perceptions regarding these items were in very strong agreement among the groups. These two items are:

47. Focus on Christian philosophy of education $(M=4.64, S D=0.77)$, and

17. Prepare agenda for board of trustees' meetings $(M=4.64, S D=0.79)$.

These 2 items belonged to the sections under spiritual life-related functions and general administrative functions, respectively, having an equal rank of 1.5.

Strong role performance

Twenty-eight items (59.5\%) out of 47 items were perceived to be strong in terms of presidents' actual role performances in 2004-2005. Item 40, "Represent institution as its official spokesperson to public" $(M=4.49, S D=0.88)$, and Item 16 , "Carry out policies and regulations of faculty and staff' $(M=3.62, S D=1.34)$, were placed on top and in the bottom, taking rank 3 and rank 30 , respectively, in the strong performance section, based upon their mean scores and standard deviations.

Student-Related Functions: Only item "Dialogue frequently with students" $(M=3.73, S D=1.14)$ was rated to have strong performance $(12.5 \%)$.

Employee-Related Functions: Five out of eight items (62.5\%) were positioned in the strong performance category. These items, by rank order of means, are:

14. Recommend to board for hiring, promotion, demotion, and dismissal of 
faculty and $\operatorname{staff}(M=4.14, S D=1.14)$

9. Participate in faculty recruitment $(M=4.05, S D=0.99)$

13. Work with faculty in reviewing accrediting agency's report $(M=3.73$, $S D=1.15)$

11. Guard faculty's academic freedom $(M=3.72, S D=1.04)$, and

16. Carry out policies and regulations of faculty and staff $(M=3.62, S D=1.34)$.

General Administrative Functions: Seven out of nine items (77.8\%) were rated by status groups as strong performance in 2004-2005. These items, by rank order of means, are:

24. Help formulate and adhere to institution's educational philosophy $(M=4.44$, $S D=0.92)$

20. Take responsibility in strategic planning for institution $(M=4.29, S D=1.05)$

23. Comply with legal mandates and laws in addressing grievances $(M=4.26$, $S D=0.93)$

25. Delegate responsibility, authority, and needed resources to appropriate administrators and support their roles $(M=4.14, S D=1.14)$

21. Take a leading role to prepare, revise, and follow organizational chart $(M=4.04, S D=1.11)$

18. Preside over general faculty meetings $(M=3.99, S D=1.32)$, and

22. Foster research and intellectual climate for institution $(M=3.81, S D=1.13)$.

Finance-Related Functions: Of the five items, three items were viewed to have been receiving strong leadership $(60.0 \%)$. These items, by rank order of means, are: 
30. Accept accountability to board for school's financial operation $(M=4.05$, $S D=1.14)$

26. Participate in the preparation of annual budget of institution $(M=3.92$, $S D=1.29)$, and

27. Ensure that institution's budget, after approval by board, is adhered to $(M=3.73, S D=1.20)$.

Physical Facilities-Related Functions: Three out of six items (50.0\%) were perceived to be strong in terms of presidents' actual role performances in 2004-2005:

31. Present to board the physical needs of institution $(M=4.06, S D=1.24)$

36. Ascertain that campus grounds are well-kept through appropriate officer and administrator $(M=3.73, S D=1.18)$, and

35. Ensure that physical plant master plans are current and followed as closely as possible $(M=3.63, S D=1.36)$.

Community- and Alumni-Related Functions: All six items (100\%) were rated as strong performance. These items, by rank order of means, are:

40. Represent institution as its official spokesperson to public $(M=4.49$, $S D=0.88)$

38. Represent institution to churches of the constituency $(M=4.40, S D=0.92)$

39. Present reports about institution to community and alumni $(M=4.28$, $S D=0.07)$

42. Listen to constituency and alumni $(\mathrm{M}=4.23, \mathrm{SD}=1.04)$

37. Assist in raising funds for support of institution $(M=4.09, S D=1.15)$, and 
41. Associate closely with alumni groups $(M=4.05, S D=1.01)$.

Spiritual Life-Related Functions: Three out of five items (60\%) were found to have fallen into strong performance category. These items, by rank order of means, are:

46. Manifest Christ-like character in spiritual leadership roles $(M=4.46$, $S D=0.80$ )

43. Assume primary responsibility for promotion of students' religious life at the institution via delegation $(M=4.08, S D=1.10)$, and

44. Assume primary responsibility for promotion of religious life of faculty and staff at the institution via delegation $(M=3.77, S D=1.19)$.

Moderate role performance

A total of 15 items (31.9\%) came under moderate performance category based on the means $(M=2.51-M=3.50)$ calculated from the responses of the five groups surveyed to determine the extent of actual role performances of presidents in 2004-2005. Item 32, "Direct the preparation of plans for new buildings and installations as needed," $(M=3.50)$, and Item 4, "Visit students in dorms periodically during dorm worships," $(M=2.55)$ ranked on top and in the bottom, taking rank 31 and rank 45 , respectively, in the moderate performance section, based upon their mean scores.

Student-Related Functions: Five out of eight items (62.5\%) were categorized as moderate performance. These items, by rank order of means, are:

1. Participate in student recruitment $(M=3.32, S D=1.26)$

2. Work closely with student government leaders $(M=3.29, S D=1.23)$ 
5. Take ultimate responsibility for student discipline $(M=2.92, S D=1.45)$

8. Serve as mentor of students $(M=2.91, S D=1.27)$, and

4. Visit students periodically in dorms during dorm worships $(M=2.55$, $S D=1.24)$

Employee-Related Functions: Three out of eight items (37.5\%) fell under moderate performance category. These items, by rank order of means, are:

15. Collaborate for advanced in-service training for faculty and staff $(M=3.42$, $S D=1.25)$

10. Serve as academic leader of faculty $(M=2.97, S D=1.26)$, and

12. Have responsibility in submitting proposed changes in academic matters to faculty for approval $(M=2.96, S D=1.31)$.

General Administrative Functions: Only one out of nine items (11.1\%) fell under moderate performance category. That item was:

19. Take responsibility in structuring student admission standards $(M=2.97$, $S D=1.21)$

Finance-Related Functions: Two out of five items $(40.0 \%)$ were rated as moderate performance. These items, by rank order of means, are:

29. Recommend to board the salary changes for positions $(M=3.28, S D=1.42)$

28. Present annual audited financial statements to board $(M=3.12, S D=1.47)$. Physical Facilities-Related Functions: Three out of six items (50.0\%) were perceived by the groups to be of moderate performance as far as presidents' actual roles in 2004-2005 were concerned. These items, by rank order of means, are: 
32. Direct the preparation of plans for new buildings and installations as needed $(M=3.50, S D=1.44)$

33. Plan for major repairs and alterations of plant, buildings, and equipment $(M=3.05, S D=1.39)$, and

34. Recommend to board for equipment acquisition $(M=3.05, S D=1.50)$. Spiritual Life-Related Functions: One out of five items (20.0\%) fell in the moderate performance section. That item was:

45. Take ultimate responsibility for planning midweek chapels, vespers, weeks of spiritual emphasis and other religious programs of the institution via delegation for spiritual nurture $(M=3.35, S D=1.34)$.

Weak role performance

Two items came under the weak performance category $(M=1.51-M=2.50)$ :

6. Teach some classes to maintain contact with students $(M=2.45, S D=1.55)$. Presidents' combined mean was the highest $(M=2.94)$, while academic vice presidents' combined mean was the lowest $(M=1.75)$ for this role item. See Table 8 .

7. Know every student personally $(M=2.10, S D=1.19)$. Student service vice presidents' combined mean was the highest $(M=2.46)$, while academic vice presidents' combined mean was the lowest $(M=1.75)$ for this role item (Table 8$)$.

Both of these items, 6 and 7, belonged to student-related functions. These two mean scores indicate the five groups' strong disagreement regarding presidents' actual role performance of these two items in 2004-2005. 
Table 26 provides a summary distribution data for the seven broad areas of presidents' functions in their five levels of actual performances, such as, very strong role performance, strong role performance, moderate role performance, weak role performance, and very weak role performance. The figures in the table represent the number of items falling into the five levels of actual role performances, based upon the combined mean scores computed from the status groups' responses.

Table 26. Summary distribution of number of items by levels of actual role performances and categories of functions, based on Table 25

\begin{tabular}{|c|c|c|c|c|c|c|}
\hline $\begin{array}{l}\text { Categories } \\
\text { of } \\
\text { Functions }\end{array}$ & $\begin{array}{c}\text { Level 1 } \\
\text { Very Strong } \\
\text { Role } \\
\text { Performance } \\
\text { Items }\end{array}$ & $\begin{array}{l}\text { Level 2 } \\
\text { Strong } \\
\text { Role } \\
\text { Performance } \\
\text { Items }\end{array}$ & $\begin{array}{c}\text { Level } 3 \\
\text { Moderate } \\
\text { Role } \\
\text { Performance } \\
\text { Items }\end{array}$ & $\begin{array}{l}\text { Level } 4 \\
\text { Weak } \\
\text { Role } \\
\text { Performance } \\
\text { Items }\end{array}$ & $\begin{array}{l}\text { Level } 5 \\
\text { Very Weak } \\
\text { Role } \\
\text { Performance } \\
\text { Items }\end{array}$ & $\begin{array}{l}\text { Total } \\
\text { Items }\end{array}$ \\
\hline $\begin{array}{l}\text { I. Student-Related } \\
\text { Functions }\end{array}$ & 0 & 1 & 5 & 2 & 0 & 8 \\
\hline $\begin{array}{l}\text { II. Employee-Related } \\
\text { Functions }\end{array}$ & 0 & 5 & 3 & 0 & 0 & 8 \\
\hline $\begin{array}{l}\text { III. General } \\
\text { Administrative } \\
\text { Functions }\end{array}$ & 1 & 7 & 1 & 0 & 0 & 9 \\
\hline $\begin{array}{l}\text { IV. Finance-Related } \\
\text { Functions }\end{array}$ & 0 & 3 & 2 & 0 & 0 & 5 \\
\hline $\begin{array}{l}\text { V. Physical Facilities- } \\
\text { Related Functions }\end{array}$ & 0 & 3 & 3 & 0 & 0 & 6 \\
\hline $\begin{array}{l}\text { VI. Community-and } \\
\text { Alumni-Related } \\
\text { Functions }\end{array}$ & 0 & 6 & 0 & 0 & 0 & 6 \\
\hline $\begin{array}{l}\text { VII. Spiritual Life- } \\
\text { Related Functions }\end{array}$ & 1 & 3 & 1 & 0 & 0 & 5 \\
\hline Totals & 2 & 28 & 15 & 2 & 0 & 47 \\
\hline Percentages & 4.3 & 59.5 & 31.9 & 4.3 & 0.0 & 100.0 \\
\hline
\end{tabular}


Table 26 shows that of the 47 items grouped in seven broad areas of functions the status groups perceived that $4.3 \%$ of the actual roles were performed with a very strong leadership during the 2004-2005 school year, $59.5 \%$ of the items fell in the category of strong performance, $31.9 \%$ of the activities were viewed to be of moderate performance, and only $4.3 \%$ of the functions were considered as weak performance. No item was perceived as a very weak actual role performance. None of the student-related, employee-related, finance-related, physical facilities-related, and community- and alumnirelated functions were carried out very strongly.

Purpose 2: Purpose 2 investigated the expected roles of presidents of Seventhday Adventist senior colleges and universities around the world that offer graduate programs on their campuses. Research Question 2 corresponded to this purpose.

\section{Research Question 2}

Research Question 2 stated: What are the expected role responsibilities of presidents of Seventh-day Adventist senior colleges and universities around the world as viewed by their board chairs, presidents, academic vice presidents, student service vice presidents, and finance vice presidents?

To address this purpose and the corresponding research question, descriptive statistics obtained from the SPSS output were utilized to examine the means and the standard deviations of the dependent variables for the 47 expected role responsibilities of presidents as viewed by the status groups. These functions were presented as reported by the 78 administrative team members in response to the questionnaires on a Likert-type 
scale of 1 through 5 , with a response of 5 indicating a very high role expectation of presidents' expected role responsibilities and a response of 1 indicating a very low expectation of presidents' function in specified expected role responsibilities. The intermediate numbers, 2,3 , and 4 indicate low, moderate, and high expectations, respectively, of presidents' expected role responsibilities. These activities are listed in Table 27 in four levels of expectations in order of the highest mean to the lowest mean.

The means ranging from $M=4.51-M=5.00$ indicated groups' very high role expectations of presidents' expected role responsibilities in the specified items. Very high role expectation represented status groups' very strong agreement that presidents should perform the specified role responsibilities with excellence. This is Level 1: Very High Role Expectation.

The means from $M=3.51$ to $M=4.50$ indicated the groups' high expectations for the roles. High expectation represented agreement by status groups regarding specified expected roles that presidents should carry out. This is Level 2: High Role Expectation. A range of $M=2.51-M=3.50$ equates to a perception of moderate expectation, meaning neither high nor low expectation. Moderate role expectation indicated that respondents neither agreed nor disagreed that presidents should or should not perform the specified expected role responsibilities. This is Level 3: Moderate Role Expectation.

If the mean scores fell between $M=1.51$ and $M=2.50$, the groups' perceptions regarding presidents' expected roles were low. Low role expectation represented a position of status groups that they disagree that presidents should perform the specified expected role items. This is Level 4: Low Role Expectation. 
Table 27. Groups' perceptions of presidents' expected role responsibilities by rank order of means $(N=78)$

\begin{tabular}{lllll}
\hline Item & Rank & Role & $M$ & $S D$ \\
\hline
\end{tabular}

\section{Level 1: Very High Role Expectation}

$$
(M=4.51-M=5.00)
$$

$\begin{array}{cc}47 & 1 \\ 24 & 2 \\ 38 & 3 \\ 46 & 4 \\ 17 & 5 \\ 37 & 6 \\ 20 & 7.5 \\ 40 & 7.5 \\ 42 & 9 \\ 25 & 10 \\ & \\ 39 & 11 \\ 30 & 12 \\ 23 & 13 \\ 21 & 14 \\ 41 & 15\end{array}$

Focus on Christian philosophy of institution ............................................. 4.88

Help formulate and adhere to institution's educational philosophy .............. 4.87

Represent institution to churches of the constituency .................................. 4.85

Manifest Christ-like character in spiritual leadership roles ......................... 4.83

Prepare agenda for board of trustees' meetings ......

Assist in raising funds for support of institution

4.77

.44

.60

Have responsibility in strategic planning for institution

4.76

.61

Represent institution as its official spokesperson to public .

4.76

.71

Listen to constituency and alumni

4.74

Delegate responsibility, authority, and needed resources to appropriate administrators and support their roles

Present reports about institution to community and alumni ...

Accept accountability to board for financial operation of institution ........... 4.62

Comply with legal mandates and laws in addressing grievances ................. 4.60

Take a leading role to prepare, revise, and follow organizational chart ....... 4.53

Associate closely with alumni groups

.75

\section{Level 2: High Role Expectation$$
(M=3.51-M=4.50)
$$

$\begin{array}{ll}43 & 16 \\ 27 & 17 \\ & \\ 14 & 18 \\ & \\ 26 & 19 \\ 44 & 20 \\ & \\ 31 & 21 \\ 22 & 22 \\ 13 & 23 \\ 9 & 24 \\ 3 & 25 \\ 11 & 26 \\ 35 & 27 \\ 36 & 28 \\ 18 & 29 \\ 45 & 30\end{array}$

Assume primary responsibility for promotion of students'

religious life at the institution via delegation...

Ensure that institution's operating budget, after approval by board, is adhered to

Recommend to board for hiring, promotion, demotion and dismissal of faculty and staff

Participate in the preparation of institution's annual operating budget ..

Assume primary responsibility for promotion of religious life of faculty and staff at the institution via delegation

Present to board the physical needs of the institution .....

Foster research and intellectual climate....

Work with faculty in reviewing accrediting agency's reports .................... 4.23

Participate in faculty recruitment ................................................................ 4.22

Dialogue frequently with students

Guard faculty's academic freedom

Ensure that physical plant master plans are current and followed closely .... 4.09

Ascertain that campus grounds are well kept through appropriate officer ....4.04

Preside over general faculty meetings ....

1.01

.95

.87

.87

1.08

1.12

Take ultimate responsibility for planning midweek chapels, vespers, weeks of spiritual emphasis and other religious programs on campus via delegation for spiritual nurture 
Table 27-Continued.

\begin{tabular}{|c|c|c|c|c|}
\hline Item & Rank & Role & $M$ & $S D$ \\
\hline & & $\begin{array}{l}\text { Level 2: High Role Expectation (contd.) } \\
\qquad(M=3.51-M=4.50)\end{array}$ & & \\
\hline 32 & 32 & $\begin{array}{l}\text { Direct the preparation of plans for new buildings and installations } \\
\text { as needed }\end{array}$ & 3.79 & 1.22 \\
\hline 29 & 33 & Recommend to board the salary changes for faculty/staff positions . & .3 .69 & 1.40 \\
\hline 2 & 34.5 & Work closely with student government leaders & .3 .65 & 1.14 \\
\hline 15 & 34.5 & Collaborate for advanced in-service training for faculty and staff ..... & .3 .65 & 1.23 \\
\hline 1 & 36 & Participate in student recruitment & .3 .59 & 1.12 \\
\hline
\end{tabular}

\section{Level 3: Moderate Role Expectation}

$$
(M=2.51-M=3.50)
$$

\begin{tabular}{|c|c|c|}
\hline 28 & 37 & Present annual audited financial statements to board ....................................38 \\
\hline 34 & 38 & 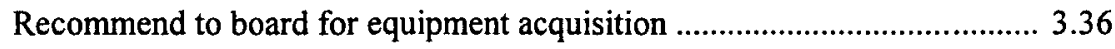 \\
\hline 8 & 39 & Serve as mentor of students \\
\hline 10 & 40 & Serve as academic leader of faculty \\
\hline 19 & 41 & Have responsibility for structuring student admission standards .................. 3.27 \\
\hline 12 & 42.5 & $\begin{array}{l}\text { Have responsibility in submitting proposed changes in academic matters } \\
\text { to faculty for approval }\end{array}$ \\
\hline & 42.5 & 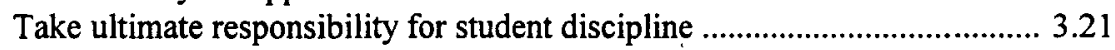 \\
\hline & 44 & buildings and equipment .... 3.17 \\
\hline & 45 & Visit students periodically in dorms during dorm worships .. \\
\hline & 46 & Teach some classes to maintain contact with students ............... \\
\hline
\end{tabular}

Level 4: Low Role Expectation

$(M=1.51-M=2.50)$

$\begin{array}{lll}7 & 47 & \text { Know every student personally }\end{array}$

2.41

1.32

The overall group mean scores ranging from $M=1.00$ to $M=1.50$ indicated a very low expectation of presidents' function in the specified role responsibilities. Very low expectation represented status groups' strong disagreement on the specified expected role items. Descriptive statistics showed that there were no very low expectation items. Hence, Level 5 is not included in Table 27. 
Very high role expectation

In Table 27, out of a total of 47 items, the respondents considered 15 (31.9\%) expected role items with very high expectations. Item 47, "Focus on Christian philosophy of institution," had the highest mean score of $M=4.88$ among the total group mean scores with the rank of 1 . This item showed the lowest standard deviation $(S D=0.32)$, indicating the least variances in the groups' expectations for this role item.

General Administrative Functions: From the 15 very high expectation items, six out of nine $(66.7 \%)$ expected roles belonged to general administrative functions category. These general administrative items, by rank order of means, are:

24. Help formulate and adhere to institution's educational philosophy $(M=4.87$, $S D=0.37)$

17. Prepare agenda for board of trustees' meetings $(M=4.81, S D=0.60)$

20. Take responsibility in institution's strategic planning $(M=4.76, S D=0.61)$

25. Delegate responsibility, authority, and needed resources to appropriate administrators and support their roles $(M=4.73, S D=0.53)$

23. Comply with legal mandates and laws in addressing grievances $(M=4.60$, $S D=0.74)$, and

21. Take a leading role to prepare, revise, and follow organizational chart $(M=4.53, S D=0.75)$.

Finance-Related Functions: Only one (20.0\%) out of five items of financerelated functions came under very high expectation. That item was:

30. Accept accountability to board for school's financial operation $(M=4.62$, 
$S D=0.84)$

Community- and Alumni-Related Functions: All six items (100\%) were rated as very high expectation items. These items, by rank order of means, are:

38. Represent institution to churches of the constituency $(M=4.85, S D=0.40)$

37. Assist in raising funds for support of institution $(M=4.77, S D=0.64)$

40. Represent institution as its official spokesperson to public $(M=4.76$, $S D=0.71)$

42. Listen to constituency and alumni $(M=4.74, S D=0.47)$

39. Present reports about institution to constituency and alumni $(M=4.67$, $S D=0.64)$, and

41. Associate closely with alumni groups $(M=4.51, S D=0.62)$.

Spiritual Life-Related Functions: Two out of five roles (40.0\%) were rated as very high expectation items. These items, by rank order of means, are:

47. Focus on Christian philosophy of institution $(M=4.88, S D=0.32)$, and

46. Manifest Christ-like character in spiritual leadership $(M=4.83, S D=0.44)$.

The high mean scores and low standard deviations in the very high expectation section revealed that the groups were in strong agreement regarding these 15 expected roles without much variances among them in their opinions.

High role expectation

Twenty-one items (44.7\%) fell under the high expectation section with mean scores ranging from $M=3.59$ to $M=4.47$. The lowest standard deviation was $S D=0.83$, 
while the highest standard deviation was $S D=1.40$ in this section. This indicates that there were many levels of variances among the groups in their perceptions. The groups, however, agreed on levels of expectations regarding these 21 role items. These 21 expected roles belonged to student-related functions ( 3 items, with ranks $25,34.5$, and 36); employee-related functions (6 items, with ranks 18, 23, 24, 26, 31, and 34.5); general administrative functions ( 2 items, with ranks 22 and 29); finance-related functions ( 3 items, with ranks 17, 19, and 33); physical facilities functions (4 items, with ranks 21, 27, 28 , and 32); and spiritual life-related functions ( 3 items, with ranks 16,20 , and 30 ).

Moderate role expectation

Ten items $(21.3 \%)$ were labeled as moderate expectation category because the groups neither agreed nor disagreed whether presidents should have these role responsibilities or not. They expected presidents to perform these roles moderately. The mean scores of these 10 items ranged from $M=2.73$ to $M=3.38$ with standard deviations rising up to $S D=1.55$. The high standard deviations in this section indicated that the groups differed much in their expectations regarding these items. The 10 expected roles belonged to student-related functions (4 items, with ranks $39,42.5,45$, and 46 ); employee-related functions ( 2 items, with ranks 40 and 42.5 ); general administrative functions ( 1 item, with rank 41); finance-related functions ( 1 item, with rank 37 ); and physical facilities-related functions ( 2 items, with ranks 38 and 44 ).

Low role expectation

Only one item, 7, "Know every student personally" $(M=2.41, S D=1.32)$ 
belonging to student-related functions, came under low expectation category. No item came under the very low expectation section.

Table 28 presents a summary distribution of data on expected roles in seven broad areas of function by different levels of expectation, based upon the means calculated from the subjects' responses to 47 questions.

Table 28. Summary distribution of number of items by levels of expected role responsibilities and categories of functions, based on Table 27

\begin{tabular}{|c|c|c|c|c|c|c|}
\hline $\begin{array}{l}\text { Categories } \\
\text { of } \\
\text { Functions }\end{array}$ & $\begin{array}{l}\text { Level } 1 \\
\text { Very High } \\
\text { Role } \\
\text { Expectation } \\
\text { Items }\end{array}$ & $\begin{array}{c}\text { Level 2 } \\
\text { High } \\
\text { Role } \\
\text { Expectation } \\
\text { Items }\end{array}$ & $\begin{array}{l}\text { Level } 3 \\
\text { Moderate } \\
\text { Role } \\
\text { Expectation } \\
\text { Items }\end{array}$ & $\begin{array}{c}\text { Level } 4 \\
\text { Low } \\
\text { Role } \\
\text { Expectation } \\
\text { Items }\end{array}$ & $\begin{array}{l}\text { Level } 5 \\
\text { Very Low } \\
\text { Role } \\
\text { Expectation } \\
\text { Items }\end{array}$ & $\begin{array}{l}\text { Total } \\
\text { Items }\end{array}$ \\
\hline $\begin{array}{l}\text { 1. Student-Related } \\
\text { Functions }\end{array}$ & 0 & 3 & 4 & 1 & 0 & 8 \\
\hline $\begin{array}{l}\text { II. Employee-Related } \\
\text { Functions }\end{array}$ & 0 & 6 & 2 & 0 & 0 & 8 \\
\hline $\begin{array}{l}\text { III. General } \\
\text { Administrative } \\
\text { Functions } \\
\end{array}$ & 6 & 2 & 1 & 0 & 0 & 9 \\
\hline $\begin{array}{l}\text { IV. Finance-Related } \\
\text { Functions }\end{array}$ & 1 & 3 & 1 & 0 & 0 & 5 \\
\hline $\begin{array}{l}\text { V. Physical Facilities- } \\
\text { Related Functions }\end{array}$ & 0 & 4 & 2 & 0 & 0 & 6 \\
\hline $\begin{array}{l}\text { VI. Community- and } \\
\text { Alumni-Related } \\
\text { Functions } \\
\end{array}$ & 6 & 0 & 0 & 0 & 0 & 6 \\
\hline $\begin{array}{l}\text { VII. Spiritual Life-Related } \\
\text { Functions }\end{array}$ & 2 & 3 & 0 & 0 & 0 & 5 \\
\hline Totals & 15 & 21 & 10 & 1 & 0 & 47 \\
\hline Percentages & 31.9 & 44.7 & 21.3 & 2.1 & 0.0 & 100.0 \\
\hline
\end{tabular}


Of the total 47 role responsibilities expected of the presidents by the five status groups, Table 28 showed that, based upon the combined means of these respondents, one activity fell within the low expectation range (2.1\%). This item, "Know every student personally" ( $M=2.41, S D=1.32)$, was viewed with disagreement by all groups, though with high variances among them, that it should be the presidents' expected role responsibility. Ten expected roles came under moderate expectation category $(21.3 \%)$. Twenty-one expected role items were found to be in the high expectation category (44.7\%). Fifteen items fell under the very high expectation section (31.9\%), compared to two actual role items (4.3\%) that were found to have been actually performed very strongly. All six items (100\%) of community- and alumni-related functions were expected by the respondents to have a very strong presidential leadership. This indicates that all groups strongly agreed regarding these six role items that presidents should carry out these responsibilities with greater emphasis.

\section{Research Questions 3, 4, and 5 with Related Null Hypotheses 1, 2, and 3, Respectively}

Although the actual and the expected roles have been identified and although the overall four levels of perceptions and four levels of expectations have been determined by the mean scores, the differences between the five groups on the actual and the expected roles, and also the differences between the 47 actual roles and the 47 expected roles remain to be examined. Thus, the testing of the three null hypotheses was required to accomplish these purposes.

The results of the tests of the three null hypotheses are presented in this section. 
This section covers the data analysis associated with determining the differences, if any, among the means on actual performances of each of the 47 activities as viewed by five status groups. Therefore, there were 47 items tested in this section.

Data were tabulated by computer using the SPSS, and an Analysis of Variance was performed on the responses to the 47 items of the questionnaire to determine if a significant difference in perception existed among each of the five groups of respondents. Appendix $\mathrm{G}$ presents the composite results for this null hypothesis. Each ANOVA table includes mean $(M)$, standard deviation $(S D)$, and sample size $(N)$ for responses from the five categories of respondents, who responded to 47 questions on actual role items. Tables 33-39 also include overall statistics in these categories for the five groups in the study.

Purpose 3: Purpose 3 of this study was to determine the differences in actual and expected roles of presidents of Seventh-day Adventist senior colleges and universities around the world. Research Questions 3, 4, and 5 corresponded to this purpose.

Research Question 3 stated: Is there a difference in perception between groups of board chairs, presidents, academic vice presidents, student service vice presidents, and finance vice presidents of Seventh-day Adventist senior colleges and universities around the world regarding their senior college/university presidents' perceived actual role performances? This question was answered with Null Hypothesis 1.

\section{Null Hypothesis 1}

Null Hypothesis 1 stated that there is no difference among the five status groups 
(board chairs, presidents, academic vice presidents, student service vice presidents, and finance vice presidents) on each of the perceived actual role performances of presidents of Seventh-day Adventist senior colleges and universities around the world.

To test this null hypothesis, one-way analysis of variance was employed. The level of significance was set at 0.05 . However, to control for inflated Type 1 Error across the multiple ANOVAs, since there were many multiple comparisons, a corrected level of significance following the traditional Bonferroni method was used. ${ }^{1}$ There were 47 related variables on which five groups were compared on each variable. Therefore, the corrected level of significance was $0.05 / 47$, which was approximately $0.001 .^{2}$

Tables 33-39 in Appendix G present the item means and standard deviations by groups (board chairs, presidents, academic vice presidents, student service vice presidents, and finance vice presidents) of all 47 actual role performances by Seventh-day Adventist senior college/university presidents. Analysis of variance results of actual roles are also shown in Tables 47-53 in Appendix I. All $p$ values were greater than the corrected significance level at 0.001 . At alpha $=0.001$, no statistically significant group differences were found for all 47 actual role performance items. Null Hypothesis 1 for all 47 items was true. Therefore, Null Hypothesis 1 was retained.

Research Question 4 stated: Is there a difference in expectation between groups of board chairs, presidents, academic vice presidents, student service vice presidents, and

'Barbara G. Tabachnick and Linda S. Fidell, Using Multivariate Statistics (Boston, MA: Pearson Education, 2007), 402, 407.

${ }^{2}$ Samuel B. Green and Neil J. Salkind, Using SPSS for Windows and Macintosh: Analyzing and Understanding Data (Upper Saddle River, NJ: Prentice Hall, 2007), 204. 
finance vice presidents of Seventh-day Adventist senior colleges and universities around the world regarding their senior college/university presidents' expected role responsibilities?

\section{Null Hypothesis 2}

Null Hypothesis 2 stated that there is no difference among the five status groups (board chairs, presidents, academic vice presidents, student service vice presidents, and finance vice presidents) on each of the expected role responsibilities of presidents of Seventh-day Adventist senior colleges and universities around the world.

To test this hypothesis, one-way analysis of variance was employed. The level of significance was set at 0.05 . However, to control for inflated Type 1 Error across the multiple ANOVAs, since there were many multiple comparisons, a corrected level of significance following the traditional Bonferroni method was used. ${ }^{3}$ There were 47 related variables on which five groups were compared on each variable. Therefore, the corrected level of significance was $0.05 / 47$, which was approximately $0.001 .^{4}$

Tables 40-46 in Appendix $\mathrm{H}$ present the item means and standard deviations by groups (board chairs, presidents, academic vice presidents, student service vice presidents, and finance vice presidents) of all 47 expected role responsibilities of Seventh-day Adventist college/university presidents. Analysis of variance results of expected roles are also shown in Tables 47-53 in Appendix I. All $p$ values were greater than the corrected significance level at 0.001 . At alpha $=0.001$, no statistically

\footnotetext{
${ }^{3}$ Tabachnick, 402, 407.

${ }^{4}$ Green, 204.
} 
significant group differences were found for all 47 expected role items. Null Hypothesis 2 for all 47 items was true. Therefore, Null Hypothesis 2 was retained.

Research Question 5 stated: Is there a difference in perception and expectation between each of the actual role performances and expected role responsibilities of presidents of Seventh-day Adventist senior colleges and universities around the world, as perceived and expected by their groups of board chairs, presidents, academic vice presidents, student service vice presidents, and finance vice presidents?

\section{Null Hypothesis 3}

Null Hypothesis 3 stated that there is no difference between the perceived actual role performances and expected role responsibilities of presidents of Seventh-day Adventist senior colleges and universities around the world, as viewed by each of the status groups (board chairs, presidents, academic vice presidents, student service vice presidents, and finance vice presidents).

The purpose of this hypothesis was to examine whether there were differences in perceptions and expectations by the respondent groups between the actual roles and the expected roles. A two-tailed paired samples $t$-test was chosen to test this hypothesis and to see if the observed differences between the two means were the result of chance or they represented true differences. ${ }^{5}$ The SPSS statistical software was run that yielded results from the data obtained from the 78 subjects who had responded to the 47 pairs of

${ }^{5} R$. J. Shavelson, Statistical Reasoning for the Behavioral Sciences, $2^{\text {nd }}$ ed. (Boston, MA: Allyn and Bacon, 1988), quoted in Robert Alan Darden, "Study of the Efficacy of a Christian-Based Inpatient Hospital for Treating Eating Disorders, Depression, and Spiritual Distress" (Ph.D. dissertation, Andrews University, 2002), 77. 
questions on presidents' actual role performances compared to their expected role responsibilities. The mean differences for these 47 pairs were negative values, since the expected means, which were higher, were subtracted from the actual means, which were lower in each case. Because the mean differences were negative, the $t$ values were also negative. The $t$-test includes paired samples means $(M)$ and standard deviations $(S D)$-one for actual and one for expected. The $t$-test also includes mean deviations or mean differences $(M D), t$ values, degrees of freedom $(d f)$, and 2-tailed levels of significance $(P)$. The results of the $47 t$-tests between actual and expected roles are presented in Table 29. The discussion over the next several pages will elaborate on this Table 29.

The SPSS for Windows, version 12, was used for two-tailed paired samples $t$-tests to test for significant statistical differences between mean scores of actual performances and expected role responsibilities. The level of significance was set at alpha $=0.05$. However, to control for Type I Error since there were many multiple comparisons, a corrected level of significance following the Benferroni method was used. The corrected level of significance was $0.05 / 47$, which was approximately 0.001 .

Thirty-one out of the 47 pairs of actual roles and expected roles in Table 29 showed significant statistical differences between the actual and the expected means at alpha $\leq 0.001$ level of significance. Although the expected means were higher in every instance, the pairwise comparisons indicated that there were significant statistical differences between 31 actual and expected roles. Therefore, Null Hypothesis 3 for these 31 paired items was rejected, while the remaining 16 paired items of this hypothesis were retained. 
Table 29. Composite two-tailed $t$-test results comparing presidents' actual role performances and expected role responsibilities

\begin{tabular}{|c|c|c|c|c|c|c|}
\hline \multirow{3}{*}{ Item } & Actual (A) & Expected (E) & & & & \\
\hline & $N=78$ & $N=78$ & & & & $p$ \\
\hline & $S D$ & $S D$ & $M D$ & $t$ & $d f$ & (2-tailed) \\
\hline
\end{tabular}

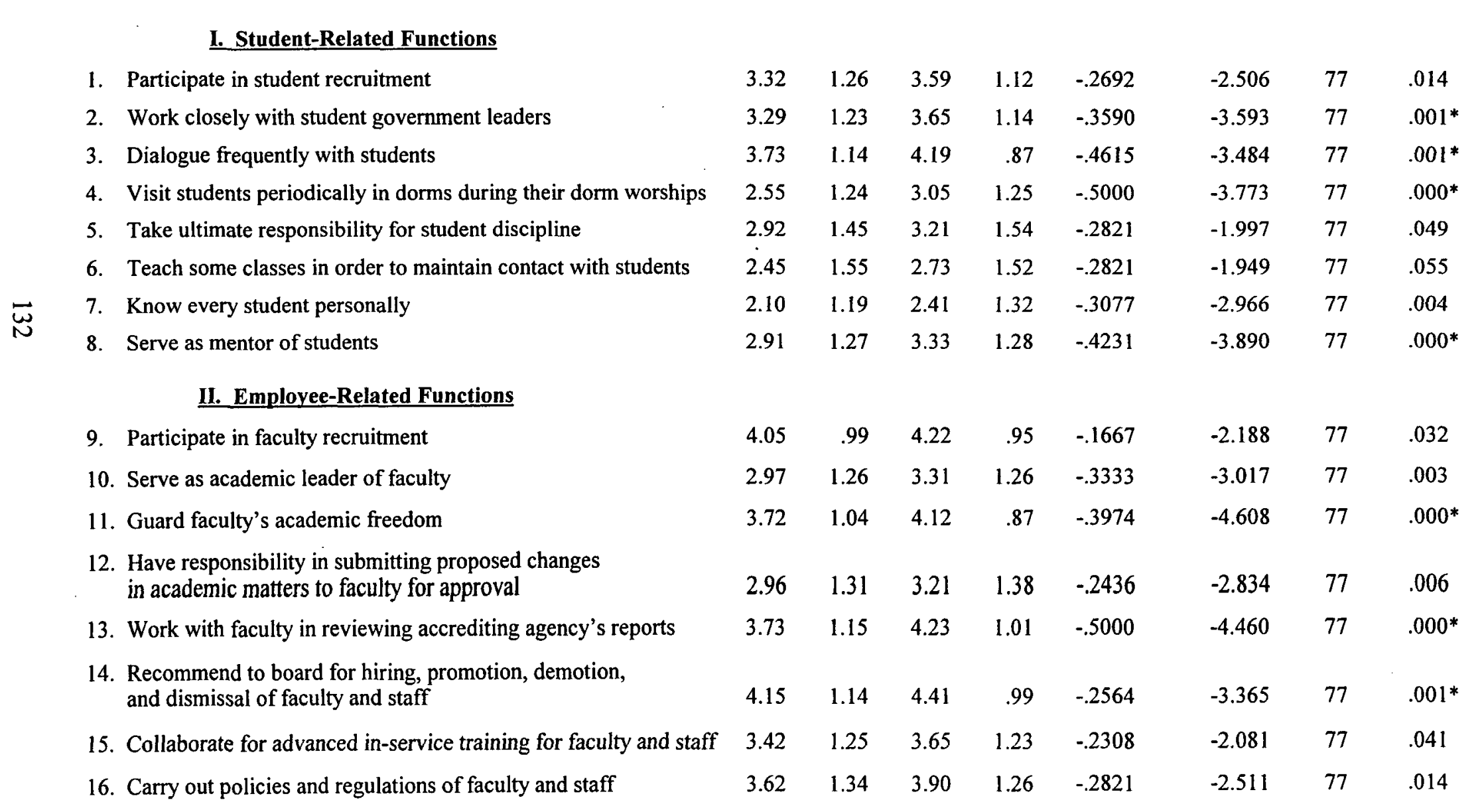


Table 29-Continued.

\begin{tabular}{|c|c|c|c|c|c|c|}
\hline \multirow{3}{*}{ Item } & Actual (A) & Expected (E) & & & & \multirow{3}{*}{$\underset{(2-t a i l e d)}{p}$} \\
\hline & $N=78$ & $N=78$ & & & & \\
\hline & $S D$ & $S D$ & $M D$ & $t$ & $d f$ & \\
\hline
\end{tabular}

\section{General Administrative Functions}

17. Prepare agenda for board of trustees' meetings

18. Preside over general faculty meetings

19. Take responsibility in structuring student admission standards

20. Take responsibility in strategic planning for institution

4.64

3.99

2.97

4.29

21. Take leading role to prepare, revise, \& follow organizational chart 4.04

22. Foster research and intellectual climate for institution

3.81

23. Comply with legal mandates and laws in addressing grievances $\quad 4.26$

24. Help formulate and adhere to institution's educational philosophy 4.44

.

.79

1.32

1.21

1.05

1.11

1.13

.93

1.14

\section{Finance-Related Functions}

26. Participate in the preparation of annual budget of institution

3.92

27. Ensure that institution's budget, approved by board, is adhered to 3.73

28. Present institution's annual audited financial statements to board 3.12

29. Recommend to board the salary changes for employee positions 3.28

30. Accept accountability to board for financial operation

4.05

1.29

1.20

1.47

1.42

1.14

4.81

4.03

3.27

4.76

4.53

4.27

4.60

4.87

4.73

.60

.60
1.35

$-.1667$

$-.0385$

$-2.493$

$-.418$

$\begin{array}{ll}1.26 & -.2949\end{array}$

$-3.697$

$-3.871$

$-4.529$

$-4.015$

$-4.260$

$-4.319$

$-4.551$

77

77
77

.015

.677

$\begin{array}{ll}.61 & -.4615 \\ 75 & -.4872\end{array}$

$\begin{array}{ll}.75 & -.4872\end{array}$

$.83--.4615$

$\begin{array}{ll}.74 & -.3462 \\ .37 & -.4359\end{array}$

$\begin{array}{ll}.53 & -.5897\end{array}$

77

77

77

$.000^{*}$

$.000^{*}$

$.000^{*}$

$.000^{*}$

$.000^{*}$

$.000^{*}$

$.000 *$

\section{Physical Facilities-Related Functions}

31. Present to board the physical needs of institution

4.06

32. Direct the preparation of plans for new buildings \& installations $\quad 3.50$

33. Plan major repairs or alterations of plants, buildings \& equipment 3.05

34. Recommend to board for equipment acquisitions

3.05

35. Ensure that physical plant master plans are current and followed 3.63

36. Ascertain that campus grounds are well-kept 
Table 29--Continued.

\begin{tabular}{|c|c|c|c|c|c|c|c|}
\hline \multirow[t]{2}{*}{ Item } & \multicolumn{2}{|c|}{$\begin{array}{c}\text { Actual (A) } \\
N=78\end{array}$} & \multicolumn{2}{|c|}{$\frac{\text { Expected (E) }}{N=78}$} & \multirow[b]{2}{*}{$M D$} & \multirow[b]{2}{*}{$t$} & \multirow[b]{2}{*}{ (2-tailed) } \\
\hline & $M$ & $S D$ & $M$ & $S D$ & & & \\
\hline
\end{tabular}

\section{Community and Alumni-Related Functions}

37. Assist in raising funds for support of the institution

38. Represent institution to churches of the constituency

$\begin{array}{rrrrrrrr}4.09 & 1.15 & 4.77 & .64 & -.6795 & -5.712 & 77 & .000^{*} \\ 4.40 & .92 & 4.85 & .40 & -.4487 & -4.855 & 77 & .000^{*} \\ 4.28 & 1.07 & 4.67 & .64 & -.3846 & -4.196 & 77 & .000^{*} \\ 4.49 & .88 & 4.76 & .71 & -.2692 & -3.613 & 77 & .001^{*} \\ 4.05 & 1.01 & 4.51 & .62 & -.4615 & -4.357 & 77 & .000^{*} \\ 4.23 & 1.04 & 4.74 & .47 & -.5128 & -4.458 & 77 & .000^{*}\end{array}$

39. Present reports about institution to community and alumni

40. Represent institution as its official spokesperson to public

41. Associate closely with alumni groups

42. Listen to the constituency and alumni

\section{Spiritual Life-Related Functions}

43. Assume primary responsibility for promotion of students' religious life at the institution via delegation

$\begin{array}{llllllll}4.08 & 1.10 & 4.47 & .92 & -.3974 & -3.613 & 77 & .001^{*} \\ 3.77 & 1.19 & 4.35 & .99 & -.5769 & -5.461 & 77 & .000^{*} \\ & & & & & & & \\ 3.35 & 1.34 & 3.91 & 1.23 & -.5641 & -5.256 & 77 & .000^{*} \\ 4.46 & .80 & 4.83 & .44 & -.3718 & -3.913 & 77 & .000^{*} \\ 4.64 & .77 & 4.88 & .32 & -.2436 & -2.772 & 77 & .007\end{array}$

44. Assume primary responsibility for promotion of religious life of faculty and staff at the institution via delegation

45. Take ultimate responsibility for planning midweek chapels, vespers, weeks of spiritual emphasis and other religious programs on campus via delegation for spiritual nurture

46. Manifest Christ-like character in spiritual leadership roles

47. Focus on Christian philosophy of institution

*Denotes pairs of actual and expected roles significantly different at $p \leq 0.001$. 
The mean scores for actual roles compared to mean scores for expected roles showed mean differences $(M D), t$ values, and 2-tailed levels of significance, suggesting a statistical increase in each of the expected role items. Further, Table 29 shows 16 cases having no significant statistical differences in perceptions and expectations between actual role performances and expected role responsibilities of presidents as viewed by the groups of respondents.

\section{Student-Related Functions}

Item 1, "Participate in student recruitment," had an actual group mean $M=3.32$ with a standard deviation $S D=1.26$ compared to an expected group mean $M=3.59$ with a standard deviation $S D=1.12$, showing a mean difference $M D=-0.2692$ between actual and expected. However, the slight increase in expected mean score from actual mean score made no statistical difference at alpha $=0.001$ or less between this actual and expected role item. The $t$-test comparing actual and expected means yielded a $t_{1,77}=-2.506$, and $p=0.014$. Thus, Null Hypothesis 3 for Item 1 was retained.

Item 2, "Work closely with student government leaders," had an actual group mean $M=3.29$ with a standard deviation $S D=1.23$ compared to an expected group mean $M=3.65$ with a standard deviation $S D=1.14$, showing a mean difference $M D=-0.3590$. This high increase in expected mean from actual mean made a significant statistical difference at alpha $=0.001$ or less between this actual and expected role item. The $t$-test comparing actual and expected means yielded a $t_{1,77}=-3.593$ and $p=0.001$. Thus, Null Hypothesis 3 for Item 2 was rejected. 
Item 3, "Dialogue frequently with students," had an actual group mean $M=3.73$ with a standard deviation $S D=1.14$ compared to an expected group mean $M=4.19$ with a standard deviation $S D=0.87$, showing a mean difference $M D=-0.4615$. This high increase in expected mean from actual mean made a significant statistical difference at alpha $=0.001$ or less between this actual and expected role item. The $t$-test comparing actual and expected means yielded a $t_{1,77}=-3.484$ and $p=0.001$. Thus, Null Hypothesis 3 for Item 3 was rejected.

Item 4, "Visit students periodically in dorms during their dorm worships," had an actual mean $M=2.55$ with a standard deviation $S D=1.24$ compared to an expected mean $M=3.05$ with a standard deviation $S D=1.25$, showing a mean difference $M D=-0.5000$ between actual and expected. This significant increase in expected mean from actual mean made a significant statistical difference at alpha $=0.001$ or less between this actual and expected role item. The $t$-test comparing actual and expected means yielded a $t_{1,77}=-3.773$ and $p=0.000$. Thus, Null Hypothesis 3 for Item 4 was rejected.

Item 5, "Take ultimate responsibility for student discipline," had an actual group mean $M=2.92$ with a standard deviation $S D=1.45$ compared to an expected group mean $M=3.21$ with a standard deviation $S D=1.54$, showing a mean difference $M D=-0.2821$ between actual and expected. However, this slight increase in expected mean score from actual mean score made no significant statistical difference at alpha $=0.001$ or less between this actual and expected role item. The $t$-test comparing actual and expected means yielded a $t_{1,77}=-1.997$ and $p=0.049$. Thus, Null Hypothesis 3 for Item 5 was retained. 
Item 6, "Teach some classes to maintain contact with students," had an actual mean $M=2.45$ with a standard deviation $S D=1.55$ compared to an expected mean $M=2.73$ with a standard deviation $S D=1.52$, showing a mean difference $M D=-0.2821$ between actual and expected. However, the slight increase in expected mean from actual mean made no statistical difference at alpha $=0.001$ or less between this actual and expected role item. The $t$-test comparing actual and expected means yielded a $t_{1,77}=-1.949$ and $p=0.055$. Thus, Null Hypothesis 3 for Item 6 was retained.

Item 7, "Know every student personally," had an actual group mean $M=2.10$ with a standard deviation $S D=1.19$ compared to an expected group mean $M=2.41$ with a standard deviation $S D=1.32$, showing a mean difference $M D=-0.3077$ between actual and expected. Although there was a big increase in expected mean score from actual mean score, no significant statistical difference was found between this actual and expected role item at alpha $=0.001$ or less. The $t$-test comparing actual and expected means yielded a $t_{1,77}=-2.966$ and $p=0.004$. Thus, Null Hypothesis 3 for Item 7 was retained.

Item 8, "Serve as mentor of students," had an actual group mean $M=2.91$ with a standard deviation $S D=1.27$ compared to an expected group mean $M=3.33$ with a standard deviation $S D=1.28$, showing a mean difference $M D=-0.4231$ between actual and expected. This big increase in expected mean from actual mean made a significant statistical difference at alpha $=0.001$ or less between this actual and expected role item. The $t$-test comparing actual and expected means yielded a $t_{1,77}=-3.890$ and $p=0.000$. Thus, Null Hypothesis 3 for Item 8 was rejected. 


\section{Employee-Related Functions}

Item 9, "Participate in faculty recruitment," had an actual group mean $M=4.05$ with a standard deviation $S D=0.99$ compared to an expected group mean $M=4.22$ with a standard deviation $S D=0.95$, showing a mean difference $M D=-0.1667$ between actual and expected. However, this slight increase in expected mean score from actual mean score made no statistical difference at alpha $=0.001$ or less between this actual and expected role item. The $t$-test comparing actual and expected means yielded a $t_{1,77}=-2.188$ and $p=0.032$. Thus, Null Hypothesis 3 for Item 9 was retained.

Item 10, "Serve as academic leader of faculty," had an actual group mean $M=2.97$ with a standard deviation $S D=1.26$ compared to an expected group mean $M=3.31$ with a standard deviation $S D=1.26$, showing a mean difference $M D=-0.3333$ between actual and expected. There was a notable increase in expected mean score from actual mean score, but it made no statistical difference at alpha $=0.001$ or less between this actual and expected role item. The $t$-test comparing actual and expected means yielded a $t_{1,77}=-3.017$ and $p=0.003$. Thus, Null Hypothesis 3 for Item 10 was retained.

Item 11, "Guard faculty's academic freedom," had an actual group mean $M=3.72$ with a standard deviation $S D=1.04$ compared to an expected group mean $M=4.12$ with a standard deviation $S D=0.87$, showing a big mean difference $M D=-0.3974$ between actual and expected. This big increase in expected mean from actual mean made a significant statistical difference at alpha $=0.001$ or less between this actual and expected role item. The $t$-test comparing actual and expected means yielded a $t_{1,77}=-4.608$ and $p=0.000$. Thus, Null Hypothesis 3 for Item 11 was rejected. 
Item 12, "Have responsibility in submitting proposed changes in academic matters to faculty for approval," had an actual group mean $M=2.96$ with a standard deviation $S D=1.31$ compared to expected group mean score $M=3.21$ with a standard deviation $S D=1.38$, showing a mean difference $M D=-0.2436$. However, this slight increase in expected mean score from actual mean score made no statistical difference at alpha $=0.001$ or less between this actual and expected role item. The $t$-test comparing actual and expected means yielded a $t_{1,77}=-2.834$ and $p=0.006$. Thus, Null Hypothesis 3 for Item 12 was retained.

Item 13, "Work with faculty in reviewing accrediting agency's reports," had an actual group mean $M=3.73$ with a standard deviation $S D=1.15$ compared to an expected group mean $M=4.23$ with a standard deviation $S D=1.01$, showing a mean difference $M D=-0.5000$. The high increase in expected mean from actual mean made a significant statistical difference at alpha $=0.001$ or less between this actual and expected role item. The $t$-test comparing actual and expected means yielded a $t_{1,77}=-4.460$ and $p=0.000$. Thus, Null Hypothesis 3 for Item 13 was rejected.

Item 14, "Recommend to board for hiring, promotion, demotion, and dismissal of faculty and staff," had an actual group mean $M=4.15$ with a standard deviation $S D=1.14$ compared to an expected group mean $M=4.41$ with a standard deviation $S D=0.99$, showing a mean difference $M D=-0.2564$. Although there was a slight increase in expected mean score from actual mean score, a significant statistical difference was found between this actual and expected role item at alpha $=0.001$ or less. The $t$-test comparing actual and expected means yielded a $t_{1,77}=-3.365$ and $p=0.001$. 
Thus, Null Hypothesis 3 for Item 14 was rejected.

Item 15 , "Collaborate for advanced in-service training for faculty and staff," had an actual group mean $M=3.42$ with a standard deviation $S D=1.25$ compared to an expected group mean $M=3.65$ with a standard deviation $S D=1.23$, showing a small mean difference $M D=-0.2308$. However, this slight increase in expected mean from actual mean made no statistical difference at alpha $=0.001$ or less between this actual and expected role item. The $t$-test comparing actual and expected means yielded a $t_{1,77}=-2.081$ and $p=0.041$. Thus, Null Hypothesis 3 for Item 15 was retained.

Item 16, "Carry out policies and regulations pertaining to faculty and staff," had an actual group mean $M=3.62$ with a standard deviation $S D=1.34$ compared to an expected group mean $M=3.90$ with a standard deviation $S D=1.26$, showing a mean difference $M D=-0.2821$. However, this slight increase in expected mean from actual mean made no statistical difference at alpha $=0.001$ or less between this actual and expected role item. The $t$-test comparing actual and expected means yielded a $t_{1,77}=-2.511$ and $p=0.014$. Thus, Null Hypothesis 3 for Item 16 was retained.

\section{General Administrative Functions}

Item 17, "Prepare agenda for board of trustees' meetings," had an actual group mean $M=4.64$ with a standard deviation $S D=0.79$ compared to an expected group mean $M=4.81$ with a standard deviation $S D=0.60$, showing a mean difference $M D=-0.1667$. However, this slight increase in expected mean score from actual mean score made no statistical difference between this actual and expected role item at 
alpha $=0.001$ or less. The $t$-test comparing actual and expected means yielded a $t_{1,77}=-2.493$ and $p=0.015$. Thus, Null Hypothesis 3 for Item 17 was retained.

Item 18 , "Preside over general faculty meetings," had an actual group mean $M=3.99$ with a standard deviation $S D=1.32$ compared to an expected group mean $M=4.03$ with a standard deviation $S D=1.35$, showing a mean difference $M D=-0.0385$. This very small increase in expected mean score from actual mean score made no statistical difference between this actual and expected role item at alpha $=0.001$ or less. The $t$-test comparing actual and expected means yielded a $t_{1,77}=-0.418$ and $p=0.677$. Thus, Null Hypothesis 3 for Item 18 was retained.

The next seven items of general administrative functions had their expected group means higher than their actual group means in each case. These items are as follows:

Item 19, "Take responsibility in structuring student admission standards," yielded a $t_{1,77}=-3.697$ and $p=0.000$.

Item 20, "Take responsibility in strategic planning for the institution," yielded a $t_{1,77}=-3.871$ and $p=0.000$.

Item 21 , "Take a leading role to prepare, revise, and follow organizational chart," yielded a $t_{1,77}=-4.529$ and $p=0.000$.

Item 22, "Foster research and intellectual climate for the institution," yielded a $t_{1,77}=-4.015$ and $p=0.000$.

Item 23 , "Comply with legal mandates and laws in addressing grievances," yielded a $t_{1,77}=-4.260$ and $p=0.000$.

Item 24, "Help formulate and adhere to institution's educational philosophy," 
yielded a $t_{1,77}=-4.319$ and $p=0.000$.

Item 25, "Delegate responsibility, authority, and needed resources to appropriate administrators and support their roles," yielded a $t_{1,77}=-4.551$ and $p=0.000$.

Each of these items showed significant statistical differences at alpha $=0.001$ or less between actual and expected roles. The $t$-tests comparing actual and expected means for these items showed their $p$ values at alpha $=0.001$ and less. Thus, Null Hypothesis 3 for Items 19 through 25 was rejected.

\section{Finance-Related Functions}

All five items of finance-related functions had their expected group means higher than their actual group means in each case. These items are as follows:

Item 26, "Participate in the preparation of annual operating budget of the institution," yielded a $t_{1,77}=-3.803$ and $p=0.000$.

Item 27, "Ensure that institution's operating budget, after approval by board, is adhered to," yielded a $t_{1,77}=-6.007$ and $p=0.000$.

Item 28, "Present annual audited financial statements to board," yielded a $t_{1,77}=-3.326$ and $p=0.001$.

Item 29 , "Recommend to board the salary changes for faculty/staff positions," yielded a $t_{1,77}=-4.292$ and $p=0.000$.

Item 30, "Accept accountability to board for institution's financial operation," yielded a $t_{1,77}=-5.500$ and $p=0.000$.

Each of these items showed that the $t$-tests comparing actual and expected means 
were statistically significant at alpha $=0.001$ or less. Thus, Null Hypothesis 3 for Items 26 through 30 was rejected.

\section{Physical Facilities-Related Functions}

Item 31 , "Present to the board the physical needs of the institution," had an actual group mean $M=4.06$ with a standard deviation $S D=1.24$ compared to an expected group mean $M=4.33$ with a standard deviation $S D=1.16$, showing a mean difference $M D=-0.2692$. However, this slight increase in expected mean from actual mean made no statistical difference between this actual and expected role item at alpha $=0.001$ or less. The $t$-test comparing actual and expected means yielded a $t_{1,77}=-2.857$ and $p=0.006$. Thus, Null Hypothesis 3 for Item 31 was retained.

Item 32, "Direct the preparation of plans for new buildings and installations as needed," had an actual group mean $M=3.50$ with a standard deviation $S D=1.44$ compared to an expected group mean $M=3.79$ with a standard deviation $S D=1.22$, showing a mean difference $M D=-0.2949$. There is a high increase in expected mean from actual mean, but it showed no statistical difference between this actual and expected role item at alpha $=0.001$ or less. The $t$-test comparing actual and expected means yielded a $t_{1,77}=-2.727$ and $p=0.008$. Thus, Null Hypothesis 3 for Item 32 was retained.

Item 33, "Plan for major repairs or alterations of plant, buildings, and equipment," had an actual group mean $M=3.05$ with a standard deviation $S D=1.39$ compared to an expected group mean $M=3.17$ with a standard deviation of $S D=1.34$, showing a small mean difference $M D=-0.1154$. However, this slight increase in expected mean from 
actual mean made no statistical difference between this actual and expected role item at alpha $=0.001$ or less. The $t$-test comparing actual and expected means yielded a $t_{1,77}=-1.136$ and $p=0.259$. Thus, Null Hypothesis 3 for Item 33 was retained.

Item 34, "Recommend to the board for equipment acquisitions," had an actual group mean $M=3.05$ with a standard deviation $S D=1.50$ compared to an expected group mean $M=3.36$ with a standard deviation $S D=1.55$, showing a mean difference $M D=-0.3077$. Although it did not show a high increase in expected mean from actual mean, a significant statistical difference was found at alpha $=0.001$ or less. The $t$-test comparing actual and expected means yielded a $t_{1,77}=-3.653$ and $p=0.000$. Thus, Null Hypothesis 3 for Item 34 was rejected.

Item 35, "Ensure that physical plant master plans are current and are followed as closely as possible," had an actual group mean $M=3.63$ with a standard deviation $S D=1.36$ compared to an expected group mean $M=4.09$ with a standard deviation $S D=1.08$, showing a mean difference $M D=-0.4615$. There was a notable increase in expected mean from actual mean that made a significant statistical difference at alpha $=0.001$ or less. The $t$-test comparing actual and expected means yielded a $t_{1,77}=-3.588$ and $p=0.001$. Thus, Null Hypothesis 3 for Item 35 was rejected.

Item 36, "Ascertain that campus grounds are well-kept through appropriate officer and administrator," had an actual group mean $M=3.73$ with a standard deviation $S D=1.18$ compared to an expected group mean $M=4.04$ with a standard deviation $S D=1.12$, showing a mean difference $M D=-0.3077$. However, this increase in expected mean from actual mean made no statistical difference at alpha $=0.001$ or less. 
The $t$-test comparing actual and expected means yielded a $t_{1,77}=-3.013$ and $p=0.003$. Thus, Null Hypothesis 3 for Item 36 was retained.

\section{Community- and Alumni-Related Functions}

All six items of community- and alumni-related functions had their expected group means higher than their actual group means in each case. These items were:

Item 37, "Assist in raising funds for support of institution," yielded a $t_{1,77}=-5.712$ and $p=0.000$.

Item 38, "Represent institution to churches of the constituency," yielded a $t_{1,77}=-4.855$ and $p=0.000$.

Item 39, "Present reports about institution to community and alumni," yielded a $t_{1,77}=-4.196$ and $p=0.000$.

Item 40, "Represent institution as its official spokesperson to public," yielded a $t_{1,77}=-3.613$ and $p=0.001$.

Item 41, "Associate closely with alumni groups," yielded a $t_{1,77}=-4.357$ and $p=0.000$

Item 42 , "Listen to constituency and alumni," yielded a $t_{1,77}=-4.458$ and $p=0.000$.

Each of these above items showed significant statistical differences at alpha $=0.001$ or less between actual and expected roles. The $t$-tests comparing actual and expected means for these items showed their $p$ values at alpha $=0.001$ and less. Thus, Null Hypothesis 3 for Items 37 through 42 was rejected. 


\section{Spiritual Life-Related Functions}

Four spiritual life-related items had their expected group means higher than their actual group means in each case. These items were as follows:

Item 43 , "Assume primary responsibility for promotion of students' religious life at the institution via delegation," yielded a $t_{1,77}=-3.613$ and $p=0.001$.

Item 44, “Assume primary responsibility for promotion of religious life of faculty and staff at the institution via delegation," yielded a $t_{1,77}=-5.461$ and $p=0.000$.

Item 45 , "Take ultimate responsibility for planning midweek chapels, vespers, weeks of spiritual emphasis, and other religious programs on campus via delegation for spiritual nurture," yielded a $t_{1,77}=-5.256$ and $p=0.000$.

Item 46, "Manifest Christ-like character in spiritual leadership roles," yielded a $t_{1,77}=-3.913$ and $p=0.000$.

Each of these above items showed significant statistical differences at alpha $=0.001$ or less between actual and expected roles. The $t$-tests comparing actual and expected means for these items showed their $p$ values at alpha $=0.001$ and less. Thus, Null Hypothesis 3 for Items 43 through 46 was rejected.

Item 47, "Focus on Christian philosophy of institution," had an actual group mean $M=4.64$ with a standard deviation $S D=0.77$ compared to an expected group mean $M=4.88$ with a standard deviation $S D=0.32$, showing a mean difference $M D=-0.2436$. However, this slight increase in expected mean from actual mean made no significant statistical difference at alpha $=0.001$ or less between this actual and expected role item. The $t$-test comparing actual and expected means yielded a $t_{1,77}=-2.772$ and $p=0.007$. 
Thus, Null Hypothesis 3 for Item 47 was retained.

Table 30 is a summary of results drawn from Table 26 and Table 28 on levels of actual and expected roles, respectively. Table 30 provides an overall comparison in percentage between the presidents' actual role performances in 2004-2005 and their expected role responsibilities as viewed by the five status groups. This table shows that the presidents' overall roles are expected to be higher than their actual role performances.

While $4.3 \%$ of the overall actual role performances were perceived to be very strong in the $2004-2005$ school year, $31.9 \%$ (up by $27.6 \%$ ) of those roles were expected to be of very high responsibility of the presidents. While $59.5 \%$ of the actual roles were considered as strong performance, $44.7 \%$ (less by $14.8 \%$ ) of those roles should be in the high expectation category according to the status groups. Expected roles were less by $14.8 \%$ than actual roles in the strong expectation category, because there was an increase in expected roles by $27.6 \%$ in the very high expectation category. While $31.9 \%$ of the overall actual roles were perceived to be of moderate performance in 2004-2005, a percentage of 21.3 (less by 10.6\%) of those roles was expected to be of moderate responsibility. While $4.3 \%$ of the actual roles were perceived to be of weak performance in $2004-2005$, only $2.1 \%$ (less by $2.2 \%$ ) of those roles were expected to be categorized as low responsibility items.

Table 54 in Appendix $\mathrm{J}$ contains 47 roles in their various levels of performances and expectations from highest to lowest means. The diagonal lines show the elevated positions of expected roles compared to actual roles performed. While "Focus on Christian philosophy" in the highest category came on top for both actual practice and 
expected role responsibility, "Know every student personally," in the lowest category was perceived as weak performance and low expectation. "Prepare board meeting agendas" in the first classification shows a downward line from actual to expected, but expected mean is higher and standard deviation is lower than actual. Table 55 in Appendix $\mathrm{K}$ is a re-arrangement of four levels of actual and expected roles by categories of functions, based on Tables 25 and 27.

Table 30. Comparisons by percentages between actual and expected roles, based upon means

\begin{tabular}{|c|c|c|c|c|c|}
\hline \multirow{5}{*}{ Role } & \multicolumn{5}{|c|}{ Levels of Perceptions of Actual Versus Expected Roles } \\
\hline & Level 1 & Level 2 & Level 3 & Level 4 & $\underline{\text { Level } 5}$ \\
\hline & $\begin{array}{l}\text { Very Strong } \\
\text { Role } \\
\text { Performance }\end{array}$ & $\begin{array}{l}\text { Strong } \\
\text { Role } \\
\text { Performance }\end{array}$ & $\begin{array}{l}\text { Moderate } \\
\text { Role } \\
\text { Performance }\end{array}$ & $\begin{array}{c}\text { Weak } \\
\text { Role } \\
\text { Performance }\end{array}$ & $\begin{array}{l}\text { Very Weak } \\
\text { Role } \\
\text { Performance }\end{array}$ \\
\hline & Level 1 & Level 2 & Level 3 & Level 4 & Level 5 \\
\hline & $\begin{array}{l}\text { Very High } \\
\text { Role } \\
\text { Expectation }\end{array}$ & $\begin{array}{c}\text { High } \\
\text { Role } \\
\text { Expectation }\end{array}$ & $\begin{array}{l}\text { Moderate } \\
\text { Role } \\
\text { Expectation }\end{array}$ & $\begin{array}{c}\text { Low } \\
\text { Role } \\
\text { Expectation }\end{array}$ & $\begin{array}{l}\text { Very Low } \\
\text { Role } \\
\text { Expectation }\end{array}$ \\
\hline Actual & $4.3 \%$ & $59.5 \%$ & $31.9 \%$ & $4.3 \%$ & $0.00 \%$ \\
\hline Expected & $31.9 \%$ & $44.7 \%$ & $21.3 \%$ & $2.1 \%$ & $0.00 \%$ \\
\hline
\end{tabular}

Table 31 presents a summary of statistical results of testing the three null hypotheses--Null Hypothesis 1, Null Hypothesis 2, and Null Hypothesis 3--that answered the three corresponding research questions--Research 3, Research Question 4, and Research Question 5, discussed in this chapter. 
Table 31. Summary results of null hypotheses tested

$$
\text { RQ3 RQ4 RQ5 }
$$

Item

Null Hypothesis

\begin{tabular}{lll}
\hline 1 & 2 & 3
\end{tabular}

\section{Student-Related Functions}

2. Work closely with student government leaders NS

NS

NS

3. Dialogue frequently with students NS NS

4. Visit students in dorms periodically during their dorm worships NS NS NS

5. Take ultimate responsibility for student discipline NS

6. Teach some classes in order to maintain contact with students NS

7. Know every student personally NS

8. Serve as mentor of students NS

\section{NS}

NS

NS

\section{Employee-Related Functions}

9. Participate in faculty recruitment NS

NS

10. Serve as academic leader of faculty NS

NS

NS

11. Guard faculty's academic freedom NS

12. Have responsibility in submitting proposed changes in academic matters to faculty for approval NS NS $*$

13. Work with faculty in reviewing accrediting agency's reports NS

14. Recommend to board for hiring, promotion, demotion and dismissal of faculty and staff. NS

15. Collaborate for advanced in-service training for faculty and staff NS

16. Carry out policies and regulations pertaining to faculty and staff NS

\section{General Administrative Functions}

17. Prepare agenda for board of trustees' meetings ..................................... NS

NS $\quad *$

18. Preside over general faculty meetings NS

19. Take responsibility in structuring student admission standards NS

20. Take responsibility in strategic planning for institution NS

21. Take a leading role to prepare, revise, and follow organizational chart NS

22. Foster research and intellectual climate for the institution..... NS

23. Comply with legal mandates \& laws in addressing employee grievances .... NS

24. Help formulate and adhere to institution's educational philosophy NS

25. Delegate responsibility, authority and needed resources to appropriate administrators and support their roles NS 
Table 31-Continued.

\begin{tabular}{|c|c|c|c|}
\hline \multirow{3}{*}{ Item } & RQ3 & RQ4 & RQ5 \\
\hline & \multicolumn{3}{|c|}{ Null Hypothesis } \\
\hline & 1 & 2 & 3 \\
\hline \multicolumn{4}{|l|}{ IV. Finance-Related Functions } \\
\hline 26. Participate in the preparation of annual budget of the institution ................... & NS & NS & * \\
\hline 27. Ensure that institution's budget, after approval by board, is adhered to ....... & NS & NS & $*$ \\
\hline 28. Present institution's annual audited financial statements to board ................... & NS & NS & * \\
\hline 29. Recommend to board the salary changes for faculty and staff positions ....... & NS & NS & * \\
\hline 30. Accept accountability to board for financial operation of institution ............. & NS & NS & $*$ \\
\hline \multicolumn{4}{|l|}{ V. Physical Facilities-Related Functions } \\
\hline 31. Present to board the physical needs of the institution & NS & NS & NS \\
\hline 32. Direct the preparation of plans for new buildings and installations ................. & NS & NS & NS \\
\hline 33. Plan for major repairs and alterations of plant, buildings, and equipment ... & NS & NS & NS \\
\hline 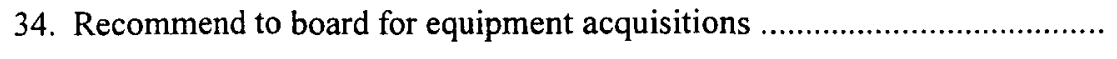 & NS & NS & $*$ \\
\hline 35. Ensure that physical plant master plans are current and followed closely ... & NS & NS & * \\
\hline $\begin{array}{l}\text { 36. Ascertain that campus grounds are well-kept through appropriate } \\
\text { officer and administrator }\end{array}$ & NS & NS & NS \\
\hline \multicolumn{4}{|l|}{ VI. Community-and Alumni-Related Functions } \\
\hline 37. Assist in raising funds for support of institution & NS & NS & * \\
\hline 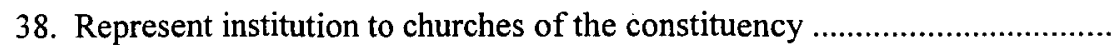 & NS & NS & * \\
\hline 39. Present reports about college/university to community and alumni ............. & NS & NS & * \\
\hline 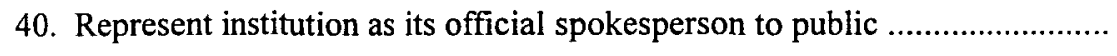 & NS & NS & * \\
\hline 41. Associate closely with alumni groups & NS & NS & * \\
\hline 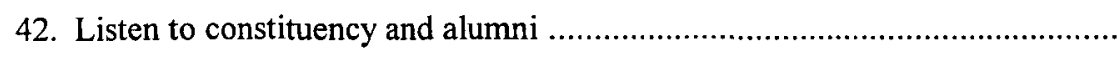 & NS & NS & * \\
\hline \multicolumn{4}{|l|}{ VII. Spiritual Life-Related Functions } \\
\hline $\begin{array}{l}\text { 43. Assume primary responsibility for promotion of students' } \\
\text { religious life at the institution via delegation }\end{array}$ & NS & NS & $*$ \\
\hline $\begin{array}{l}\text { 44. Assume primary responsibility for promotion of religious life of } \\
\text { faculty and staff at the institution via delegation }\end{array}$ & NS & NS & * \\
\hline $\begin{array}{l}\text { 45. Take ultimate responsibility for planning midweek chapels, vespers, } \\
\text { weeks of spiritual emphasis, and other religious programs on } \\
\text { campus via delegation for spiritual nurture }\end{array}$ & NS & NS & * \\
\hline 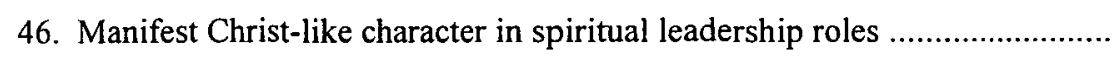 & NS & NS & * \\
\hline 47. Focus on Christian philosophy of institution & NS & NS & NS \\
\hline
\end{tabular}

Note: $\mathrm{RQ}=$ Research Question; NS = Not significant.

* Denotes statistical test significant at $p \leq .001$ or less. 


\section{Summary}

This chapter dealt with sample characteristics, data presentation, data analysis, and statistical results. A step-by-step procedure was followed to examine the purposes of the study, five research questions, and the three related null hypotheses under study. Descriptive statistics gave degrees of perceptions of status groups on actual role performance and expected role responsibilities of presidents as rank ordered by means. Five research questions were answered.

Research Question 1 was answered by descriptive statistics (Table 25) that identified perceptions of presidents' actual role performances in 2004-2005 in four levels, rank ordered by means from highest to lowest--very strong role performance, strong role performance, moderate role performance, and weak role performance. Only 2 role items out of a total of 47 role items were perceived to have been performed very strongly.

Research Question 2 was answered by descriptive statistics (Table 27) that identified perceptions of presidents' expected role responsibilities in four levels of expectations, rank ordered by means from highest to lowest--very high role expectation, high role expectation, moderate role expectation, and low role expectation. As many as 15 items out of a total of 47 items were expected to be of very high role responsibilities.

Research Questions 3, 4, and 5 were answered by three corresponding null hypotheses--Null Hypothesis 1, Null Hypothesis 2, and Null Hypothesis 3, respectively.

Null Hypothesis 1: There is no difference among the five status groups (board chairs, presidents, academic vice presidents, student service vice presidents, and finance vice presidents) on each of the actual role performances of the presidents. 
This null hypothesis was retained. There were no statistically significant differences of perceptions existed among the five groups of respondents on any of the presidents' 47 actual role performances in 2004-2005 at $p \leq 0.001$ using the traditional Bonferroni method that required this new corrected significance level in order to control for Type I Error. See Tables 33-39 in Appendix G and also Tables 47-53 in Appendix I.

Null Hypothesis 2: There is no difference among the five status groups (board chairs, presidents, academic vice presidents, student service vice presidents, and finance vice presidents) on each of the expected role responsibilities of the presidents.

This null hypothesis was retained. There were no statistically significant differences perceived to be existing among the five groups of respondents on any of the presidents' 47 expected role responsibilities at $p \leq 0.001$ using the traditional Bonferroni method that required this new corrected significance level in order to control for inflated Type I Error. See Tables 40-46 in Appendix H and also Tables 47-53 in Appendix I.

Null Hypothesis 3: There are no differences between the actual roles and the expected role responsibilities of presidents as viewed by each of the five status groups.

The $t$-test revealed that all of the 47 expected role means were higher than all of the 47 actual role means. The pairwise comparisons showed that 31 pairs out of the total of 47 pairs had significant statistical differences between the actual and expected roles at $p \leq 0.001$ following the Bonferroni approach. Therefore, Null Hypothesis 3 for these 31 pairs of actual and expected roles was rejected. The remaining 16 pairs showed no significant statistical differences between the actual and expected roles. Therefore, Null Hypothesis 3 for these 16 pairs of actual and expected roles was retained. See Table 29. 


\section{CHAPTER V}

\section{SUMMARY, FINDINGS, DISCUSSION, CONCLUSIONS, AND RECOMMENDATIONS}

\section{Introduction}

In this final chapter, I summarize the salient points of the dissertation. I begin with an overview of the background of the study and then move on to the problem, purpose of the study, and a brief review of literature. This chapter also presents the methodology used in addressing the five research questions and the three corresponding research hypotheses. It identifies the results obtained by testing the three null hypotheses; discusses the research findings; and provides reflective conclusions about the outcome of the study. The chapter closes with recommendations for practice and further research.

\section{Summary}

\section{Background of the Study}

Seventh-day Adventists are credited with one of the largest Protestant school systems in the world. They operate 28 senior colleges and universities around the world that offer graduate degree programs on their campuses under their own authorities, as per research survey done in the 2004-2005 academic year. The presidents, or vicechancellors, or chancellors, or rectors, or principals, as they are called in different 
countries, ${ }^{1}$ served as chief executive officers of their institutions and boards. The General Conference (GC) is the highest decision-making, policy-formulating, performanceevaluating, and the GC institution's CEO-appointing body for the Seventh-day Adventist world church in matters of all branches of activities, including those of education. The GC Education Department did not have, at the time of survey for this research, any welldefined job descriptions for the chief executive officers of the SDA senior colleges and universities. Earlier, this body had sent out to all the SDA higher education institutions around the world a list of topics for academic research, one of which was the role and function of Seventh-day Adventist college and university presidents. This study is the result of my acceptance of that challenge.

\section{The Problem}

The Seventh-day Adventist senior college and university presidents are called upon to perform many functions. Apparently, there is a need for adequate, clear-cut job descriptions of all the SDA senior college or university presidents around the world. Delegation of responsibilities by presidents among vice presidents and unit administrators is feared ambiguous. Role perceptions and role expectations of college/university presidents are subject to variance among the reference groups. As a result, there are chances of disagreement between these reference groups on particular functions of presidents. These problems give rise to a number of questions. Is there a lack of consensus among the groups on the definition and functions of presidents' roles? Is there

\footnotetext{
'See Seventh-day Adventist Yearbook 2006, 435-486. In these pages Seventh-day Adventist colleges and universities are listed with various titles of chief executive officers.
} 
a difference between what the SDA senior college and university presidents actually do and what they are expected to do in their roles as perceived by the status groups?

\section{Purpose of the Study}

The purpose of this study was threefold: Purpose 1 was to examine the actual role performances of presidents of Seventh-day Adventist senior colleges and universities around the world that offered graduate degree programs in the 2004-2005 academic year, as perceived by five status groups, such as board chairs, presidents, academic vice presidents, student service vice presidents, and finance vice presidents. Purpose 2 was to examine the expected role responsibilities of presidents of Seventh-day Adventist senior colleges and universities around the world offering graduate degree programs, as expected by the same groups. Purpose 3 was to investigate the differences in perceptions between groups on actual role performances and expected roles of Seventh-day Adventist senior college and university presidents, as viewed by the same five status groups.

\section{Brief Review of Literature}

The literature review shows that the role of the president has changed since the start of the first colleges and universities in the selected world geographic regions included in this study. ${ }^{2}$ In 2003, Amaral, Meek, and Larsen edited a book that discussed the emergence of higher education's managerialism in eleven countries. ${ }^{3}$ Altbach and

${ }^{2}$ Judith A. Rile, "The Changing Role of the President in Higher Education," www.newfoundations.com/OrgTheory/Rile721.html (accessed January 14, 2008).

${ }^{3}$ Alberto Amaral, Vincent Lynn Meek, and Ingvild Marheim Larsen, eds., The Higher Education Managerial Revolution? Higher Education Dynamics Series. Vol. 3 (New York, NY: Springer, 2003), www.springer.com/education/journal/11162 (accessed March 27, 2008). 
Umazkoshi edited a volume in 2004 in which scholars wrote about several Asian universities and their presidencies in a number of countries. ${ }^{4}$ The articles in these two volumes present an account of evolving governance of colleges and universities. Cohen mentions five eras that reflect the evolution of higher education in America, characterized by new educational concerns and leadership roles. ${ }^{5}$

In the Colonial Era the president, a clergyman ${ }^{6}$ taught classes, administered discipline, preached at chapel, preached on Sunday, kept student records, kept the library, raised funds, and handled the day-to-day business of the college. His titles were master, president, rector, provost, principal, ${ }^{7}$ or chancellor. ${ }^{8}$

The Emergent Nation Era brought new challenges to the leadership roles. ${ }^{9}$

During this period presidents' responsibilities encompassed the full range of administrative duties later assigned to such subordinate functionaries as deans, registrar, bursar, treasurer, and so on. ${ }^{10}$

During the next 74 years that mark the University Transformation Era,

${ }^{4}$ Philip G. Altabach and Toru Umazkoshi, eds., Asian Universities: Historical Perspectives and Contemporary Challenges (Baltimore, MD: Johns Hopkins University Press, 2004).

${ }^{5}$ Arthur M. Cohen, The Shaping of American Higher Education: Emergence and Growth of the Contemporary System (San Francisco, CA: Jossey-Bass, 1998), 5.

${ }^{6}$ Cohen, 5 .

${ }^{7}$ Paul Westmeyer, An Analytical History of American Higher Education, $2^{\text {nd }}$ ed. (Springfield, IL: Charles C. Thomas, 1997), 19.

${ }^{8}$ Christopher J. Lucas, American Higher Education: A History, $2^{\text {nd }}$ ed. (New York, NY: Palgrave Macmillan, 2006), 124.

${ }^{9}$ Cohen, 56-83.

${ }^{10}$ Lucas, $120-124$. 
presidents' roles varied among institutions. Some presidents performed their roles nearly autocratically. Others developed hierarchical management system. ${ }^{11}$

The next three decades of Mass Higher Education Era, ${ }^{12}$ found presidents facing a complexity in management. Most institutions divided their administrative structure into three general functions: academic affairs, student affairs, and business affairs. Much of the president's responsibilities were delegated to his subordinate administrators. ${ }^{13}$

During the last 25 years of the twentieth century, considered as the Modern Era, the presidents had to deal with decreasing tuition revenue, increasing costs, demographic changes in the typical college-age population, and smaller pools of traditional students. The role of president has turned to a primarily administrative role. ${ }^{14}$

The twenty-first century has brought major changes to society. According to Rile, it is an age of super information technology. Today's college campuses are characterized by senior adults in college, replacing traditional teenage students; web-based courses at satellite locations; video conferencing; growing financial concerns; diverse groups many of which are unprepared for the discipline of learning because of poor study skills, lack of support, and language deficiencies; and myriad other demographic factors and contemporary issues. All of this seems to demand a changing role of the president. ${ }^{15}$

\footnotetext{
${ }^{11}$ Cohen, 152.

${ }^{12}$ Ibid., 5 .

${ }^{13}$ Ibid., 245.

${ }^{14}$ Ibid., 291-436.

${ }^{15}$ Rile, 6 .
} 
Role perceptions and role expectations have been theorized by various scholars and researchers who have served as college and university presidents. Lenski refers to role as a position which an individual occupies and to which is attached distinctive behavioral expectations and requirements. ${ }^{16}$ Owens stated that role expectation is the thinking that one individual has of the role behavior of another position occupier. ${ }^{17}$ According to Stogdill, Scott, and Jaynes, one who occupies the position has a different self-expectation than the others. ${ }^{18}$ The college/university president has many roles and functions. His or her self-expectation of roles could be different from expectations by others. According to Parsons, role conflict occurs because of such conflicting sets of agreed role expectations. ${ }^{19}$

Studies conducted during the past four decades on college presidents' roles reveal that there were agreement and disagreement between reference groups regarding their expectations for college/university presidents' roles and functions in certain areas.

Weeks's perception regarding amount of time spent by presidents on various roles was little different from that of Corson on the same variables. ${ }^{20}$ Two years later, Nelson, in

${ }^{16}$ Gerhard Lenski, Human Societies: A Macrolevel Introduction to Sociology (New York, NY: McGraw-Hill, 1970), 39.

${ }^{17}$ Robert G. Owens, Organizational Behavior in School (Englewood Cliffs, NJ: Prentice-Hall, 1970), 72 .

${ }^{18}$ Ralph M. Stogdill, Ellis L. Scott, and William E. Jaynes, Leadership and Role Expectations (Columbus, OH: Bureau of Business Research, College of Commerce and Administration, Ohio State University, 1956), 3.

${ }^{19}$ Talcott Parsons, The Social System (Glencoe, IL: Free Press, 1951), 25.

${ }^{20}$ John J. Corson, Governance of College and Universities (New York, NY: McGraw-Hill, 1960), 59-60, quoted in Ila D. Weeks, "The University President and the Public," Presidential Address, National Association of State Universities Transactions and Proceedings, 1950, 12-25. 
his study, found out that the status groups comprised of presidents and board members held diverging and converging expectations for college/university presidents' roles in certain areas. ${ }^{21}$ Hutchins's study detected conflicts and agreement between board members and presidents in certain role expectations. ${ }^{22}$ Oliver's study shows areas of congruence and non-congruence among most of the groups regarding perceptions and expectations on White presidents' roles. ${ }^{23}$ In his research Silas found areas of disagreement between status groups regarding role expectations of Black presidents. ${ }^{24}$ Kim's study, likewise, reveals potential conflicts between presidents, trustees, and faculty members regarding their expectations of certain areas of presidents' roles. ${ }^{25}$

I reviewed 27 faculty handbooks received from one ivy league university, two Catholic universities, two Protestant campuses, eight North American campuses, and 14 overseas campuses from 13 countries. The job descriptions found in these handbooks concur that the president is the chief executive who oversees all college or university operations through his or her vice presidents and other administrators.

${ }^{21}$ Lawrence O. Nelson, "Role Expectations for Selected College and University Presidents" (Ph.D. dissertation, Michigan State University, 1960), 136.

${ }^{22}$ Henry Thomas Hutchins, Jr., "Role Expectations of Selected College and University Presidents" (Ph.D. dissertation, University of Oklahoma, 1963), 158, quoted in Chong Wha Kim, "A Study of Role Expectations of Presidents in Seventh-day Adventist Colleges" (Ph.D. dissertation, University of Nebraska, 1975), 35.

${ }^{23}$ Arnold Robert Oliver, "The University President as Perceived by Boards of Trustees, Presidents, and Academic Vice Presidents in Selected Colleges and Universities" (Ph.D. dissertation, Southern Illinois University, 1972), 93-100.

${ }^{24}$ Samuel L. Silas, "Role Expectations of Presidents in Predominantly Black State and Privately Supported Colleges and Universities as Perceived by Trustees, Presidents, and Deans" (Ph.D. dissertation, Southern Illinois University, 1974), 143-157.

${ }^{25}$ Chong Wha Kim, "A Study of Role Expectations in Seventh-day Adventist College" (Ph.D. dissertation, University of Nebraska, 1975), 63-67. 


\section{Research Methodology}

This study was a quantitative, exploratory survey that was carried out through descriptive methods using a questionnaire with 47 questions and seven open-ended questions. The purpose of these questions was to elicit answers that showed the levels of actual role performances and expected role responsibilities of presidents and the differences that existed between these roles.

The population requested to participate in this study was limited to $(N=140)$ Seventh-day Adventist participants from 28 Seventh-day Adventist senior colleges and universities that offered graduate degree programs in the 2004-2005 school year in 17 countries, including the U.S.A., located in the 11 geographic and administrative world divisions of the General Conference of Seventh-day Adventist world church. The targeted population for the study was 140 administrators comprising 28 board chairs, 28 presidents, 28 academic vice presidents, 28 student service vice presidents, and 28 finance vice presidents of Seventh-day Adventist senior colleges and universities around the world that offered graduate degree programs on their campuses by their own authorities.

The sample consisted of 78 administrators, which included 18 board chairs, 17 presidents, 16 academic vice presidents, 13 student service vice presidents, and 14 finance vice presidents.

I modified the Oliver and Silas instruments and developed a survey instrument based on the Seventh-day Adventist educational philosophy, institutions' manuals, SDA literature, and a list of characteristics for effective college administration as identified by 
the Education Department of the Seventh-day Adventist world church. My instrument had seven broad categories of presidents' functions. Those were: student-related functions ( 8 items), employee-related functions ( 8 items), general administrative functions ( 9 items), finance-related functions ( 5 items), physical facilities-related functions ( 6 items), community- and alumni-related functions (6 items), and spiritual liferelated functions ( 5 items), making a total of 47 questions.

The instrument was sent to 15 Adventist educators and leaders for their responses, evaluations, and recommendations in regard to relevance and appropriateness of the contents, wordings of the questions, and the ease of completing the questionnaire. These educators, who had served as board chairs, presidents, academic vice presidents, deans of students, and chief financial officers in various colleges and universities in America and overseas, representing the five status groups of participants included in this study, were fully competent to validate the instrument. However, they were not among the target population that participated in the study. Upon the return of their comments and recommendations, a revised survey was formulated and submitted to my dissertation committee for its final approval.

Next, I sent the revised survey questionnaire to the target population $(N=140)$ in the month of September of 2004 with a personal cover letter and an endorsement letter from the education director of the Seventh-day Adventist world church, headquartered in Maryland, U.S.A. Since this was an international study, involving 16 countries outside of the U.S.A., the postal handling and completion of the questionnaires took an unusually long time. Two to five reminders were given to ensure an optimal response rate. The last 
completed questionnaire that was returned to me in the mail was May of 2006. The overall response rate was 58.6 percent $(n=82)$. Four cases were not included in the study because they did not meet the inclusion criteria.

The participants responded to 47 questions on two grids on a Likert-type continuum scale of 1 through 5 , with 1 being strong disagreement and 5 being strong agreement. One grid was for actual role performances and the other grid for expected role responsibilities. The data were run on SPSS statistical software to address the five research questions.

\section{Findings and Discussion}

Five research questions guided this study and were tested by 47 null subhypotheses for actual roles and 47 null sub-hypotheses for expected roles. The results of the statistical tests were organized and discussed in this section under these five research questions which were presented in chapter 1 . Research Questions 1 and 2 did not have any null hypotheses. Research Questions 3, 4, and 5 had their corresponding null hypotheses, 1,2, and 3, respectively, as stated in chapter 4 .

\section{Research Question 1}

Research Question 1 asked: What are the actual role performances of presidents of Seventh-day Adventist senior colleges and universities around the world, as perceived by their board chairs, presidents, academic vice presidents, student service vice presidents, and finance vice presidents?

This question was answered by descriptive statistics using mean scores for each of 
the responses to 47 questions on actual role performances. The groups perceived the presidents' actual role performances in four levels of performances, such as very strong role performance, strong role performance, moderate role performance, and weak role performance. See Tables 25 and 26 in chapter 4 and also Table 54 in Appendix J and Table 55 in Appendix K.

\section{Research Question 2}

Research Question 2 asked: What are the expected role responsibilities of presidents of Seventh-day Adventist senior colleges and universities around the world, as viewed by their board chairs, presidents, academic vice presidents, student service vice presidents, and finance vice presidents?

This question was answered by descriptive statistics using mean scores for each of the responses to 47 questions on expected role responsibilities of the presidents. The groups' responses were classified into four levels of expected role responsibilities, such as very high role expectation, high role expectation, moderate role expectation, and low role expectation. See Tables 27 and 28 in chapter 4 and also Table 54 in Appendix J and Table 55 in Appendix K.

\section{Findings and Discussion of Descriptive Statisties in Relation to Research Questions 1 and 2}

The findings and discussion of actual and expected roles in relation to Research Questions 1 and 2, respectively, are presented in four levels of perceptions of actual role performances and perceptions of role expectations of the respondents. 
Level 1: Very Strong Role Performance / Very High Role Expectation

Level 2: $\quad$ Strong Role Performance / High Role Expectation

Level 3: $\quad$ Moderate Role Performance / Moderate Role Expectation

Level 4: Weak Role Performance / Low Role Expectation

These results emanating from Tables 25 and 27 in chapter 4 are summarized in

Table 32. Since there is a high correlation between the actual and expected levels (Spearman rho $=.762, p=.000$ ), there is strong justification for the configuration of the four levels thus ignoring any other hybrid configurations. The discussion will therefore focus on Table 32 using the seven categories as the basis for organizing the findings.

\section{Student-Related Functions}

The majority of these items were of the moderate and weak levels on the expected side. These items were also of the moderate and weak levels on the actual side. The two items, 1 and 2, that were of a strong level on the expected side turned out to be more of a moderate level on the actual performance. Item 6 was expected a moderate role performance, but it was actually performed at a weak level. These three items had to do with student recruiting, working with student leaders, and teaching classes.

The other five items, $3,4,5,7$, and 8 of the student-related functions seem to be of little or no consequence for presidents' direct involvement and the above discrepancy suggests room for improvement on the part of the presidents in at least three areas.

Student recruitment, keeping in touch with student leaders, and teaching classes were found to be the specific areas that needed some improvement on the presidential roles. 
Table 32. A comparison of actual and expected role perceptions by level

\begin{tabular}{|c|c|c|c|c|}
\hline $\begin{array}{c}\text { Scale } \\
\#\end{array}$ & $\begin{array}{c}\text { Item } \\
\#\end{array}$ & Item & $\begin{array}{c}\text { Actual } \\
\text { Level }\end{array}$ & $\begin{array}{c}\text { Expected } \\
\text { Level }\end{array}$ \\
\hline & & Student-Related Functions & & \\
\hline \multirow{9}{*}{ I } & 1 & Participate in student recruitment & 3 & 2 \\
\hline & 2 & Work closely with student government leaders & 3 & 2 \\
\hline & 3 & Dialogue frequently with students & 2 & 2 \\
\hline & 4 & Visit students periodically in dorms during dorm worships & 3 & 3 \\
\hline & 5 & Take ultimate responsibility for student discipline & 3 & 3 \\
\hline & 6 & Teach some classes to maintain contact with students & 4 & 3 \\
\hline & 7 & Know every student personally & 4 & 4 \\
\hline & 8 & Serve as mentor of students & 3 & 3 \\
\hline & & Employee-Related Functions & & \\
\hline \multirow{9}{*}{ II } & 9 & Participate in faculty recruitment & 2 & 2 \\
\hline & 10 & Serve as academic leader of faculty & 3 & 3 \\
\hline & 11 & Guard faculty's academic freedom & 2 & 2 \\
\hline & 12 & Be responsible for submitting proposed academic changes to faculty & 3 & 3 \\
\hline & 13 & Work with faculty in reviewing accrediting agency's reports & 2 & 2 \\
\hline & 14 & Recommend for hiring, promotion, demotion, \&dismissal of faculty & 2 & 2 \\
\hline & 15 & Collaborate for advanced in-service training for faculty & 3 & 2 \\
\hline & 16 & Carry out policies and regulations pertaining to faculty & 2 & 2 \\
\hline & & General Administrative Functions & & \\
\hline \multirow{9}{*}{ III } & 17 & Prepare agenda for board of trustees' meetings & 1 & 1 \\
\hline & 18 & Preside over general faculty meetings & 2 & 2 \\
\hline & 19 & Take responsibility in structuring student admission standards & 3 & 3 \\
\hline & 20 & Take responsibility in strategic planning for institution & 2 & 1 \\
\hline & 21 & Take a leading role to prepare, revise $\&$ follow organizational chart & 2 & 1 \\
\hline & 22 & Foster research and intellectual climate for institution & 2 & 2 \\
\hline & 23 & Comply with legal mandates in addressing employee grievances & 2 & 1 \\
\hline & 24 & Help formulate and adhere to institution's educational philosophy & 2 & 1 \\
\hline & 25 & Delegate responsibility, authority, and resources to administrators & 2 & 1 \\
\hline
\end{tabular}


Table 32-Continued.

\begin{tabular}{|c|c|c|c|c|}
\hline $\begin{array}{c}\text { Scale } \\
\#\end{array}$ & $\begin{array}{l}\text { Item } \\
\#\end{array}$ & Item & $\begin{array}{l}\text { Actual } \\
\text { Level }\end{array}$ & $\begin{array}{l}\text { Expected } \\
\text { Level }\end{array}$ \\
\hline & & Finance-Related Functions & & \\
\hline \multirow{6}{*}{ IV } & 26 & Participate in the preparation of annual budget of the institution & 2 & 2 \\
\hline & 27 & Ensure that institution's budget, after approval by board, is adhered to & 2 & 2 \\
\hline & 28 & Present institution's annual audited financial statements to board & 3 & 3 \\
\hline & 29 & Recommend to board the salary changes for positions & 3 & 2 \\
\hline & 30 & Accept accountability to board for financial operation of institution & 2 & 1 \\
\hline & & Physical Facilities-Related Functions & & \\
\hline \multirow{7}{*}{ V } & 31 & Present to board the physical needs of institution & 2 & 2 \\
\hline & 32 & Direct the preparation of plans for new buildings and installations & 3 & 2 \\
\hline & 33 & Plan for major repairs and alterations of plant, buildings \& equipment & 3 & 3 \\
\hline & 34 & Recommend to board for equipment acquisitions & 3 & 3 \\
\hline & 35 & Ensure that physical plant master plans are current $\&$ followed closely & 2 & 2 \\
\hline & 36 & Ascertain that grounds are well kept through appropriate officers & 2 & 2 \\
\hline & & Community- and Alumni-Related Functions & & \\
\hline \multirow{7}{*}{ VI } & 37 & Assist in raising funds for support of institution & 2 & 1 \\
\hline & 38 & Represent institution to churches of the constituency & 2 & 1 \\
\hline & 39 & Present reports about college/university to constituency and alumni & 2 & 1 \\
\hline & 40 & Represent institution as its official spokesperson to public & 2 & 1 \\
\hline & 41 & Associate closely with alumni groups & 2 & 1 \\
\hline & 42 & Listen to constituency & 2 & 1 \\
\hline & & Spiritual Life-Related Functions & & \\
\hline \multirow{5}{*}{ VII } & 43 & $\begin{array}{l}\text { Be responsible for promotion of religious life of students in the } \\
\text { institution via delegation }\end{array}$ & 2 & 2 \\
\hline & 44 & $\begin{array}{l}\text { Be responsible for promotion of religious life of faculty and staff in the } \\
\text { institution via delegation }\end{array}$ & 2 & 2 \\
\hline & 45 & $\begin{array}{l}\text { Be responsible for chapels, vespers, weeks of spiritual emphasis and } \\
\text { other religious programs }\end{array}$ & 3 & 2 \\
\hline & 46 & Manifest Christ-like character in spiritual leadership & 2 & 1 \\
\hline & 47 & Focus on Christian philosophy of the institution & 1 & 1 \\
\hline
\end{tabular}

Note: 1 = Very Strong Actual Role/Very High Expected Role; 2 = Strong Actual Role/High Expected Role; 3 = Moderate Actual Role/Moderate Expected Role; and 4 = Weak Actual Role/Low Expected Role. 
Student recruitment was expected to be a strong role of the president. McAllisterWilson, Williford, and Neelands maintained that presidents should be involved in student recruitment to ensure a certain level of quality enrollment to fulfill the college's mission. ${ }^{26}$

Students are the college/university's mission, or at least are the primary means by which an institution accomplishes its mission. Students are the organic link between the president and the faculty. A president may not have enough time to transform the quality and character of a faculty, but he or she must have time to affect the nature of the student body. ${ }^{27}$ In a document titled "Characteristics of a Successful Seventh-day Adventist College or University," the Education Department of the Seventh-day Adventist world church, headquartered in Maryland, U.S.A., has emphasized the student-related functions of administration..$^{28}$

\section{Employee-Related Functions}

The majority of these items were of the strong level on the expected side. These items were also of the strong level on the actual performance side. The two items, 10 and

${ }^{26}$ David McAllister-Wilson, Craig Williford, and David Neelands, "The President's Role in Enrollment Management and Student Issues," in A Handbook for Seminary Presidents, ed. G. Douglass Lewis and Lovett H. Weems, Jr. (Grand Rapids, MI: William B. Eerdmans, 2006), 143. The authors maintained that the president's role in student recruitment and enrollment should be guided by a marketing plan, budget and technological resources, and academic offerings.

${ }^{27}$ McAllister-Wilson, Williford, and Neelands, in $A$ Handbook for Seminary Presidents, ed. Lewis and Weems, Jr., 142.

${ }^{28}$ The Education Department directors attending the World Education Advisory at the General Conference Headquarters, April 1996, issued a document containing twelve characteristics of a successful Seventh-day Adventist college or university, which was revised March 1997. Section 6 of this document contains a paragraph dealing with students. See Appendix D. 
12 , that they were expected to perform moderately on, they actually performed moderately on them. These items had to do with faculty academic matters, which is not surprising, because the academic vice presidents are charged with these responsibilities. However, there was one academic matter that was deemed important and the presidents all recognized this to be the case and acted accordingly. This had to do with guarding faculty's academic freedom. Seventh-day Adventists acknowledge academic freedom to pursue knowledge and truth that are not opposed to their religious faith. ${ }^{29}$

All the other five items, $9,11,13,14$, and 16 , were of the strong levels for both expected and actual sides and this shows that the presidents were meeting expectations as far as employee-related roles were concerned. This bodes well for stimulating a healthy climate on the campuses that could translate into high morale among the employees.

The findings suggest that while believing that "faculty members are the body and the heart of a college or university, ${ }^{, 30}$ the presidents were engaged in their roles of hiring, promotion, demotion, dismissal, and retention of employees, keeping in mind that there is "a major shortage of teachers. ${ }^{31}$ Many of the institutions are multi-cultural in terms of students and faculty and staff. Hence, employee management should be done with great care. ${ }^{32}$ Seventh-day Adventist college and university presidents are expected to commit

\footnotetext{
${ }^{29}$ See the General Conference Executive Committee's 1987 Annual Council General Actions, pp. 17-19, quoted in Adventist Review 166, no. 9 (1989): 22.

${ }^{30}$ James L. Fisher and James V. Koch, Presidential Leadership: Making a Difference (Phoenix, AZ: American Council on Education and Oryx Press, 1996), 146.

${ }^{31}$ Gene A. Budig, “A Perfect Storm," Phi Delta Kappan 88, no. 2 (2006): 114.

${ }^{32}$ Daryl G. Smith, Caroline S. Turner, Nina Osei-Kofi, and Sandra Richards, "Interrupting the Usual: Successful Strategies for Hiring Faculty," The Journal of Higher Education 75, no. 2 (2004): 133156.
} 
themselves to looking after the professional interests of the faculty and staff. ${ }^{33}$

\section{General Administrative Functions}

Most of these items were of the very strong level on the expected side. Except one item, all of the items were of the strong level on the actual performance side. Thus, the results yielded an encouraging picture of presidents' performances.

However, the findings indicate that although the presidents were meeting expectations in four of the nine areas, they needed to improve on the remaining five areas, such as strategic planning; following organizational chart; addressing employee grievances; adhering to educational philosophy; and delegating responsibilities. Cooley, Klein, and Weeks suggested that presidents give a greater attention to these roles. ${ }^{34}$

One item was on both very strong actual performance side and very high expectation side. This had to do with preparing agenda for board of trustees' meetings. This shows that presidents were doing excellent in working with the governing boards of their institutions. They were expected to keep up this trend.

Only one item was performed moderately and was expected to perform moderately as well. This had to do with structuring student admission standards, which is not surprising. The admissions office under the guidance of academic deans and enrollment vice president or enrollment director takes charge in student admissions, relieving the president of his or her direct involvement in this matter, although the

\footnotetext{
${ }^{33}$ See Appendix D, sec. 4.

${ }^{34}$ Robert Cooley, Christa Klein, and Louis Weeks, "The President's Role in Governance," in $A$ Handbook for Seminary Presidents, ed. Lewis and Weems, Jr., 36-56.
} 
president is ultimately held responsible in this area also, being the chief executive officer of the institution. The president is expected to take interest in student admissions to ensure a quality enrollment for the sake of marketability and financial aid. ${ }^{35}$ In short, the Seventh-day Adventist senior college and university presidents are expected to have a high level of role performances in all areas of general administration. ${ }^{36}$

\section{Finance-Related Functions}

The majority of these items were of the strong level on the expected side. One item was on the very high expectation level. The findings suggest that although the presidents demonstrated a strong actual role performance level in the majority of the finance-related functions, they were expected to do even better, especially, in the area of financial accountability. Ruger, Canary, and Land clearly stated that presidents should give priority attention to the college/university's financial condition and performance, including budget development and budget review. While there may be a vice president for finance or a chief financial officer charged with specialized duties in these areas, the policies, practices, and results ultimately are the president's responsibility to ensure that the institution's mission is the center of financial activities. The chief executive officers of for-profit organizations immersed in financial scandals may try to deflect responsibility onto their financial officers, but by and large the CEOs are held accountable for the activities on their watch. The same holds true for Christian schools' chief executive

\footnotetext{
${ }^{35}$ See Appendix D, sec. 8.

${ }^{36}$ See Appendix D, sec. $2 \& 3$.
} 
officers, ${ }^{37}$ more precisely, the SDA college/university presidents.

Two items, 28 and 29, were of the moderate level on the actual side. One of them had to do with presentation to the board regarding the institution's annual audited financial statement which the presidents were expected to perform moderately. This is not surprising because the finance vice president or chief financial officer is generally delegated with this responsibility, with the understanding that he or she updates the president on the matter.

The other actual moderate performance item was expected to be performed on a high performance level. This had to do with recommending to the board the salary changes for the faculty and staff. Although finance officers are vested with financial responsibilities, the presidents may, at times, deem it necessary to wear the hat of "business manager" as well, and assume the ultimate responsibility in overseeing the financial planning and financial management, including employee compensations. ${ }^{38}$ Jean Dowdall, a distinguished educator, social scientist, and administrator who served as first woman president of Simmons College, mentioned that the college/university presidents must have the financial skill and accountability to help operate their institutions well. ${ }^{39}$ The governing boards of the Seventh-day Adventist colleges and universities expect their

\footnotetext{
${ }^{37}$ Anthony Ruger, John Canary, and Steven Land, "The President's Role in Financial Management," in $A$ Handbook for Seminary Presidents, ed. Lewis and Weems, Jr., 92-100; see also Thomas Edward O'Connell, "The Five Roles of the College or University President"' (Ed.D. dissertation, University of Massachusetts, 1974), 65.

${ }^{38}$ Ruger, Canary, and Land, in Lewis and Weems, Jr., 92.

${ }^{39}$ Jean A. Dowdall, Searching for Higher Education Leadership: Advice for Candidates and Search Committees (Westport, CT: Praeger Publishers, 2007), 126.
} 
presidents to pursue a careful stewardship of financial resources. ${ }^{40}$

\section{Physical Facilities-Related Functions}

The majority of the items were found to be of strong level on the expected side. Except one item, these items were also of the strong level on the actual performance side.

The two items, 33 and 34 , that presidents were expected to perform moderately on, they actually performed moderately on them. These items had to do with the planning for major repairs of equipment and alterations of plant; and recommending to board for equipment acquisitions. This is not surprising because the vice presidents for finance are usually delegated with these responsibilities and they work through the directors of plant and custodial services, relieving the presidents of their direct involvement in these functions.

All items of physical facilities-related functions were found to be meeting the expectations, whether high or moderate, except one item. This had to do with the presidents' further involvement in directing the preparation of plans for new buildings and installations on campus. This is understood because it is the chief executive who is ultimately responsible for adhering to the physical plant master plan. Plans for new buildings and major installations on the grounds are part of this master planning.

The findings suggest that every educational institution has an assigned unit administrator who heads a team of specialized people entrusted with the responsibility of proper "preventive maintenance" of physical plants, buildings, equipment, and other

\footnotetext{
${ }^{40}$ See Appendix D, sec. 8 .
} 
campus physical facilities." ${ }^{\text {"4l }}$ However, the president, as stated by Arthur Hansen, works closely with his or her support administrators in these areas. ${ }^{42}$ The president's primary duty cannot be focused on facilities, as there are other issues of leadership, program, personnel, and development that demand his or her greater attention.

However, inadequate or poorly functioning facilities have a major negative impact on the quality of life in the college or university. Ewing, Graves, and Landrebe suggested that the president, working with the board, must provide strategic leadership and set priorities to ensure appropriate maintenance and improvements to facilities, keeping the focus on the mission and vision of the institution. He or she does not require special expertise or an inordinate amount of time, but must insist on appropriate planning and budgeting for facility. ${ }^{43}$ In short, Seventh-day Adventist college and university presidents are expected to be aware of the needs of physical facilities and fulfill them. ${ }^{44}$

\section{Community- and Alumni-Related Functions}

All six items were of the very strong level on the expected side. These same items were of the strong level on the actual performances side. The findings reveal that SDA presidents worldwide were found doing better in these areas. This is the only

\footnotetext{
${ }^{41}$ Phil Waier, Steve Plotner, and Andrea Keenan, Preventive Maintenance for Higher Education Facilities: A Planning and Budgetary Tool for Facilities Professionals (Kingston, MA: R.S. Means, 2002), pp. ix-xii.

${ }^{42}$ Arthur G. Hansen, "President and Physical Plant Administrator Face Common Goal of Providing an Efficient Educational Environment," Association of Physical Plant Administrators of Universities and Colleges 5, no. 1 (1975): 1-7.

${ }^{43}$ Ward Ewing, Thomas Graves, and Robert Landrebe, "The President's Role in Managing Facilities," in A Handbook for Seminary Presidents, ed. Lewis and Weems, Jr., 106-124.

${ }^{44}$ See Appendix D, sec. 9.
} 
category of functions that showed strong and very strong levels for all items on the actual and expected sides, respectively. It means that while presidents actually performed these roles strongly, there was still room for more improvement in the areas of fundraising and public relations activities.

The presidents are expected to improve on each of the community- and alumnirelated responsibilities. Studies indicate that fundraising and friend-raising efforts are perhaps more vital today than ever before. The ever-rising cost of education, the everexisting shortage of funds, the pressing complexity of the multicultural society challenged by myriad issues and trends, and the new technological innovations, all seem to demand a productive marketability of college programs. This marketability is possible through effective public relations and alumni activities that are so essential for survival of a college or university in the twenty-first century.

The findings suggest that the presidents in all the SDA colleges and universities worldwide made efforts to reach out to the public, community, churches, and alumni through visits, speeches, and various modes of communication as suggested by the Education Department of the SDA world church. ${ }^{45}$ I can give one example of Andrews University, the premiere institution of the Seventh-day Adventist world church, regarding its recent public and alumni relations activities and their outcomes. Andrews University has been introducing innovative ideas, attractive strategies, and trustworthy fundraising programs among its worldwide alumni and friends, and local community and churches. This has resulted in a notable increase over the past five years in student enrollment,

\footnotetext{
${ }^{45}$ See Appendix D, sec. 10.
} 
tuition revenue, ${ }^{46}$ annual giving, and contributions from alumni and donors. ${ }^{47}$ Public relations do play a vital role in the survival of colleges and universities. ${ }^{48}$

\section{Spiritual Life-Related Functions}

Three items, 43, 44, and 45, were of the strong level and two items, 46 and 47, were of the very strong level on the expected side. The majority of these items were of the strong level on the actual role performance side. The two items that were performed strongly and were also expected to perform strongly had to do with the responsibilities of promoting religious life of students and faculty in the institution. This shows that presidents' actual performances were meeting the expectations in these two areas.

The item that had to do with manifesting a Christ-like character in spiritual leadership roles was of the strong actual role performance level and was expected to be carried out on the highest level of performance.

Another item was performed moderately, but it was expected to be performed with a strong emphasis. This item had to do with taking responsibility for chapels, vespers, weeks of spiritual emphasis, and other religious programs on the college and university campuses. The findings suggest that the presidents, by and large, have given a moderate attention to the religious functions. The reason could be that the college and

\footnotetext{
${ }^{46}$ Niels-Erik Andreasen, "President's Annual Report-2008," Focus: The Andrews University Magazine 44, no. 4 (2008): 48. President Andreasen of Andrews University gave a comparative report of student enrollment and tuition increase covering the period from 2004-2008.

${ }^{47}$ Ibid., 51, 68.

${ }^{48}$ Garrett W. Nudd, "Practical Relations: Making Your School Stand Out Despite Limited Financial Resources," The Journal of Adventist Education 64, no. 1 (2001): 9-13.
} 
university chaplains had carried out these responsibilities, relieving the presidents of their direct involvement in these areas.

The results of this study also suggest that the presidents were expected to do more than their actual roles which they performed moderately. They were to be more involved in the conduct of chapels, vespers, weeks of spiritual emphasis, and other religious programs. These religious components make the Seventh-day Adventist colleges and universities unique in their mission and philosophy from the other non-SDA institutions. The Education Department of the Seventh-day Adventist world church has promoted the importance of implementing campus-wide spiritual programs for all. ${ }^{49}$

According to Lawrence Geraty, Seventh-day Adventist colleges and universities are similar to others in many ways, but an Adventist institution is Adventist because of its essential characteristics of identity that give its singular mission. ${ }^{50}$ Norman Maphosa indicated that administrative skills needed for Seventh-day Adventist college and university presidents are no different from those of their counterparts in other similar institutions. The differences are found in the underlying worldview brought to the workplace by the chief executives. ${ }^{51}$ Such distinctive identity, unique mission, and underlying worldview of the SDA higher education demonstrate a special kind of presidential spiritual functions, both in actual practice and role expectations.

\footnotetext{
${ }^{49}$ See Appendix D, sec. 7.

${ }^{50}$ Lawrence T. Geraty, "The Essential Characteristics of SDA Higher Education," The Journal of Adventist Education 56, no. 4 (1994): 5.

${ }^{51}$ Norman Maphosa, "Critical Skills for Chief Executives in Adventist Institutions of Higher Learning," in Christ in the Classroom: Adventist Approaches to the Integration of Faith and Learning, ed. Humbarto M. Rasi (Silver Spring, MD: Institute for Christian Teaching, 1994), 12:144.
} 
One item that presidents were expected to perform very strongly on, they actually performed very strongly on it too. This had to do with focusing on Christian philosophy in all areas of presidents' functions. Seventh-day Adventists have a distinct philosophy that exists as a common thread in matters of administration of SDA colleges and universities worldwide..$^{52}$

Ellen White, one of the founders of the Seventh-day Adventist world church, stated that the mission or philosophy of Seventh-day Adventist education is to prepare "the student for the joy of service in this world and for the higher joy of wider service in the world to come." ${ }^{53}$ The unique Christian worldview, values, and philosophy, reflected in the mission statements, provide vision for students, faculty, and institutional excellence and serve as effective tools ${ }^{54}$ and the guiding principle ${ }^{55}$ for overall management of universities and colleges.

The presidents of SDA colleges and universities were all and will always be Seventh-day Adventists, who, in their sincere convictions, are genuinely wedded to the Seventh-day Adventist Christian philosophy of education, which is further explained in our time by such Adventist college and university presidents and scholars as John

\footnotetext{
${ }^{52}$ Sylvan Lashley, "Should Adventist Colleges and Universities Differ From One Another?" The Journal of Adventist Education 65, no. 2 (2002/2003): 7.

${ }^{53}$ White, Education, 13; see also Seventh-day Adventist Encyclopedia, 1996 ed., s.v. "Education, Seventh-day Adventist Philosophy Of." Ellen Gould White was one of the founders of the Seventh-day Adventist world church.

${ }^{54}$ Jack Meacham, "What's the Use of a Mission Statement?" ACADEME: Bulletin of the American Association of University Professors 94, no. 1 (2008): 21.

${ }^{55}$ John C. Scott, "The Mission of the University: Medieval to Postmodern Transformations," The Journal of Higher Education 77, no. 1 (2006): 1-33.
} 
Fowler, ${ }^{56}$ George Knight, ${ }^{57}$ Sylvan Lashley, and Lawrence Geraty. Considering all this, regardless of geographic locations, administrative structures, and size and type of institutions, the Seventh-day Adventist senior college and university presidents around the world demonstrated a very high level of Christian philosophy in all their actual role performances and expected role responsibilities.

In summary, the answers to Research Questions 1 and 2 were found in the identification of the prominent themes that were embedded in all of the outstanding actual and expected roles, among which mission and philosophy of institution were the topmost. The other themes centering around public relations, fundraising, strategic planning, legal mandates, organizational protocols, delegation of responsibilities, accountability, and, most importantly, servant leadership, were found to be the important areas of presidents' actual and expected roles in the Seventh-day Adventist colleges and universities. Most of the student-related functions examined were not considered important as far as the findings of this study are concerned.

\section{Research Question 3}

Research Question 3 asked: Is there a difference in perception between groups of board chairs, presidents, academic vice presidents, student service vice presidents, and finance vice presidents of Seventh-day Adventist senior colleges and universities around the world regarding their senior college/university presidents' perceived actual role

\footnotetext{
${ }^{56}$ John M. Fowler, "Philosophy and Christian Education: A Road to Despair or a Highway to Understanding?" The Journal of Adventist Education 69, no. 2 (2007): 4-9.

${ }^{57}$ George R. Knight, "Why Have Adventist Education?" The Journal of Adventist Education 67, no. 5 (2005): 6-9.
} 
performances? This research question was answered with Null Hypothesis 1 for all null sub-hypotheses, 1-47, as presented in chapter 4.

\section{Research Question 4}

Research Question 4 asked: Is there a difference in expectation between groups of board chairs, presidents, academic vice presidents, student service vice presidents, and finance vice presidents of Seventh-day Adventist senior colleges and universities around the world regarding their senior college/university presidents' expected role responsibilities? This research question was answered with Null Hypothesis 2 for all null sub-hypotheses, 1-47, as presented in chapter 4.

\section{Findings and Discussion of Null Hypotheses 1 and 2 in Relation to Research Questions 3 and 4, Respectively}

Null Hypothesis 1 stated that there is no difference among the status groups (board chairs, presidents, academic vice presidents, student service vice presidents, and finance vice presidents) on each of the perceived actual role performances of presidents of Seventh-day Adventist senior colleges and universities around the world.

Null Hypothesis 2 stated that there is no difference among the status groups (board chairs, presidents, academic vice presidents, student service vice presidents, and finance vice presidents) on each of the expected role responsibilities of presidents of Seventh-day Adventist senior colleges and universities around the world.

A summary of the one-way analyses of variance results and accompanying discussion is presented in light of the levels of agreement or disagreement for actual and 
expected roles among the five status groups of the seven major categories of presidents' responsibilities studied. The findings indicate that although differences were found among the status groups for perceived actual role performances and expected roles on the 47 role items, none of these differences was statistically significant. The status groups were therefore in consensus in every aspect of the operation of the Seventh-day Adventist senior colleges and universities around the world, both for the role expectations and actual role performances.

\section{Student-Related Functions}

Statistical data in chapter 4 revealed a high degree of consistency in agreement between the responding groups regarding the presidents' perceived actual role performances and expected roles in student-related functions. The results indicate that no matter how the perceptions and expectations of the groups, they were in consensus on all eight items, 1-8. They shared the same views on the presidents' actual and expected performances in student recruitment; working with student leaders; frequent dialoguing with students; dorm visitations; student discipline; class teaching; knowing students personally; and mentoring students. ${ }^{58}$ The findings suggest that there was a general consensus between the groups on the actual and expected student-related functions of the presidents of the Seventh-day Adventist senior colleges and universities around the world.

${ }^{58}$ The ANOVA results presented in Table 33 in Appendix $\mathrm{G}$ and Table 40 in Appendix $\mathrm{H}$ showed no significant statistical differences between the five respondent groups on the actual and expected studentrelated roles of the presidents. The board chairs, presidents, academic vice presidents, student service vice presidents, and finance vice presidents agreed among themselves on the degrees of the student-related roles. 
In contrast to my findings, Arnold Oliver, in his 1972 study, found disagreement among the presidents, board members, and academic deans on most of the student-related functions of the presidents in selected White colleges and universities belonging to the American Association of State Colleges and Universities. ${ }^{59}$

Two years later, in 1974, Samuel Silas conducted a study among the Black presidents of predominantly Black state supported and private senior colleges and universities. He found no major differences among the presidents, board members, and academic deans in most of the student-related functions of presidents. ${ }^{60}$

In 1975, Chong Kim found potential conflict between the presidents, board members, and faculty members of the Seventh-day Adventist colleges and universities in the United States in some student-related functions. ${ }^{61}$ More than three decades have elapsed since Oliver and Kim have found disagreement between the respondent groups in some areas of the student-related functions of the college and university presidents in the United States.

My study, on the other hand, did not find differences of perceptions of actual role performances and role expectations between the groups regarding any of the presidents' eight student-related functions examined. This cohesiveness among the five status groups is indeed remarkable. The factors that could help I believe bring this unity of action in the administration of the SDA colleges and universities globally are the institutions'

\footnotetext{
${ }^{59}$ Oliver, 96.

${ }^{60}$ Silas, $144,145$.

${ }^{61} \mathrm{Kim}$, 63-66.
} 
purposes, ${ }^{62}$ identity, ${ }^{63}$ unique Christian worldview, ${ }^{64}$ mission,,${ }^{65}$ philosophy, ${ }^{66}$ and essential characteristics. ${ }^{67}$ I tend to conclude that these factors have served and were expected to serve as the guiding principle in the operation of the SDA colleges and universities.

\section{Employee-Related Functions}

Data in chapter 4 showed consensus in agreement among the five responding groups regarding the presidents' perceived actual role performances and expected roles in employee-related responsibilities. The findings suggest that regardless of perceptions and expectations, the groups were in agreement on all eight functions in this category, Items 9-16.

The responding groups held the same views on the presidents' perceived actual role performances and expected role responsibilities in the areas, such as participating in the faculty recruitment; serving as the academic leader; guarding the academic freedom of the faculty; proposing changes in academic matters; working with the faculty in reviewing and implementing the accrediting agency's reports; recommending for hiring, promotion, demotion, and dismissal of faculty; arranging in-service training for the

${ }^{62}$ Walton J. Brown, A Handbook for Seventh-day Adventist School Administrators (Takoma Park, MD: Department of Education, General Conference of Seventh-day Adventists, 1980), 9. Brown served as an associate director of the Education Department at the General Conference of Seventh-day Adventist world church located in Maryland, U.S.A.

${ }^{63}$ Geraty, 5 .

${ }^{64}$ Maphosa, in Christ in the Classroom, (1994), 12:144.

${ }^{65}$ See n. 53.

${ }^{66}$ Lashley, Fowler, and Knight. See nn. 52, 56, 57.

${ }^{67}$ Brown, 31-34. 
employees; and carrying out the policies for the faculty and staff. ${ }^{68}$

More than three decades ago Oliver, ${ }^{69} \mathrm{Silas}^{70}$ and $\mathrm{Kim}^{71}$ surveyed selected presidents, board members, faculty members, and academic deans or academic vice presidents in the United States to determine their perceptions regarding the presidents' role expectations. Oliver's study showed differences between the board members, presidents, and academic vice presidents regarding their perceptions of faculty-related responsibilities of the presidents in selected White colleges and universities. The Silas study, in contrast, found no differences among the board members, presidents, and academic vice presidents in their views regarding faculty-related roles of the presidents in selected Black colleges and universities. Kim identified the conflict among the board members and faculty members on some of the faculty-related roles of the presidents in the Seventh-day Adventist colleges and universities in the United States. ${ }^{72}$

The present investigation, conducted more than three decades after the Oliver, Silas, and Kim studies were done, found that there was a high degree of consensus between the groups of respondents on each of the actual and expected roles in this category. This is a good picture. It shows that the SDA college and university presidents

${ }^{68}$ The ANOVA results presented in Table 34 in Appendix G and Table 41 in Appendix $\mathrm{H}$ showed no significant statistical differences between the five groups on the actual and expected roles, respectively, in the presidents' employee-related functions. The board chairs, presidents, academ ic vice presidents, student service vice presidents, and finance vice presidents agreed among themselves on the degrees of the presidents' actual and expected roles in the employee-related area.

${ }^{69}$ Silas, $4-8$.

${ }^{70}$ Oliver, 6-11.

${ }^{71}$ Kim, 4-5.

${ }^{72}$ Ibid., 64-65. 
worldwide did their employee-related jobs and were expected to do the same as outlined in the job descriptions without any disagreement in perceptions and expectations on the part of the five respondent groups. One of the main reasons for this cohesiveness could be attributed to the unique identifying features that Walton Brown ${ }^{73}$ mentioned could make the SDA colleges and universities different from all the other non-SDA institutions around the world and that which would guide the presidents in their functions.

\section{General Administrative Functions}

The respondent groups showed a high degree of consistency in agreement among themselves in their perceptions and expectations of all nine general administrative functions of presidents, Items 17-25, both for the actual and expected roles. They did not differ significantly among themselves on any of these role items. The findings suggest that they shared similar views regarding the presidents' perceived actual performances and expected functions in these areas. These particular functions are: preparing agendas for board meetings; presiding over general faculty meetings; taking responsibility in structuring student admission standards; taking responsibility in strategic planning; reviewing and implementing organizational chart; fostering research climate; addressing employee and student grievances; adhering to educational philosophy; and delegating responsibility, authority, and needed resources to appropriate administrators. ${ }^{74}$

\footnotetext{
${ }^{73}$ Brown, 31-34.
}

${ }^{74}$ The ANOVA results presented in Table 35 in Appendix $\mathrm{G}$ and Table 42 in Appendix $H$ showed no significant statistical differences between the five groups on the actual and expected roles, respectively, in the presidents' general administrative functions. The board chairs, presidents, academic vice presidents, student service vice presidents, and finance vice presidents agreed among themselves on the degrees of actual and expected roles of the presidents in general administration 
Oliver's study found differences in perceptions among the respondents regarding the presidents' role expectations in general administrative responsibilities. ${ }^{75}$ Silas's study showed differences among the same category of respondent groups in most of the administrative functions. ${ }^{76}$ Kim detected many conflict items of administrative functions between the board members and faculty members. ${ }^{77}$

The results of my study showed otherwise. The board chairs, college/university presidents, and their support administrators did not differ among themselves in their opinions as to how the presidents actually performed and how they were expected to perform in their general administrative responsibilities. This cohesiveness could be attributed to the respondents' awareness of these functions which were evidently put into practice and were expected to be performed by the presidents in the Seventh-day Adventist senior colleges and universities around the world. ${ }^{78}$ In this study no potential role conflicts in general administration occurred between any of the groups. The board chairs, presidents, and three groups of vice presidents worldwide are similar in that there is a common consensus among them regarding how they perceived the presidents performed and how they expect them to perform their administrative duties in the SDA colleges and universities. This cohesiveness uplifts the general tone of the campus.

$$
\begin{aligned}
& { }^{75} \text { Oliver, } 96 . \\
& { }^{76} \text { Silas, 144-145. } \\
& { }^{77} \mathrm{Kim}, 63-66 . \\
& { }^{78} \text { Colleges and universities have operating manuals in which job descriptions of presidents are }
\end{aligned}
$$
provided. Many colleges and universities have sent in their faculty handbooks to me for this research. Also, the General Conference Education Department of the Seventh-day Adventist world church has some guidelines for successful colleges and universities. See Appendix D. 
Another shift in the presidents' emphasis in terms of time spent on various aspects of functions has taken place during the past four decades. John Corson mentioned that 10 percent of the president's time was allocated to general administration. Later, Ila Weeks subscribed to this opinion. ${ }^{79}$ Results of my study indicate the groups' agreement regarding a higher percentage of time spent on the presidents' actual and expected roles of the general administrative functions. ${ }^{80}$ This is understood because with the passage of time, sophistication of technology, and demand by the stakeholders, the presidents are challenged with higher expectations on all fronts of their responsibilities.

\section{Finance-Related Functions}

There were five items in this category, Items 26-30. The results revealed that the respondents' perceptions did not differ significantly between them on any of these items, both actual and expected roles. The findings indicate that all five groups held similar views on how the presidents have actually performed and how they expected them to perform in the financial area. These financial functions are: participating in the preparation of the institution's annual operating budget; adhering to the operating budget after it is approved by the governing board; presenting annual audited financial statements to the board; recommending to the board the salary changes for faculty and staff; and accepting financial accountability to the board in the operation of the institution. ${ }^{81}$ There

${ }^{79}$ Corson, 12-25.

${ }^{80}$ Compare expected level with actual level in Table 32, sec. III.

${ }^{81}$ The ANOVA results presented in Table 36 in Appendix G and Table 43 in Appendix H showed no differences between the groups on the actual and expected roles, respectively, in presidents' financerelated functions. 
was a general consensus around the world between the respondent groups regarding the perceived actual performances and expected roles of the SDA senior college and university presidents on each of the financial items.

More than three decades ago some studies were done on the college and university presidents' role expectations. Oliver's study showed a strong consensus among the presidents and board members regarding their perceptions and expectations on a majority of the financial roles of the university presidency in selected colleges and universities belonging to the American Association of State Colleges and Universities. Although academic vice presidents tended to differ more often from the board members and presidents, they were in consensus among themselves. ${ }^{82}$ Silas found consensus between the trustees, presidents, and academic vice presidents in their perceptions of role expectations of Black presidents in financial matters in selected Black colleges and universities in the Eastern states. ${ }^{83}$ Kim's study identified only one conflict item of the financial functions that was observed existing between the presidents and board members of the Seventh-day Adventist colleges and universities in the United States. ${ }^{84}$

In my study I found the groups to be similar, not differing from one another in their perceptions and expectations of the presidents' finance-related functions. Literature attributed 40 percent of the president's time to financial matters. ${ }^{85}$ Examination of the

\footnotetext{
${ }^{82}$ Oliver, 93-95.

${ }^{83}$ Silas, 143-145.

${ }^{84} \mathrm{Kim}, 63$.

${ }^{85}$ Corson, 12-25.
} 
data in Tables 26 and 28 , in chapter 4 of this study, would indicate a lower percentage for the actual roles. The reason for this lower percentage of the president's time spent in financial functions could be that in the present age all respondent groups seem to hold a similar view that the financial matters are delegated to the chief financial officer of the institution. Additionally, this observation is indicative of a general consensus between the groups regarding the financial functions of the presidents of the SDA colleges and universities worldwide.

\section{Physical Facilities-Related Functions}

There were six variables, Items $31-36$, in this section. The results revealed a high degree of consistency in agreement between the groups surveyed regarding the presidents' actual and expected roles in this category of functions. The findings suggest that regardless of the perceptions and expectations of the respondent groups, there was a consensus observed among them on all six physical facilities-related functions of the presidents. These items had to do with presenting the physical needs of the institution to the board; directing the preparation of plans for new buildings and installations; planning for major repairs and alterations of plant, buildings, and equipment; recommending to the board for equipment acquisition; ensuring that the physical plant master plans are current and followed closely; and ascertaining that the campus grounds are well-kept through appropriate officers. No significant differences in views held by the groups were found between them, both for the actual and expected roles in these areas. ${ }^{86}$

\footnotetext{
${ }^{86}$ The ANOVA results in Table 37 and Table 44 in Appendices $\mathrm{G}$ and $\mathrm{H}$ showed no differences between the groups on the actual and expected roles, respectively, in physical facilities-related functions.
} 
Oliver's study had 30 items for actual roles and 30 items for expected roles. ${ }^{87}$ Silas had 30 items for actual roles and 42 items for expected roles. ${ }^{88}$ Their instruments did not have any specific question on the president's physical facilities-related functions. There was only one role expectation item that said, "The university and college president has educational development plan on paper" which could vaguely refer to physical facilities-related performances. The presidents, board members, and academic vice presidents in the Oliver and Silas studies agreed on many of the role items,-including the afore-mentioned function. Kim also had only two items, such as "Promote proper student respect for school property" and "Talk with staff for continuous physical improvement of the school." He found conflict between the board members and faculty members on these two items. $^{89}$

In literature, 12 percent of the president's time was thought to be sufficient for physical facilities responsibilities. ${ }^{90}$ In my study, however, the levels of performances and expectations in this category of functions would probably suggest higher percentages of the president's attention to facilities management compared to what is stated in the literature. This is so perhaps because in today's competitive educational offerings the campus needs to be attractive, with all its improved physical planning, buildings, classrooms, laboratories, dormitories, and other facilities, which the president is

\footnotetext{
${ }^{87}$ Oliver, 132-133, 135-136.

${ }^{88}$ Silas, $188-190,193-195$.

${ }^{89} \mathrm{Kim}, 66$.

${ }^{90}$ Corson, 12-25.
} 
ultimately responsible for. ${ }^{91}$ However the participants assessed the presidents' roles, there was a common consensus between them, for both the actual and expected roles of the presidents' physical facilities activities. ${ }^{92}$ This indicates cohesiveness among all the five groups of administrators in their perceptions and expectations.

\section{Community- and Alumni-Related Functions}

This section contained six items, 37-42. The statistical results presented in chapter 4 revealed a high level of consistency in agreement between the groups on all six items, both for actual and expected functions. The findings suggest that however the groups perceived the presidents' actual performances and however they expected the presidents' roles to be, there was a common consensus between the participating respondents to these role items. These roles are: assisting in fundraising activities; representing the institution to the churches; presenting reports about the college and university to the alumni; representing the institution to the public; associating with the alumni groups; and listening to the constituency. ${ }^{93}$

The groups were in harmony with one another in their levels of perceptions and expectations of these functions. There were no differences between them on these sets of actual and expected roles. These findings suggest that they were all in agreement about the way the presidents actually performed worldwide and the extent they were expected to

${ }^{91}$ Arthur Hansen, 1-7.

${ }^{92}$ Compare with Table 32.

${ }^{93}$ The ANOVA results presented in Table 38 in Appendix G and Table 45 in Appendix H showed no differences between the groups on the actual and expected roles, respectively, in presidents' communityand alumni-related functions. 
perform in public and alumni relations activities in all the SDA senior colleges and universities.

More than 30 years ago, Oliver, Silas, and Kim studied the perceptions and role expectations of the college and university presidents in the United States as perceived by three status groups. Oliver's instrument had a total of 60 questions for both actual and expected roles. Out of these, only one question was about the president's public and alumni relations activities. No differences in perceptions were found among the three groups surveyed on this item. ${ }^{94}$ Silas had two items on the public and alumni relations activities, among a total of 72 survey items on the actual and expected roles. He found no differences either in perceptions and expectations among the three status groups responding to these items. ${ }^{95}$ Kim's instrument had a separate section listing the public and alumni-related functions of presidents. This section had a total of eight questions. None of these items was found to be in conflict with any of the three groups in their responses to the presidents' role expectations on the public/alumni relations activities. ${ }^{96}$

Corson's summary of president's time spent in various activities allots 20 percent to public and alumni relations functions. ${ }^{97}$ The results of my study would perhaps suggest higher percentages for both the actual and expected roles in this category, based on the rankings of the seven categories of functions as perceived by the groups. The results

\footnotetext{
${ }^{94}$ Oliver, 90.

${ }^{95}$ Silas, 147.

${ }^{96} \mathrm{Kim}, 56-58$.

${ }^{97}$ Corson, 12-25.
} 
came on top, securing the first positions for both the actual and expected roles of the presidents' community- and alumni-related functions. ${ }^{98}$ These findings indicate that the groups are similar and in harmony. Their agreement regarding strong actual role performances and very high role expectations of the overall public relations responsibilities of the presidents is a conclusive indication of cohesiveness existing among all the status groups in the SDA senior colleges and universities around the world.

\section{Spiritual Life-Related Functions}

There were five variables in this section, 43-47. The results of this study revealed a high degree of consistency in agreement among all the groups surveyed regarding their perceptions and expectations of the presidents' actual and expected roles in the spiritual domain. This study shows that the groups are similar and in harmony with one another regarding their assessment as to how they perceived the presidents performed and how they expected them to perform the spiritual functions.

The spiritual functions the chief executive officers of the Seventh-day Adventist higher education institutions performed and were expected to perform are: promoting religious life of the students, faculty, and staff at the institution by delegating spiritual functions to campus chaplains and other personnel; taking ultimate responsibility in arranging and overseeing various religious programs in the institutions; manifesting Christ-like character in spiritual leadership roles; and focusing on the Christian philosophy of the institution. The groups did not differ from one another in their levels of

\footnotetext{
${ }^{98}$ See Table 57 in Appendix M. This table presents rankings of seven broad categories of actual and expected roles as perceived by the status groups, rank ordered by the means.
} 
perceptions and expectations of these role items. ${ }^{99}$

Oliver and Silas did not look into the spiritual dimension. Kim's research, limited to the SDA colleges and universities in the U.S.A., contained a separate spiritual section, in addition to seven other sections. This spiritual section had six questions on religious activities. Four of these were found to be in conflict between the board and faculty members. These items had to do with taking responsibility in chapels, convocations, vespers, and weeks of devotions; promoting religious life on campus; taking active part in church affairs; and making plans for students' religious activities. The presidents agreed among themselves regarding all of the six spiritual functions. ${ }^{100}$

More than 30 years after, I did not find any differences in my study between the groups on any of the spiritual functions mentioned in my questionnaire. No matter how the respondent groups viewed these spiritual activities, they were in consensus on how they perceived the presidents have actually carried out these functions in their work, and how they expected them to shoulder these responsibilities of a spiritual nature. This suggests cohesiveness among the board chairs, presidents, and vice presidents for academic, student life, and financial affairs in their perceptions of the spiritual functions the presidents actually carried out at their institutions. Since all the five respondent groups were Seventh-day Adventists and they were committed to the unique mission, ${ }^{101}$

\footnotetext{
${ }^{99}$ The ANOVA results presented in Table 39 in Appendix $\mathrm{G}$ and Table 46 in Appendix $\mathrm{H}$ showed no differences between the groups on the actual and expected roles, respectively, in the presidents' spiritual life-related functions. The five groups of respondents agreed among themselves on the degrees of how the presidents actually performed and how they were expected to perform in the spiritual life-related area.

${ }^{100} \mathrm{Kim}, 66,89$.

${ }^{101}$ Knight, 7-9.
} 
philosophy, ${ }^{102}$ and objectives ${ }^{103}$ of their institutions, they did not differ among themselves in their expectations either regarding the spiritual responsibilities of the presidents in the Adventist Christian institutions. The unique spiritual dimension makes the SDA senior colleges and universities distinct from the other centers of higher learning.

In summary, in light of the above discussion of Research Questions 3 and 4, I can conclude that Null Hypotheses 1 and 2 were true and tenable. There were no differences in perceptions and expectations or no probable role conflicts found between any of the five groups as to how the presidents were perceived to have actually performed and how they were expected to perform in the 47 role items examined. This cohesiveness among the groups, in my studied opinion, was due to the shared vision, mission, philosophy, and governance that the administrators and the governing bodies were committed to in the operation of the Seventh-day Adventist senior colleges and universities worldwide. It is very encouraging that this study did not show any non-congruousness among the responding administrators and their associates. This confirms that the presidency in general at the SDA senior colleges and universities around the world is on the target.

\section{Research Question 5}

Research Question 5 asked: Is there a difference in perception and expectation between actual role performances and expected roles of presidents of Seventh-day Adventist senior colleges and universities around the world, as perceived and expected by

\footnotetext{
${ }^{102}$ Fowler, $8,9$.

${ }^{103}$ Brown, 18 .
} 
their groups of board chairs, presidents, academic vice presidents, student service vice presidents, and finance vice presidents? This research question was answered with Null Hypothesis 3 for all null sub-hypotheses, 1-47, as presented in chapter 4 .

\section{Findings and Discussion of Null Hypothesis 3 in Relation to Research Question 5}

Null Hypothesis 3 stated that there is no difference between the perceived actual role performances and expected role responsibilities of presidents of Seventh-day Adventist senior colleges and universities around the world, as viewed by each of the status groups (board chairs, presidents, academic vice presidents, student service vice presidents, and finance vice presidents).

This null hypothesis was tested by two-tailed paired samples $t$-test to see if there were statistically significant differences between the perceived actual role performances and expected roles of the SDA senior college and university presidents. The results revealed that out of the 47 items examined, 16 of them showed no significant differences between each of the actual and expected roles. Therefore, these 16 items or null subhypotheses were true and tenable. The remaining 31 items showed significant statistical differences. Hence, these 31 items or null sub-hypotheses were false and untenable.

In the following pages of this section I have presented the findings of the statistical test results of Null Hypothesis 3 for all 47 paired items in relation to Research Question 5, based on Table 29 of chapter 4. This section discusses each of these 47 paired items under the seven broad categories of functions. These functions were organized into two sections, titled "Differences" and "No Differences" in perceptions and 
expectations between each pair of actual role and expected role. Supported by the literature as much as possible, the following discussion attempts to find the implications that could be attached to the findings.

\section{Student-Related Functions--} Actual versus Expected

No differences

The findings of this study indicate that there were no significant statistical differences between the perceived actual role performances and expected roles of the presidents for the following four student-related items:

\section{Participate in student recruitment}

5. Take ultimate responsibility for student discipline

6. Teach some classes in order to maintain contact with students

7. Know every student personally.

The above four items of Null Hypothesis 3 were retained. The overall findings suggest that the presidents did almost as much as they should in these functions. They seemed to have delegated all the student-related matters to the vice presidents for student life, dorm deans, and other officers, as specified in their faculty handbooks and emphasized in the literature. ${ }^{104}$

Item 1: As regards recruiting students, there were no significant differences found between the actual and expected roles. The presidents seemed to have acted upon this particular job fairly well as expected. They must have delegated much of this

${ }^{104}$ Maphosa, in Christ in the Classroom, (1994), 12:151. 
responsibility to a team of professional personnel, as McAllister-Wilson, Williford, and Neelands have proposed. ${ }^{105}$ However, the findings also indicate that the presidents were ultimately responsible for overseeing the promotion of the student recruitment plans ${ }^{106}$ based upon the mission, philosophy, educational program, and budgetary provision of the institutions.

Item 5: According to Brown, ${ }^{107}$ student discipline is part of the Seventh-day Adventist college and university presidents' responsibility. The findings suggest that the presidents performed this responsibility as closely as expected. In many countries, I know that in private schools, colleges, and universities there exists "no right to due process in student discipline" 108 that can be challenged in the court of law. Overall, the presidents were not expected to do a significant improvement in this area. ${ }^{109}$ However, all policies need to be made known in written form and the issues should be settled through legal counsel of the institution."110

Item 6 had to do with the presidents teaching classes. There was no significant difference between this perceived actual role and expected role. This is perhaps due to

${ }^{105}$ McAllister-Wilson, Williford, and Neelands, in Lewis and Weems, Jr., 144.

${ }^{106}$ Brown, 66, sec. 2, P.

${ }^{107}$ Ibid., 66, sec. 2, Q. Also, see Table 32.

${ }^{108}$ Lyndon G. Furst and Charles J. Russo, The Legal Aspects of Nonpublic Schools: A Casebook (Berrien Springs, MI: Andrews University, 1993), 383-391. While discussing a case study, Furst and Russo mentioned a court's decision to deny the application of the Fourteenth Amendment to the disciplinary procedures of private colleges and universities in the United States.

${ }^{109}$ See Table 32.

${ }^{110}$ C. K. Gunsalus, The College Administrator's Survival Guide (Cambridge, MA: Harvard University Press, 2006), 161-166. 
the fact that in many large institutions the presidents could not do regular formal teaching in the classroom setting nor were they expected to make a big shift in their attention to this role because of their multiple responsibilities.

Item 7 was about knowing every student personally. The perceived actual role performance of this item did not differ significantly from the role expectation of the groups. The finding reveals a low expectation regarding knowing every student personally. This is not possible in large institutions. Worldwide, the SDA college and university presidents, I would say, were found to be doing as much as they probably could in this area.

Bouchard, Thistlethwaite, and Weber believed that classroom teaching is a valued task that provides the presidents a highly effective way of staying in touch with the students. ${ }^{111}$ Among many other college/university chief executive officers, President Prince of Hampshire College understood the value of knowing students personally through formal class teaching. ${ }^{112}$ It is true that many college and university presidents worldwide have taught and still probably teach in the classroom settings. ${ }^{113}$ In his address, President Jenkins of the University of Notre Dame mentioned that he enjoyed

${ }^{111}$ Charles E. Bouchard, Susan Thistlethwaite, and Timothy Weber, in Lewis and Weems, Jr., 81.

${ }^{112}$ Gregory S. Prince, Jr., Teach Them to Challenge Authority: Educating for Healthy Societies (New York, NY: The Continuum International Publishing Group, 2008), 204-216. Prince served 16 years as president of Hampshire College.

${ }^{113}$ I have been to some overseas SDA college and university campuses in Bangladesh, Hong Kong, India, Indonesia, Pakistan, Philippines, Singapore, South Korea, Sri Lanka, and Thailand. I have come to know that the SDA college/university presidents in these countries taught classes. In North America I know that some presidents, particularly in smaller SDA colleges, have taught academic courses on a regular basis in the classroom settings. I would imagine that in some institutions in other world divisions it was possible for the SDA college and university chief executive officers to do the same. 
teaching classes to know the students better. ${ }^{114}$ No matter how the presidents have performed worldwide on the above four items, $1,5,6$, and 7 , the findings suggest that they were not expected to make a significant change in their performances of these roles.

\section{Differences}

The remaining four student-related items showed significant statistical differences between the perceived actual role performances and expected roles of the presidents as the following present:

\section{Work closely with student government leaders}

\section{Dialogue frequently with students}

\section{Visit students periodically in the dorms during dorm worships}

\section{Serve as mentor of students.}

The above four items of Null Hypothesis 3 were rejected. The findings suggest that the SDA senior college and university presidents were not doing enough in the above 50 percent of the student-related functions worldwide. This study shows that they were expected to perform better than what they were perceived doing.

Items 2,3 , and 4: The literature and views from the college and university presidents provide references to working and dialoguing with the students and visiting them in their residence halls. ${ }^{15}$ Among others, Prince has shared his experience of

${ }^{114}$ Rev. John I. Jenkins, "The Role of a Catholic President in the $21{ }^{\text {st }}$ Century: A Letter from the President," www.controller.nd.edu/files/reports/2006-president.pdf (accessed April 2, 2008). In his letter to the members of the Notre Dame family for the academic year ended June 30, 2006, Rev. Jenkins, president of the University of Notre Dame, South Bend, Indiana, mentioned that he took his classroom teaching as an opportunity to become aware of the students' academic needs and their personal concerns.

${ }^{115}$ Fisher and Koch, 168. 
dialoguing, working, and introducing the Monday breakfast meetings with the students. These approaches gave him answers to many problems. ${ }^{116}$ Fisher and Koch also recommended building and maintaining strong relationships with the students by working with the student government leaders, listening to them, and visiting the residence halls. ${ }^{117}$ The findings of this study suggest that the presidents need to pay more attention to these areas through the student service vice presidents and dorm deans so that the expectation levels could be met.

Item 8: Serving as mentor of students showed the highest difference in the groups' perceptions between the actual and expected roles among all the other pairs in this section. ${ }^{118}$ However, it does not mean that the presidents should directly involve themselves too much into the mentoring details. The student success program or student intervention program carried out on many campuses can function in a number of ways. Val Tarbitt's case studies on mentoring justifies the personal interactions with the students to be more effective than on-line exchanges. ${ }^{119}$ Elden Kamwendo has also emphasized the president's mentoring role. As mentors, the presidents can foster in the students the values of love, unity, justice, integrity, honesty, and prudence. ${ }^{120}$

${ }^{116}$ Prince, 3-23.

${ }^{117}$ Fisher and Koch, 157-168.

${ }^{118}$ See Table 29, \#8.

${ }^{119}$ Val Tarbitt, "Mentoring On-Line: Rethinking the Tutor/Student Experience," quoted in Cedric Cullingford, Mentoring in Education: An International Perspective (Burlington, VT: Ashgate Publishing, 2006), p. xv, 183-203.

${ }^{120}$ Elden G. Kamwendo, "The Role of Administrative and Support Units of an Adventist University in Fostering Integration of Faith and Learning," in Christ in the Classroom, (2001), 28:142. 
According to the survey conducted by Ila Weeks, roughly 18 percent of the president's time was spent in educational matters that included, among other items, "meeting with student groups and student leaders and handling, with his staff, student problems." 21 It is understood that in the twenty-first century this percentage may vary from institution to institution. In any case, the presidents' student-relations activities have always been of great importance in all institutions. In 2006, McAllister-Wilson, Williford, and Neelands stated that the students are the school's mission, or at least are the primary means by which a college accomplishes its mission. ${ }^{122}$ Also, Hesburgh and Kerr expected the university president to be a friend of the students. ${ }^{123}$ Their needs and their concerns, therefore, should be considered with highest priority. ${ }^{124}$

The results of this study revealed that among the seven broad categories of functions, the overall student-related roles became seventh in rank order of the presidents' perceived actual performances and expected responsibilities. ${ }^{125}$ It also showed that the perceived actual performances and role expectations of the presidents in the studentrelated area were the lowest among all the activities listed under the seven principal dimensions of functions. Even though the perceptions and expectations were not

\footnotetext{
${ }^{121}$ Ila D. Weeks, "The University President and the Publics," presidential address, National Association of State Universities Transactions and Proceedings, 1950, quoted in Corson, 12-25.

${ }^{122}$ McAllister-Wilson, Williford, and Neelands, in Lewis and Weems, Jr., 141-142.

${ }^{123}$ Douglas Covington, "Forging a Unified Vision Among Diverse Constituencies," in The Advancement President and the Academy: Profiles in Institutional Leadership, ed. Mary Kay Murphy (Phoenix, AZ: Oryx Press, 1997), 99. Covington, was president of the Radford University, Radford, VA, when he quoted Father T. M. Hesburgh and Clark Kerr.

${ }^{124}$ Ibid., 104.

${ }^{125}$ See Table 57, sec. I in Appendix M.
} 
remarkably higher, the findings of this study do clearly indicate that the presidents are expected to maintain closer relations with the students than their perceived actual performances. They can accomplish this primarily through the vice presidents of student life, dorm deans, academic advisors, and professors who deal with the students more directly on a daily basis. Duane Acker's experience as president of the Kansas State University is worth noting. He maintained a strong relationship with the students. ${ }^{126}$

\section{Employee-Related Functions-- Actual versus Expected}

No differences

The results of this study revealed that there were no significant statistical differences between the perceived actual role performances and role expectations of the presidents for the following five employee-related items:

9. Participate in faculty recruitment

10. Serve as academic leader of faculty

12. Have responsibility in submitting proposed changes in academic matters to faculty for approval

15. Collaborate for advanced in-service training for faculty and staff

16. Carry out policies and regulations of faculty and staff.

The above five items of Null Hypothesis 3 were retained. The findings tend to

\footnotetext{
${ }^{126}$ Duane Acker, Can State Universities Be Managed? A Primer for Presidents and Management Teams (Westport, CT: Praeger Publishers, 2006), 174-176. Acker is President Emeritus of Kansas State University. He also served at four other higher education campuses in various administrative positions. His long years of experience helped him understand the importance of building and maintaining stronger student-relations with the college/university administration.
} 
suggest that the presidents were not expected to do much more than what they were actually seen doing in each of the above roles. They seemed to have carried out their duties as nearly as expected in the areas of faculty recruitment; academic leadership; inservice training for employees; and implementing policies and regulations for faculty and staff. These roles were mentioned in the institutions' faculty manuals, ${ }^{127}$ Brown's volume, ${ }^{128}$ and literature which I have examined.

Item 9: As regards recruiting faculty, the findings of this study lead me to assume that the presidents have done this job fairly well, meeting the expectation level of the board chairs and on-campus support administrators. As pointed out by Narbarte, ${ }^{129}$ the presidents of the SDA senior colleges and universities must have been guided by the unique mission, philosophy, objectives, and budgetary provision, while carrying out this particular job. The General Conference Education Department of the Seventh-day Adventist world church has also made an almost similar recommendation regarding recruitment of faculty and staff who are committed to the message, mission, and lifestyle, and to the education of young men and women for a useful life "in this world and in the world to come."130

Item 10: Providing academic leadership was another activity that was seen to have

\footnotetext{
${ }^{127}$ Faculty handbooks, operating manuals, and by-laws from 27 colleges and universities in 14 countries were examined by me. These operating manuals listed the presidents' duties in the area of faculty and staff administration.

${ }^{128}$ Brown, 65-66.

${ }^{129}$ Miriam P. Narbarte, "Teaching Human Resources Management: Its Implications for Seventhday Adventist Educators," in Christ in the Classroom, (1994), 2:101-102.

${ }^{130}$ See Appendix D, sec. 4.
} 
performed moderately and was expected to exhibit a moderate performance as well. The findings suggest that the presidents were not expected to get involved in the minutiae of the academic affairs of the institutions, but to continue to be the "final authority in the academic mission. ${ }^{\prime 131}$ The literature and survey reports emphasized the importance of the academic responsibilities. In his article in 1998, Rhodes referred to the Report of the Commission on the Academic Presidency published in 1996 that urged the delegation of more academic authority to the president. According to him, "if universities are to prosper, the presidents' academic leadership is desirable." ${ }^{132}$ Duderstadt, who served as president at the University of Michigan, engaged himself in academic leadership. ${ }^{133}$

Item 12: The presidents' actual practice in proposing academic changes was in the same degree of expectation by the groups. The presidents' actual performances met the expectations. The responses tend to make me assume that the presidents did not have to and were not expected to propose a lot of academic changes, since much of the academic matters were shouldered by the provosts, or academic vice presidents, or academic deans as per the job descriptions I have examined. However, the presidents, I know, were aware that "as chief executives, they were responsible for its implementation." 134

${ }^{131}$ Bouchard, Thistlethwaite, and Weber, in Lewis and Weems, Jr., 73.

${ }^{132}$ Frank H. T. Rhodes, "The University at the Millennium: Missions and Responsibilities of Research Universities," in Governance in Higher Education: The University in a State of Flux, ed. Werner Z. Hirsh and Luc E. Weber (London, England: Economica, 2001), 12-13.

${ }^{133}$ James J. Duderstadt, The View from the Helm: Leading the American University During an Era of Change (Ann Arbor, MI: The University of Michigan Press, 2007), 166-172.

${ }^{134}$ Maphosa, 148; see also Alan E. Guskin and Mary B. Marcy, "Pressures for Fundamental Reform: Creating a Viable Academic Future," in Field Guide to Academic Leadership, ed. Robert M. Diamond (San Francisco, CA: Jossey-Bass, 2002), 3-14. 
Item 15: Employee training was another responsibility that the presidents' perceived actual performances were not significantly lower than the respondents' expectation level. The presidents seemed to have demonstrated in their practices a faculty development initiative which was implemented on an ongoing basis. The presidents, I am sure, believed that "faculty upgrading is an important investment"135 and would help prepare the employees to participate in the shared governance. ${ }^{136}$

Item 16: The institutions' policies and regulations pertaining to faculty and staff were also carried out well as expected. The findings suggest that the presidents were convinced that the policies, customarily approved by the governing boards and also the other regulations that are internally enforced by the presidents, should be organized in one or more handbooks and given to each employee. Hansen, Stairs, and Weeks maintained that "the president can delegate this task to an institutional colleague, but the president needs to see that this is done, ${ }^{\prime 137}$ since it is the president's ultimate responsibility to participate in the development and implementation of the institutional policies. ${ }^{138}$

\section{Differences}

The remaining three items of the employee-related functions showed significant

${ }^{135}$ Maphosa, in Christ in the Classroom, (1994), 12:151.

${ }^{136}$ David Birkenstock, "Leadership: The Key Dimension in Adventist Tertiary Educational Administration," in Christ in the Classroom, (1994), 12:45. Birkenstock served as president at the Adventist International Institute of Advanced Studies in the Philippines.

${ }^{137}$ Adolf Hansen, Jean Stairs, and Kent M. Weeks, "The President's Role in Administration and Personnel Management," in Lewis and Weems, Jr., 23.

${ }^{138}$ Norma M. Goonen and Rachel S. Blechman, Higher Education Administration: A Guide to Legal, Ethical, and Practical Issues (Westport, CT: Greenwood Press, 1999), 183. 
statistical differences between the groups' perceived actual performances and expected roles of the presidents as the following present:

\section{Guard faculty's academic freedom}

\section{Work with faculty in reviewing accrediting agency's reports}

\section{Recommend to board for hiring, promotion, demotion and dismissal of}

faculty and staff.

The above items of Null Hypothesis 3 were rejected. The significant statistical differences found between the perceived actual and expected roles suggest that the presidents were not meeting the expectations of the respondents in their performances of the above responsibilities. The presidents were expected to step up their efforts to increase the discharge of these duties.

Item 11: The presidents did not meet the expectations of guarding the academic freedom of the faculty. Several factors may have contributed to the shortfall in the actual performance of this particular job. First, there may not have been academic freedom in place in many of the overseas institutions, as Akker noted. ${ }^{139}$ Second, the colleges and universities in some parts of the world may have a different notion about the academic freedom with no boundaries, as feared by Keohane. ${ }^{140}$ Third, a church institution may not be sensitive to issues of academic freedom, as observed by Cushing, Morgan, and

${ }^{139}$ John Akker, "Protecting Academic Freedom Worldwide," ACADEME 88, no. 3 (2002): 44-45. Akker noted that in many parts of the world there is no academic freedom in the educational institutions.

${ }^{140}$ Nannerl O. Keohane, Higher Ground: Ethics and Leadership in the Modern University (Durham, NC: Duke University Press, 2006), 250-252. Keohane, who served 24 years as president at Wellesley College and Duke University, believed that academic "freedom must always have boundaries that should be cautiously erected and carefully observed" (p. 250). 
Aymer. ${ }^{141}$ Fourth, the SDA colleges and universities, being religious campuses, did not and still do not, perhaps, adopt the 1940 AAUP standards per se because these institutions believe that their mission to transmit a particular religious position is a stronger value than that of the traditional academic freedom. ${ }^{142}$ According to Gallimore, academic freedom in the SDA institutions of higher education is viewed in the context of faith in God and His divine revelation in Scripture. ${ }^{143}$ Fifth, the SDA college/university presidents may not have involved themselves in this role item as expected, because the provosts or academic vice presidents were vested with this responsibility. However, the Seventh-day Adventist world church acknowledges academic freedom in SDA higher education to pursue knowledge and truth within the confines of the church's fundamental beliefs. ${ }^{144}$ If efforts are made for such pursuit of "knowledge and truth," as recommended by the General Conference of the SDA world church, differences will be narrowed down.

Item 13: The presidents' work relating to the accrediting agency's reports showed a significant difference between the actual and expected roles. Cushing, Morgan, and Aymer made some suggestions to improve on this function. According to them, the presidents should have a comprehensive understanding of the standards and regulations of

\footnotetext{
${ }^{141}$ Vincent Cushing, Donn Morgan, and Albert Aymer, "The President's Role with External Authorities," in Lewis and Weems, Jr., 162.

${ }^{142}$ Goonen, 135 .

${ }^{143}$ Jay Gallimore, "Evolution in Education?" Michigan Memo: Official Newsletter for the Michigan Conference of Seventh-day Adventists 21, no. 5 (2009): 2.

${ }^{144}$ Bert B. Beach, "Can a University Be Christian?: A Look at Academic Freedom on the Adventist Campus," Adventist Review 166, no. 9 (1989): 19-24. In this general paper of the Seventh-day Adventist Church, Beach quoted the Seventh-day Adventist world church's 1987 official position that "acknowledges academic freedom" of the teachers "to pursue knowledge and truth" within the confines of the fundamental beliefs of the church.
} 
the accrediting agencies. They should foster amicable relations and implement the guidelines and requirements recommended by the accrediting organizations. ${ }^{145}$ Although the various disciplines' academic deans, institutions' academic vice presidents or provosts may have a more direct responsibility to attend to much of the agency's reports, the presidents, who, according to many college/university chief executives I have read about in the literature, are ultimately responsible in reviewing and implementing the accrediting agency's recommendations, since they are the academic leaders. ${ }^{146}$

Item 14: This item had to do with the presidents' roles in hiring, promotion, demotion, and dismissal of employees. Statistically, this item showed a significant difference between the perceived actual performances and expected roles. The findings suggest better role expectations than what the presidents demonstrated in their actual practices. This role item has several sub-items, each of which has several steps that need to be followed before a final decision can be made. The SDA working policy and the institutions' faculty handbooks or operating manuals have clearly stipulated the criteria for selection, hiring, compensation, and termination of faculty and staff. The descriptive data indicate that the SDA college/university presidency under the guidance of the governing boards has been doing a fairly good job worldwide in this particular role. ${ }^{147}$ However, the presidents were expected to do even better. In the literature, researchers

${ }^{145}$ Cushing, Morgan, and Aymer, in Lewis and Weems, Jr., 162-163.

${ }^{146}$ Francis L. Lawrence, Leadership in Higher Education: Views from the Presidency (New Brunswick, NJ: Transaction Publishers, 2006), 245. Lawrence interviewed 13 college and university presidents. In answer to one of Lawrence's questions, Nil Hasselmo, former president of the University of Minnesota, affirmed that the presidents are the academic leaders of their institutions.

${ }^{147}$ See Table 32, \#14. 
and scholars have offered some suggestions to improve in this area. Bouchard, Thistlethwaite, and Weber have stated that the president may or may not be actively involved in specific faculty searches, but he or she should have a vision for the institution that would influence hiring of personnel. ${ }^{148}$

As regards promotion, the size and type of SDA colleges and universities, like any other religious institutions, according to Hansen, Stairs, and Weeks, seem to limit the opportunities for an upward mobility of the employees within their institutions. ${ }^{149}$ In Christian institutions, promotion should not be seen as a result of working hard, but be viewed as the result of God's direction to the employee. ${ }^{150}$ However, the findings of this study could imply that when opportunities come, the presidents should do more in granting the deserving employees higher positions or salary increases based on annual performance appraisals and institutions' policies.

The presidents' handling of faculty/staff demotion and dismissal, which, to my observation, are not very common in the SDA colleges and universities, may suggest that the presidents, in collaboration with the boards, need to step up efforts to "ensure that the disciplinary actions are based upon transparent guidelines and principles." ${ }^{.151}$ and the chief executives must be aware of the procedures. ${ }^{152}$ This would help reduce the gap between

\footnotetext{
${ }^{148}$ Bouchard, Thistlethwaite, and Weber, in Lewis and Weems, Jr., 14, 15.

${ }^{149}$ Hansen, Stairs, and Weeks, in Lewis and Weems, Jr., 28.

${ }^{150}$ Narbarte, in Christ in the Classroom, (1994), 2:107

${ }^{151}$ Hansen, Stairs, and Weeks, in Lewis and Weems, Jr., 29.

${ }^{152}$ Goonen, 185 .
} 
the perceived lower performances and higher expectations of the presidents' handling of demotion and dismissal of faculty/staff issues.

\section{General Administrative Functions-- Actual versus Expected}

No differences

This study shows that there were no significant statistical differences between the perceived actual performances and role expectations of the SDA senior college and university presidents for the following two items of the general administrative responsibilities:

17. Prepare agenda for board of trustees' meetings

18. Preside over general faculty meetings.

The above items of Null Hypothesis 3 were retained. The overall findings suggest that the presidents' perceived actual performances in the areas of agenda preparation for board meetings, and chairing of general faculty meetings in their institutions were in the same expectation level of the respondent groups. The presidents performed as expected in these two general administrative functions.

Item 17: The results of this study indicate that the chief executive officers of the Seventh-day Adventist colleges and universities around the world were doing as much as they were expected to do in the area of closely working with the trustees, presenting all relevant matters to the boards for necessary action. The operating manuals and faculty handbooks that included the job descriptions of the various officials of the institutions have specifically mentioned the president's responsibility of presenting the items to the 
board as board secretary. ${ }^{153}$ Brown's official handbook for the General Conference Education Department of the Seventh-day Adventist world church lists the duties of the president in relation to the board. ${ }^{154}$ Fisher and Koch indicated that it is the president's duty to include items on the agenda for the board meetings and executive sessions. ${ }^{155}$ Cooley, Klein, and Weeks recommended that the agenda items and reports be sent to the chair and members of the trustees ahead of time. ${ }^{156}$ The findings suggest that the presidents worldwide have followed all this and so their actual performance of this role, which ranked second among all the items, ${ }^{157}$ met the expectations of the respondents.

Item 18: This item had to do with presiding over the general faculty meetings of the institutions. The findings tend to indicate that the presidents met the expectations of the respondents for this particular job. There was no significant difference in perceptions between how the presidents actually performed and how they were viewed to perform this responsibility. All across the Seventh-day Adventist senior colleges and universities around the world there seemed to have an unanimity of opinions in this regard. The results reveal that the perceived actual performance of this role is the closest to the degree of expectation of this role item among all the 47 items. $^{158}$ This is a very good picture. It

\footnotetext{
${ }^{153}$ I have examined 27 faculty manuals of Catholic, Protestant, Adventist, and Ivy League colleges and universities. Copy of this list is placed in my personal file.

${ }^{154}$ Brown, 65 .

${ }^{155}$ Fisher and Koch, 242-243.

${ }^{156}$ Cooley, Klein, and Weeks, in Lewis and Weems, Jr., 46-47.

${ }^{157}$ See Table 25 in chapter 4.

${ }^{158}$ See Table 29 in chapter 4 and compare the $t$-value of \#18 with all other $t$-values in the table. The very small difference between the actual and expected role is negligible.
} 
is not unlikely that the broad concept of Fisher and Koch for healthy faculty meetings may have been adapted by the presidents in the SDA senior colleges and universities. It was recommended that no more than one general faculty meeting in a given semester be conducted to keep the faculty informed about the major initiatives of the institution and to listen to everyone. Two general faculty meetings a year should be enough, one of which could have the "State of the Institution" address by the president. No other general faculty meetings need to be called except during periods of emergency..$^{159}$ The findings suggest that the presidents did an excellent job in this area following some kind of a wellcommunicated practice and everybody seemed to be satisfied.

\section{Differences}

The remaining seven items of the general administrative functions showed significant statistical differences between the perceived actual performances and role expectations of the presidents as the following present:

19. Take responsibility in structuring student admission standards

20. Take responsibility in strategic planning for institution

21. Take a leading role to prepare, revise, and follow organizational chart

22. Foster research and intellectual climate for institution

23. Comply with legal mandates and laws in addressing grievances

24. Help formulate and adhere to institution's educational philosophy

25. Delegate responsibility, authority, and needed resources to administrators. had conducted.

${ }^{159}$ Fisher and Koch, 149-150, 156. The authors' views were based on the results of a study they 
The above items of Null Hypothesis 3 were rejected. These items constitute 78 percent of the general administrative functions that showed significant statistical differences between the actual and expected roles. It means that almost four fifths of the total number of items in this category were lagging far behind the expectations of the participants who responded. The findings suggest that the presidents need to improve their performances in these seven role items.

Item 19: The findings suggest that the respondents expected much more involvement of the presidents in matters of student admissions than what they were found actually doing in this area. The presidents may have delegated this responsibility to the public relations directors, alumni officers, and admissions office personnel, as Fisher and Koch, in their observation, have seen done in colleges and universities. ${ }^{160}$ In many cases, especially in large institutions, the presidents may have totally depended upon the public relations, alumni, and enrollment officers regarding this responsibility. The presidents may not have bothered to engage themselves in looking into the student admission standards and enrollment activities. McAllister-Wilson, Williford, and Neelands noted that the presidents often see fundraising as their most important role, neglecting to pay more attention to student enrollment. According to them, students are the school's mission, or at least are the primary means by which the institutions accomplish their mission. ${ }^{161}$

Item 20: Strategic planning was another item of the general administrative

\footnotetext{
${ }^{160}$ Fisher and Koch, 224-225.

${ }^{161}$ McAllister, Williford, and Neelands, in Lewis and Weems, Jr., 141-143.
} 
responsibilities that the presidents were found to be trailing far behind the expectation mark. ${ }^{162}$ The statistical results showed a notable difference between the perceived actual performance and role expectation of this particular responsibility. The findings suggest that the presidents should engage themselves more in the strategic planning for their institutions than their perceived level of actual performances. The respondents of this study, I would say, were in agreement in principle with Cooley, Klein, and Weeks who have considered strategic planning with the faculty to be one of the major tasks of the presidents. According to them, the presidents should focus the institutions' energies upon the future, engaging themselves in the strategic planning process to implement the statement of mission in such a way that it becomes a vision for success. ${ }^{163}$ In all their routine functions ${ }^{164}$ the future of the institutions should be their highest priorities. ${ }^{165}$ The presidents are the leaders of strategic planning for program development of their institutions. ${ }^{166}$ The other objectives of the strategic planning, according to Wileman, are to enhance quality, optimize student profile, strengthen research engagement, maintain financial sustainability, maintain faculty/staff quality, and maintain and develop a supporting infrastructure and the environment. ${ }^{167}$ The SDA college and university

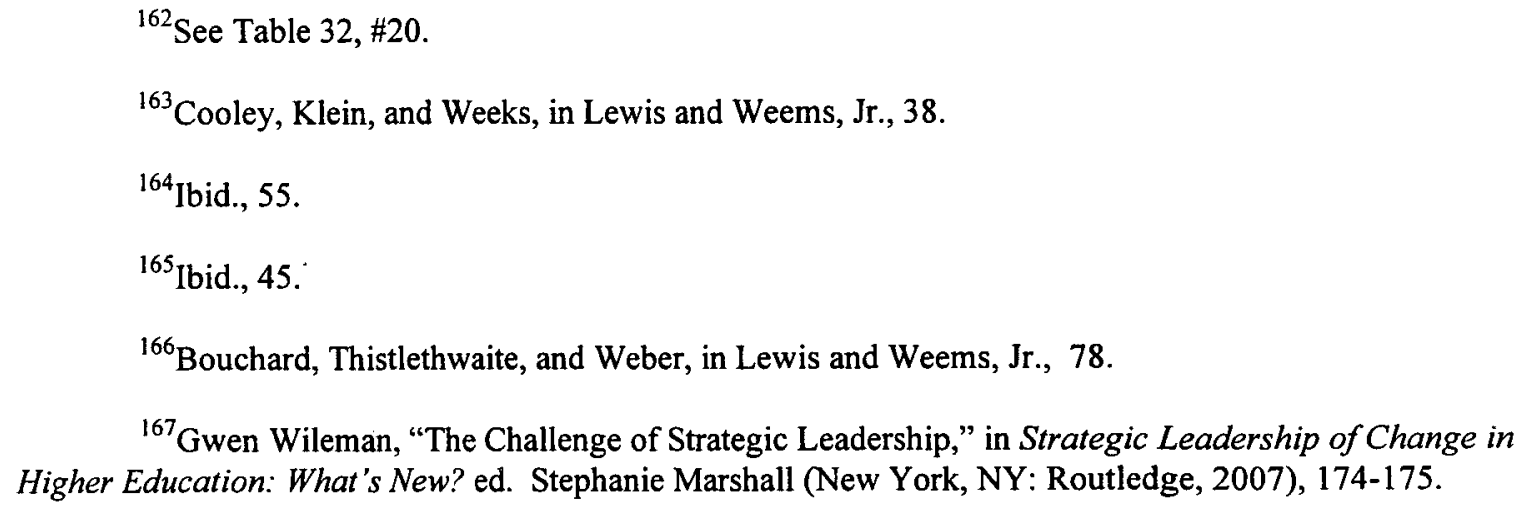
Higher Education: What's New? ed. Stephanie Marshall (New York, NY: Routledge, 2007), 174-175. 
presidents are expected to pay more attention to the all-around strategic planning activities to meet the mission of the institution and to face the changing environment.

Item 21: The presidents' actual performances in taking a leading role in the preparation, revision, and implementation of their institutions' organizational charts were not found to be up to the expectations of the research participants. Although the presidents did a good job on this, they were expected to do better. ${ }^{168}$ The results of this study indicate that the presidents need to give more attention to the "organizational chart that is often used to visualize the total organization, including major functions, relationships of positions, lines of authority and communication, relative authority and power, channels of supervision, and other general organizational patterns," $" 169$ as Cunningham and Cordeiro have stated. The findings suggest that many of the SDA colleges and universities around the world need to specify the various components of a classical organizational structure to avoid possible role conflict and role ambiguity. ${ }^{170}$

Item 22: This item had to do with fostering research and intellectual climate for the SDA senior colleges and universities. The statistical results yielded from the participants' responses reveal that the presidents were not doing enough in this area. The finding of this study suggests that the president should pay more attention to ensure that intellectual climate is ever-present on the campus and that the faculty members engage

\footnotetext{
${ }^{168}$ Compare the actual level with expected level of item 21 in Table 32.

${ }^{169}$ William G. Cunningham and Paula A. Cordeiro, Educational Leadership: $A$ Bridge to Improved Practice, $4^{\text {th }}$ ed. (Boston, MA: Pearson Education, 2009), 301.

${ }^{170}$ I have been to many college and university campuses overseas where 1 have sensed the uncertainty of roles of various unit administrators, as faced and expressed by the faculty and students. This role ambiguity could cause role conflict among the upper level administrators in the educational institutions.
} 
more and more in research within the confines of the institution's "mission, purpose, and moral values."171 Johnson's stance is supportive of this finding. He stated that "higher education should devote time and assets to research because research is an essential mission of higher education," ${ }^{\prime 172}$ and according to Payton, one of the purposes of higher education is research. ${ }^{173}$ I believe the findings of this study would imply that the members of the faculty are expected to engage themselves more in research projects, debates, forums of discussion, and such other intellectual exercises, keeping the mission of the institution at the focal point. The president, as Steven Muller termed it, "serves as the chief persuader," trying to persuade the faculty to do research and publishing ${ }^{174}$ that could help advance knowledge and improve scholarly performance. ${ }^{175}$

Item 23: This study shows a significant statistical difference between the degrees of actual perceived performance and role expectation in the president's work of complying with legal mandates and laws in addressing grievances of the employees and students. According to the findings, all participating groups' perceptions indicate that the

${ }^{171}$ Robert L. Payton, "Presidents as Public Teachers," in The Advancement President and the Academy: Profiles in Institutional Leadership, ed. Mary Kay Murphy (Phoenix, AZ: Oryx Press, 1997), 9. Payton served at the Indiana University-Purdue University, Indianapolis, Indiana.

${ }^{172}$ Joseph E. Johnson, "State Universities, Legislative Constituencies, and the Advancement President," in The Advancement President and the Academy: Profiles in Institutional Leadership, ed. Mary Kay Murphy, 85. Johnson served as president of the University of Tennessee, Knoxville, Tennessee.

${ }^{173}$ Payton, quoted in Murphy, 8; see also Stephen H. Davis, "Bridging the Gap Between Research and Practice: What's Good, What's Bad, And How Can One Be Sure?" Phi Delta Kappan 88, no. 8 (2007): 569-578.

${ }^{174}$ Steven Muller, "The Advancement Role in the Research University," in The Advancement President and the Academy: Profiles in Institutional Leadership, ed. Mary Kay Murphy, 49. Muller is the president emeritus of The Johns Hopkins University, Baltimore, Maryland.

${ }^{175}$ Payton, quoted in Murphy, 6. 
presidents are currently doing good in this particular job. It could be that there may not have been too many grievance cases in the Seventh-day Adventist higher education institutions worldwide requiring legal attention. My personal experience and knowledge about many campuses make me assume that many effective executives, as Maphosa, an SDA scholar and higher education administrator, has pointed out, follow a systematic process in identifying and solving problems ${ }^{176}$ within their institutions that can avoid legal issues in addressing employee and student grievances. The findings also suggest that the presidents need to exceed their actual performance level in this particular area. One of the ways to achieve this expectation, as Hansen, Stairs, and Weeks ${ }^{177}$ and $B r o w n{ }^{178}$ have suggested, is to establish formal procedures for an employee to file a grievance and review grievances regularly with the personnel. The president needs to retain an impartial stance and seek to resolve the grievances in as informal a manner as possible.

Item 24: Formulating and adhering to an institution's educational philosophy is another function that shows a significant statistical difference in perceptions between the presidents' actual and expected performances. The findings suggest that the presidents of the SDA senior colleges and universities worldwide performed this job well but they were expected to do better. ${ }^{179}$ The presidents' expected performance of this role occupies a second position, a very high expectation indeed, by rank order of the groups' total

${ }^{176}$ Maphosa, in Christ in the Classroom, (1994), 12:153.

${ }^{177}$ Hansen, Stairs, and Weeks, in Lewis and Weems, Jr., 31.

${ }^{178}$ Brown, 127-128.

${ }^{179}$ See Table 32, sec. III, \#24. The expected level is higher than the actual level. According to the respondent groups, the presidents should perform this responsibility to the highest level of expectation. 
expected mean score. ${ }^{180}$ This is not surprising because in every area of leadership function, the educational philosophy of the institution should be the guiding force, more so in the Seventh-day Adventist centers of higher education that are classified as Christian institutions whose philosophy is distinctive of all other "secular institutions," as Folkenberg indicated. ${ }^{181}$ Noftzger also noted some distinct features of church-related institutions. ${ }^{182}$ According to Aleshire, Campbell, and Mannoia, educational philosophy gives direction for leadership. It not only directs and stabilizes the work of a school, it also grounds the president's work and provides renewal along the way. ${ }^{183}$ It is, therefore, understandable why the status groups had a very high expectation of this role item.

Item 25: The statistical results reveal that delegation of responsibilities, authorities, and resources was most lagging behind the groups' expectations among all the general administrative functions. ${ }^{184}$ This item ranked third among all 47 items in terms of highest difference in perceptions between actual performance and expected role of the presidents. The findings suggest that the presidents need to improve much more in

${ }^{180}$ See Table 27, Level 1. The respondents' degree of expectations for this role item averaged in to the second highest score among the multiple statistics for both actual and expected means.

${ }^{181}$ In his keynote address at the 1992 Annual Council, Robert S. Folkenberg, Sr., former president of the Seventh-day Adventist world church, reminded the delegates of the educational philosophy of the SDA educational institutions. Quoted in Geraty, 5.

${ }^{182}$ Geraty, 10. In his article Lawrence Geraty quoted Richard Noftzger's views on church-related institutions that ought to be different from public and other private institutions in quality and emphasis, in both theory and practice, in the mission statement and in institutional culture, in the curriculum and in the expected conduct of students, in the projected majors and vocations of students and in the shared commitments of Christian vocation, and in administrative policies and classroom teachings.

${ }^{183}$ Daniel Aleshire, Cynthia Campbell, and Kevin Mannoia, "The President's Vocation and Leadership," in Lewis and Weems, Jr., 7-8.

\footnotetext{
${ }^{184}$ See Table 29.
} 
this area. All the participants in this study seem to know that the college and university presidents' roles are very complex in the twenty-first century ${ }^{185}$ and that there is no alternative to delegation of responsibilities, for it can bring effectiveness in the total operation of the institution. ${ }^{186}$ When asked about the secret of her success at the University of Iowa and University of Michigan, President Mary Coleman said that she delegated responsibilities to the administrative officers, including her vice presidents. She also said that what is required is that "people work together." ${ }^{187}$ Cooley, Klein, and Weeks maintained that delegation of responsibilities is an essential ingredient in successful operation of an institution. ${ }^{188}$ Adventist literature is not wanting in regard to promoting the idea of delegation of responsibilities. Maphosa believes that delegation motivates the people in the organization. ${ }^{189}$ Segovia stated that delegation is an essential component of leadership, for it can be the most important form of in-service training for future leaders. ${ }^{190}$ Brown recognized that presidents will not know everything, and will therefore gladly accept help and advice of his or her associates. ${ }^{191}$ The General

${ }^{185}$ In his inaugural address, installing Dr. JoAnn W. Haysbert as new president of Langston University, Dr. William R. Harvey, president of Hampton University, mentioned the complex roles a university president has to carry out in the 21 st century. Speaking on the importance of delegation of responsibilities, he said that leadership is the ability to inspire other people to conscientiously work together as a team in order to attain a common goal. William R. Harvey, inaugural address, September 30, 2006, www.hamptonu.edu/administration/president/speeches/haysbert_ianuguration/html (accessed 01-25-08).

${ }^{186}$ Harry Mayden, "Christian Administration: Relationships and Responsibilities," in Christ in the Classroom, (1997), 18:9.

${ }^{187}$ Lawrence, 166, 173.

${ }^{188}$ Cooley, Klein, and Weeks, in Lewis and Weems, Jr., 37.

${ }^{189}$ Maphosa, in Christ in the Classroom, (1994), 12:158.

${ }^{190}$ Segovia, in Christ in the Classroom, (1991), 2:231-233.

${ }^{191}$ Brown, 128. 
Conference Education Department of the SDA world church has underscored this broad concept of administration to be one of the characteristics of a successful Seventh-day Adventist college or university. ${ }^{192}$ The findings and literature, therefore, do warrant the need for intensifying presidents' attention to delegation of responsibilities and authorities to their support administrators for better operation.

Some other interesting findings can be noted in the overall performances and expectations of the general administrative functions as perceived by the status groups. Among the seven broad categories of functions, the general administration items became third in terms of the respondents' perception and expectation level. ${ }^{193}$ This shows that the presidents' general administrative responsibilities were considered more important than four other categories of functions as far as statistical findings are concerned. All 17 presidents were convinced that their actual and expected performances regarding preparation of board agendas could not be any better. All 18 board chairs felt that the presidents' adherence to educational philosophy should not be less than perfect, meaning they should maximize their efforts to the fullest of their abilities. ${ }^{194}$

\section{Finance-Related Functions-- Actual versus Expected}

Differences

The results reveal that there were significant statistical differences between

\footnotetext{
${ }^{192}$ See Appendix D, sec. 3.

${ }^{193}$ See Table 57 in Appendix M.

${ }^{194}$ See Table 10.
} 
perceived actual role performances and expected roles of the presidents for all of the following five items of finance-related responsibilities: ${ }^{195}$

\section{Participate in the preparation of annual budget of institution}

27. Ensure that the institution's budget, after approval by the board of trustees, is adhered to

28. Present institution's annual audited financial statements to board

29. Recommend to board the salary changes for faculty/staff positions

30. Accept accountability to board for financial operation of institution.

The above items of Null Hypothesis 3 were rejected. There were notable differences between how the presidents were actually involving themselves in the financial aspects and how they were expected to carry out these responsibilities. The respondents found that the presidents' actual perceived practices were lagging far behind the degrees of expectations by the same group. The findings definitively suggest that the presidents of the Seventh-day Adventist senior colleges and universities around the world should pay more attention to budget preparation, budget adherence, financial statement interpretation, faculty/staff salary change recommendations, and acceptance of financial accountability.

Item 26: The presidents' participation in the preparation of annual budget for the institutions was viewed as good performance when the survey was done. However, a higher expectation of this particular job was noted. ${ }^{196}$ The common view is that all chief

\footnotetext{
${ }^{195}$ See Table 29, sec. IV.

${ }^{196}$ See Table 29, sec. IV.
} 
executives may not have business education, training, and skill in financial details of administration, and therefore they do not actually have to prepare the school's operating budget by themselves but the expertise of the chief financial officers of the institutions could be used in doing this task. The president, as Ruger, Canary, and Land, and Smith believed, will bear ultimate responsibility for all major decisions, plans, and priorities imbedded in the budget, and therefore he or she must participate fully in the review and development of the budget. ${ }^{197}$ Brown at the Education Department of the SDA world church mentioned that estimating the budget needs of the institution is one of the duties of the president. ${ }^{198}$ The views of Ruger, Canary, and Land, and Smith, and Brown should be taken into consideration seriously to meet the expectations regarding the Adventist college presidents' involvement in the operating budget preparation for their institutions.

Item 27: This is about staying within the operating budget of the institution. Former President Gerhard Casper of Stanford University identified the annual budget control as the number one job of the university administration. ${ }^{199}$ The respondents of this study felt that the chief executives are "responsible for everything that happens within the institution" ${ }^{\prime 200}$ and therefore they should see that their schools adhere to the annual operating budget more than the perceived actual performance of this role the presidents

${ }^{197}$ Ruger, Canary, and Land, in Lewis and Weems, Jr, 96; see also Mark F. Smith, "Growing Expenses, Shrinking Resources," ACADEME: Bulletin of the American Association of University Professors (AAUP) 90, no. 4 (2004): 32-35.

${ }^{198}$ Brown, 65, E.

${ }^{199}$ The Stanford Daily Staff, "University Must Rethink the Role of President," www:stanforddaily.com/article/2001/2/9 (accessed January 22, 2008).

${ }^{200}$ Brown, 128. 
have demonstrated at the time the survey was done in 16 countries. It is noteworthy that this item, among all the 31 significant role items, showed the highest difference between the presidents' perceived actual and expected role performances. ${ }^{201}$ The findings also tend to indicate that there has been much agreement among the responding groups on the role expectations of this item than their largely variant perceptions of the actual shouldering of this responsibility. This could mean that in the Seventh-day Adventist higher education institutions throughout the world there may have been quite a few instances where budget has not been very realistic. The findings of this study suggest that a careful monitoring of a balanced budget implementation is very important for the presidents to look after.

Item 28: Presenting the institution's annual audited financial statements to board is an item that has the lowest but significant difference among all the 31 items having significant differences between the actual and expected roles. ${ }^{202}$ Perhaps this result could be attributed to the respondents' general observation that it is not the president but the chief financial officer who, on behalf of the president, presents and interprets to the board meeting the financial statements, prepared by the accounting staff and examined by the independent auditors. I agree with Ruger, Canary, and Land who argued that the presidents do not need to spend much time on the annual audit. The important task of the president, they said, is to understand the audit and other year-end financial statements, interpret the results to various constituencies, when needed, and address the problems

\footnotetext{
${ }^{201}$ See Table 59 in Appendix O.

${ }^{202}$ See Table 59 in Appendix $O$.
} 
revealed in the audit, audit process, and other year-end reports, ${ }^{203}$ with the help of the finance officers. Whatever may be the understanding of this role, the purpose would be to know whether the financial performances shown in the statement are likely to improve or deteriorate by year-end. The findings of this study corroborate to the fact that monitoring of financial performance follows from an effective reporting system.

Item 29: Recommending to the board of trustees the faculty's salary changes is another function of the presidents that showed a significant difference in perceptions between the actual and expected roles. The test result shows that the expectation for this particular job was higher than the perceived performance level of this role which was actually done in a moderate degree when this survey was done. While it is true that the Seventh-day Adventist organization all over the world has policies on compensations for all its employees that are reviewed periodically in light of the escalating economic needs of the society, it is possible that in some cases the college/university faculty's salaries are not equitable and their wages could be even lower than comparable faculty in other institutions. The findings of this study tend to suggest that on many SDA college and university campuses around the world the presidents should pay more attention to the compensations considering the employees' "preparation, experience, performances,"204 "effort, seniority, skills held, and job difficulty," ${ }^{205}$ which are often overlooked.

Item 30: This item is about accountability in financial matters. The results reveal

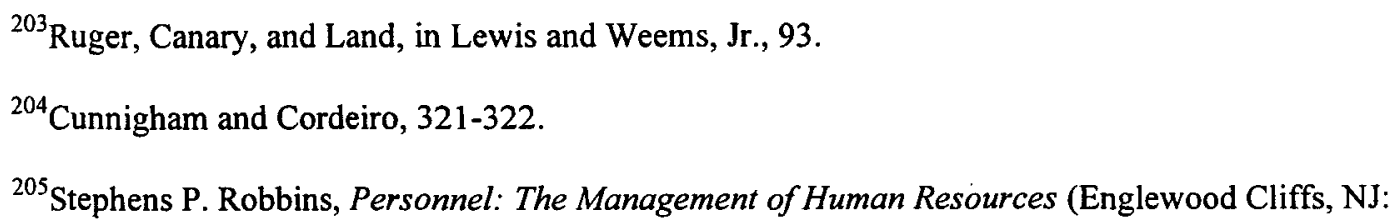
Prentice Hall, 1987), quoted in Narbarte, 104. 
that the respondents expected the presidents to perform significantly better than the perceived actual role performances in this area. This item occupies a third position as far as the rankings of the 31 significant items are concerned, ${ }^{206}$ indicating a far greater import on how the chief executives should handle fiscal stewardship. Expediently, the chief financial officers are often held responsible for any irregularities in financial matters. However, the findings of this study are in consonance with the literature ${ }^{207}$ that the administrators are ultimately accountable to the boards in greater measure for financial operation of the institutions. It is true, though, that the finance vice presidents and bookkeepers are there to help maintain accurate financial records. Nonetheless, the presidents are ultimately held responsible and should understand the basic accounting procedures and financial policies that are being observed. ${ }^{208}$ Like Ward, ${ }^{209}$ this study recognizes the presidents' acceptance of more accountability to the boards.

\section{Physical Facilities-Related Functions-- Actual versus Expected}

No differences

The findings of this study show that there were no significant statistical

$$
{ }^{206} \text { See Table } 59 \text { in Appendix } 0 .
$$

${ }^{207}$ Jacob E. Adams, Jr., and Michael W. Kirst, "New Demands and Concepts for Educational Accountability: Striving for Results in an Era of Excellence," in Handbook of Research on Educational Administration: A Project of the American Educational Research Association, ed. Joseph Murphy and Karen Seashore Louis, $2^{\text {nd }}$ ed. (San Francisco, CA: Jossey-Bass, 1999), 465.

${ }^{208}$ Cunningham and Cordeiro, 372-373.

${ }^{209}$ Lawrence, 431. During interview, David Ward, chancellor emeritus of the University of Wisconsin-Madison, told Lawrence that presidents' financial accountability has grown over the last 50 years. 
differences between the perceived actual role performances and expected role responsibilities of the SDA senior college and university presidents around the world for the following four of the six physical facilities-related functions:

31. Present to board the physical needs of the institution

32. Direct the preparation of plans for new buildings and installations

33. Plan for major repairs or alterations of plans, buildings and equipment

36. Ascertain that campus grounds are well kept through appropriate officer.

The above items of Null Hypothesis 3 were retained because there were no significant differences between how the respondents perceived the presidents performed and how they expected them to perform these roles. ${ }^{210}$ The presidents have done fairly well on 67 percent of the physical facilities-related functions. Their perceived actual performances were not significantly lower than what they were expected to perform.

Item 31: Physical needs of the institutions were presented to the boards at a satisfactory level. These needs include grounds, buildings, roads, furniture, equipment, supplies, transportation, parking, energy, utility, labs, communication services, central storage, warehouse spaces, gymnasium, athletic facilities, and such other physical facilities required to operate an institution. The General Conference Education Department of the Seventh-day Adventist world church has some suggested guidelines for physical facilities to establish, maintain, and operate SDA educational institutions. ${ }^{211}$ The findings suggest that this particular function is being carried out well to the degree of

\footnotetext{
${ }^{210}$ See Table 29, sec. $V$.

${ }^{211}$ Brown, 213-243.
} 
expectations of the institutions' board chairs and on-campus administrators. The presidents may have delegated much of the details of this responsibility to the finance vice presidents and on- and off-campus experts to look after the physical needs, and rightly so, for Ewing, Graves, and Landrebe stated that the president's primary duty cannot be focused on facilities, as there are other issues of leadership that demand greater attention. ${ }^{212}$ Adventist literature and job descriptions, which I have examined, do not say much about the SDA college and university presidents' physical facilities-related functions. However, at the World Education Advisory held at the General Conference headquarters, April 1996, the world education department directors voted a document that included proper and adequate physical facilities as one of the characteristics of a successful SDA college and university. ${ }^{213}$

Item 32: This is about embarking on plans for new buildings and installations for the institution. The presidents' actual performance of this particular role item was found to be close to the expectation mark of the responding groups. The findings suggest that the SDA senior college and university presidents in the 11 world divisions have engaged themselves to a satisfactory degree in directing the designated teams for drawing up new building and installation plans. The results also indicate that a slight increase in the attention to this function is expected. ${ }^{214}$ While saying that the presidents' attention cannot be focused primarily on buildings, Ewing, Graves, and Landrebe did not at the same time

\footnotetext{
${ }^{212}$ Ewing, Graves, and Landrebe, in Lewis and Weems, Jr., 116.

${ }^{213}$ See Appendix D, sec. 9.

${ }^{214}$ See Table 32, sec. V, \#32.
} 
totally exclude them from involvement in the facilities planning and maintenance activities. ${ }^{215}$ The SDA college presidents knew, I am sure, that new buildings and new installations of plants are important to provide needed facilities for new and upgraded academic programs and other services in order to attempt to achieve the mission of the institution, as President Shirley Ann Jackson at Rensselaer Polytechnic Institute, among others, realized and added a number of buildings and installations on her campus. ${ }^{216}$ The administrators of the Seventh-day Adventist institutions of higher education worldwide seem to believe what Winston Churchill once said: "We shape our buildings. Therefore, they shape us."217

Item 33: This had to do with major repairs or alterations of plants, buildings, and equipment. This particular job was done almost as expected. ${ }^{218}$ The results of this study could mean that the presidents realized that inadequate, defective, and non-functioning facilities could engender a sense of dissatisfaction and low morale among the faculty and students and so they have performed well. Ewing, Graves, and Landrebe took a strong position that whether facing severe facility problems or planning for major renovations or new construction, the president will have to take a leading role in planning and setting priorities. Fortunately, taking such a role does not mean that the president must have

${ }^{215}$ Ewing, Graves, and Landrebe, in Lewis and Weems, Jr., 116.

${ }^{216}$ Lawrence, 281. Francis Lawrence interviewed President Shirley Ann Jackson of Rensselaer Polytechnic Institute. She made substantial capital investments in building a number of new buildings, namely, residence halls, science complex, performing arts center, gymnasium, research facility, housing for graduate students and upper-class undergraduates. She also upgraded the outdoor athletic fields.

\footnotetext{
${ }^{217}$ Duderstadt, 151.

${ }^{218}$ See Table 58 in Appendix N.
} 
great knowledge about infrastructure, construction, or building codes, although some knowledge wouldn't hurt. ${ }^{219}$ President Duderstadt who served as president of University of Michigan took a course on modern architecture at Yale and worked for an architectureengineering firm in the 1960s, but he stayed away from any direct involvement in architectural issues as president. Instead, he relied on the chief financial officer and his experienced staff in the plant extension department. ${ }^{220}$ The findings of this study are supported by the above literature and practices mentioned in this section.

Item 36: The presidents' actual perceived performances of ascertaining that the campus grounds are well-kept were found to be not significantly different from the respondents' role expectations of this item. The findings indicate that the presidents' attention to this particular area is satisfactory. In all the SDA campuses the presidents seemed to have exhibited an aesthetic sense keeping the school grounds well. My visits to many SDA colleges and universities and the pictures I have seen in the SDA magazines, convince me that the SDA campuses with good landscaping and proper maintenance of grounds do indeed look beautiful. The physical appearances of the SDA campuses attract large numbers of students and visitors. Public and private institutions of higher education are also equally serious in maintaining well-kept school plants. Among others, Kerwin who served as president at the American University in Washington D.C. in the 1990 s, made notable facilities improvement of the campus. ${ }^{221}$

${ }^{219}$ Ewing, 117.

${ }^{220}$ Duderstadt, 154.

${ }^{221}$ American University, "Cornelius M. Kerwin: Biography," www1.american.edu/president/bio/index.html (accessed May 8, 2009). 


\section{Differences}

The remaining two items of the physical facilities-related functions showed significant statistical differences between the perceived actual role performances and role expectations of the SDA college and university presidents as the following present:

\section{Recommend to board for equipment acquisitions}

35. Ensure that physical plant master plans are current and followed closely.

The above items of Null Hypothesis 3 were rejected. Significant differences in perceptions were found to exist between the actual role performances and expected role responsibilities of the presidents of the SDA senior colleges and universities around the world. ${ }^{222}$ The results reveal that only 33 percent of the physical facilities-related functions had significant differences between the actual and expected roles.

Item 34: The presidents' attention to working with the boards in acquiring equipment was not on a par with the expectations of the board chairs and on-campus administrators who responded to this question. Equipment acquisition is important since the existing equipment might be aging fast and needs replacement. In the winter of 2008 President Bob Knight of Clark College and in January of 2007 President Graham Spanier of the Pennsylvania State University, among others, realized this fact and embarked upon projects to acquire necessary equipment for educational needs of their institutions. ${ }^{223}$ Brown at the General Conference Education Department of the Seventh-day Adventist

${ }^{222}$ See Table 29, sec. V.

${ }^{223}$ Clark College, "College President's Role in Equipment Acquisition," www.clarkcollegefoundation.org/newsletter.php?id=15 (accessed July 7, 2009). See also Graham B. Spanier, president of The Pennsylvania State University, "2007 State of the University Address," 4-5, 7, www.president.psu.edu/sou/pdf/sou07_transcript.pdf (accessed July 7, 2009). 
world church included this item in his volume as the college/university president's job. ${ }^{224}$ Somehow throughout the world this particular role performance did not meet the expectations. The findings of this study could mean that either it was felt there were not enough equipment needs at the schools or the chief executives were not known to have presented these needs to the boards. However, the results reveal that the respondents expected the presidents to pay more attention to this activity than their actual performance level. Adequate equipment can help augment the effectiveness in the overall performances of the administration.

Item 35: This is about the physical plant master plans. The presidents' perceived actual performances worldwide differed significantly from the respondents' expectations of this role. The presidents' performances lagged behind the expected mark. The findings of this study behoove that the presidents recommend appointing master planning committees that will provide guidance and oversight to the master planning process. Like Harvard's Allston Campus administrators, the SDA respondents seemed to realize that the master plan is necessary because it "describes an institution's existing facilities, longrange planning goals, and proposed projects." It should be periodically updated, renewed, and amended as deem necessary. ${ }^{225}$ In 1997, the World Education Advisory ${ }^{226}$ at the General Conference Education Department of the SDA world church came up with the idea of the college plant development that would help provide an ideal physical

\footnotetext{
${ }^{224}$ Brown, 65, G.

${ }^{225}$ Harvard University Allston Campus, “University President's Role in Master Planning," www.allston.harvard.edu/imp/imp.html (accessed July 7, 2009).

${ }^{226}$ See Appendix D, \#9.
} 
environment for nurturing the work and scholarship that goes on the campus. Like many colleges and universities that we can look up in their websites, the University of

Washington has developed a new master plan. They review it and keep it current. ${ }^{227}$

Likewise, the board chairs and on-campus administrators of the SDA senior colleges and universities worldwide expect the presidents to keep their physical plant master plans current and follow them.

\section{Community- and Alumni-Related Functions-- Actual versus Expected}

\section{Differences}

This study shows that significant statistical differences exist between the perceived actual role performances and role expectations of the presidents for all of the following six items of community- and alumni-related functions:

\section{Assist in raising funds for support of institution}

38. Represent institution to churches of the community

39. Present reports about institution to community and alumni

40. Represent institution as its official spokesperson to public

41. Associate closely with alumni groups

42. Listen to the constituency and alumni.

The above items of Null Hypothesis 3 were rejected. The presidents' handling of these roles was expected to be much more extensive than how they were perceived

\footnotetext{
${ }^{227}$ University of Washington, "University President's Role in Master Planning," www.washington.edu/community/cmp_site/Plan_Background.html; see also "University of Washington Master Plan," www.washington.edu/community/files/Semi-AnnualReport-01-07.pdf (accessed July 7, 2009).
} 
carrying out in their actual practices. The presidents were lagging far behind the expectation levels of the board chairs and the vice presidents. The findings suggest very strongly that the SDA senior college and university chief executive officers around the world should step up their efforts to maximize the performances of the community- and alumni-relations activities.

Item 37: The results show that at the time of this survey the presidents of the SDA senior colleges and universities around the world were not doing the fundraising work according to the expectations. This function has the highest difference between the perceptions of the actual role and expected role among all the 31 items with significant statistical differences. ${ }^{228}$ The findings suggest that the presidents need to engage themselves in much more fundraising activities for their institutions than what they were found doing. Basinger, Calian, and Leavitt concurred that the presidents should allocate more time involving themselves in securing friends, funds, and connections necessary to undergird the mission of the institutions. ${ }^{229}$ On the contrary, Hesburgh accused the college presidents of spending too much time raising money. ${ }^{230} \mathrm{I}$ think the ideal length of time the presidents, especially at the SDA colleges and universities, may need to spend for raising funds for their institutions could be best determined on a case-by-case basis.

Item 38: This item is about the presidents representing the SDA colleges and universities to the churches of the constituencies. Apparently, they need to pay more

${ }^{228}$ See Table 59 in Appendix 0.

${ }^{229}$ Rebekah Burch Basinger, C. Samuel Calian, and Robert F. Leavitt, "The President's Role in Institutional Advancement," in Lewis and Weems, Jr., 127.

${ }^{230}$ Paolitto, 2. 
attention to this particular activity. Since the SDA colleges and universities are religious institutions, they depend on the churches for their support in terms of funds, students, and publicity. I think Basinger, Calian, and Leavitt ${ }^{231}$ and Cushing, Morgan, and Aymer ${ }^{232}$ are right when they said that the chief executive officers need to spend significant time each year with the church officials to acquaint them with the various aspects of the institutions so that they will be informed of the needs, challenges, opportunities, issues, and trends and respond to them accordingly. Although the Seventh-day Adventist senior college and university presidents were found doing good in this area, all five status groups participated in this study expect them to do better. ${ }^{233}$

Item 39: The presidents' performances of presenting the reports about their institutions to the community and alumni lagged behind the expectation mark. The results show that this job was not done in mediocrity. However, the respondents expected far better performances. Charles Vest, who served as president of the Massachusetts Institute of Technology from 1990 until 2004, said in his interview with Francis Lawrence, then president of the State University of New Jersey, that his community relations helped do so much good for MIT. ${ }^{234}$ In some cases the presidents may have great challenges to present the reports to the communities about their colleges and universities, which are known as religious institutions. But the chief executives, the

\footnotetext{
${ }^{231}$ Basinger, Calian, and Leavitt, in Lewis and Weems, Jr., 161.

${ }^{232}$ Cushing, Morgan, and Aymer, in Lewis and Weems, Jr., 155.

${ }^{233}$ See Table 32, sec. VI, 38.

${ }^{234}$ Lawrence, 394.
} 
public relations departments, and the alumni offices of the SDA colleges and universities can work together and develop creative ideas to raise friends in the communities so that they will feel a sense of ownership of the institutions and support them.

Item 40: This item had to do with representing the institutions to the public. The presidents are the official spokespersons for their institutions. According to the findings of this study, they should focus more on this activity than what they were perceived doing at the time of this survey. There may have been a time when the SDA education institutions were insulated from the public. But Christian educators, such as Basinger, Calian, and Leavitt, feel that those days are long past. Today, the presidents are visible to the public and frequently speak for their institutions and by words they build confidence among key people in the gift-worthiness of their schools. ${ }^{235}$ Like the findings of Bagin and Gallagher, ${ }^{236}$ this study shows that it is very important that the presidents of the SDA colleges and universities make use of any proper opportunities they can find to represent their institutions as "chief persuaders"237 to the public for their support.

Items 41 and 42: These two items had to do with the presidents working with and listening to the constituency and alumni groups. The constituency for an SDA college or university encompasses the churches and patrons within its jurisdiction. The alumni could extend beyond the college's geography jurisdiction. It is important for a college and university president to have close ties with these two entities. Both of these roles

${ }^{235}$ Basinger, Calian, and Leavitt, in Lewis and Weems, Jr., 128-129.

${ }^{236}$ Don Bagin and Donald R. Gallagher, The School and Community Relations (Needham Heights, MA: Allyn \& Bacon, 2001), 117, quoted in Nudd, 9.

${ }^{237}$ Murphy, 49. 
showed significant differences between the actual and expected roles. The presidents were expected to do more in these areas than what they were perceived doing at the time of this survey. There are many instances of constituency and alumni providing financial resources, expertise, and other support to higher education institutions in the United States and abroad. They will continue doing so with greater commitments, if the presidents would keep their listening communication open.

While it is true that most of the Seventh-day Adventist colleges and universities around the world do not accept government funding for operating, a large number of students attending SDA institutions of higher education in certain countries may receive federal and state grants and loans that go directly into their student accounts to help toward education costs. ${ }^{238}$ Other sources of funds are "endowed money," "scholarships," "and contributions from local churches" that assist in students' financial needs while in college. Research grants and donations from alumni and donors are generally designated for specific educational projects. The major chunk of financial assistance and subsidies comes from the church that operates the institutions. The appropriations from the church's higher bodies, students' tuition income, and profits from school industries, if any, are not enough to meet the institutions' operating expenses. According to Nudd, funding from donors is very important to run the institutions. ${ }^{239}$ Today's college

\footnotetext{
${ }^{238}$ Gene Edelbach, “Adventist Education: Mapping Your Future," Adventist Review, online edition, www.adventistreview.org/9944/story2.html (accessed December 28, 2009). In some third-world countries I know the public funding can become conditional to government interference in the governance of the colleges and universities. Some SDA educational institutions were lost to the government because of accepting public funding. Generally, the students at the private institutions in many of these countries do not receive government grants and loans for their education.

${ }^{239}$ Nudd, 9 .
} 
presidency, Rile reported, has become primarily concerned with fundraising ${ }^{240}$ and the president is key to public relations that serves as a vehicle to do this work.

The findings also suggest that the presidents need to build a systematic communication network to maintain cordial relations with the public, community, constituency, churches, and alumni. David Hubbard, former president of Fuller Theological Seminary, strongly proposed the idea of communicating to the stakeholders. ${ }^{241}$ It is only when the public feels the ownership that they will do their very best in supporting the institutions by providing money and other services, when requested by the college or university administration. Birkenstock, who served as president at an international Adventist university overseas, recognized that there will always be a shortage of finances and therefore donors play a very important role. ${ }^{242}$ Dove, Lindauer, and Madvig, ${ }^{243}$ Rosso and Tempel, ${ }^{244}$ and Zimmerman and Lehman ${ }^{245}$ felt the need of maintaining stronger ties with the stakeholders. They described a wide variety of methods that the not-for-profit organizations use to raise money for the institutional advancement centered around the mission. This study also found that the public and

${ }^{240}$ Rile, 5.

${ }^{241}$ Cushing, Morgan, and Aymer, in Lewis and Weems, Jr., 157.

${ }^{242}$ Birkenstock, 46. Birkenstock served as president at Adventist International Institute of Advanced Studies, a graduate school in the Philippines, operated by the General Conference of Seventh-day Adventist world church. See also Basinger, Calian, and Leavitt, in Lewis and Weems, Jr., 137.

${ }^{243}$ Kent E. Dove, Jeffry A. Lindauer, and Carolyn P. Madvig, Conducting a Successful Annual Giving Program (San Francisco, CA: Jossey-Bass, 2001).

${ }^{244}$ Henry A. Rosso and Eugene R. Tempel, Achieving Excellence in Fund Raising, $2^{\text {nd }}$ ed. (San Francisco, CA: Jossey-Bass, 2003).

${ }^{245}$ Robert M. Zimmerman and Ann W. Lehman, Boards That Love Fundraising: A How-to-Guide for Your Board (San Francisco, CA: Jossey-Bass, 2004). 
alumni activities ranked first both for the actual role performances demonstrated by the Seventh-day Adventist college/university presidents worldwide and the responsibilities expected of them to perform in this category of functions (Table 57 in Appendix M).

\section{Spiritual Life-Related Functions-- Actual versus Expected}

No difference

The results reveal that there was no difference between the perceived actual role performance and role expectation of the presidents for the following one item of the spiritual life-related functions:

\section{Focus on Christian philosophy of institution.}

The above item of Null Hypothesis 3 was retained. There was no significant statistical difference found between how the respondents perceived the presidents were actually performing and how they expected them to perform this role. The findings definitively suggest that the presidents met the expectation level of the board chairs, presidents, academic vice presidents, student service vice presidents, and finance vice presidents for this item. This is not surprising because all the college and university presidents are Seventh-day Adventist Christians. They are fully aware of the essential characteristics of Seventh-day Adventist education, such as "identity, mission"246 and philosophy. ${ }^{247}$ They were fully committed to the mission and philosophy of their institutions and try their very best to reflect these characteristics in all their practices.

\footnotetext{
${ }^{246}$ Geraty, 5 .

${ }^{247}$ Lashley, 7.
} 


\section{Differences}

The remaining four spiritual life-related items showed significant statistical differences between the perceived actual role performances and expected role responsibilities of the presidents as the following present:

43. Assume primary responsibility for promotion of students' religious life at the institution via delegation

44. Assume primary responsibility for promotion of religious life of faculty and staff at the institution via delegation

45. Take ultimate responsibility for planning midweek chapels, vespers, weeks of spiritual emphasis and other religious programs on campus via delegation for spiritual nurture

46. Manifest Christ-like character in spiritual leadership roles.

The above items of Null Hypothesis 3 were rejected. The results reveal that there were significant differences between the extent of perceived actual performances of these roles and the degrees of these expected role responsibilities of the SDA senior college and university presidents around the world as viewed by the respondents. The groups' expectations regarding these presidential activities were significantly higher than the perceived actual performances of the same roles. The findings suggest that the presidents were not doing as much as they should in these spiritual responsibilities. Table 57 in Appendix M shows that the spiritual life-related responsibilities received second position among all the seven broad categories of functions as far as the presidents' actual performances and role expectations as viewed by the responding groups are concerned. 
These findings attach importance to the spiritual dimension of the SDA college and university presidency.

Items 43, 44, and 45: These items had to do with promoting religious life of the students, faculty, and staff through many activities. The SDA college and university presidents should pay attention to their religious duties more than what they were found involving themselves in. According to King, the Adventist education "must have a spiritual dimension. It must speak to the most vital area of life, namely, humanity's need for a relationship with a transcendent God."248 The presidents need to assume more primary responsibilities in seeing that various religious programs, such as chapels, and vespers, are adequately carried out on their campuses to help promote and nurture the religious life of faculty, staff, and students. In many overseas institutions that I know of from my personal on-site visitations, the college/university president is considered as head of all religious activities. Akers stated that the president is the "high priest" of the campus. ${ }^{249}$ Although in all campuses there are college/university chaplains or someone vested with the responsibility of religious affairs, the president is ultimately responsible for the spiritual tone of the campus. Kamwendo ${ }^{250}$ stated that the chief executives of the SDA colleges and universities are held responsible for everything that happens on the campuses, including spiritual activities, such as "worships, chapels" and other religious

${ }^{248}$ Greg A. King, "Should Adventist Colleges Require Religion Classes?" The Journal of Adventist Education 68, no. 3 (2006): 22.

${ }^{249}$ George Akers made this comment while participating in the preliminary questionnaire study. His written comment is in my personal file Akers served as academic vice president at Washington Adventist University, Maryland, and director of education for the Seventh-day Adventist world church.

${ }^{250}$ Kamwendo, in Christ in the Classroom, ((2001), 28:142. 
programs in the "spiritual realm." The religious objective is considered to be the most important dimension of Seventh-day Adventist education.

Item 46: The presidents' spiritual leadership is another item that did not meet the expectations of the status groups. A better performance was expected of the presidents. Manifesting Christ-like character in spiritual leadership role means demonstrating servant leadership qualities. According to Schantz, servanthood is not servitude. Servitude is demeaning for it is a status forced on people, depriving them of the freedom of choice. Servanthood is a voluntary action, choosing to be of service to others. ${ }^{251}$ The findings of this study suggest that the presidents need to work hard to "exemplify more of Jesus' leadership style of organizing, training, and meeting the needs of the people."252

\section{Final Comments on No Differences and Differences Between Actual and Expected Roles}

No differences

Sixteen out of 47 items were not found to be statistically significant between the presidents' actual role performances and expected roles. There could be several reasons why there were no significant differences between how the presidents were perceived to have actually performed and how they were expected to perform.

The first and the foremost reason, I think, was that the parameters of the procedures, guidelines, policies, and protocols of the above functions were clear enough

${ }^{251}$ Iris S. Schantz, "Leadership and Servanthood: Challenges, Tensions and Possibilities in Christian Organizations," in Christ in the Classroom, (1993), 7:243-245. Schantz of Newbold College, Berkshire, England, unequivocally stated that Christian leadership is servant leadership.

${ }^{252}$ Ibid. 
to the board chairs, presidents, and vice presidents.

Second, the boards of trustees must have done a good job in the shared governance of the colleges and universities harmonious to the governing rules.

Last, the Education Departments at the union conferences, divisions, and the General Conference under the jurisdictions of which the institutions belong, have served well as watchdogs, ensuring that the mission of the campuses was not lost sight of.

\section{Differences}

The fact that the SDA senior colleges and universities included in this study are located on six continents, having a wide variety of demographics, societal complexities, economic disparities, political conditions, cultural diversities, and varied perspectives may have been the cause for the differences of perceptions between the actual performances and expected roles.

The literature points out that the role and power of president, rector, chancellor, or vice chancellor vary greatly from country to country and even from institution to institution. In general, presidents, chancellors, and vice chancellors in North America enjoy more autonomy than those in other parts of the world-in part, perhaps, because, unlike those in many industrialized countries, their universities are not wholly dependent upon the state for both financial support and direction. The presence of large numbers of independent universities in the United States makes the role of the presidents distinct from the university presidents in other countries. ${ }^{253}$

\footnotetext{
${ }^{253}$ Rhodes, quoted in Werner Z. Hirsh and Luc E. Weber, 12.
} 


\section{Conclusions}

The following conclusions are in alignment with the five research questions and the three hypotheses of this study:

1. The presidents of the Seventh-day Adventist senior colleges and universities around the world carried out 47 responsibilities in various levels of perceived performances. More than two-thirds of all the roles were performed at above the moderate level. All of the community- and alumni-related activities were perceived to have been conducted at the strong performance level. The guiding force behind the overall good performances, I would say, is the theme of the Seventh-day Adventist philosophy that has pervaded very strongly in all areas of the actual functions of the presidents.

2. The presidents of the SDA senior colleges and universities around the world were expected to perform 47 responsibilities in various levels of expectations. About three-fourths of all their duties were expected to be performed at above the moderate level. All of the community- and alumni-related functions and more than two-thirds of the general administrative functions, up by 56 percent over the actual performances, need the highest level of performances. Again, the SDA philosophy is the most prominent underlying principle in all the expected role responsibilities of the presidents.

3. All five status groups participated in this study are cohesive. There is an interuniversity and intra-university consensus among the off-campus board chairs, on-campus presidents, academic vice presidents, student service vice presidents, and finance vice presidents of the Seventh-day Adventist institutions of higher education around the world. 
There is agreement in perceptions between them on the presidents' perceived performances of all 47 actual roles.

4. There is harmony among the off-campus board chairs, on-campus presidents, academic vice presidents, student service vice presidents, and finance vice presidents of the Seventh-day Adventist senior colleges and universities worldwide who participated in this study. The inter-university and intra-university cohesiveness demonstrated by these five status groups confirms the SDA senior college and university presidents' responsibilities they are expected to perform in all 47 expected roles.

5. Two thirds of all the presidents' actual versus expected roles examined in this study need a greater attention by the presidents for their improved performances. The most important area is finances. The presidents are expected to see that their actual performance level reaches the higher expectation level in matters of operating their institutions within the board-approved budget.

\section{Recommendations}

\section{For Practice}

Based on this study the following recommendations were made for practice in the Seventh-day Adventist higher education:

1. Areas of president's functions recommended for first top priority attention:

Follow operating budget, assist in raising funds, and be accountable for financial operation.

2. Area of president's functions recommended for second top priority attention:

Be responsible for religious life on campus. 
3. Areas of president's functions recommended for third priority attention:

Have good relationships with the churches, community, constituency and alumni and give them reports periodically about the ins titution

Take leading roles in delegating responsibilities, authorities, and needed resources.

The concept of delegation is viewed differently in different parts of

the world. While it is ideal for the chief executives to delegate certain responsibilities, authorities, and needed resources to their vice presidents and other administrators, it may be expected that in some settings the presidents get themselves more directly involved in those functions. This study recommends that the demographic conditions and cultural expectations of the campuses need to be considered in matters of the extent of delegation of duties.

Review organizational chart, adhere to educational philosophy, address grievances, and foster faculty research

Guard faculty's academic freedom and review accrediting agency's reports, and

Recommend to board for faculty's position and salary changes.

4. Areas of president's functions recommended for greater attention:

Manifest Christ-like servant leadership and be responsible for religious life on campus.

5. Areas of president's functions that need attention:

Dialogue frequently with students

Take ultimate responsibility in hiring, promoting, demoting, and dismissing employees, and 


\section{Present institution's annual audited financial statements to board.}

6. Review the General Conference Education Department's 12 characteristics of a successful Seventh-day Adventist College or University (see Appendix D of this study) to attempt to improve on the role expectation areas where inadequacies were found.

7. Review Walter Brown's list of major functions of the Seventh-day Adventist college/university presidents included in chapter 2 of this study to attempt to improve on the areas where the president's role performances were not found to be as expected.

8. The Education Department of the General Conference of Seventh-day Adventist world church, in collaboration with the Accrediting Association of Seventh-day Adventist Schools, Colleges, and Universities (AAA), Division and Union Conference/Mission Education Departments, and college/university administrations develop a model of college/university president's job description.

\section{For Future Research}

The following recommendations are presented for future research, based on the results obtained from this study:

1. This dissertation sought the perceptions of the university and senior college administrators on the actual and expected roles of the presidents of the Seventh-day Adventist senior colleges and universities in all the geographic and administrative divisions of the Seventh-day Adventist world church. It will be interesting to discover the perceptions of the faculty, staff, students, board members, and parents on these issues in selected SDA colleges and universities. 
2. A separate, new dimension of presidents' functions could be incorporated to do a more in-depth study on their relationships to the boards of trustees, determining their actual and expected performances on the SDA campuses of higher education.

3. A study could be conducted to survey the perceptions of similar administrators in this study as to what could account for the wide disparities between their perceptions of certain actual and expected roles.

4. Could the roles of the university and senior college presidents be more streamlined? A study could be conducted among selected SDA institutions of higher education to determine whether the roles that scored at a low level for both actual and expected functions should be revisited for possible elimination.

5. To ascertain greater participation, proper understanding, and accurate response to the questionnaire items, it is recommended that the instrument be translated into local languages for future research of this nature. 
APPENDIX A

RESEARCH PROTOCOL 


\section{RESEARCH PROTOCOL}

Researcher: Sunith K. Das, Ph.D. Candidate, Department of Educational Administration and Leadership, 4775 Kimber Lane, Berrien Springs, Michigan 49103, U.S.A. Phone: (616) 473-1612 (Home), (616) 471-6149 (Office). Fax: (616) 471-3119. E-mail: <dass@andrews.edu>

Research Advisor: Dr. Lyndon G. Furst, Dissertation Committee Chair, and Professor, Department of Educational Administration and Leadership, Andrews University, Berrien Springs, Michigan 49104, U.S.A. Phone: (616) 471-6701. E-mail: <furst@andrews.edu>

Title of Research: A Study of the Role Expectations of the Presidents of the Selected Seventh-day Adventist Universities and Senior Colleges as Perceived by Five Status Groups.

Purpose of Study: To investigate concerning degrees of common agreement and disagreement on actual and expected role performances of the presidents of the selected Seventh-day Adventist universities and senior colleges as perceived by the status groups.

Methods and Procedures: This is a quantitative study. An anonymous survey has been designed to solicit anonymous responses from selected Seventh-day Adventist educational administrative officers, also called status groups. An approval letter will be sent out to all the participants of the research from the General Conference Department of Education or from the School of Education, Andrews University. This will be followed by the researcher's explanation and instruction letter, ratified by the research advisor. Next, a questionnaire will be mailed to the subjects and anonymous data will be collected by mail from them. There are 47 close-end questions and 7 open-end questions distributed in the questionnaires among 7 dimensions of presidential functions. Each dimension has statements of presidential functions arranged on a scale from 1 to 5 . 1 means the subject strongly disagrees and 5 means the subject strongly agrees. The subject will circle one number for each statement in both the grids, titled, Actual Role Performance and Expected Role Responsibility. The questionnaire, written in minimally technical terms and which appropriately provides information, will take about 20 minutes to complete. The original copy of the completed questionnaire duly filled out by the participants, will be returned to the researcher in an enclosed self-addressed, postage-stamped envelope. The dissertation proposal, earlier approved by the dissertation committee and dated June 1, 2000, and June 2, 2000, has three null hypotheses. Hypotheses 1 and 2 will be tested by one-way ANOVA, while Hypothesis 3 will be tested by $t$-tests.

Time Frame of the Research: The research subjects is scheduled to begin in November 2001 and end in April 2002. The questionnaires will be mailed to the participants in December 2001. The initial data collection is expected to be completed by February 2002. March 2002 and April 2002 have been set aside for data processing. 
Subjects: The population for this study includes 140 adult competent subjects, such as 28 presidents, 28 vice-presidents for academic administration, 28 vice-presidents for student services, 28 vice-presidents for finance, and 28 board chairs, selected from a total of 28 Seventh-day Adventist universities and colleges in 16 countries offering graduate programs. The criteria for the inclusion of the subjects are based on their job positions that classify them as administrative team. The selection of the participating institutions is based upon the classification listed in the Seventh-day Adventist Yearbook 2001. The subjects will be involved anonymously as they respond to the questionnaire to be sent to them the mail.

Benefits/Results: The subjects will not receive any direct benefits from participating in the study. However, the results may help the college/university president in his/her professional role performance. Also, the information collected during this study will be included in a doctoral dissertation, and may be presented or published in professional meetings or journals to further expand the understanding of the relationship between president's role performances and his/her role expectations and also to avoid conflicts.

Risks: There are no risks, discomforts, stress, or invasion of privacy during or as a result of the subjects' participation in the study. This research does not place the subjects at risk of criminal or civil liability, or cause damage to the subjects' financial standing, employability or reputation.

Voluntary Participation: The subjects have the right to refuse to participate or to discontinue to participate at any time after research involvement begins without experiencing negative experiences, penalties or prejudice or denial of benefits to which the subjects are otherwise entitled.

Confidentiality: Each set of the questionnaires is color coded to identify the five status groups by their job titles, not by their names, for the purpose of the research. When the sealed envelopes containing the completed questionnaires are returned, the sending institutions are acknowledged for the purpose of follow-up, if needed. The subjects will not write their names or their institutions' names on the questionnaires. Once the envelopes are unsealed, separated from the questionnaires, and the completed forms are mixed with the other questionnaires, and their data are entered, there is no way of linking any subject to any information received. The procedures ensure the highest level of anonymity and confidentiality possible.

Waiving of Signed Consent Documentation: This study qualifies for a waiver of the signed consent documentation from the subjects, because of the following reasons:

1. The research will use questionnaires which will be mailed individually to the subjects under their job titles and returned anonymously to the researcher duly completed. The information will be recorded in such a way that the subjects cannot be identified directly or through identifiers linked to the subjects. 
2. The only record linking the subject and the research would be the written signed consent document and the principal risk would be potential harm resulting from a breach of confidentiality.

3. The research presents no more than minimal risk of harm to subjects and involves no procedures for which written consent is normally required outside of the research context.

4. The waiver will not adversely affect the rights and welfare of the subjects.

5. Since the questionnaire will be sent directly to the subjects at various educational institutions within and outside of America, the research could not practicably be carried out without the waiver of signed written informed consent form.

6. Whenever appropriate, the subjects will be provided with additional pertinent information during and after participation.

7. The cover letter explaining the purposes and procedures of the research may substitute for the signed consent form. It avoids any implication that there is a release of liability for negligence or a waiver of the subject's legal rights.

8. The anonymous return of the questionnaire serves as a form of implied consent.

9. The subjects have the opportunity to ask questions and receive satisfactory answers from the researcher or research advisor before and while participating in the study.

10. The subjects can contact an impartial third party, namely, Dr. Hinsdale Bernard, Program Coordinator, Educational Administration and Leadership, Andrews University, Berrien Springs, Michigan 49104, U.S:A., regarding any complaint that they may have about the study. He can also be reached by phone, fax, and e-mail as follows: Phone: (616) 4716702. Fax: (616) 471-6374. E-mail: <hbernard@andrews.edu>

Ethical Responsibilities: The researcher is fully aware of his ethical responsibilities to the subjects and pledges to do his best to safeguard all the accepted norms during the duration of research.

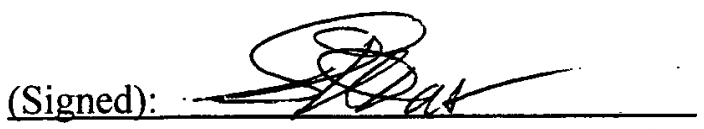

Dated: $10-10-01$

Sunith K. Das, Researcher/Ph.D. Candidate

Screened by Dr. Lyndon G. Furst, Research Advisor and Dissertation Committee Chair, and Professor of Educational Administration and Supervision, School of Education, Andrews University. 
APPENDIX B

ENDORSEMENT OF THE STUDY 

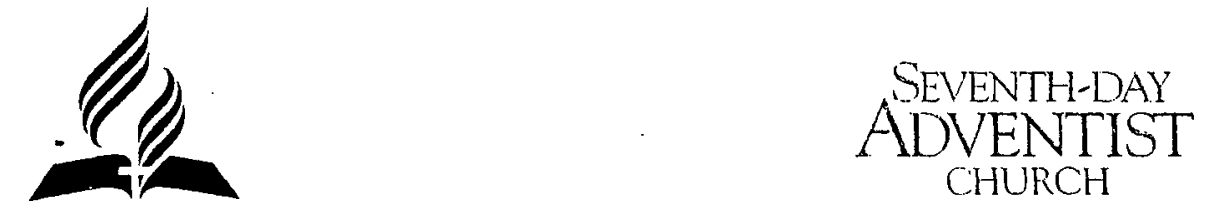

General Conference

World Headquarters

Departinent of Education

To:

Board Chairs and Administrators of Adventist

Colleges and Universities

12501 Old Columbio Pike

Silver Sping, Monland

20904-6600 USA

Telephone: (301) 68060000

From:

C. Garland Dulan

Fox: (301) 622-9627

Subject: $\quad$ Research by Sunith Das

Date: $\quad$ September 10, 2004

- Dear Leaders:

Mr. Sunith Das, a doctoral student in the school of Education at Andrews University, is undertaking a research project on the role of Adventist college and university presidents/rectors and the expectations of administrators who relate to them.

The General Conference Department of Education has endorsed Mr. Das' initiative and looks forward to receiving from him the results of this international survey, which we believe will strengthen Adventist education.

We will appreciate it if you would facilitate this important project by filling out the enclosed questionnaire and returning it promptly to Mr. Das.

With all best wishes and thanks,

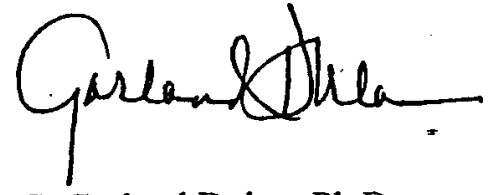

C. Garland Dulan, Ph.D.

Director 

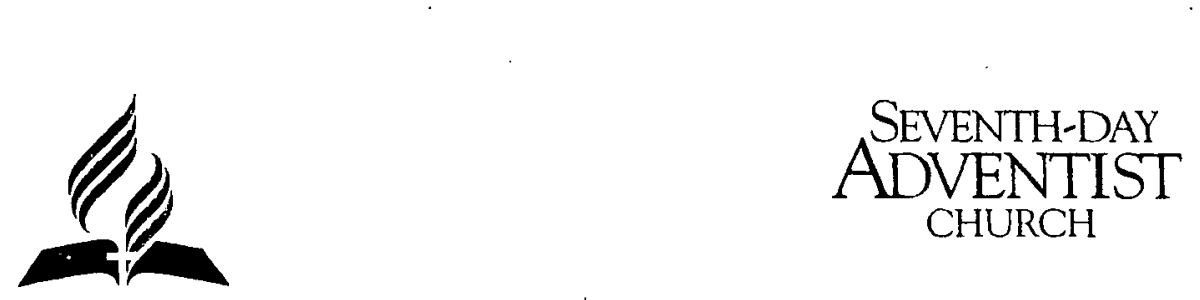

To:

Board Chairs and Administrators of Adventist Colleges and Universities

Departmenl of Education

12501 Old Columbia Pike Silver Spring, Marylond 20904.6600 USA Telephone (301) 680-6000

From: Humberto M. Rasi Fox (301) 622.9627

-Subject: $\quad$ RESEARCH BY SUNITH DAS

Date: - December 20, 2001

Dear leaders:

Mr. Sunith Das, a doctoral student at the School of Education of Andrews University, is undertaking a research project on the role of Adventist college and university presidents/rectors and the expectations of administrators who relate to them.

The General Conference Department of Education has endorsed Mr. Das' initiative and looks forward to receiving from him the results of this international survey, which will strengthen Adventist education.

We will appreciate it if you would facilitate this important project by filling out the enclosed questionnaire and returning it promptly to Mr. Das.

With Christian greetings and best wishes for the New Year, your brother,

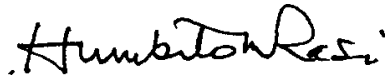

Humberto M. Rasi

Director 
APPENDIX C

SAMPLE INFORMED CONSENT DOCUMENT 


\section{SAMPLE INFORMED CONSENT DOCUMENT}

Researcher: Sunith K. Das, Ph.D. Candidate for Educational Administration \& Supervision, School of Education, Andrews University, Berrien Springs, Michigan 49104, U.S.A. Phone: (269) 473-1612 (H). Fax: (269) 471-3119. E-mail: <dass@andrews.edu>

Research Advisor/Dissertation Committee Chair: Hinsdale Bernard, Ph.D., Professor, Educational Administration and Leadership, School of Education, Andrews University, Berrien Springs, Michigan 49104, U.S.A. Phone: (269) 471-6702. Fax: (269) 4716374. E-mail: <hbernard@andrews.edu>

Title of Research: Role Expectations of Presidents of Seventh-day Adventist Senior Colleges and Universities as Perceived by Status Groups.

Purpose of Study: I have been told that the primary reason for conducting this research is to investigate degrees of common agreement and disagreement on actual and expected role performances of presidents of selected Seventh-day Adventist universities and senior colleges, as perceived by the status groups.

Methods and Procedures: I have been told that a survey has been designed to solicit responses from the selected Adventist educational administrative officers, including me. The population for this study includes a total of 140 subjects, distributed equally among five status groups, such as, (1) college/university board chairs, (2) college/university presidents, (3) vice presidents for academic administration, (4) vice presidents for student services, and (5) vice presidents for finance. These subjects have been selected from the 28 SDA universities and senior colleges in 17 countries that offer graduate degree programs. Data will be collected anonymously by mail from these 140 subjects on 47 close-end questions and 7 optional openend questions distributed in the questionnaire among 7 dimensions of presidential functions.

I am told that each participant was mailed an approval letter from the General Conference's Education Department followed by a request letter and a simple instruction from the researcher. Further, I am told that the questionnaire, written in non-technical terms and designed in user-friendly format, will not take more than 20 minutes to complete.

I am told that participant within the U.S.A. will use the self-addressed, postage stamped envelope, while participant outside of the U.S.A. territories will please affix his/her country's' required postage stamps on the self-addressed envelope provided herein by the researcher. The original copy of the completed questionnaire will be mailed to Sunith K. Das, $4775 \mathrm{Kimber}$ Lane, Berrien Springs, Michigan 49103, U.S.A.

I am told that data handling will be done at the Center for Statistical Services, School of Education, Andrews University. Each set of questionnaire is color coded to identify the five status groups by their job titles, not by their names or their institutions' names, for the purpose of the research. The subjects will not write their names or their institutions' names on the questionnaire or on the envelope. The procedures ensure the highest level of anonymity and confidentiality possible. 
Benefits/Results: I understand that I may not receive any direct benefits from participating in this study. The results may help the SDA college/university president in his/her professional role performance, strengthening Adventist education. The information collected during this study will be included in a doctoral dissertation, and may be presented or published in professional meetings or journals to further expand the understanding of the relationship between president's role performances and his/her role expectations.

Voluntary Participation: I have been advised that my participation in this study is completely voluntary and that I may refuse to participate in this research at any time without experiencing negative consequences, penalties or prejudice or denial of benefits to which I am entitled. I also understand that there is no compensation in return for my participation.

Risks and Discomforts: I understand that there are no risks, discomforts, or invasion of privacy during or as a result of my participation in the study. It does not cause damage to my financial standing, employability or reputation. The amount of stress is extremely minimal, if at all present.

Impartial Third Party: I have been told that if I wish to contact an impartial third party regarding any complaint that I may have about the study, I may contact Dr. Hinsdale Bernard, Program Coordinator, Educational Administration \& Leadership, School of Education, Andrews University, Berrien Springs, Michigan 49104, U.S.A. Phone: (616) 4716702. Fax (616) 471-6374. E-mail: <hbernard@andrews.edu>

Further Questions: I have been told that I can contact the researcher should I need further information, assistance or have any questions about the research.

I have read the contents of this consent form and have also read the approval as well as the instruction letters. My questions concerning this study have been answered to my satisfaction. I hereby give my voluntary consent to participate in this study. I know my anonymous return of the completed questionnaire to the researcher will serve as a form of implied consent to the statements in the above paragraphs and I will not be required to send this INFORMED CONSENT DOCUMENT back to the researcher.

$\overline{\text { Signature of Adult Research Participant }}$

Signature of Adult Witness
Date

Date

NOTE FROM RESEARCHER TO ADULT RESEARCH PARTICIPANT:

Please mail the original copy of the completed questionnaire today and keep this signed Informed Consent Document with you. Thank you. 


\section{Sunith K. Das}

September 20, 2004

Board Chairs, Presidents \& Supporting Administrators

Seventh-day Adventist Senior Colleges \& Universities

Subject: Informed Consent Letter

Dear Leaders:

Greetings in the name of our Lord!

A few days back I had mailed to you an envelope containing questionnaire, an endorsement letter from the General Conference Education Department, an informed consent document, and a cover letter from me, requesting for your kind participation in my doctoral research project. I'm sure you have received these documents by now.

Please DO NOT return the informed consent document back to me. It's for you to read, sign and keep. Just send me the completed questionnaire in the self-addressed postagestamped (applies to the U.S.A. and its territories only) envelope provided earlier. I apologize to you for any confusion that may have arisen from my previous communique.

If you have any further question, comment or observation relative to this approved research project, please let me know. I need your assistance to complete my dissertation.

Thank you once again for your support.

Sincerely,

Sunith K. Das

Ph.D. Candidate

Educational Administration \& Supervision, Andrews University

Copy to: $\quad$ Hinsdale Bernard, Ph.D., Research Advisor \& Dissertation Committee Chair, and Professor, Department of Leadership and Educational Administration, Andrews University. 
APPENDIX D

CHARACTERISTICS OF A SUCCESSFUL

SEVENTH-DAY ADVENTIST COLLEGE

OR UNIVERSITY 


\section{CHARACTERISTICS OF A SUCCESSFUL SEVENTH-DAY ADVENTIST COLLEGE OR UNIVERSITY}

The following characteristics distinguish a successful Seventh-day Adventist college or university:

1. Philosophy and Mission. It is a community of learners committed to the truth and guided by a statement of philosophy, mission, vision and objectives congruent with the Seventh-day Adventist message and mission, crafted jointly by administration, faculty, and board, and reflected in all the programs and activities of the institution.

2. Governance and Accountability. It is governed by a board that is representative of and accountable to the constituency. The board appoints administrators, faculty, and staff; develops institutional policies; approves the yearly budget; and advices the leadership as it administers the institution. Working through designated committees (for academics, finances, student development, etc.), the board interfaces with the administration and provides overall guidance. Once a year, the board receives from the administration a detailed evaluation of the extent to which the institution is achieving its academic and spiritual mission.

3. Administration. Its administrative team implements the philosophy and mission of the institution; fulfils the expectations of the board and the constituency; provides job descriptions that foster responsibility and accountability; carries out a non-discriminatory policy in the recruitment of staff and students within the framework of Adventist beliefs; promotes professional growth among faculty, staff, and students; and carefully plans for the future of the institution.

4. Faculty and Staff. It has a professionally qualified faculty and staff committed to the Seventh-day Adventist message, mission, and lifestyle, and to the education of young men and women for a useful life "in this world and in the world to come." The institution implements an initiation program for new faculty; promotion in academic rank follows a clear process.

5. Programs. Its educational offerings and co-curricular activities respond to the needs and expectations of the constituency, within the framework of the financial resources available. These programs aim at the harmonious development of young men and women in their mental, spiritual, physical, and social dimensions in order that they may achieve academic and professional excellence.

6. Students. Young men and women studying at the institution are encouraged to accept Christ as their Savior, to develop Christian characters, to engage in a life-long search for a 
deeper understanding of and commitment to God's truth, to support the mission of the church, to exhibit lifestyle consistent with biblical values, and to become contributors to the well being of society.

7. Spiritual Life. It has implemented a campus-wide spiritual master plan to promote and assess the transmission of Biblical-Christian beliefs, principles, and values to the students and, in cooperation with faculty and staff, provides opportunities for the institutional family to become involved in devotional life, religious nurture, evangelistic outreach, and service for the community.

8. Financial Resources. It pursues careful stewardship and management of resources entrusted by the constituency, and has an adequate financial administration covering faculty and staff remuneration, tuition, scholarships, insurance, industries, development, and reserves. It strengthens its financial base by involving benefactors in the raising of resources for plant development, scholarships, research, and service. Accounts are up-todate and verified through regular audit reports.

9. Infrastructure, Equipment, and Services. Its human resources and physical infrastructure provide services supportive of the institutional objectives, programs, and activities. The infrastructure includes adequate facilities and services such as cafeteria, classrooms, counseling, cultural programs, health care, laboratories and computing facilities, library, residences, security, sports, store, and student labor. The campus atmosphere projects a wholesome view of Seventh-day Adventist aesthetics and principles.

10. Communication. It utilizes print, audio-visual, and electronic media to describe its programs, activities, and plans; to foster internal information flow; to make available to the wider community the results of faculty and students' research; and to present a positive image of the institution.

11. Recruitment and Follow-Up. It attracts qualified students - especially from within the constituency - through a dynamic recruitment program, assists them in obtaining suitable employment after graduation, seeks their input for future planning, and cultivates their support through regular alumni activities.

12. Recognition and Accreditation. Its academic/professional programs are recognized and approved by denominational bodies (International Board of Education and Adventist Accrediting Association) and by governmental/regional accrediting entities. The institution holds current denominational accreditation.

Adopted by the Education Department directors attending the World Education Advisory at the General Conference Headquarters - April 1996 - Revised March 1997. 
APPENDIX E

PRELIMINARY RESEARCH INSTRUMENT STUDY 


\section{PARTICIPANTS IN THE RESEARCH INSTRUMENT VALIDATION STUDY}

\begin{tabular}{|c|c|c|c|c|c|c|}
\hline $\begin{array}{l}\text { Name of Institution } \\
\text { Represented }\end{array}$ & $\begin{array}{l}\text { Chairman of } \\
\text { Board of } \\
\text { Trustees }\end{array}$ & $\begin{array}{l}\text { College / } \\
\text { University } \\
\text { President }\end{array}$ & $\begin{array}{c}\text { VP for } \\
\text { Academic } \\
\text { Administration }\end{array}$ & $\begin{array}{l}\text { VP for } \\
\text { Student } \\
\text { Life }\end{array}$ & $\begin{array}{l}\text { VP for } \\
\text { Financial } \\
\text { Service }\end{array}$ & Total \\
\hline $\begin{array}{l}\text { Andrews University } \\
\text { Michigan, U.S.A. }\end{array}$ & 1 & 1 & 1 & 2 & 2 & 7 \\
\hline $\begin{array}{l}\text { Bangladesh Adventist College } \\
\text { Goalbathan, Bangladesh }\end{array}$ & & 1 & & 1 & & 2 \\
\hline $\begin{array}{l}\text { Columbia Union College } \\
\text { Maryland, U.S.A. }\end{array}$ & & & 1 & & & 1 \\
\hline $\begin{array}{l}\text { Helderberg College } \\
\text { Cape, South Africa }\end{array}$ & & $1^{*}$ & & & & 1 \\
\hline $\begin{array}{l}\text { Adventist International Institute } \\
\text { of Advanced Studies } \\
\text { Manila, Philippines }\end{array}$ & & 1 & & & & 1 \\
\hline $\begin{array}{l}\text { Montemorelos University } \\
\text { Montemorelos, N.L., Mexico }\end{array}$ & & & 1 & & & 1 \\
\hline $\begin{array}{l}\text { Solusi University } \\
\text { Zimbabwe, Africa }\end{array}$ & & & & & 1 & 1 \\
\hline $\begin{array}{l}\text { Southeast Asia Union College } \\
\text { Singapore }\end{array}$ & & & & 1 & & \\
\hline Total & 1 & 4 & 3 & 4 & 3 & $N=15$ \\
\hline
\end{tabular}

*No data 


\section{Sunith K. Das}

\section{Kimber Lane, Berrien Springs, Michigan 49103, U.S.A.}

Dear Fellow Educator(s):

I am a Ph.D. candidate in Educational Administration and Supervision in the School of Education at Andrews University. I am preparing a dissertation on the role expectation of Seventh-day Adventist senior college and university presidents worldwide as perceived by the chairpersons of boards of trustees, presidents, vice presidents for academic administration, vice presidents for student service, and vice presidents for finance. The General Conference Education Director has endorsed this study.

I need to pilot test my research instrument before sending the questionnaire to the subjects selected from SDA senior colleges and universities around the world. Therefore, I am sending this questionnaire to the individuals who are experienced in higher education administration and in a position to critique it as an instrument. I am requesting you to kindly fill out the questionnaire and then answer the following questions:

1. How long did it take you to complete the questionnaire?

2. Are the questions clear?

3. Are the questions relevant to the study?

4. What portion(s) of the questionnaire should be deleted?

5. What new question(s) should be added?

6. What are your overall opinions, views, and suggestions?

You can provide your responses on the questionnaire sheets. I know you are an extremely busy person, but please share a few moments to take part in this pilot test procedure. I can collect your responses personally from you or you can send them to the Graduate Dean's Office through campus inter-mail addressed to me. Thank you for your support of this important study.

Sincerely,

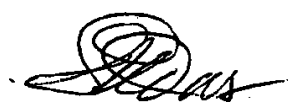

Sunith K. Das

Enclosures 


\section{Perceptions and Expectations of Seventh-day Adventist College and University Presidents' Roles}

From the Views of

Board Chairs, Presidents, Academic Vice Presidents, Student Service Vice Presidents, and Finance Vice Presidents of Seventh-day Adventist College and University Presidents,

Purpose: The purpose of this questionnaire is to obtain data which will reveal your expectations and perceptions regarding your role and function as president of Seventh-day Adventist (SDA) university or senior college. From this information, concurring and/or conflicting views may appear and an awareness of these views may assist in ameliorating some turmoil in college and university administration. The data will be used for a purpose beyond dissertation problem resolution.

Significance: Your response will contribute significantly to the study of SDA college and university administration by clarifying the role and functions of the president and his/her relationship with the board of trustees and supporting administrators.

Procedure: This is a modified instrument developed from the Oliver (1972) and Silas (1974) instruments which were earlier tested by several college and university presidents, academic deans, governing board members; a "pre-test" among faculty and advanced doctoral students who were experienced in university administration; and a "pilot study" among college and university presidents, academic deans, and board members.

Direction: The questionnaire has two parts which should take a total of twenty minutes to complete. Your participation in this study is greatly appreciated. Confidentiality will be maintained. Neither you nor your institution will be identified with any individual versus group data determined from this set of questionnaire.

After you have completed the questionnaire, please place it in the enclosed, selfaddressed, postage stamped envelope and return it to the researcher named below. Each set of questionnaire is color coded for mailing, follow-up and data processing.

Thank you for your cooperation and support.

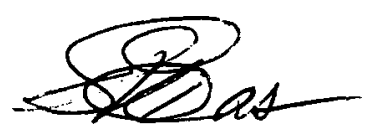

Sunith K. Das

Researcher

c/o Dr. Lyndon G. Furst, Chair, Dissertation Committee, Educational Administration, School of Education, Andrews University, Berrien Springs, Michigan 49104, U.S.A. 


\section{PART I. DEMOGRAPHIC DATA}

1. Male

Female

2. Age

3. Check below the item which describes the last institutional position you held prior to the current presidency:
a. Academic Vice President/Academic Dean
b. Vice President for Student Services/Dean of Students
c. Vice President for Finance/Business Manager
d. Teacher
e. Other

4. Name of institution represented

5. Check the slot which indicates if you have held an administrative position any time prior to acceptance of the presidency.

$$
\text { Yes__ No }
$$

6. Your highest degree earned

7. Field in which your degree was awarded

8. Year of degree award

9. Name of institution degree was earned

10. Years in present position

11. Name of institution prior to presidency

12. Identify the range of your faculty size (FTE equivalent):

less than 50

$51-100$ 
$100-200$

201-300

$300-400$

Over 400

\section{PART II. ROLE PERFORMANCE AND ROLE EXPECTATION DATA}

The purpose of this section is to elicit your disagreement or agreement with various possible aspects of a president's role and functions. The range is from 1 (Strongly Disagree) to 5 (Strongly Agree). Please circle the numeral that best reflects your view. In the grid marked Expected Role Responsibility please indicate the extent to which you expect responsibility in these areas. In other words, if you feel that the item should belong to a president's area of responsibility, or if you expect to perform it in your capacity as president, please indicate the strength of that expectation. Rank each of these activities on the scale by assigning it a rating of 1 if you do not expect to perform this function and $\underline{5}$ if you definitely expect to perform this responsibility. Assign values of $\underline{2}$, $\underline{3}$, or $\underline{4}$ for intermediate degrees of expectation. Circle the numeral which you feel applies.

In the grid marked Perceived Role Performance please indicate whether or not you actually do perform these functions. Rank each of these perceptions on the scale following the activity by assigning it a rating of 1 if you do not perform this function and $\underline{5}$ if you have significant responsibility for its performance. Assign values of $\underline{2}, \underline{3}$, or $\underline{4}$ for intermediate responsibility for performance. Circle the numeral which indicates your perception for actual role performance. 


\section{STUDENT-RELATED FUNCTIONS}

Expected Role

Responsibility

\begin{tabular}{|c|c|c|c|c|}
\hline 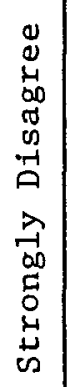 & 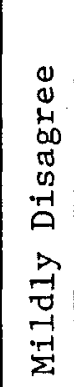 & $\begin{array}{l}5 \\
. \\
.7 \\
.7 \\
0 \\
0 \\
0 \\
2\end{array}$ & 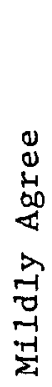 & 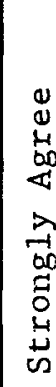 \\
\hline 1 & 2 & 3 & 4 & 5 \\
\hline 1 & 2 & 3 & 4 & 5 \\
\hline 1 & 2 & 3 & 4 & 5 \\
\hline 1 & 2 & 3 & 4 & 5 \\
\hline 1 & 2 & 3 & 4 & 5 \\
\hline 1 & 2 & 3 & 4 & 5 \\
\hline 1 & 2 & 3 & 4 & 5 \\
\hline 1 & 2 & 3 & 4 & 5 \\
\hline
\end{tabular}

Perceived Role

Performance

DIRECTION: Circle one numeral on each grid indicating your perceptions and expectations of president's role.

The university or college president should:

1. participate in student recruitment

2. work closely with student government leaders

3. be frequently available to students for dialogue

4. visit students in the dorms

5. be responsible for student discipline

6. teach classes to have some contact with the students

7. know every student personally

8.

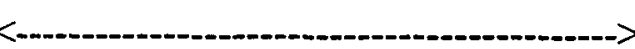

\begin{tabular}{|c|c|c|c|c|}
\hline 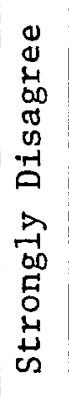 & 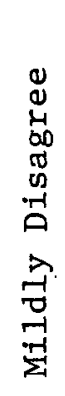 & 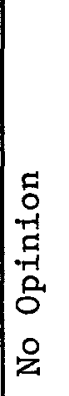 & 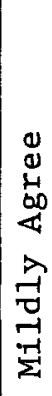 & 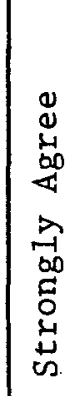 \\
\hline 1 & 2 & 3 & 4 & 5 \\
\hline 1 & 2 & 3 & 4 & 5 \\
\hline 1 & 2 & 3 & 4 & 5 \\
\hline 1 & 2 & 3 & 4 & 5 \\
\hline 1 & 2 & 3 & 4 & 5 \\
\hline 1 & 2 & 3 & 4 & 5 \\
\hline 1 & 2 & 3 & 4 & 5 \\
\hline 1 & 2 & 3 & 4 & 5 \\
\hline
\end{tabular}




\section{FACULTY-RELATED FUNCTIONS}

- Expected Role

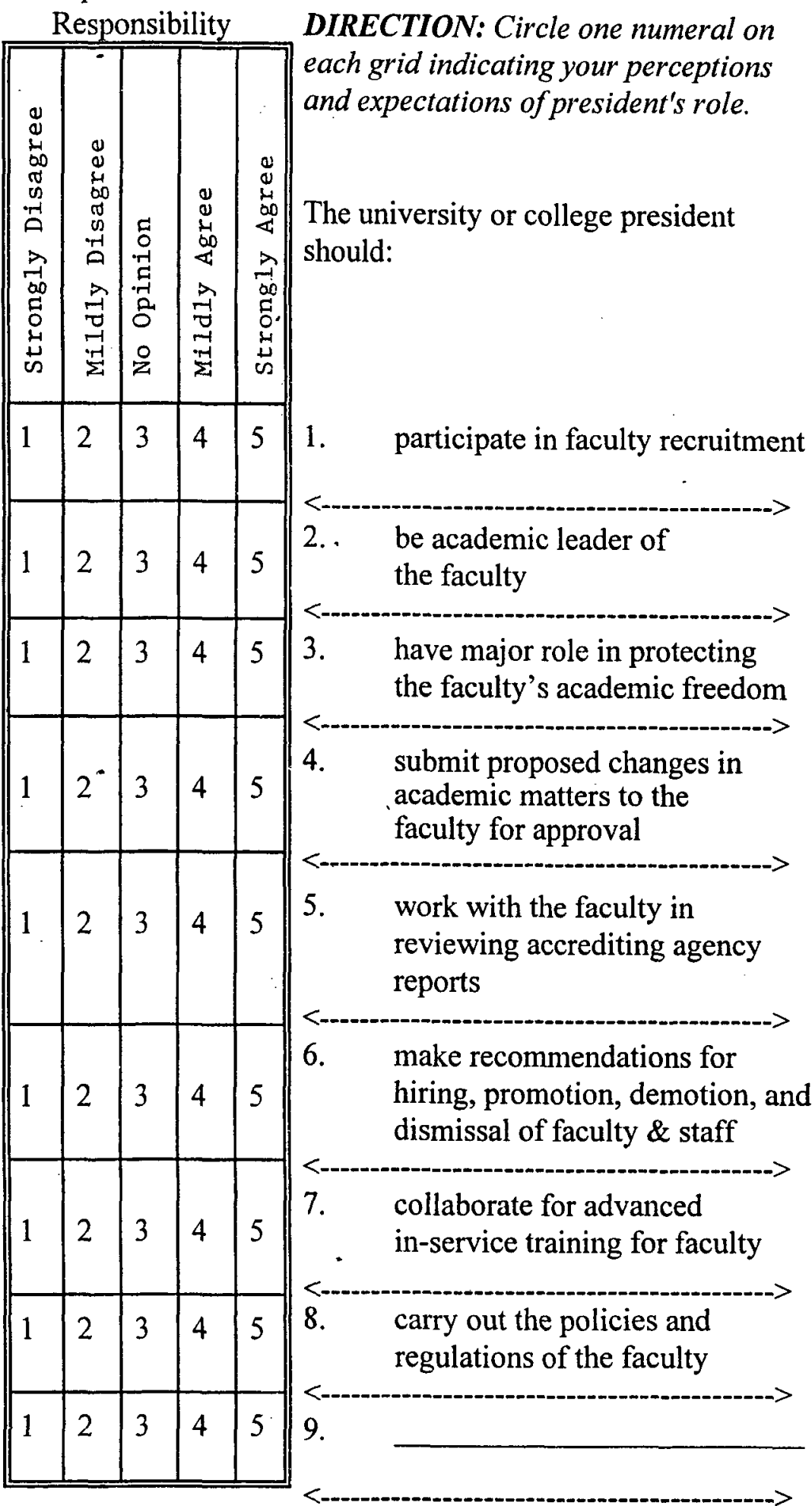

Perceived Role

Performance

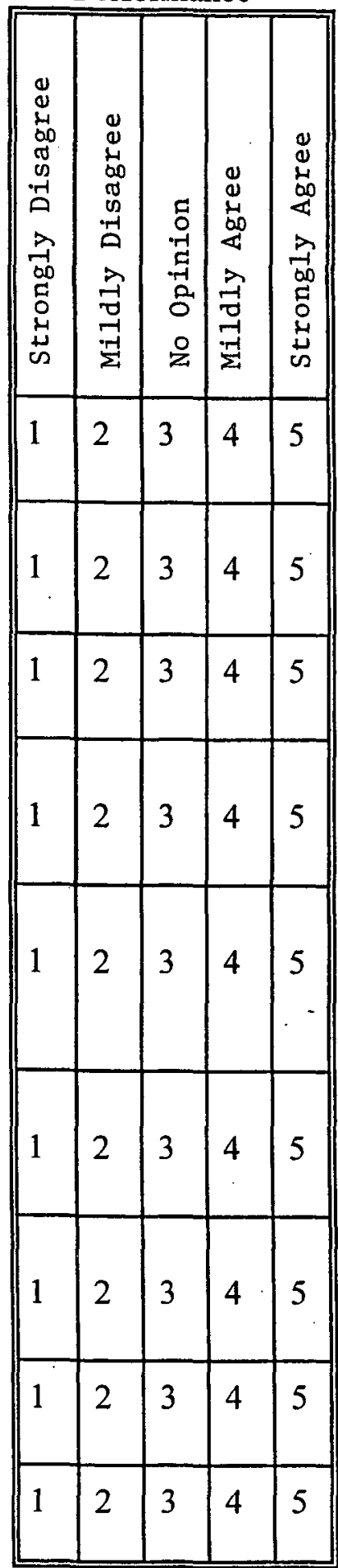


Expected Role

Responsibility

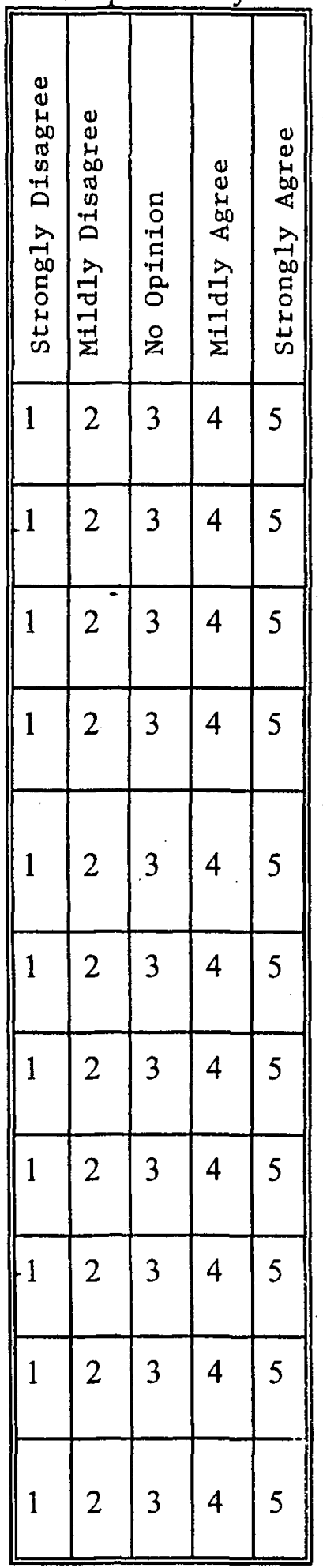

GENERAL ADMINISTRATION

DIRECTION: Circle one numeral on each grid indicating your perceptions and expectations of president's role.

The university or college president should:

1. prepare agenda for board meetings

2. preside at general faculty meetings <--.-.-.-.-.- > 3. be responsible for structuring admission standards

4. bear responsibility for satisfactory governance of the institution

5. serve as an ex-officio member of all faculty committees

6. prepare and revise the chart of administrative organization

7. assign officers their roles <.-.--

8. be in charge of planning for the future of the institution

9. formulate institution's educational philosophy

10. serve as chief disciplinary officer 11.
Perceived Role

Performance

\begin{tabular}{|c|c|c|c|c|}
\hline 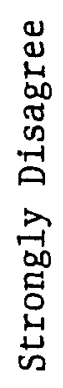 & 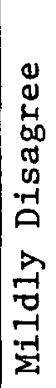 & 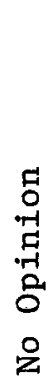 & 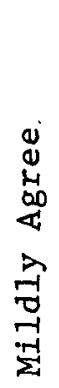 & 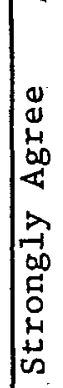 \\
\hline 1 & 2 & 3 & 4 & 5 \\
\hline 1 & 2 & 3 & 4 & 5 \\
\hline 1 & 2 & 3 & 4 & 5 \\
\hline 1 & 2 & 3 & 4 & 5 \\
\hline 1 & 2 & 3 & 4 & 6 \\
\hline 1 & 2 & 3 & 4 & 5 \\
\hline 1 & 2 & 3 & 4 & 5 \\
\hline 1 & 2 & 3 & 4 & 5 \\
\hline 1 & 2 & 3 & 4 & 5 \\
\hline 1 & 2 & 3 & 4 & 5 \\
\hline 1 & 2 & 3 & 4 & 5 \\
\hline
\end{tabular}


FINANCE-RELATED FUNCTIONS

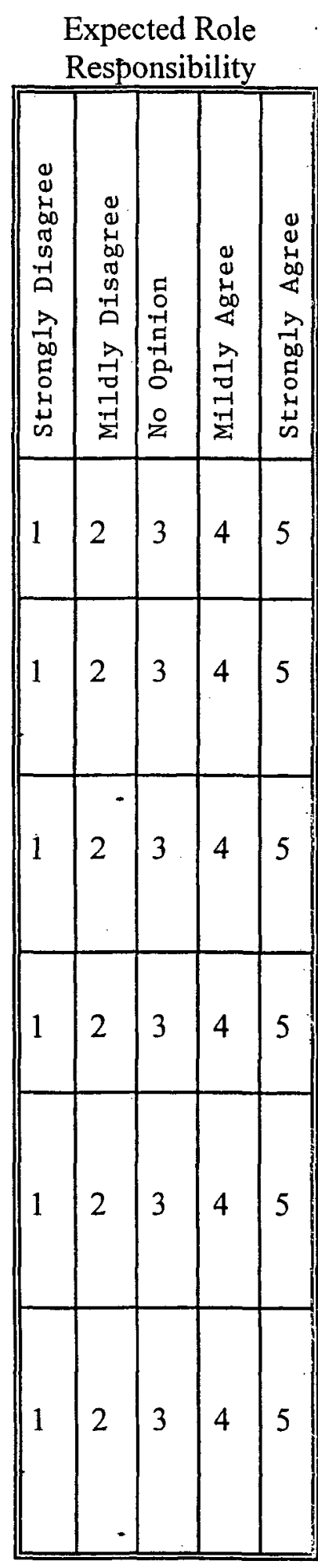

DIRECTION: Circle one numeral on each grid indicating your perceptions and expectations of president's role.

The university or college president should:

1. assist in the preparation of annual budget for the institution and present it to the board

2. see that the college budget, after approval by the board, is adhered to

3. present the annual audited financial statement to the board of trustees

4. recommend salary changes to the board

5. be held accountable to the board for financial operation of the institution

6.
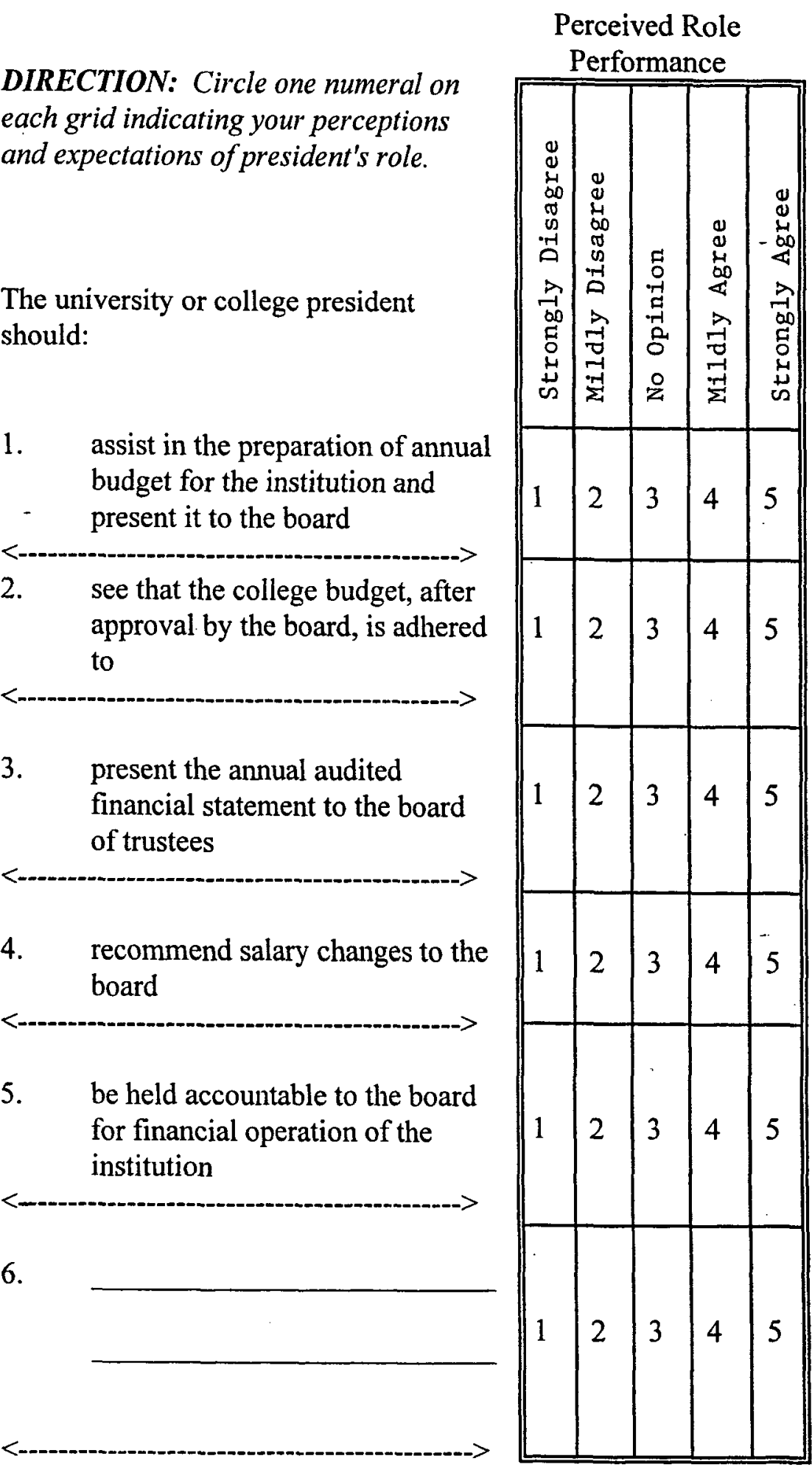
Expected Role

Responsibility

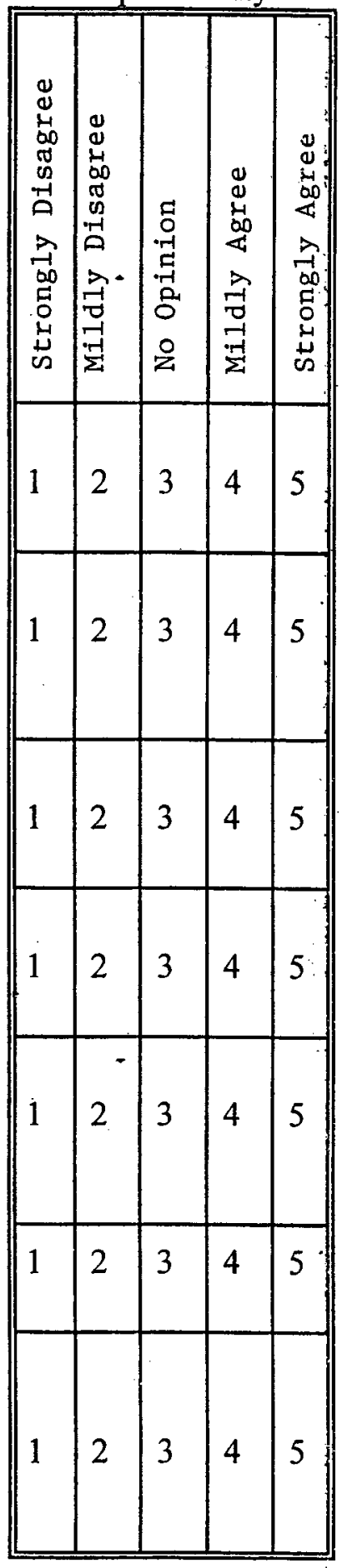

\section{PHYSICAL FACILITIES-RELATED FUNCTIONS}

DIRECTION: Circle one numeral on each grid indicating your perceptions and expectations of president's role.

The university or college president should:

1. present to the board the physical needs of the institution

2. direct the preparation of plans for new buildings and installations as needed

3. plan for major repairs or

- alterations of plant, buildings, and equipment

4. recommend to the board for equipment

5. ensure that physical plant master plans are kept up to date and followed as closely as possible

6. make sure that campus grounds are well-kept

7.

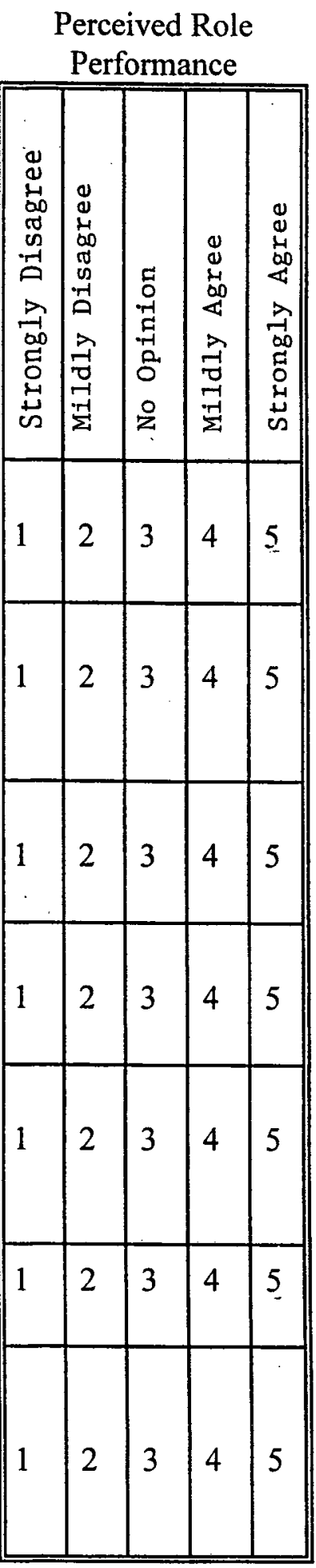


Expected Role

Responsibility

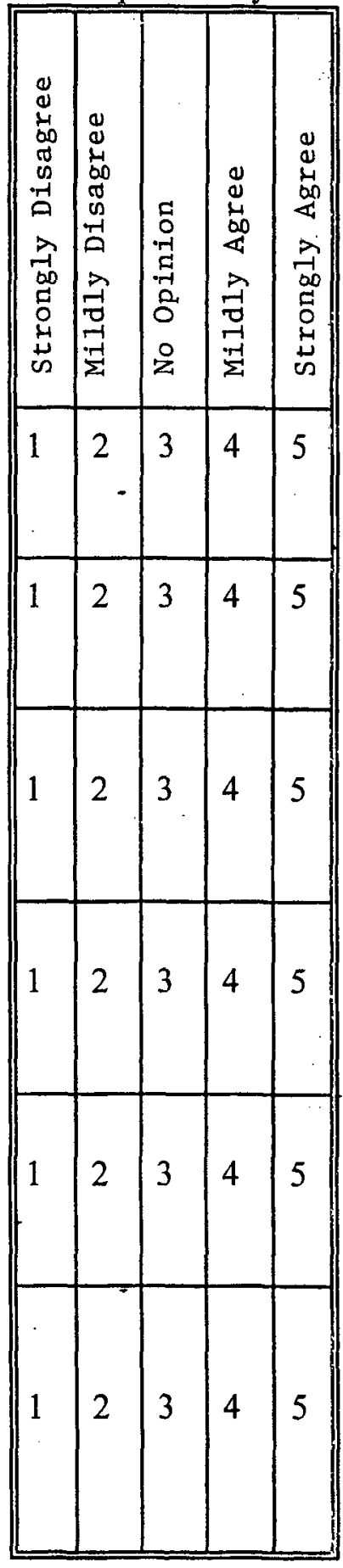

COMMUNITY RELATIONS AND ALUMNI RELATED FUNCTIONS

DIRECTION: Circle one numeral on each grid indicating your perceptions and expectations of president's role.

The university or college president should:

1. assist in raising funds for the support of the college

2. represent the college in the churches of the constituency

3. prepare reports concerning the college and present these reports to the community

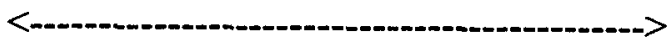

4. represent the college as its official spokesperson to the public

$<>$

5. be closely associated with alumni groups

6. 
SPIRITUAL LIFE-RELATED

FUNCTIONS

Expected Role

Responsibility

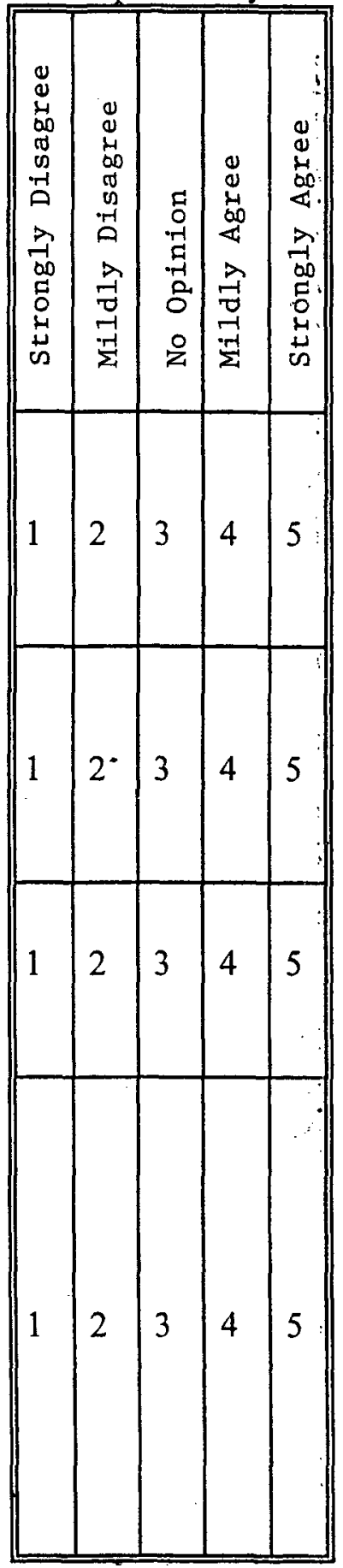

DIRECTION: Circle one numeral on each grid indicating your perceptions and expectations of president's role.

The university or college president should:

1. assume primary responsibility for the promotion of the religious life of the students and faculty

Perceived Role

Performance

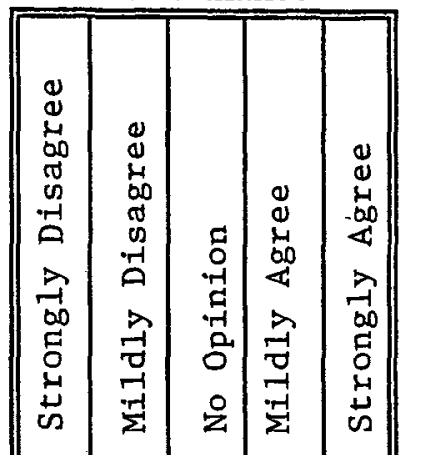

2. be responsible for chapels, vespers, weeks of spiritual emphasis, and other religious programs

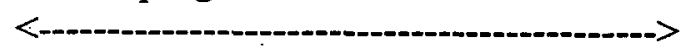

3. manifest Christ-like character in spiritual leadership roles

4.
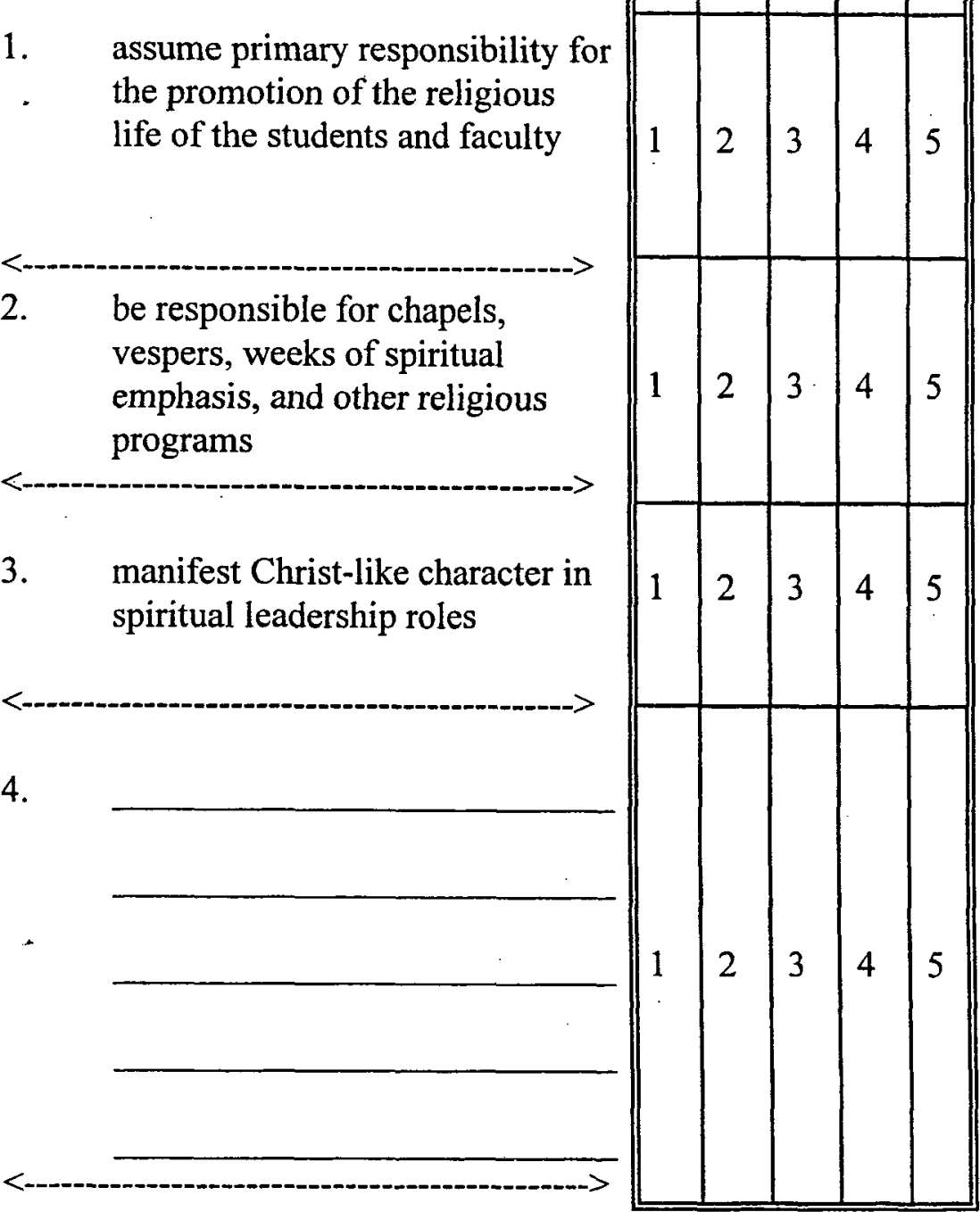


\section{SAMPLE SUGGESTIONS, COMMENTS, AND VIEWS RECEIVED FROM PRELIMINARY RESEARCH INSTRUMENT VALIDATION STUDY PARTICIPANTS}

1. "May need an item on collaborative function."

2. "Perhaps include HR functions."

3. "In a decentralized institution president is responsible but rely on V.P. officer to execute."

4. "Other important functions:

Strategic planning

Legal transactions

Grievances and resolutions

Compliance with legal mandates \& laws

Foster research \& intellectual climate

Listen to constituents"

5. "Delegate authority \& needed resources to appropriate administrators."

6. "Support administrators in their roles."

7. "You may want to consider a separate category saying "Not enough knowledge to give an opinion" or "Not applicable" instead of "no opinion."

8. "This depends much on the size of the institution."

9. "I have suggested some changes but find the questionnaire quite reasonable and comprehensive."

10. "Recruiting of students in small college is different from recruiting students in big institutions like A.U. In big institutions the presidents cannot participate in the recruiting process."

11. Serve as an ex-officio member of all faculty committees. "Varies from small to big institutions."

12. "There are institutions where the Business Manager is sole authority of financial matters and the presidents have no function in it." 
13. "This is an excellent instrument."

14. "The functions seem appropriate but are too much for one individual. Perhaps the questions should refer to the president or his/her designee.

15. In a school such as A.U. it is difficult to answer the questions the way they are posed. So much is delegated to various VPs. The president must be aware and ensure that many aspects of institution are followed through with or be involved but must delegate and only give direction.

16. "The questions are adequate, relevant and easily understood.

17. Recommend to the board for equipment. "When it is a major purchase, yes." 


\section{Sample Responses from the Participants to Questions Asked by the Researcher of this Study Regarding the Preliminary Research Instrument}

1. How long did it take you to complete the questionnaire? 30 minutes

2. Are the questions clear? One problem I faced in responding to the instrument was the inclusion of too many items in one question. Under Faculty Related Functions, item \#6, hiring, promotion, demotion, and dismissal of faculty and staff, which really makes 8 points, is much too broad for me to answer as it relates to Andrews University. Or, under Finance Related Functions, item \#1 is really 2 questions. The first one is easy because I assisted in budget preparation, but I did not present it to the board because there was a vice president for finance, and although I introduced him, he presented the budget, but only by introducing the controller who made the actual presentation. On the same page item \#4 was not clear because I did not know what salary changes you had in mind. For individuals these were by policy, but if you are speaking of salary scale then of course it was a Board change. Also, I was puzzled by some questions that did not seem to clarify whether the responsibility in question was an ultimate responsibility that was actually carried out by others or if it was a direct responsibility that was carried out by the president himself. Another problem I faced was this: I can understand how one can have no opinion about an expected role, but I don't see how it is possible to have no opinion about one's own performance. Perhaps there ought to be a forced choice with only four columns, at least on the performance side. It also needs to be kept in mind that the responsibilities that are to be directly fulfilled by the president varies according to the number of vice presidents the institution has.

I suggest you include a question on the preparation of the institutional report to the accrediting agency, and on the institutional working policy.

3. Are the questions relevant to the study? Yes, except as I noted above regarding No Opinion on perceived role performance.

4. What portion of the questionnaire should be deleted? Only as may be required by above item.

5. What new questions should be added? Sorry, I moved into this question under \#2.

6. What are your overall opinion, views, and suggestions regarding the instrument? In my opinion the titles on the two grids give a wrong impression. It reads as though you are comparing an expectation and a perception, but it seems to me you are intending to compare an expectation and a performance. To convey that accurately I think you should be comparing Expected Role Responsibility and Performance Role Perception. 
APPENDIX F

MODIFIED RESEARCH INSTRUMENT

AND INSTRUCTIONS 
Questionnaire and Instructions

for Presidents 


\section{Role Performance and Role Expectation \\ Of Seventh-day Adventist Senior College and University Presidents As Perceived by Seventh-day Adventist Senior College/University's \\ Presidents Themselves}

Purpose of Questionnaire: The purpose of this questionnaire is to obtain data which will reveal the expectations and perceptions regarding your role and function at the Seventhday Adventist (SDA) university or senior college where you are serving as president. This study will investigate concerning levels of commons agreement and disagreement between respondent groups on actual and expected roles of SDA senior college and university presidents. The data will be used for a purpose beyond dissertation problem resolution. Your response will contribute significantly to the study of SDA college and university administration by clarifying your role and function as president and your relationship with the supporting administrators.

Direction for Questionnaire: The questionnaire has 47 statements listed under 7 broad categories of functions with one open slot in each for you to add any activity you feel should be included. Rank each activity on a continuum scale ranging from 1 (Strongly Disagree) to 5 (Strongly Agree). Circle the numeral that best reflects your view.

In the first grid, titled, Actual Role Performance, indicate the extent to which you feel you as president are presently performing your role. Rank each of these perceptions on the scale following the activity by assigning it a rating of 1 , if you do not perform this function, and 5, if you demonstrate significant responsibility for its performance. Assign values of 2,3 , or 4 for intermediate responsibility for performance. Circle only one numeral for each activity which indicates your perception for your present actual role performance.

In the second grid, titled, Expected Role Responsibility, indicate the extent to which you expect that the item of function should belong to your area of responsibility, or you expect to perform it in the capacity of president. Rank each of these activities on the scale following the activity by assigning it a rating of 1 , if you do not expect to perform these functions, and 5 , if you strongly agree that you should perform these responsibilities. Assign values of 2,3 , or 4 for intermediate degrees of expectation. Circle only one numeral for each activity which you feel applies.

Please respond to all items of the seven sections in the two grids. Anonymity and confidentiality will be maintained. Do not write your or your institution's name anywhere. After you have completed the questionnaire, please place it in the enclosed self-addressed postage-stamped envelope and mail it today. If you have any question, please contact the researcher or the research advisor. Thank you for participating in this research project.

Researcher: Sunith K. Das, 4775 Kimber Lane, Berrien Springs, MI 49103. Phone: (269) 473-1612. E-mail: $\leq$ dass@andrews.edu $>$

Research Advisor \& Dissertation Committee Chair: Hinsdale Bernard, Ph.D., Professor of Education, Andrews University, Berrien Springs, MI 49104. Phone (269) 471-6702. E-mail: <hbernard@andrews.edu $>$ 


\section{STUDENT-RELATED FUNCTIONS}

In this section you are asked about your perception of your present actual role performance and expected role responsibility in your student-related functions as Seventh-day Adventist senior college/university president. Indicate your degree of agreement or disagreement with each statement by circling one number for each in both the grids. The scale: 1=you strongly disagree (SD), 2=you disagree (D), 3=you neither agree nor disagree (N), 4=you agree (A), and 5=you strongly agree (SA).

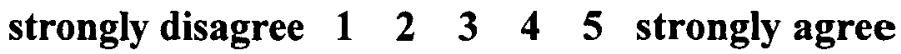

As Seventh-day Adventist senior college/ university president you do and should:

1. Participate in student recruitment

2. Work closely with student government leaders

3. Dialogue frequently with students

4. Visit students periodically in dorms during dorm worships

5. Take ultimate responsibility for student discipline

6. Teach some classes in order to maintain contact with students

7. Know every student personally

8. Serve as mentor of students

Actual Role

Performance

(You Do)

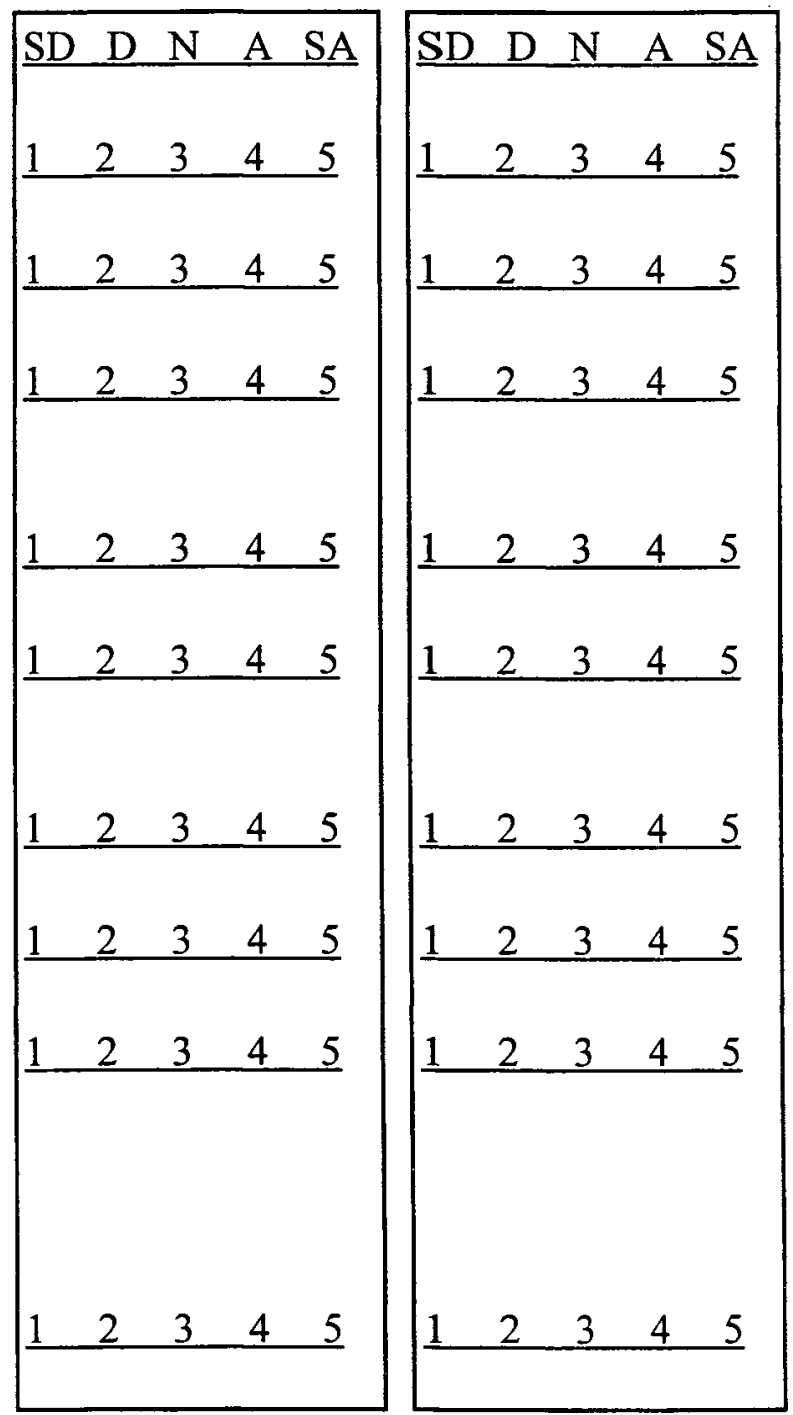

(Optional: One open-ended question here) 


\section{EMPLOYEE-RELATED FUNCTIONS}

In this section you are asked about your perception of your present actual role performance and expected role responsibility in your employee-related function as Seventh-day Adventist senior college/university president. Indicate your degree of agreement or disagreement with each statement by circling one number for each in both the grids. The scale: 1=you strongly disagree (SD), 2=you disagree (D), 3=you neither agree nor disagree $(\mathrm{N}), 4=$ you agree (A), and 5=you strongly agree (SA).

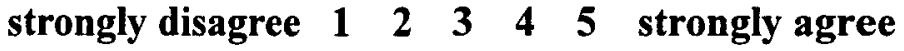

Actual Role

Performance

(You Do)

As Seventh-day Adventist senior college/ university president you do and should:

9. Participate in faculty recruitment

10. Serve as academic leader of faculty

11. Guard faculty's academic freedom

12. Have responsibility in submitting proposed changes in academic matters to faculty for approval

13. Work with faculty in reviewing accrediting agency's reports

14. Recommend to board for hiring, promotion, demotion, and dismissal of faculty and staff

15. Collaborate for advanced inservice training for faculty \& staff

16. Carry out policies and regulations of faculty and staff

(Optional: One open-ended question here)

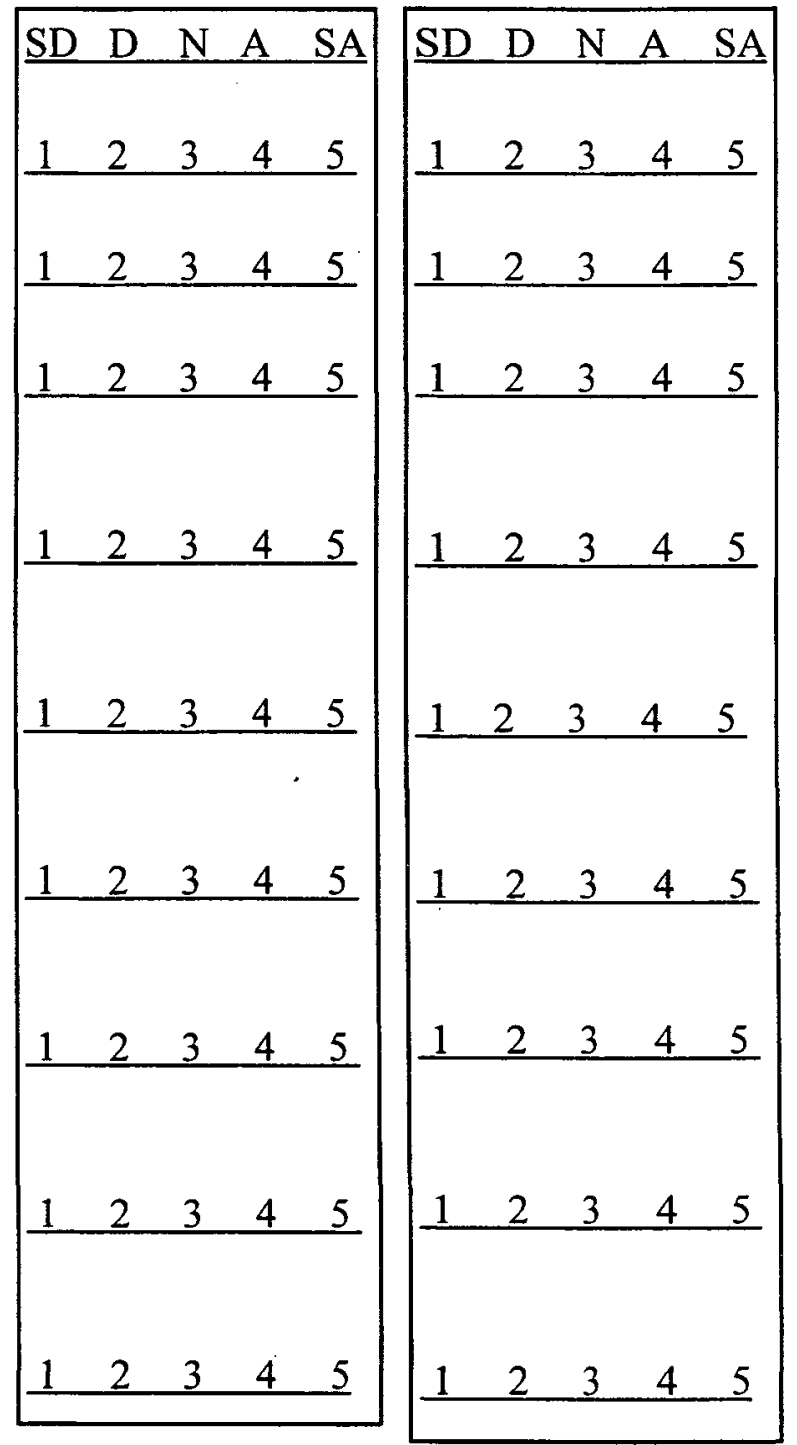




\section{GENERAL ADMINISTRATIVE FUNCTIONS}

In this section you are asked about your perception of your present actual role performance and expected role responsibility in your general administrative functions as Seventh-day Adventist senior college/university president. Indicate your degree of agreement or disagreement with each statement by circling one number for each in both the grids. The scale: 1=you strongly disagree (SD), 2=you disagree (D), 3=you neither agree nor disagree $(\mathrm{N}), 4=$ you agree (A), and 5=you strongly agree (SA).

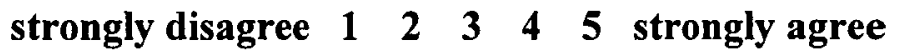

\begin{tabular}{|c|c|c|c|}
\hline \multicolumn{2}{|c|}{$\begin{array}{l}\text { As Seventh-day Adventist senior college/ } \\
\text { university president you do and should: }\end{array}$} & $\begin{array}{l}\text { Actual Role } \\
\text { Performance } \\
\text { (You Do) }\end{array}$ & $\begin{array}{l}\text { Expected Role } \\
\text { Responsibility } \\
\text { (You Should) }\end{array}$ \\
\hline 17. & $\begin{array}{l}\text { Prepare agenda for board of trustees' } \\
\text { meetings }\end{array}$ & \begin{tabular}{|lllll} 
SD & D & N & A & SA \\
1 & 2 & 3 & 4 & 5 \\
\end{tabular} & \begin{tabular}{|lllll}
$\mathrm{SD}$ & $\mathrm{D}$ & $\mathrm{N}$ & $\mathrm{A}$ & $\mathrm{SA}$ \\
1 & 2 & 3 & 4 & 5 \\
\end{tabular} \\
\hline 18. & Preside over general faculty meetings & $1 \quad 2 \quad 3 \quad 4 \quad 5$ & $\begin{array}{lllll}1 & 2 & 3 & 4 & 5 \\
\end{array}$ \\
\hline 19. & $\begin{array}{l}\text { Take responsibility } \\
\text { student admission }\end{array}$ & $\begin{array}{lllll}1 & 2 & 3 & 4 & 5 \\
\end{array}$ & $\begin{array}{lllll}1 & 2 & 3 & 4 & 5 \\
\end{array}$ \\
\hline 20. & $\begin{array}{l}\text { Take responsibility in strategic } \\
\text { planning for institution }\end{array}$ & $\begin{array}{lllll}1 & 2 & 3 & 4 & 5 \\
\end{array}$ & $\begin{array}{lllll}1 & 2 & 3 & 4 & 5 \\
\end{array}$ \\
\hline 21. & $\begin{array}{l}\text { re, revise } \\
\text { hart }\end{array}$ & $\begin{array}{lllll}1 & 2 & 3 & 4 & 5 \\
\end{array}$ & $\begin{array}{lllll}1 & 2 & 3 & 4 & 5 \\
\end{array}$ \\
\hline 22. & atellectual & $\begin{array}{lllll}1 & 2 & 3 & 4 & 5 \\
\end{array}$ & $\begin{array}{lllll}1 & 2 & 3 & 4 & 5 \\
\end{array}$ \\
\hline 23. & $\begin{array}{l}\text { Com } \\
\text { laws }\end{array}$ & $\begin{array}{lllll}1 & 2 & 3 & 4 & 5 \\
\end{array}$ & $\begin{array}{lllll}1 & 2 & 3 & 4 & 5 \\
\end{array}$ \\
\hline 24. & $\begin{array}{l}\text { ere to } \\
\text { I philosophy }\end{array}$ & $\begin{array}{lllll}1 & 2 & 3 & 4 & 5 \\
\end{array}$ & $\begin{array}{lllll}1 & 2 & 3 & 4 & 5 \\
\end{array}$ \\
\hline 25. & $\begin{array}{l}\text { Delegate responsibility, authority, } \\
\text { and needed resources to appropriate } \\
\text { administrators and support their roles }\end{array}$ & $\begin{array}{lllll}1 & 2 & 3 & 4 & 5 \\
\end{array}$ & $\begin{array}{lllll}1 & 2 & 3 & 4 & 5 \\
\end{array}$ \\
\hline \multicolumn{2}{|c|}{ (Optional: One open-ended question here) } & & \\
\hline & & $\begin{array}{lllll}1 & 2 & 3 & 4 & 5 \\
\end{array}$ & $\begin{array}{lllll}1 & 2 & 3 & 4 & 5 \\
\end{array}$ \\
\hline
\end{tabular}




\section{FINANCE-RELATED FUNCTIONS}

In this section you are asked about your perception of your present actual role performance and expected role responsibility in your finance-related functions as Seventh-day Adventist senior college/university president. Indicate your degree of agreement or disagreement with each statement by circling one number for each in both the grids. The scale: 1=you strongly disagree (SD), 2=you disagree (D), 3=you neither agree nor disagree $(\mathrm{N}), 4=$ you agree $(\mathrm{A})$, and $5=$ you strongly agree (SA).

\section{$\begin{array}{llllllll}\text { strongly disagree } & 1 & 2 & 3 & 4 & 5 & \text { strongly agree }\end{array}$}

As Seventh-day Adventist senior college/ university president you do and should

26. Participate in the preparation of annual budget of institution

27. Ensure that institution's budget, after approval by board, is adhered to

28. Present institution's annual audited financial statements to board

29. Recommend to board the salary changes for positions

30. Accept accountability to board for financial operation of institution

(Optional: One open-ended question here)
Actual Role Performance

(You Do)

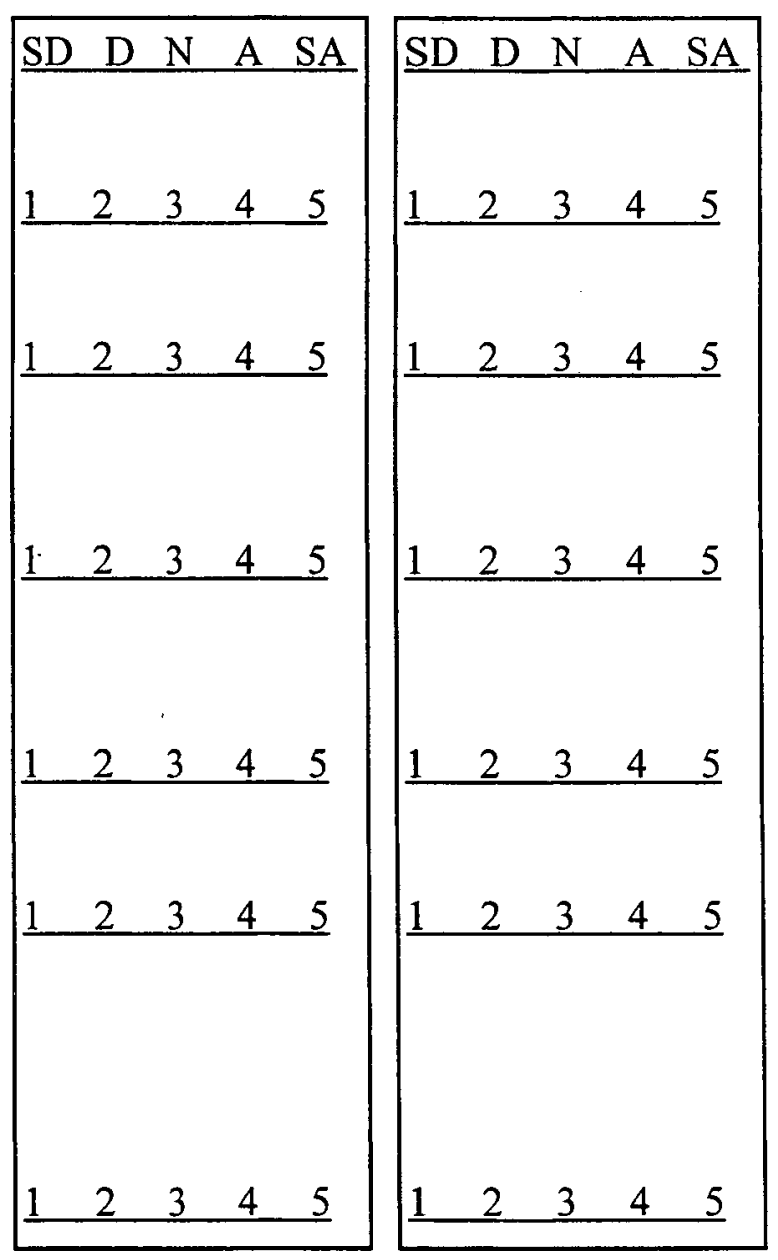




\section{PHYSICAL FACILITIES-RELATED FUNCTIONS}

In this section you are asked about your perception of your present actual role performance and expected role responsibility in your physical facilities-related functions as Seventhday Adventist senior college/university president. Indicate your degree of agreement or disagreement with each statement by circling one number for each in both the grids. The scale: 1=you strongly disagree (SD), 2=you disagree (D), 3=you neither agree nor disagree $(\mathrm{N}), 4=$ you agree $(\mathrm{A})$, and $5=$ you strongly agree (SA).

\section{strongly disagree $\quad 1 \quad 2 \quad \begin{array}{lllll}1 & 2 & 4 & 5 & \text { strongly agree }\end{array}$}

As Seventh-day Adventist senior college/ university president you do and should

31. Present to board the physical needs of institution

32. Direct the preparation of plans for new buildings and installments as needed

33. Plan for major repairs or alterations of plants, buildings and equipment

34. Recommend to board for equipment acquisitions

35. Ensure that physical plant master plans are current and followed as closely as possible

36. Ascertain that campus grounds are well-kept through appropriate officer and administrator

(Optional: One open-ended question here)
Actual Role Performance

(You Do)

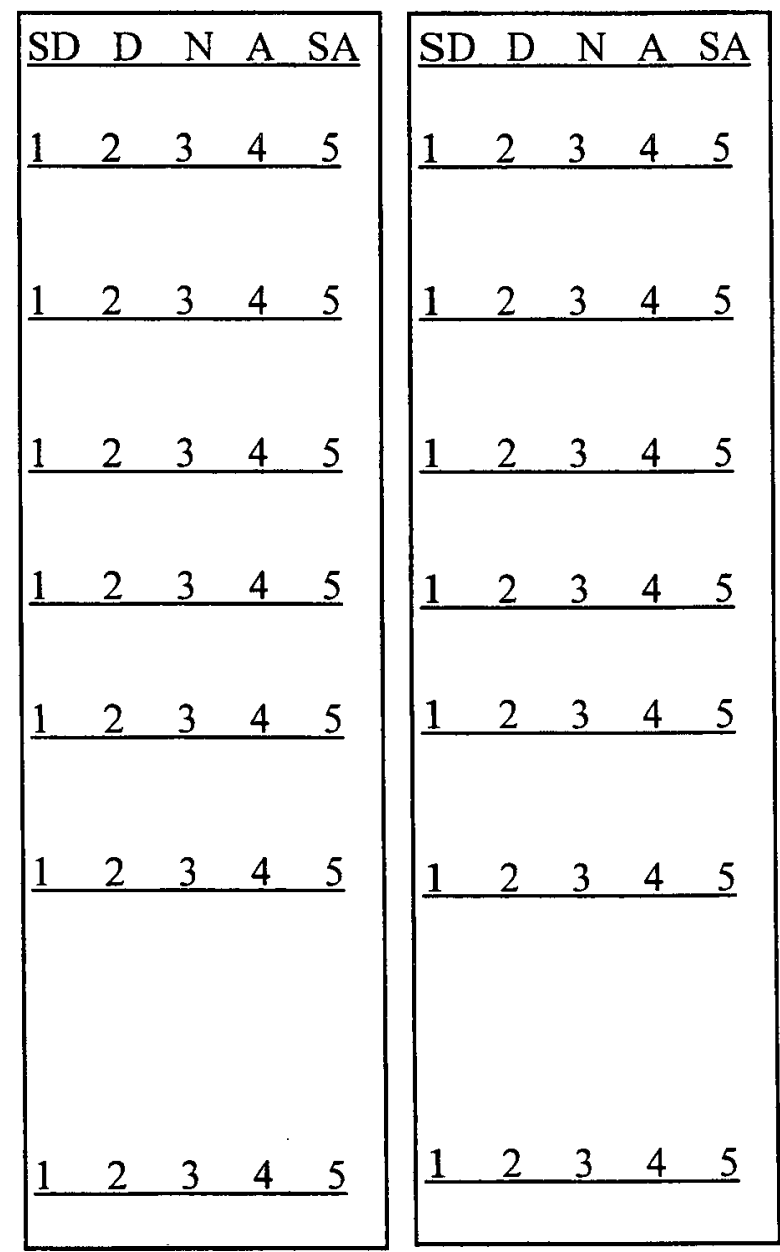




\section{COMMUNITY- AND ALUMNI-RELATED FUNCTIONS}

In this section you are asked about your perception of your present actual role performance and expected role responsibility in your community- and alumni-related functions as Seventh-day Adventist senior college/university president. Indicate your degree of agreement or disagreement with each statement by circling one number for each in both the grids. The scale: $1=$ you strongly disagree (SD), $2=$ you disagree (D), $3=$ you neither agree nor disagree $(\mathrm{N}), 4=$ you agree $(\mathrm{A})$, and $5=$ you strongly agree (SA).

\section{$\begin{array}{lllllll}\text { strongly disagree } & 1 & 2 & 3 & 4 & 5 & \text { strongly agree }\end{array}$}

As Seventh-day Adventist senior college/ university president you do and should

37. Assist in raising funds for support of institution

38. Represent institution to churches of the constituency

39. Present reports about institution to community and alumni

40. Represent institution as its official spokesperson to public

41. Associate closely with alumni groups

42. Listen to the constituency and alumni

Actual Role

Performance

(You Do)

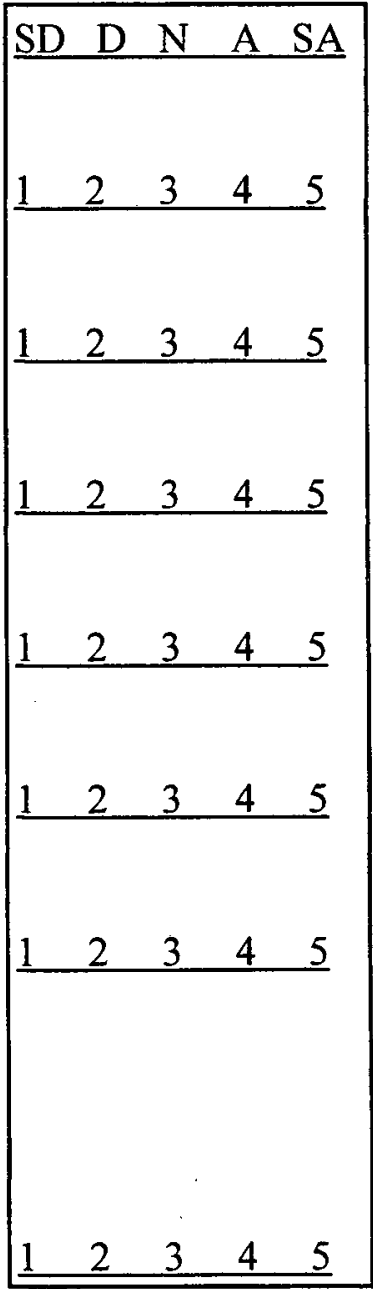

Expected Role

Responsibility

(You Should)

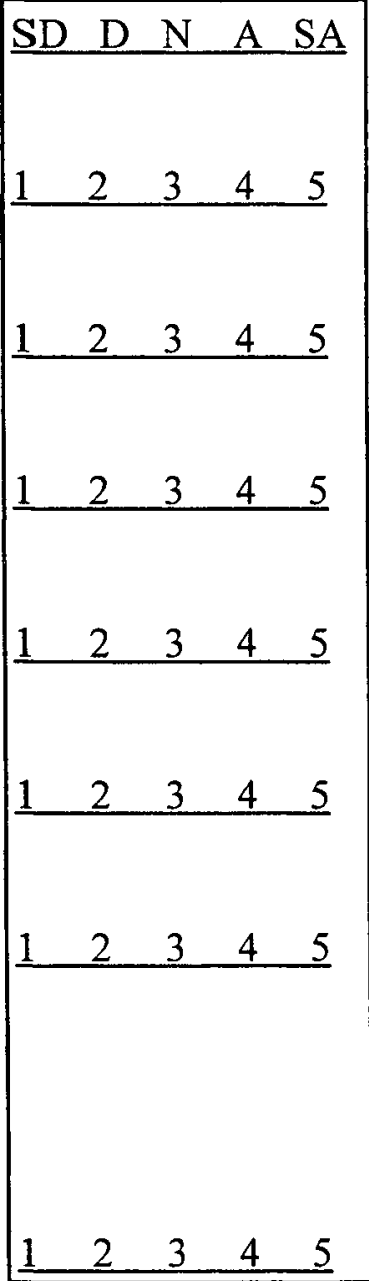

(Optional: One open-ended question here) 


\section{SPIRITUAL LIFE-RELATED FUNCTIONS}

In this section you are asked about your perception of your present actual role performance and expected role responsibility in your spiritual life-related functions as Seventh-day Adventist senior college/university president. Indicate your degree of agreement or disagreement with each statement by circling one number for each in both the grids. The scale: 1=you strongly disagree (SD), 2=you disagree (D), 3=you neither agree nor disagree (N), 4=you agree (A), and 5=you strongly agree (SA).

\section{$\begin{array}{llllllll}\text { strongly disagree } & 1 & 2 & 3 & 4 & 5 & \text { strongly agree }\end{array}$}

As Seventh-day Adventist senior college/ university president you do and should:

43. Assume primary responsibility for promotion of religious life of students at the institution via delegation

44. Assume primary responsibility for promotion of religious life of faculty and staff at the institution via delegation

45. Take ultimate responsibility for midweek chapels, vespers, weeks of spiritual emphasis and other religious programs of the institution via delegation for spiritual nurture

46. Manifest Christ-like character in spiritual leadership roles

47. Focus on Christian philosophy of institution

(Optional: One open-ended question here)
Actual Role

Performance

(You Do)

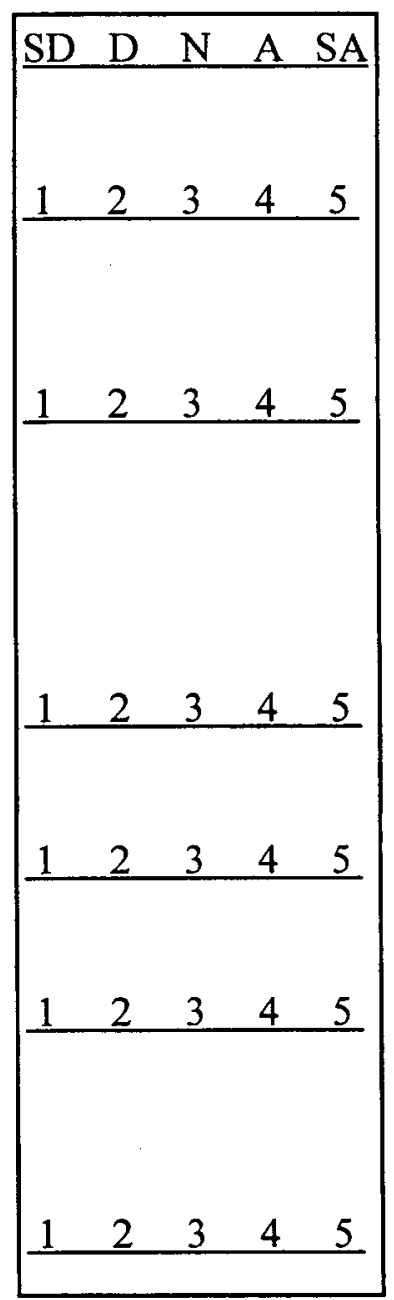

Expected Role

Responsibility

(You Should)

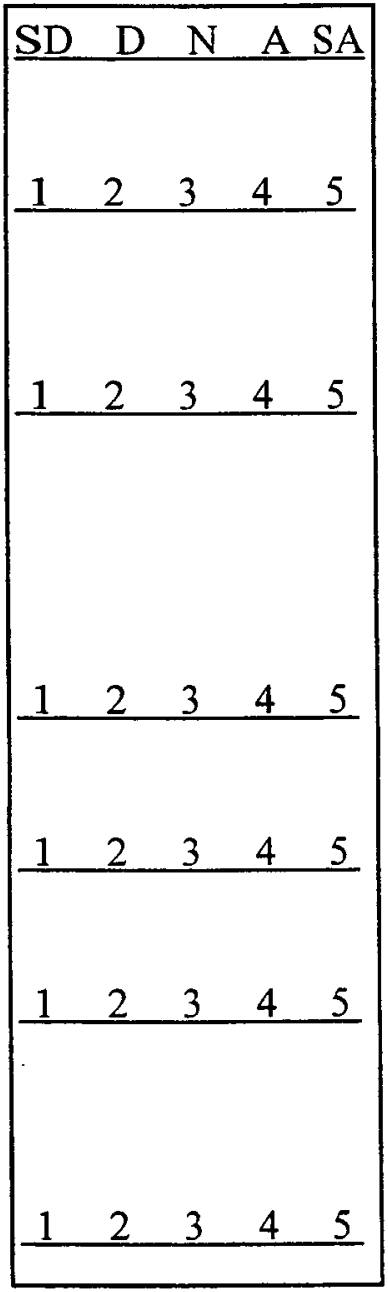


Questionnaire and Instructions for

Board of trustees' Chairs

Academic Vice Presidents

Student Service Vice Presidents

and Finance Vice Presidents 


\section{Role Performance and Role Expectation}

Of Seventh-day Adventist Senior College and University Presidents

As Perceived by Seventh-day Adventist Senior College/University's

Board of Trustees' Chairs, Academic Vice Presidents, Student Service Vice Presidents, and Finance Vice Presidents

Purpose of Questionnaire: The purpose of this questionnaire is to obtain data which will reveal the expectations and perceptions of college and university officials regarding the role and function of presidents of Seventh-day Adventist (SDA) universities and senior colleges. This study will investigate concerning levels of commons agreement and disagreement between respondent groups regarding actual and expected roles of SDA senior college and university presidents. The data will be used for a purpose beyond dissertation problem resolution. Your response will contribute significantly to the study of SDA college and university administration by clarifying the role and function of the president and his/her relationship with the supporting administrators.

Direction for Questionnaire: The questionnaire has 47 statements listed under 7 broad categories of functions with open slots for you to add any activity you feel should be included. Rank each activity on a continuum scale ranging from 1 (Strongly Disagree) to 5 (Strongly Agree). Circle the numeral that best reflects your view.

In the first grid, titled, Actual Role Performance, indicate the extent to which you feel your president is presently performing his/her functions. Rank each of these perceptions on the scale following the activity by assigning it a rating of 1 , if you know the president does not perform this function, and 5 , if you know that the president demonstrates significant responsibility for its performance. Assign values of 2,3, or 4 for intermediate responsibility for performance. Circle only one numeral for each activity which indicates your perception for your college/university president's present actual role performance.

In the second grid, titled, Expected Role Responsibility, indicate the extent to which you expect that the item of function should belong to the president's area of responsibility, or you expect him/her to perform it in the capacity of a president. Rank each of these activities on the scale following the activity by assigning it a rating of 1 , if you do not expect your president to perform these functions, and 5 , if you expect very highly that your president should perform these responsibilities. Assign values of 2, 3, or 4 for intermediate degrees of your expectations. Circle only one numeral for each activity which you feel applies.

Please respond to all items of the seven sections in the two grids. Anonymity and confidentiality will be maintained. Do not write your or your institution's name anywhere. After you have completed the questionnaire, please place it in the enclosed self-addressed postage stamped envelope and mail it today. If you have any question, please contact the researcher or the research advisor. Thank you for participating in this research project.

Researcher: Sunith K. Das, 4775 Kimber Lane, Berrien Springs, MI 49103. Phone: (269) 473-1612. Email: <dass@andrews.edu $\geq$

Dissertation Committee Chair: Hinsdale Bernard, Ph.D., Professor of Education, Andrews University, Berrien Springs, MI 49104. Phone (269) 471-6702. E-mail:<hbernard@andrews.edu> 


\section{STUDENT-RELATED FUNCTIONS}

In this section you are asked about your perception of Seventh-day Adventist senior college/university president's student-related functions as he or she is actually performing and as you expect him or her to perform in your institution. Indicate your degree of agreement or disagreement with each statement by circling one number for each in both the grids. The scale: $1=$ you strongly disagree (SD), $2=$ you disagree (D), $3=$ you neither agree nor disagree $(\mathrm{N}), 4=$ you agree $(\mathrm{A})$, and $5=$ you strongly agree (SA).

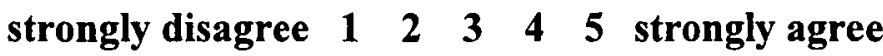

The Seventh-day Adventist senior college/ university president does and should:

1. Participate in student recruitment

2. Work closely with student government leaders

3. Dialogue frequently with students

4. Visit students periodically in the dorms during dorm worships

5. Take ultimate responsibility for student discipline

6. Teach some classes in order to maintain contact with students

7. Know every student personally

8. Serve as mentor of students (Optional: One open-ended question here)
Actual Role

Performance

(Does)

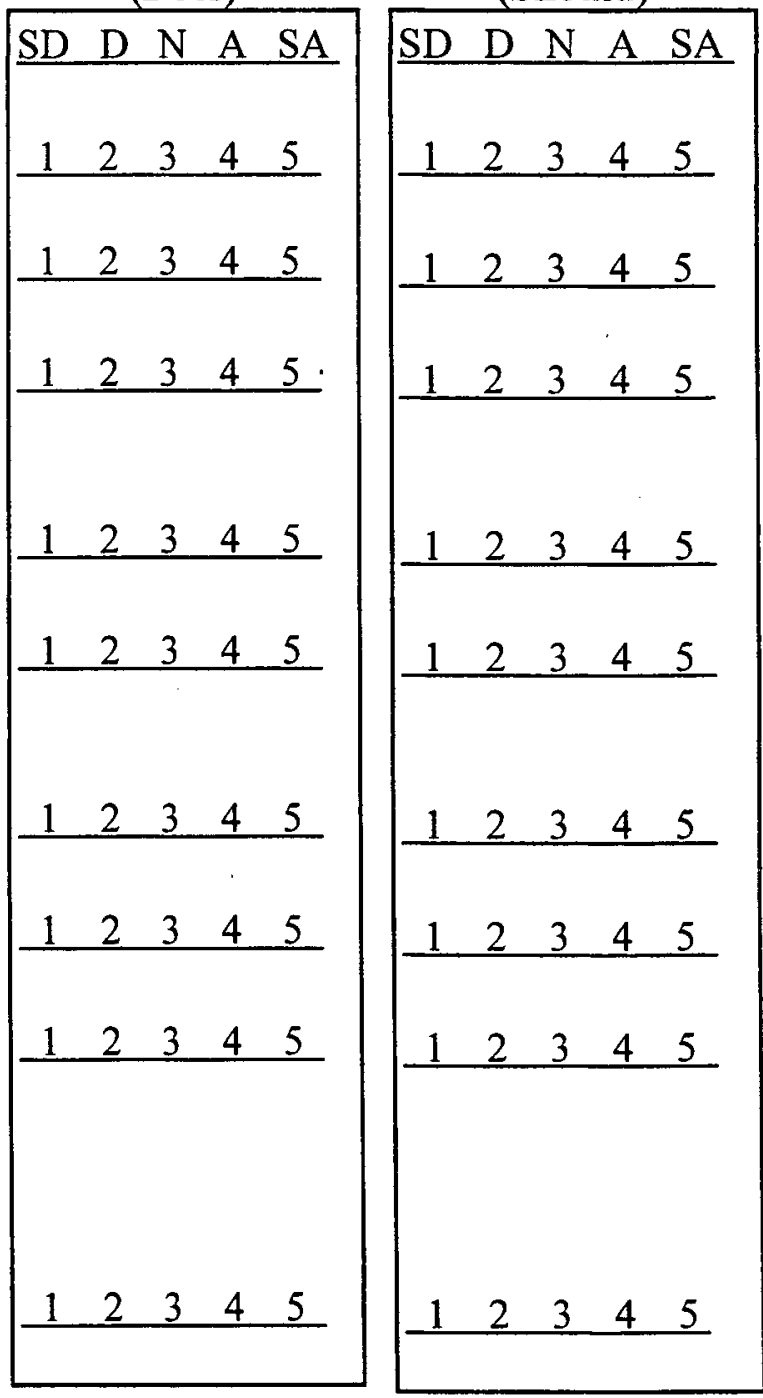




\section{EMPLOYEE-RELATED FUNCTIONS}

In this section you are asked about your own perception of Seventh-day Adventist senior college/university president's employee-related functions as he or she is actually performing and as you expect him or her to perform in your institution. Indicate your degree of agreement or disagreement with each statement by circling one number for each in both the grids. The scale: $1=$ you strongly disagree (SD), $2=$ you disagree (D), $3=$ you neither agree nor disagree $(\mathrm{N}), 4=$ you agree $(\mathrm{A})$, and $5=$ you strongly agree (SA).

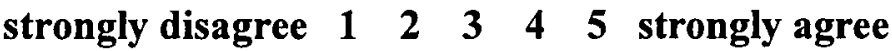

The Seventh-day Adventist senior college/ university president does and should:

9. Participate in faculty recruitment

10. Serve as academic leader of faculty

11. Guard faculty's academic freedom

12. Have responsibility in submitting proposed changes in academic matters to faculty for approval

13. Work with faculty in reviewing accrediting agency's reports

14. Recommend to board for hiring, promotion, demotion, and dismissal of faculty and staff

15. Collaborate for advanced inservice training for faculty \& staff

16. Carry out policies and regulations of the faculty and staff

(Optional: One open-ended question here).
Actual Role

Performance

(Does)

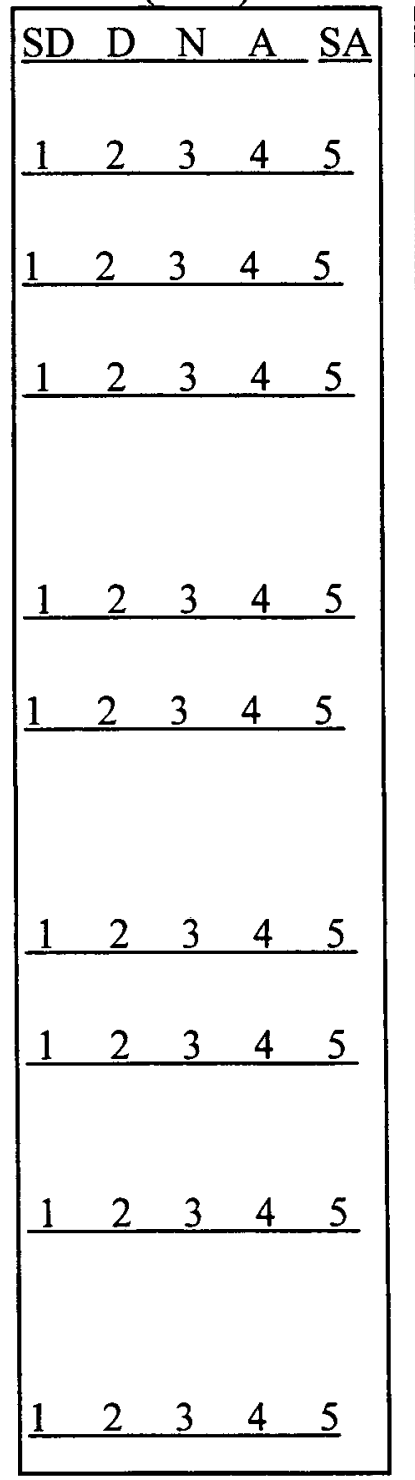

Expected Role

Responsibility

(Should)

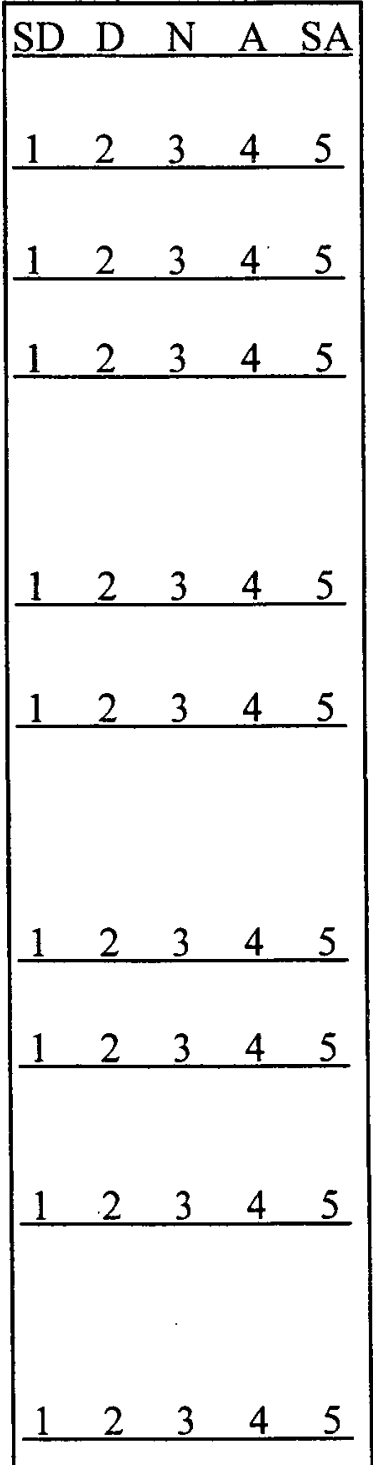




\section{GENERAL ADMINISTRATIVE FUNCTIONS}

In this section you are asked about your own perception of Seventh-day Adventist senior college/university president's general administrative functions as he or she is actually performing and as you expect him/her to perform in your institution. Indicate your degree of agreement or disagreement with each statement by circling one number for each in both the grids. The scale: $1=$ you strongly disagree (SD), $2=$ you disagree (D), $3=$ you neither agree nor disagree $(\mathrm{N}), 4=$ you agree $(\mathrm{A})$, and $5=$ you strongly agree $(\mathrm{SA})$.

\section{$\begin{array}{lllllll}\text { strongly disagree } & 1 & 2 & 3 & 4 & 5 & \text { strongly agree }\end{array}$}

The Seventh-day Adventist senior college/ university president does and should:

17. Prepare agenda for board of trustees' meetings

18. Preside at general faculty meetings

19. Take responsibility in structuring student admission standards

20. Take responsibility in strategic planning for institution

21. Take a leading role to prepare, revise and follow organizational chart

22. Foster research and intellectual climate for institution

23. Comply with legal mandates and laws in addressing grievances

24. Help formulate and adhere to institution's educational philosophy

25. Delegate responsibility, authority, and needed resources to appropriate administrators and support their roles (Optional: One open-ended question here)
Actual Role

Performance

(Does)

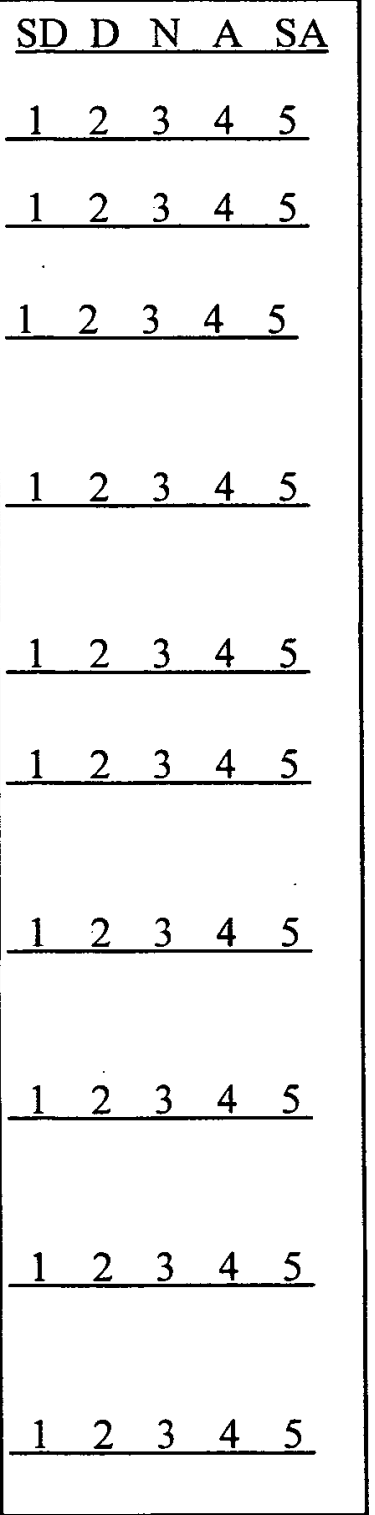

Expected Role

Responsibility

(Should)

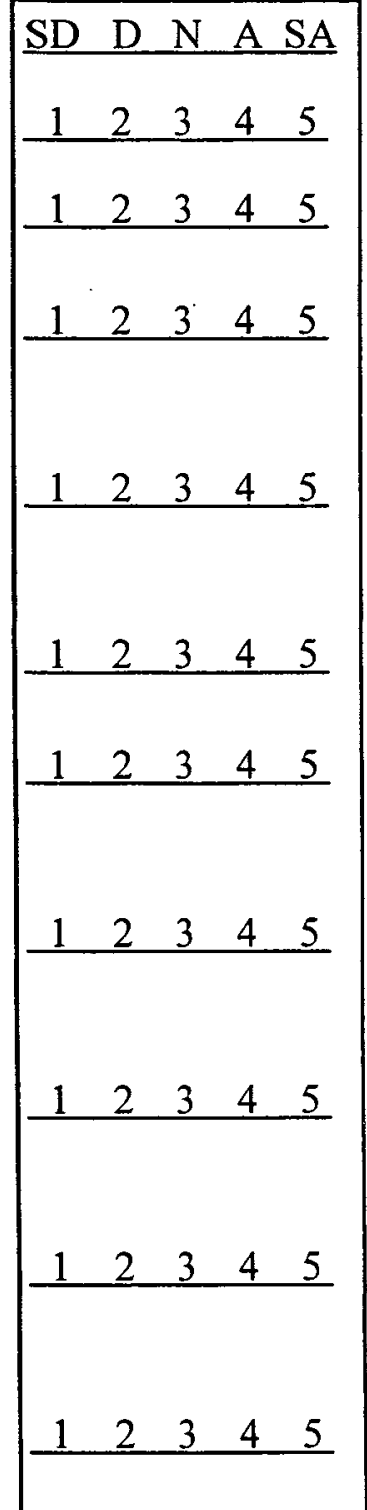




\section{FINANCE-RELATED FUNCTIONS}

In this section you are asked about your own perception of Seventh-day Adventist senior college/university president's finance-related functions as he or she is actually performing and as you expect him or her to perform in your institution. Indicate your degree of agreement or disagreement with each statement by circling one number for each in both the grids. The scale: $1=$ you strongly disagree (SD), $2=$ you disagree (D), $3=$ you neither agree nor disagree $(\mathrm{N}), 4=$ you agree $(\mathrm{A})$, and $5=$ you strongly agree $(\mathrm{SA})$.

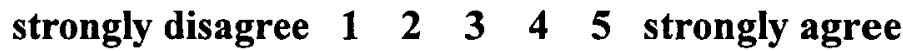

Actual Role

Performance

(Does)

The Seventh-day Adventist senior college/ university president does and should:

26. Participate in the preparation of annual budget of the institution

27. Ensure that institution's budget, after approval by board, is adhered to

28. Present institution's annual audited financial statements to board of trustees

29. Recommend to board the salary changes for faculty/staff positions

30. Accept accountability to board for financial operation of institution

(Optional: One open-ended question here)

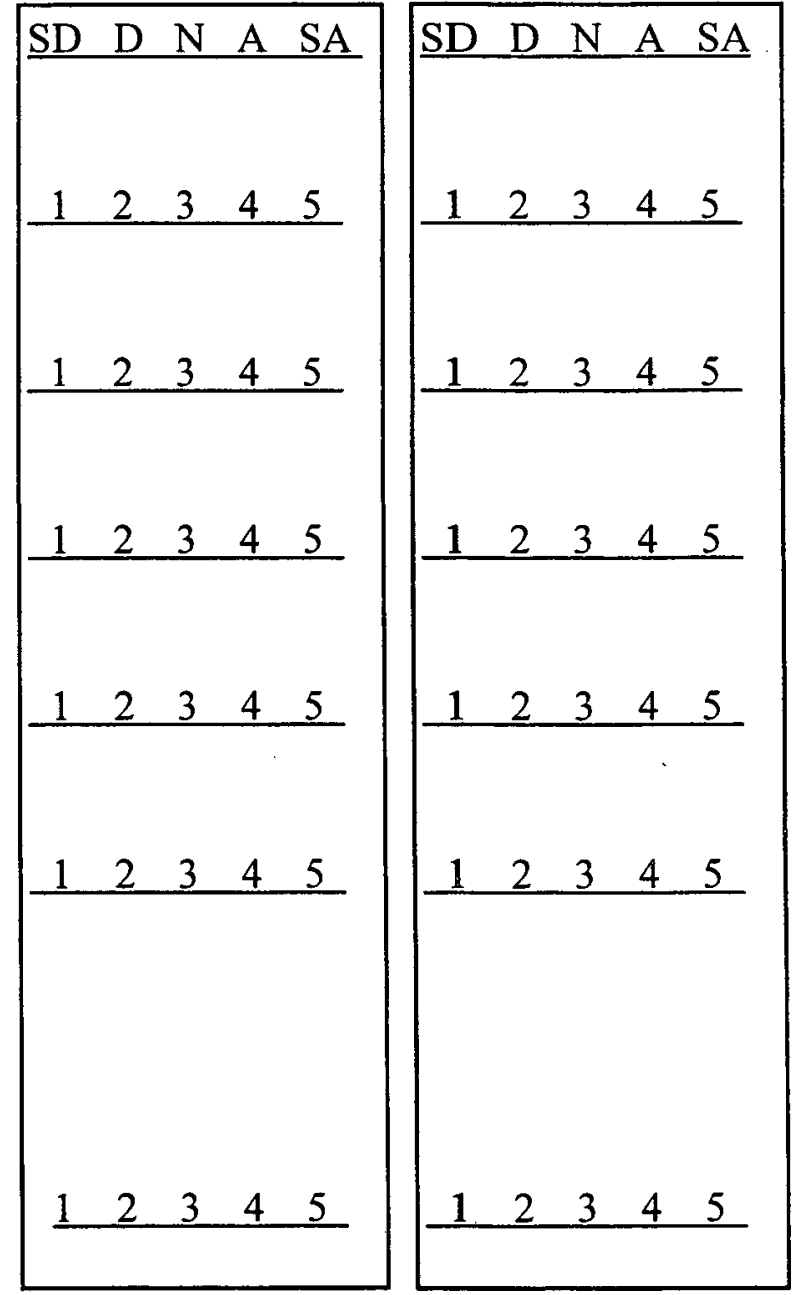




\section{PHYSICAL FACILITIES-RELATED FUNCTIONS}

In this section you are asked about your own perception of Seventh-day Adventist senior college/university president's physical facilities-related functions as he or she is actually performing and as you expect him or her to perform in your institution. Indicate your degree of agreement or disagreement with each statement by circling one number for each in both the grids. The scale: $1=$ you strongly disagree (SD), $2=$ you disagree (D), $3=$ you neither agree nor disagree $(\mathrm{N}), 4=$ you agree $(\mathrm{A})$, and $5=$ you strongly agree (SA).

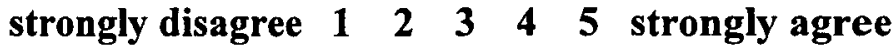

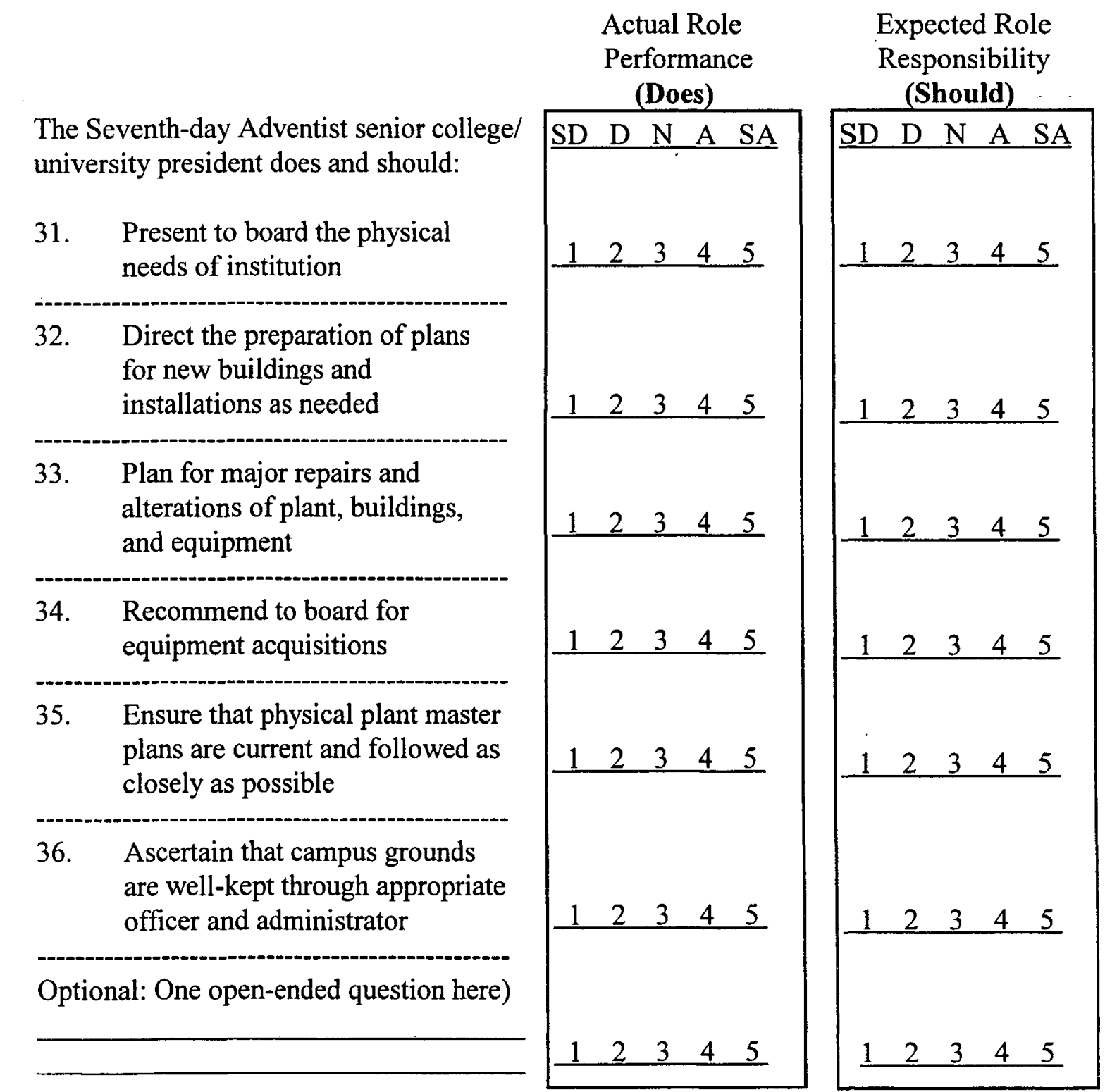




\section{COMMUNITY- AND ALUMNI-RELATED FUNCTIONS}

In this section you are asked about your perception of Seventh-day Adventist senior college/university president's community and alumni-related functions as he or she is actually performing and as you expect him or her to perform in your institution. Indicate your degree of agreement or disagreement with each statement by circling one number for each in both the grids. The scale: $1=$ you strongly disagree (SD), $2=$ you disagree (D), $3=$ you neither agree nor disagree $(\mathrm{N}), 4=$ you agree $(\mathrm{A})$, and $5=$ you stron gly agree (SA).

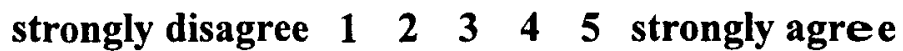

The Seventh-day Adventist senior college/ university president does and should:

37. Assist in raising funds for support of institution

38. Represent institution to churches of the constituency

39. Present reports about institution to community and alumni

40. Represent institution as its official spokesperson to public

41. Associate closely with alumni groups

42. Listen to constituency and alumni

(Optional: One open-ended question here)
Actual Role

Performance

(Does)

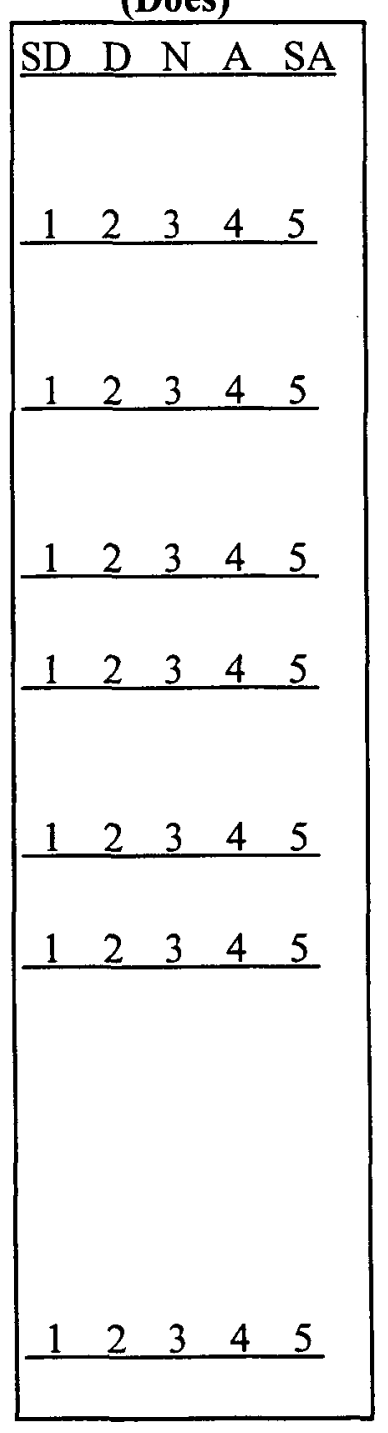

Expected Role

Responsibility

(Should)

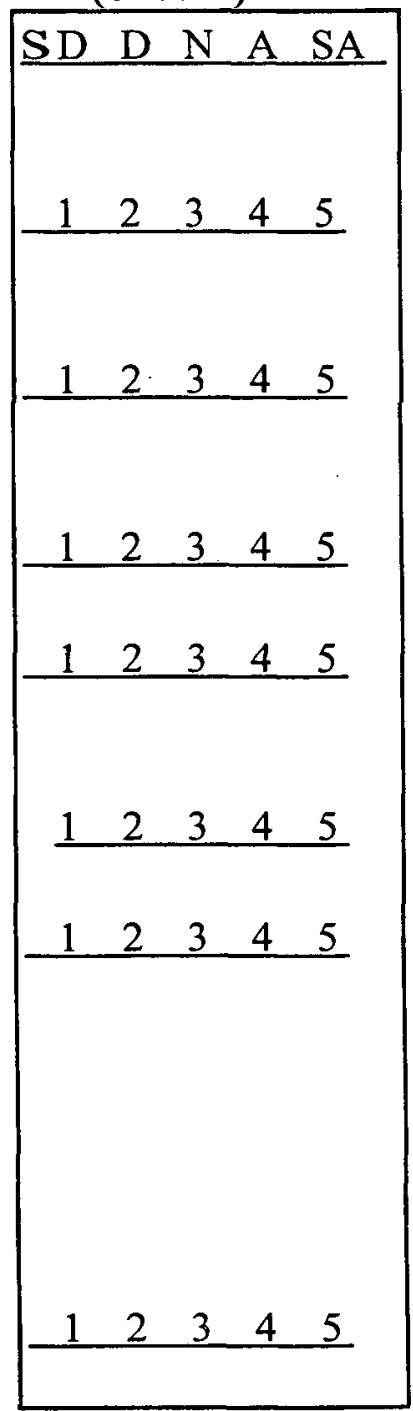




\section{SPIRITUAL LIFE-RELATED FUNCTIONS}

In this section you are asked about your own perception of Seventh-day Adventist senior college/university president's spiritual life-related functions as he or she is actually performing and as you expect him or her to perform in your institution. Indicate your degree of agreement or disagreement with each statement by circling one number for each in both the grids. $1=$ you strongly disagree (SD), $2=$ you disagree (D), $3=$ you neither agree nor disagree $(\mathrm{N}), 4=$ you agree $(\mathrm{A})$, and $5=$ you strongly agree $(\mathrm{SA})$.

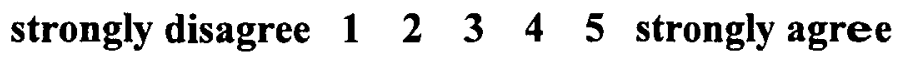

The Seventh-day Adventist senior college/ university president does and should:

43. Assume primary responsibility for promotion of religious life of students at the institution via delegation

44. Assume primary responsibility for promotion of religious life of faculty and staff at the institution via delegation

45. Take ultimate responsibility for midweek chapels, vespers, weeks of spiritual emphasis, and other religious programs of the institution via delegation for spiritual nurture

46. Manifest Christ-like character in spiritual leadership roles

47. Focus on Christian philosophy of institution

(Optional: One open-ended question here)
Actual Role

Performance

(Does)

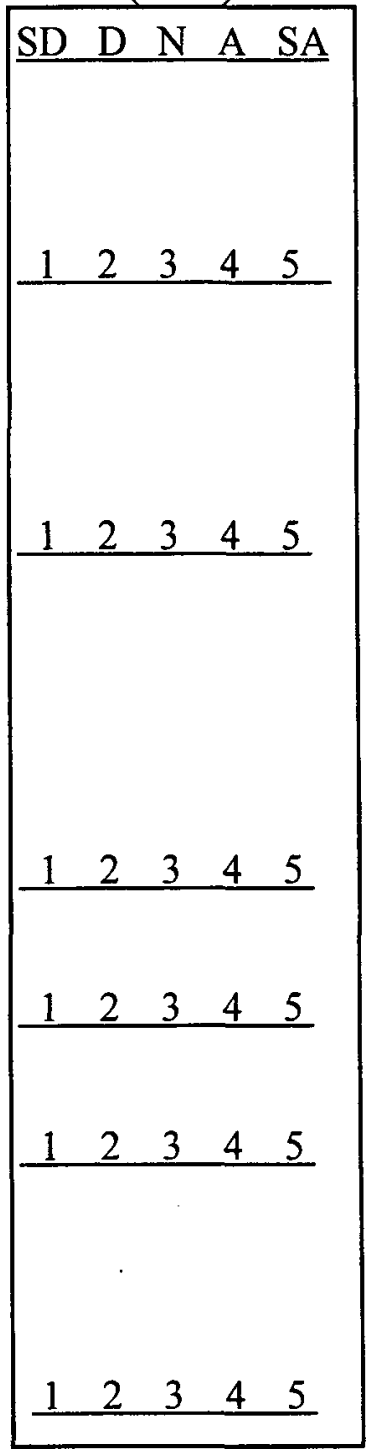

Expected Role Responsibility

(Should)

\begin{tabular}{lllll}
\hline SD & D & $N$ & A & SA \\
\hline
\end{tabular}
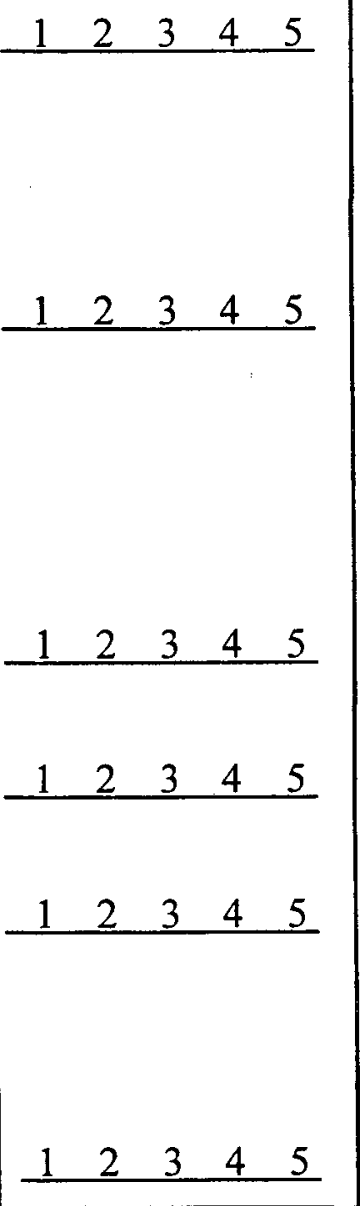
APPENDIX G

TABLES 33-39

COMPOSITE ANOVA RESULTS FOR ACTUAL ROLES

SHOWING MEANS AND STANDARD DEVIATIONS

FOR SUB-HYPOTHESES 1-47 
Table 33. Composite ANOVA table for sub-hypotheses 1-8, showing means and standard deviations of subgroups for student-related functions--actual roles

\begin{tabular}{|c|c|c|c|c|c|c|c|c|}
\hline Sub-Hypothesis & $\begin{array}{l}\text { Board } \\
\text { Chair }\end{array}$ & $\begin{array}{c}\text { Presi- } \\
\text { dent }\end{array}$ & $\begin{array}{l}\text { Aca- } \\
\text { demic } \\
\text { VP }\end{array}$ & $\begin{array}{c}\text { Student } \\
\text { Service } \\
\text { VP }\end{array}$ & $\begin{array}{c}\text { Finance } \\
\text { VP }\end{array}$ & Total & $F$ & $P$ \\
\hline $\begin{array}{l}\text { 1. Participate in student } \\
\text { recruitment } \\
M \\
S D \\
N\end{array}$ & $\begin{array}{r}3.50 \\
1.50 \\
18\end{array}$ & $\begin{array}{r}3.65 \\
1.17 \\
17\end{array}$ & $\begin{array}{r}2.62 \\
1.15 \\
16\end{array}$ & $\begin{array}{r}3.46 \\
1.45 \\
13\end{array}$ & $\begin{array}{r}3.36 \\
1.34 \\
14\end{array}$ & $\begin{array}{r}3.32 \\
1.26 \\
78\end{array}$ & 1.688 & .162 \\
\hline $\begin{array}{l}\text { 2. Work closely with } \\
\text { student government } \\
\text { leaders } \\
M \\
S D \\
N\end{array}$ & $\begin{array}{c}3.61 \\
.92 \\
18\end{array}$ & $\begin{array}{c}3.59 \\
1.23 \\
17 \\
\end{array}$ & $\begin{array}{r}3.06 \\
1.18 \\
16\end{array}$ & $\begin{array}{r}3.15 \\
1.46 \\
13\end{array}$ & $\begin{array}{r}2.93 \\
1.38 \\
14\end{array}$ & $\begin{array}{r}3.29 \\
1.23 \\
78\end{array}$ & 1.040 & .393 \\
\hline $\begin{array}{l}\text { 3. Dialogue frequently } \\
\text { with students } \\
\qquad \begin{array}{c}M \\
S D \\
N\end{array}\end{array}$ & $\begin{array}{r}3.72 \\
.96 \\
18\end{array}$ & $\begin{array}{r}4.23 \\
.75 \\
17\end{array}$ & $\begin{array}{r}3.25 \\
1.29 \\
16\end{array}$ & $\begin{array}{r}3.54 \\
1.20 \\
13\end{array}$ & $\begin{array}{r}3.86 \\
1.35 \\
14\end{array}$ & $\begin{array}{r}3.73 \\
1.14 \\
78\end{array}$ & 1.758 & .147 \\
\hline $\begin{array}{l}\text { 4. Visit students } \\
\text { periodically in the } \\
\text { dorms during their } \\
\text { dorm worships } \\
M \\
S D \\
N\end{array}$ & $\begin{array}{r}2.78 \\
1.17 \\
18\end{array}$ & $\begin{array}{r}2.89 \\
1.32 \\
17\end{array}$ & $\begin{array}{r}2.13 \\
1.26 \\
16\end{array}$ & $\begin{array}{r}2.15 \\
1.07 \\
13\end{array}$ & $\begin{array}{r}2.71 \\
1.33 \\
14\end{array}$ & $\begin{array}{r}2.55 \\
1.24 \\
78\end{array}$ & 1.333 & .266 \\
\hline $\begin{array}{l}\text { 5. Take ultimate } \\
\text { responsibility for } \\
\text { student discipline } \\
M \\
S D \\
N\end{array}$ & $\begin{array}{c}3.50 \\
1.29 \\
18\end{array}$ & $\begin{array}{r}3.00 \\
1.58 \\
17\end{array}$ & $\begin{array}{r}2.19 \\
1.11 \\
16\end{array}$ & $\begin{array}{r}3.00 \\
1.68 \\
13\end{array}$ & $\begin{array}{r}2.86 \\
1.41 \\
14\end{array}$ & $\begin{array}{r}2.92 \\
1.45 \\
78\end{array}$ & 1.853 & .128 \\
\hline $\begin{array}{l}\text { 6. Teach some classes } \\
\text { to maintain contact } \\
\text { with students } \\
M \\
S D \\
N\end{array}$ & $\begin{array}{r}2.56 \\
1.69 \\
18\end{array}$ & $\begin{array}{r}2.94 \\
1.56 \\
17\end{array}$ & $\begin{array}{r}1.75 \\
1.24 \\
16\end{array}$ & $\begin{array}{r}2.00 \\
1.29 \\
13\end{array}$ & $\begin{array}{r}2.93 \\
1.69 \\
14\end{array}$ & $\begin{array}{r}2.45 \\
1.55 \\
78\end{array}$ & 1.961 & .109 \\
\hline
\end{tabular}


Table 33-Continued.

\begin{tabular}{|c|r|r|r|r|r|r|r|r|}
\hline Sub-Hypotheses & $\begin{array}{c}\text { Board } \\
\text { Chair }\end{array}$ & $\begin{array}{c}\text { Presi- } \\
\text { dent }\end{array}$ & $\begin{array}{c}\text { Aca- } \\
\text { demic } \\
\text { VP }\end{array}$ & $\begin{array}{c}\text { Student } \\
\text { Service } \\
\text { VP }\end{array}$ & $\begin{array}{c}\text { Finance } \\
\text { VP }\end{array}$ & Total & $F$ & $P$ \\
\hline 7. Know every student & & & & & & & & \\
personally & & & & & & & & \\
$M$ & 2.39 & 2.00 & 1.75 & 2.46 & 1.93 & 2.10 & 1.013 & .406 \\
$S D$ & 1.50 & .94 & .93 & 1.45 & 1.00 & 1.19 & & \\
$N$ & 18 & 17 & 16 & 13 & 14 & 78 & & \\
\hline 8. Serve as mentor of & & & & & & & & \\
students & & & & & & & & \\
$M$ & 3.33 & 3.12 & 2.00 & 3.15 & 2.93 & 2.91 & 3.085 & .021 \\
$S D$ & 1.08 & 1.32 & .73 & 1.52 & 1.33 & 1.27 & & \\
$N$ & 18 & 17 & 16 & 13 & 14 & 78 & & \\
\hline
\end{tabular}


Table 34. Composite ANOVA table for sub-hypotheses 9-16, showing means and standard deviations of subgroups for employee-related functions--actual roles

\begin{tabular}{|c|c|c|c|c|c|c|c|c|}
\hline Sub-Hypotheses & $\begin{array}{l}\text { Board } \\
\text { Chair }\end{array}$ & $\begin{array}{c}\text { Presi- } \\
\text { dent }\end{array}$ & $\begin{array}{l}\text { Aca- } \\
\text { demic } \\
\text { VP }\end{array}$ & $\begin{array}{c}\text { Student } \\
\text { Service } \\
\text { VP }\end{array}$ & $\begin{array}{c}\text { Finance } \\
\text { VP }\end{array}$ & Total & $F$ & $P$ \\
\hline $\begin{array}{l}\text { 9. Participate in faculty } \\
\text { recruitment } \\
M \\
S D \\
N\end{array}$ & $\begin{array}{r}4.39 \\
.70 \\
18\end{array}$ & $\begin{array}{r}4.24 \\
.90 \\
17\end{array}$ & $\begin{array}{r}3.50 \\
.97 \\
16\end{array}$ & $\begin{array}{r}3.92 \\
1.12 \\
13\end{array}$ & $\begin{array}{r}4.14 \\
1.17 \\
14\end{array}$ & $\begin{array}{r}4.05 \\
.99 \\
78\end{array}$ & 2.100 & .090 \\
\hline $\begin{array}{l}\text { 10. Serve as academic } \\
\text { leader of faculty } \\
M \\
S D \\
N\end{array}$ & $\begin{array}{r}3.22 \\
1.48 \\
18\end{array}$ & $\begin{array}{r}3.12 \\
1.27 \\
17\end{array}$ & $\begin{array}{r}2.57 \\
1.03 \\
16\end{array}$ & $\begin{array}{r}2.69 \\
1.38 \\
13\end{array}$ & $\begin{array}{r}3.21 \\
1.05 \\
14\end{array}$ & $\begin{array}{r}2.97 \\
1.26 \\
78\end{array}$ & .946 & .443 \\
\hline $\begin{array}{l}\text { 11. Guard faculty's } \\
\text { academic freedom } \\
M \\
S D \\
N\end{array}$ & $\begin{array}{r}3.89 \\
.83 \\
18\end{array}$ & $\begin{array}{r}4.06 \\
1.20 \\
17\end{array}$ & $\begin{array}{r}3.31 \\
1.14 \\
16\end{array}$ & $\begin{array}{r}3.31 \\
.95 \\
13\end{array}$ & $\begin{array}{r}3.93 \\
.92 \\
14\end{array}$ & $\begin{array}{r}3.72 \\
1.04 \\
78\end{array}$ & 1.910 & .118 \\
\hline $\begin{array}{l}\text { 12. Have responsibility } \\
\text { in submitting } \\
\text { proposed changes in } \\
\text { academic matters to } \\
\text { faculty for approval } \\
M \\
S D \\
N\end{array}$ & $\begin{array}{r}3.56 \\
1.15 \\
18\end{array}$ & $\begin{array}{r}2.47 \\
1.37 \\
17\end{array}$ & $\begin{array}{r}2.31 \\
1.20 \\
16\end{array}$ & $\begin{array}{r}3.31 \\
1.38 \\
13\end{array}$ & $\begin{array}{r}3.21 \\
1.12 \\
14\end{array}$ & $\begin{array}{r}2.96 \\
1.31 \\
78\end{array}$ & 3.165 & .019 \\
\hline $\begin{array}{l}\text { 13. Work with faculty in } \\
\text { reviewing accrediting } \\
\text { agency's reports } \\
M \\
S D \\
N\end{array}$ & $\begin{array}{r}4.06 \\
.87 \\
18\end{array}$ & $\begin{array}{r}4.00 \\
1.06 \\
17\end{array}$ & $\begin{array}{r}2.94 \\
1.29 \\
16\end{array}$ & $\begin{array}{r}3.85 \\
.80 \\
13\end{array}$ & $\begin{array}{r}3.79 \\
1.37 \\
14\end{array}$ & $\begin{array}{r}3.73 \\
1.15 \\
78\end{array}$ & 2.784 & .033 \\
\hline $\begin{array}{l}\text { 14. Recommend to board } \\
\text { for hiring, promotion, } \\
\text { demotion and } \\
\text { dismissal of faculty } \\
\text { and staff } \\
M \\
S D \\
N\end{array}$ & $\begin{array}{r}3.83 \\
1.25 \\
18\end{array}$ & $\begin{array}{r}4.29 \\
1.26 \\
17\end{array}$ & $\begin{array}{r}4.06 \\
1.34 \\
16\end{array}$ & $\begin{array}{r}4.46 \\
.97 \\
13\end{array}$ & $\begin{array}{r}4.21 \\
.70 \\
14 \\
\end{array}$ & $\begin{array}{r}4.15 \\
1.14 \\
78 \\
\end{array}$ & .680 & .608 \\
\hline
\end{tabular}


Table 34--Continued.

\begin{tabular}{|c|c|c|c|c|c|c|c|c|}
\hline Sub-Hypotheses & $\begin{array}{l}\text { Board } \\
\text { Chair }\end{array}$ & $\begin{array}{c}\text { Presi- } \\
\text { dent }\end{array}$ & $\begin{array}{l}\text { Aca- } \\
\text { demic } \\
\text { VP }\end{array}$ & $\begin{array}{l}\text { Student } \\
\text { Service } \\
\text { VP }\end{array}$ & $\begin{array}{l}\text { Finance } \\
\text { VP }\end{array}$ & Total & $F$ & $P$ \\
\hline \multicolumn{9}{|l|}{$\begin{array}{l}\text { 15. Collaborate for } \\
\text { advanced in-service } \\
\text { training for faculty } \\
\text { and staff }\end{array}$} \\
\hline$M$ & 3.67 & 3.82 & 2.63 & 3.85 & 3.14 & 3.42 & 3.066 & .022 \\
\hline$S D$ & 1.14 & 1.01 & 1.45 & 1.21 & 1.10 & 1.25 & & \\
\hline$N$ & 18 & 17 & 16 & 13 & 14 & 78 & & \\
\hline \multicolumn{9}{|l|}{$\begin{array}{l}\text { 16. Carry out policies and } \\
\text { regulations of faculty } \\
\text { and staff }\end{array}$} \\
\hline$M$ & 3.78 & 4.06 & 3.25 & 3.54 & 3.36 & 3.62 & .967 & .431 \\
\hline$S D$ & 1.17 & 1.30 & 1.61 & 1.39 & 1.22 & 1.34 & & \\
\hline$N$ & 18 & 17 & 16 & 13 & 14 & 78 & & \\
\hline
\end{tabular}


Table 35. Composite ANOVA table for sub-hypotheses 17-25, showing means and standard deviations of subgroups for general administrative functions--actual roles

\begin{tabular}{|c|c|c|c|c|c|c|c|c|}
\hline Sub-Hypotheses & $\begin{array}{l}\text { Board } \\
\text { Chair }\end{array}$ & $\begin{array}{c}\text { Presi- } \\
\text { dent }\end{array}$ & $\begin{array}{l}\text { Aca- } \\
\text { demic } \\
\text { VP }\end{array}$ & $\begin{array}{l}\text { Student } \\
\text { Service } \\
\text { VP }\end{array}$ & $\begin{array}{c}\text { Finance } \\
\text { VP }\end{array}$ & Total & $F$ & $P$ \\
\hline $\begin{array}{l}\text { 17. Prepare agenda for } \\
\text { board of trustees' } \\
\text { meetings } \\
M \\
S D \\
N\end{array}$ & $\begin{array}{r}4.33 \\
1.08 \\
18\end{array}$ & $\begin{array}{r}5.00 \\
.00 \\
17\end{array}$ & $\begin{array}{r}4.75 \\
.68 \\
16\end{array}$ & $\begin{array}{r}4.54 \\
.97 \\
13\end{array}$ & $\begin{array}{r}4.57 \\
.65 \\
14\end{array}$ & $\begin{array}{r}4.64 \\
.79 \\
78\end{array}$ & 1.793 & .139 \\
\hline $\begin{array}{l}\text { 18. Preside over general } \\
\text { faculty meetings } \\
M \\
S D \\
N\end{array}$ & $\begin{array}{r}4.50 \\
.86 \\
18\end{array}$ & $\begin{array}{r}4.12 \\
1.36 \\
17\end{array}$ & $\begin{array}{r}4.06 \\
1.29 \\
16\end{array}$ & $\begin{array}{r}4.46 \\
1.51 \\
13\end{array}$ & $\begin{array}{r}3.57 \\
1.50 \\
14\end{array}$ & $\begin{array}{r}3.99 \\
1.32 \\
78\end{array}$ & 1.639 & .174 \\
\hline $\begin{array}{l}\text { 19. Take responsibility in } \\
\text { structuring student } \\
\text { admission standards } \\
M \\
S D \\
N\end{array}$ & $\begin{array}{r}3.67 \\
.77 \\
18\end{array}$ & $\begin{array}{r}2.71 \\
1.45 \\
17\end{array}$ & $\begin{array}{r}2.44 \\
1.15 \\
16\end{array}$ & $\begin{array}{r}2.85 \\
1.41 \\
13\end{array}$ & $\begin{array}{r}3.14 \\
.86 \\
14\end{array}$ & $\begin{array}{r}2.97 \\
1.21 \\
78\end{array}$ & 2.840 & .030 \\
\hline $\begin{array}{l}\text { 20. Take responsibility in } \\
\text { strategic planning for } \\
\text { the institution } \\
M \\
S D \\
N\end{array}$ & $\begin{array}{r}4.44 \\
.86 \\
18\end{array}$ & $\begin{array}{r}4.47 \\
.80 \\
17\end{array}$ & $\begin{array}{r}3.94 \\
1.39 \\
16\end{array}$ & $\begin{array}{r}4.54 \\
.97 \\
13\end{array}$ & $\begin{array}{r}4.07 \\
1.14 \\
14\end{array}$ & $\begin{array}{r}4.29 \\
1.05 \\
78\end{array}$ & 1.016 & .405 \\
\hline $\begin{array}{l}\text { 21. Take a leading role to } \\
\text { prepare, revise and } \\
\text { follow organizational } \\
\text { chart } \\
M \\
S D \\
N\end{array}$ & $\begin{array}{r}4.28 \\
.83 \\
18\end{array}$ & $\begin{array}{r}4.35 \\
1.11 \\
17\end{array}$ & $\begin{array}{r}3.50 \\
1.37 \\
16\end{array}$ & $\begin{array}{r}4.15 \\
.99 \\
13\end{array}$ & $\begin{array}{r}3.86 \\
1.10 \\
14\end{array}$ & $\begin{array}{r}4.04 \\
1.11 \\
78\end{array}$ & 1.677 & .167 \\
\hline $\begin{array}{l}\text { 22. Foster research and } \\
\text { intellectual climate } \\
\text { for the institution } \\
M \\
S D \\
N\end{array}$ & $\begin{array}{r}3.94 \\
.94 \\
18\end{array}$ & $\begin{array}{r}4.00 \\
1.06 \\
17\end{array}$ & $\begin{array}{r}3.38 \\
1.31 \\
16\end{array}$ & $\begin{array}{r}3.69 \\
1.38 \\
13\end{array}$ & $\begin{array}{r}4.00 \\
.96 \\
14\end{array}$ & $\begin{array}{r}3.81 \\
1.13 \\
78\end{array}$ & .908 & .464 \\
\hline
\end{tabular}


Table 35--Continued.

\begin{tabular}{|c|c|c|c|c|c|c|c|c|}
\hline Sub-Hypotheses & $\begin{array}{l}\text { Board } \\
\text { Chair }\end{array}$ & $\begin{array}{c}\text { Presi- } \\
\text { dent }\end{array}$ & $\begin{array}{l}\text { Aca- } \\
\text { demic } \\
\text { VP }\end{array}$ & $\begin{array}{c}\text { Student } \\
\text { Service } \\
\text { VP }\end{array}$ & $\begin{array}{c}\text { Finance } \\
\text { VP }\end{array}$ & Total & $F$ & $P$ \\
\hline $\begin{array}{l}\text { 23. Comply with legal } \\
\text { mandates and laws in } \\
\text { addressing grievances } \\
M \\
S D \\
N\end{array}$ & $\begin{array}{r}4.33 \\
.77 \\
18\end{array}$ & $\begin{array}{r}4.59 \\
1.00 \\
17\end{array}$ & $\begin{array}{r}4.31 \\
.79 \\
16\end{array}$ & $\begin{array}{r}4.15 \\
.99 \\
13\end{array}$ & $\begin{array}{r}3.79 \\
1.05 \\
14\end{array}$ & $\begin{array}{r}4.26 \\
.93 \\
78\end{array}$ & 1.560 & .194 \\
\hline $\begin{array}{l}\text { 24. Help formulate and } \\
\text { adhere to institution's } \\
\text { educational } \\
\text { philosophy } \\
M \\
S D \\
N\end{array}$ & $\begin{array}{r}4.61 \\
.61 \\
18\end{array}$ & $\begin{array}{r}4.65 \\
.86 \\
17\end{array}$ & $\begin{array}{r}3.75 \\
1.39 \\
16\end{array}$ & $\begin{array}{r}4.69 \\
.48 \\
13\end{array}$ & $\begin{array}{r}4.50 \\
.65 \\
14\end{array}$ & $\begin{array}{r}4.44 \\
.92 \\
78\end{array}$ & 3.210 & .017 \\
\hline $\begin{array}{l}\text { 25. Delegate } \\
\text { responsibility, } \\
\text { authority, and needed } \\
\text { resources to } \\
\text { appropriate } \\
\text { administrators and } \\
\text { support their roles } \\
M \\
S D \\
N\end{array}$ & $\begin{array}{r}3.89 \\
1.02 \\
18\end{array}$ & $\begin{array}{r}4.71 \\
.77 \\
17\end{array}$ & $\begin{array}{r}4.13 \\
1.15 \\
16\end{array}$ & $\begin{array}{r}3.85 \\
1.46 \\
13\end{array}$ & $\begin{array}{r}4.07 \\
1.21 \\
14\end{array}$ & $\begin{array}{r}4.14 \\
1.14 \\
78\end{array}$ & 1.547 & .198 \\
\hline
\end{tabular}


Table 36. Composite ANOVA table for sub-hypotheses 26-30, showing means and standard deviations of subgroups for finance-related functions--actual roles

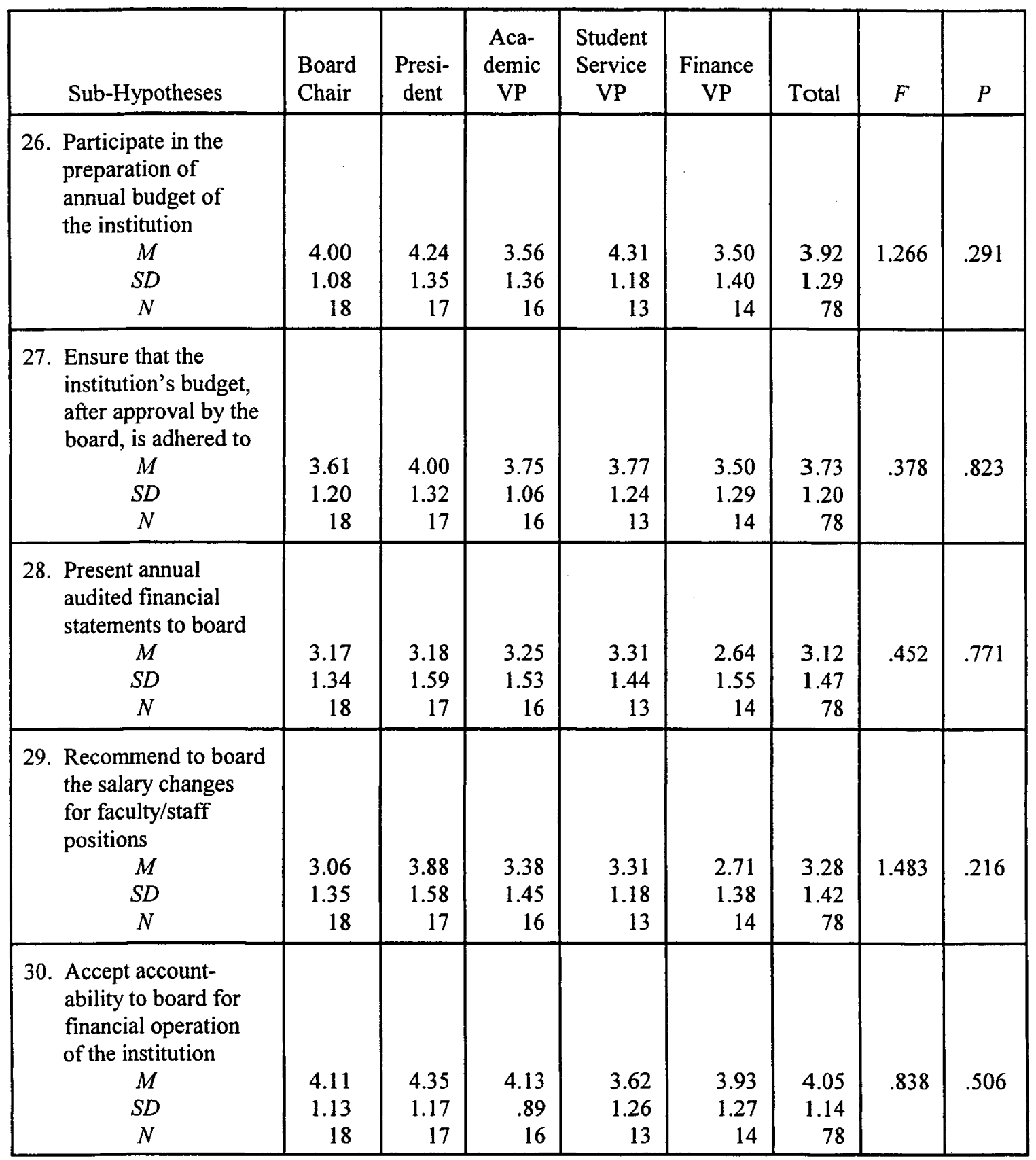


Table 37. Composite ANOVA table for sub-hypotheses 31-36, showing means and standard deviations of subgroups for physical facilities-related functions--actual roles

\begin{tabular}{|c|c|c|c|c|c|c|c|c|}
\hline Sub-Hypotheses & $\begin{array}{l}\text { Board } \\
\text { Chair }\end{array}$ & $\begin{array}{c}\text { Presi- } \\
\text { dent }\end{array}$ & $\begin{array}{l}\text { Aca- } \\
\text { demic } \\
\text { VP }\end{array}$ & $\begin{array}{c}\text { Student } \\
\text { Service } \\
\text { VP }\end{array}$ & $\begin{array}{l}\text { Finance } \\
\text { VP }\end{array}$ & Total & $F$ & $P$ \\
\hline $\begin{array}{l}\text { 31. Present to the board } \\
\text { the physical needs of } \\
\text { the institutions } \\
M \\
S D \\
N\end{array}$ & $\begin{array}{r}4.44 \\
.70 \\
18\end{array}$ & $\begin{array}{r}4.29 \\
1.10 \\
17\end{array}$ & $\begin{array}{r}4.00 \\
1.32 \\
16\end{array}$ & $\begin{array}{r}4.23 \\
1.17 \\
13\end{array}$ & $\begin{array}{r}3.21 \\
1.63 \\
14\end{array}$ & $\begin{array}{r}4.06 \\
1.24 \\
78\end{array}$ & 2.449 & .054 \\
\hline $\begin{array}{l}\text { 32. Direct the preparation } \\
\text { of plans for new } \\
\text { buildings and instal- } \\
\text { lations as needed } \\
M \\
S D \\
N\end{array}$ & $\begin{array}{r}4.06 \\
1.00 \\
18\end{array}$ & $\begin{array}{r}3.35 \\
1.54 \\
17\end{array}$ & $\begin{array}{r}3.69 \\
1.35 \\
16\end{array}$ & $\begin{array}{r}3.46 \\
1.66 \\
13\end{array}$ & $\begin{array}{r}2.79 \\
1.53 \\
14\end{array}$ & $\begin{array}{r}3.50 \\
1.44 \\
78\end{array}$ & 1.708 & .158 \\
\hline $\begin{array}{l}\text { 33. Plan for major repairs } \\
\text { and alterations of } \\
\text { plant, buildings and } \\
\text { equipment } \\
M \\
S D \\
N\end{array}$ & $\begin{array}{r}3.44 \\
1.25 \\
18 \\
\end{array}$ & $\begin{array}{r}2.65 \\
1.46 \\
17 \\
\end{array}$ & $\begin{array}{r}3.25 \\
1.29 \\
16 \\
\end{array}$ & $\begin{array}{r}3.23 \\
1.64 \\
13 \\
\end{array}$ & $\begin{array}{r}2.64 \\
1.28 \\
14 \\
\end{array}$ & $\begin{array}{r}3.05 \\
1.39 \\
78 \\
\end{array}$ & 1.176 & .329 \\
\hline $\begin{array}{l}\text { 34. Recommend to board } \\
\text { for equipment } \\
\text { acquisitions } \\
M \\
S D \\
N\end{array}$ & $\begin{array}{r}3.50 \\
1.42 \\
18\end{array}$ & $\begin{array}{r}3.00 \\
1.58 \\
17\end{array}$ & $\begin{array}{r}3.00 \\
1.59 \\
16\end{array}$ & $\begin{array}{r}3.15 \\
1.52 \\
13\end{array}$ & $\begin{array}{r}2.50 \\
1.40 \\
14\end{array}$ & $\begin{array}{r}3.05 \\
1.50 \\
78\end{array}$ & .892 & .473 \\
\hline $\begin{array}{l}\text { 35. Ensure that the } \\
\text { physical plant master } \\
\text { plans are current and } \\
\text { are followed as } \\
\text { closely as possible } \\
M \\
S D \\
N\end{array}$ & $\begin{array}{r}4.06 \\
1.63 \\
18\end{array}$ & $\begin{array}{r}3.94 \\
1.30 \\
17\end{array}$ & $\begin{array}{r}3.63 \\
1.26 \\
16\end{array}$ & $\begin{array}{r}3.23 \\
1.30 \\
13\end{array}$ & $\begin{array}{r}3.07 \\
1.07 \\
14\end{array}$ & $\begin{array}{r}3.63 \\
1.36 \\
78\end{array}$ & 1.582 & .188 \\
\hline $\begin{array}{l}\text { 36. Ascertain that the } \\
\text { campus grounds are } \\
\text { well-kept through } \\
\text { appropriate officer } \\
M \\
S D \\
N\end{array}$ & $\begin{array}{c}3.78 \\
1.22 \\
18\end{array}$ & $\begin{array}{r}4.18 \\
1.13 \\
17\end{array}$ & $\begin{array}{r}3.56 \\
1.31 \\
16\end{array}$ & $\begin{array}{r}3.69 \\
1.11 \\
13\end{array}$ & $\begin{array}{r}3.36 \\
1.08 \\
14\end{array}$ & $\begin{array}{r}3.73 \\
1.18 \\
78\end{array}$ & 1.051 & .387 \\
\hline
\end{tabular}


Table 38. Composite ANOVA table for sub-hypotheses $37-42$, showing means and standard deviations of subgroups for community- and alumni-related functions--actual roles

\begin{tabular}{|c|c|c|c|c|c|c|c|c|}
\hline Sub-Hypotheses & $\begin{array}{l}\text { Board } \\
\text { Chair }\end{array}$ & $\begin{array}{c}\text { Presi- } \\
\text { dent }\end{array}$ & $\begin{array}{l}\text { Aca- } \\
\text { demic } \\
\text { VP }\end{array}$ & $\begin{array}{l}\text { Student } \\
\text { Service } \\
\text { VP }\end{array}$ & $\begin{array}{l}\text { Finance } \\
\text { VP }\end{array}$ & Total & $F$ & $P$ \\
\hline \multicolumn{9}{|l|}{$\begin{array}{l}\text { 37. Assist in raising } \\
\text { funds for support of } \\
\text { the institution }\end{array}$} \\
\hline$M$ & 4.06 & 4.24 & 3.94 & 4.31 & 3.93 & 4.09 & .314 & .868 \\
\hline$S D$ & 1.21 & 1.25 & 1.29 & 1.11 & .92 & 1.15 & & \\
\hline$N$ & 18 & 17 & 16 & 13 & 14 & 78 & & \\
\hline \multicolumn{9}{|l|}{$\begin{array}{l}\text { 38. Represent institution } \\
\text { to the churches of } \\
\text { the constituency }\end{array}$} \\
\hline$M$ & 4.28 & 4.88 & 3.94 & 4.69 & 4.21 & 4.40 & 3.042 & .022 \\
\hline$S D$ & .75 & .33 & 1.34 & .85 & .80 & .92 & & \\
\hline$N$ & 18 & 17 & 16 & 13 & 14 & 78 & & \\
\hline \multicolumn{9}{|l|}{$\begin{array}{l}\text { 39. Present reports about } \\
\text { institution to } \\
\text { community and } \\
\text { alumni }\end{array}$} \\
\hline$M$ & 4.61 & 4.76 & 4.06 & 4.31 & 3.50 & 4.28 & 3.837 & .007 \\
\hline$S D$ & .50 & .66 & 1.18 & 1.32 & 1.22 & 1.07 & & \\
\hline$N$ & 18 & 17 & 16 & 13 & 14 & 78 & & \\
\hline \multicolumn{9}{|l|}{$\begin{array}{l}\text { 40. Represent institution } \\
\text { as its official spokes- }\end{array}$} \\
\hline$M$ & 4.39 & 4.76 & 4.44 & 4.77 & 4.07 & 4.49 & 1.666 & .167 \\
\hline$S D$ & 1.20 & .75 & .73 & .60 & .83 & .88 & & \\
\hline$N$ & 18 & 17 & 16 & 13 & 14 & 78 & & \\
\hline \multicolumn{9}{|l|}{$\begin{array}{l}\text { 41. Associate closely } \\
\text { with alumni groups }\end{array}$} \\
\hline$M$ & 3.83 & 4.35 & 4.19 & 4.15 & 3.71 & 4.05 & 1.101 & .363 \\
\hline$S D$ & 1.04 & .86 & .83 & 1.28 & .99 & 1.01 & & \\
\hline$N$ & 18 & 17 & 16 & 13 & 14 & 78 & & \\
\hline \multicolumn{9}{|l|}{$\begin{array}{l}\text { 42. Listen to constituency } \\
\text { and alumni }\end{array}$} \\
\hline$M$ & 4.06 & 4.76 & 3.94 & 4.31 & 4.07 & 4.23 & 1.716 & .156 \\
\hline$S D$ & 1.26 & .44 & .93 & 1.38 & .92 & 1.04 & & \\
\hline$N$ & 18 & 17 & 16 & 13 & 14 & 78 & & \\
\hline
\end{tabular}


Table 39. Composite ANOVA table for sub-hypotheses $43-47$, showing means and standard deviations of subgroups for spiritual life-related functions--actual roles

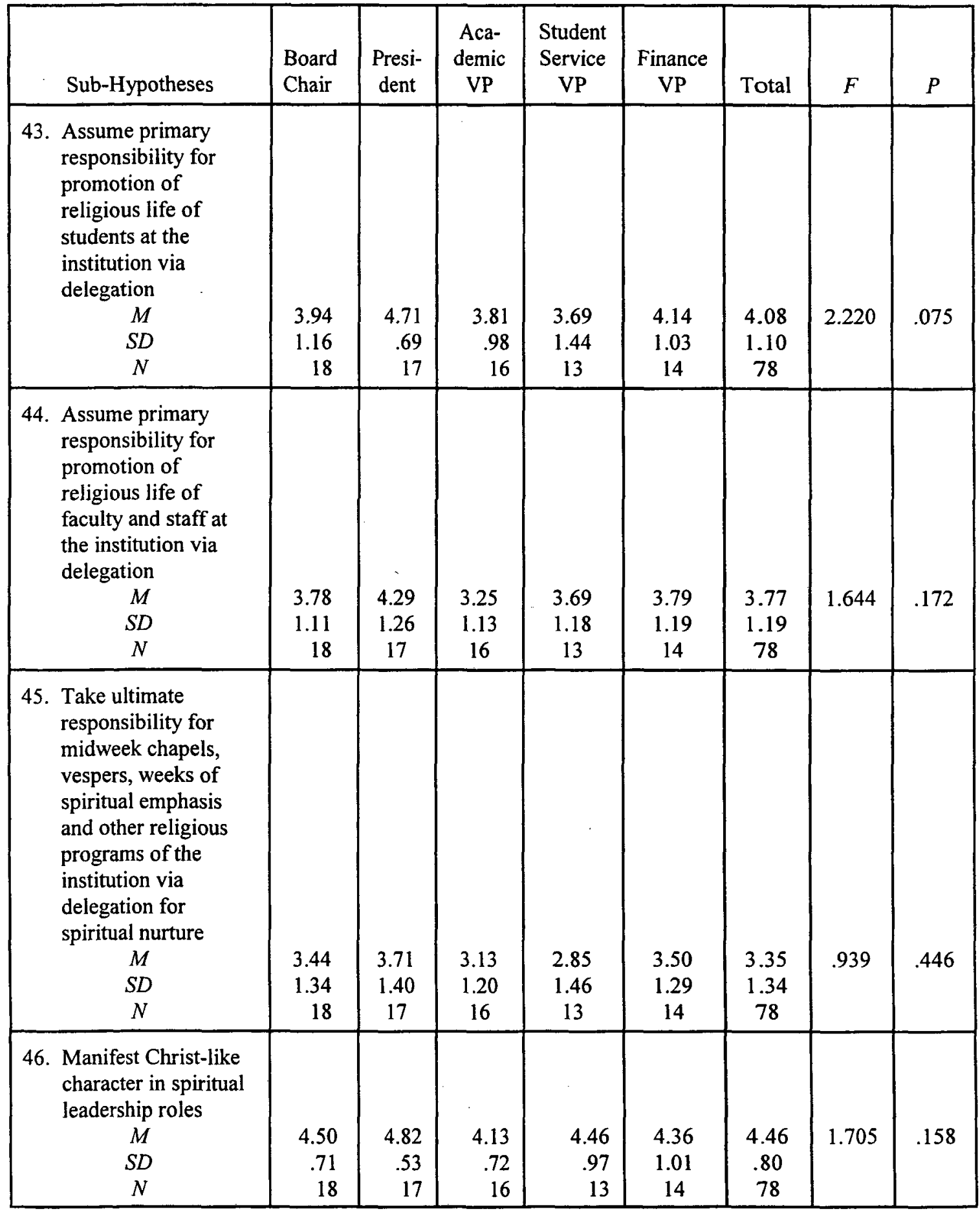


Table 39--Continued.

\begin{tabular}{|l|r|r|r|r|r|r|r|r|}
\hline Sub-Hypotheses & $\begin{array}{c}\text { Board } \\
\text { Chair }\end{array}$ & $\begin{array}{c}\text { Presi- } \\
\text { dent }\end{array}$ & $\begin{array}{c}\text { Aca- } \\
\text { demic } \\
\text { VP }\end{array}$ & $\begin{array}{c}\text { Student } \\
\text { Service } \\
\text { VP }\end{array}$ & $\begin{array}{c}\text { Finance } \\
\text { VP }\end{array}$ & Total & $F$ & $P$ \\
\hline 47. Focus on the & & & & & & & & \\
Christian philosophy & & & & & & & & \\
of the institution & & & & & & & & \\
$M$ & 4.72 & 4.94 & 4.56 & 4.54 & 4.36 & 4.64 & 1.281 & .285 \\
$S D$ & .57 & .24 & .73 & 1.20 & .93 & .77 & & \\
$N$ & 18 & 17 & 16 & 13 & 14 & 78 & & \\
\hline
\end{tabular}


APPENDIX H

TABLES $40-46$

COMPOSITE ANOVA RESULTS FOR EXPECTED ROLES

SHOWING MEANS AND STANDARD DEVIATIONS

FOR SUB-HYPOTHESES 1-47 
Table 40. Composite ANOVA table for sub-hypotheses 1-8, showing means and standard deviations of subgroups for student-related functions--expected roles

\begin{tabular}{|c|c|c|c|c|c|c|c|c|}
\hline Sub-Hypotheses & $\begin{array}{l}\text { Board } \\
\text { Chair }\end{array}$ & $\begin{array}{l}\text { Presi- } \\
\text { dent }\end{array}$ & $\begin{array}{l}\text { Aca- } \\
\text { demic } \\
\text { VP }\end{array}$ & $\begin{array}{l}\text { Student } \\
\text { Service } \\
\text { VP }\end{array}$ & $\begin{array}{l}\text { Finance } \\
\text { VP }\end{array}$ & Total & $F$ & $P$ \\
\hline \multicolumn{9}{|l|}{$\begin{array}{l}\text { 1. Participate in student } \\
\text { recruitment }\end{array}$} \\
\hline$M$ & 4.11 & 3.53 & 3.06 & 3.31 & 3.86 & 3.59 & 2.443 & .054 \\
\hline$S D$ & .90 & 1.18 & 1.12 & 1.32 & .86 & 1.12 & & \\
\hline$N$ & 18 & 17 & 16 & 13 & 14 & 78 & & \\
\hline \multicolumn{9}{|l|}{$\begin{array}{l}\text { 2. Work closely with } \\
\text { student government } \\
\text { leaders }\end{array}$} \\
\hline$M$ & 4.06 & 3.53 & 3.69 & 3.54 & 3.36 & 3.65 & .881 & .479 \\
\hline$S D$ & .80 & 1.28 & 1.20 & 1.27 & 1.15 & 1.14 & & \\
\hline$N$ & 18 & 17 & 16 & 13 & 14 & 78 & & \\
\hline \multicolumn{9}{|l|}{$\begin{array}{l}\text { 3. Dialogue frequently } \\
\text { with students }\end{array}$} \\
\hline$M$ & 4.56 & 4.00 & 3.94 & 4.23 & 4.21 & 4.19 & 1.374 & .251 \\
\hline$S D$ & .51 & 1.12 & .85 & 1.01 & .70 & .87 & & \\
\hline$N$ & 18 & 17 & 16 & 13 & 14 & 78 & & \\
\hline $\begin{array}{l}\text { 4. Visit students } \\
\text { periodically in the } \\
\text { dorms during their } \\
\text { dorm worships }\end{array}$ & & & & & & & & \\
\hline$M$ & 3.44 & 3.12 & 2.69 & 2.62 & 3.29 & 3.05 & 1.343 & .262 \\
\hline$S D$ & 1.29 & 1.27 & 1.14 & 1.26 & 1.20 & 1.25 & & \\
\hline$N$ & 18 & 17 & 16 & 13 & 14 & 78 & & \\
\hline \multicolumn{9}{|l|}{$\begin{array}{l}\text { 5. Take ultimate } \\
\text { responsibility for }\end{array}$} \\
\hline$M$ & 4.11 & 2.94 & 2.94 & 3.00 & 2.86 & 3.21 & 2.161 & .082 \\
\hline$S D$ & 1.28 & 1.39 & 1.57 & 1.73 & 1.56 & 1.54 & & \\
\hline$N$ & 18 & 17 & 16 & 13 & 14 & 78 & & \\
\hline \multicolumn{9}{|l|}{$\begin{array}{l}\text { 6. Teach some classes } \\
\text { to maintain contact } \\
\text { with students }\end{array}$} \\
\hline$M$ & 3.00 & 2.88 & 2.31 & 2.46 & 2.93 & 2.73 & .637 & .637 \\
\hline$S D$ & 1.68 & 1.58 & 1.20 & 1.61 & 1.54 & 1.52 & & \\
\hline$N$ & 18 & 17 & 16 & 13 & 14 & 78 & & \\
\hline
\end{tabular}


Table 40-Continued.

\begin{tabular}{|c|c|c|c|c|c|c|c|c|}
\hline Sub-Hypotheses & $\begin{array}{c}\text { Board } \\
\text { Chair }\end{array}$ & $\begin{array}{c}\text { Presi- } \\
\text { dent }\end{array}$ & $\begin{array}{c}\text { Aca- } \\
\text { demic } \\
\text { VP }\end{array}$ & $\begin{array}{c}\text { Student } \\
\text { Service } \\
\text { VP }\end{array}$ & $\begin{array}{c}\text { Finance } \\
\text { VP }\end{array}$ & Total & $F$ & $P$ \\
\hline $\begin{array}{c}\text { 7. Know every student } \\
\text { personally }\end{array}$ & & & & & & & & \\
$M$ & 2.83 & 2.24 & 2.13 & 2.62 & 2.21 & 2.41 & .869 & .487 \\
$S D$ & 1.54 & 1.20 & 1.26 & 1.45 & 1.12 & 1.32 & & \\
$N$ & 18 & 17 & 16 & 13 & 14 & 78 & & \\
\hline 8. Serve as mentor & & & & & & & & \\
of students & & & & & & & & \\
$M$ & 3.67 & 3.24 & 2.56 & 4.00 & 3.29 & 3.33 & 2.958 & .025 \\
$S D$ & 1.24 & 1.30 & 1.26 & 1.00 & 1.20 & 1.28 & & \\
$N$ & 18 & 17 & 16 & 13 & 14 & 78 & & \\
\hline
\end{tabular}


Table 41. Composite ANOVA table for sub-hypotheses 9-16, showing means and standard deviations of subgroups for employee-related functions--expected roles

\begin{tabular}{|c|c|c|c|c|c|c|c|c|}
\hline Sub-Hypotheses & $\begin{array}{l}\text { Board } \\
\text { Chair }\end{array}$ & $\begin{array}{l}\text { Presi- } \\
\text { dent }\end{array}$ & $\begin{array}{l}\text { Aca- } \\
\text { demic } \\
\text { VP }\end{array}$ & $\begin{array}{c}\text { Student } \\
\text { Service } \\
\text { VP }\end{array}$ & $\begin{array}{l}\text { Finance } \\
\text { VP }\end{array}$ & Total & $F$ & $P$ \\
\hline $\begin{array}{l}\text { 9. Participate in faculty } \\
\text { recruitment } \\
M \\
S D \\
N\end{array}$ & $\begin{array}{r}4.72 \\
.46 \\
18\end{array}$ & $\begin{array}{r}4.18 \\
.81 \\
17\end{array}$ & $\begin{array}{r}4.00 \\
1.10 \\
16\end{array}$ & $\begin{array}{r}4.08 \\
1.19 \\
13\end{array}$ & $\begin{array}{r}4.00 \\
1.04 \\
14\end{array}$ & $\begin{array}{r}4.22 \\
.95 \\
78\end{array}$ & 1.822 & .134 \\
\hline $\begin{array}{l}\text { 10. Serve as academic } \\
\text { leader of the faculty } \\
M \\
S D \\
N\end{array}$ & $\begin{array}{r}3.67 \\
1.41 \\
18\end{array}$ & $\begin{array}{r}3.24 \\
1.20 \\
17\end{array}$ & $\begin{array}{r}3.00 \\
1.21 \\
16\end{array}$ & $\begin{array}{r}3.3 \\
1.38 \\
13\end{array}$ & $\begin{array}{r}3.29 \\
1.14 \\
14\end{array}$ & $\begin{array}{r}3.31 \\
1.26 \\
78\end{array}$ & .604 & .661 \\
\hline $\begin{array}{l}\text { 11. Guard faculty's } \\
\text { academic freedom } \\
M \\
S D \\
N\end{array}$ & $\begin{array}{r}4.33 \\
.77 \\
18\end{array}$ & $\begin{array}{r}4.24 \\
.97 \\
17\end{array}$ & $\begin{array}{r}4.06 \\
.85 \\
16\end{array}$ & $\begin{array}{r}3.85 \\
.90 \\
13\end{array}$ & $\begin{array}{r}4.00 \\
.88 \\
14\end{array}$ & $\begin{array}{r}4.12 \\
.87 \\
78\end{array}$ & .745 & .565 \\
\hline $\begin{array}{l}\text { 12. Have responsibility } \\
\text { in submitting } \\
\text { proposed changes in } \\
\text { academic matters to } \\
\text { faculty for approval } \\
M \\
S D \\
N\end{array}$ & $\begin{array}{r}3.78 \\
1.22 \\
18\end{array}$ & $\begin{array}{r}2.65 \\
1.37 \\
17\end{array}$ & $\begin{array}{r}2.56 \\
1.26 \\
16\end{array}$ & $\begin{array}{r}3.62 \\
1.33 \\
13\end{array}$ & $\begin{array}{r}3.50 \\
1.40 \\
14\end{array}$ & $\begin{array}{r}3.21 \\
1.38 \\
78\end{array}$ & 3.084 & .021 \\
\hline $\begin{array}{l}\text { 13. Work with faculty in } \\
\text { reviewing accrediting } \\
\text { agency's reports } \\
M \\
S D \\
N\end{array}$ & $\begin{array}{r}4.50 \\
.71 \\
18\end{array}$ & $\begin{array}{r}4.12 \\
1.05 \\
17\end{array}$ & $\begin{array}{r}3.94 \\
1.24 \\
16\end{array}$ & $\begin{array}{r}4.23 \\
.83 \\
13\end{array}$ & $\begin{array}{r}4.36 \\
1.15 \\
14\end{array}$ & $\begin{array}{r}4.23 \\
1.00 \\
78\end{array}$ & .762 & .553 \\
\hline $\begin{array}{l}\text { 14. Recommend to board } \\
\text { for hiring, promotion, } \\
\text { demotion and } \\
\text { dismissal of faculty } \\
\text { and staff } \\
M \\
S D \\
N\end{array}$ & $\begin{array}{r}4.17 \\
1.15 \\
18\end{array}$ & $\begin{array}{r}4.35 \\
1.22 \\
17\end{array}$ & $\begin{array}{r}4.69 \\
.79 \\
16\end{array}$ & $\begin{array}{r}4.62 \\
.87 \\
13\end{array}$ & $\begin{array}{r}4.29 \\
.73 \\
14\end{array}$ & $\begin{array}{r}4.41 \\
.99 \\
78\end{array}$ & .793 & .534 \\
\hline
\end{tabular}


Table 41-Continued.

\begin{tabular}{|c|r|r|r|r|r|r|r|r|}
\hline Sub-Hypotheses & $\begin{array}{c}\text { Board } \\
\text { Chair }\end{array}$ & $\begin{array}{c}\text { Presi- } \\
\text { dent }\end{array}$ & $\begin{array}{c}\text { Aca- } \\
\text { demic } \\
\text { VP }\end{array}$ & $\begin{array}{c}\text { Student } \\
\text { Service } \\
\text { VP }\end{array}$ & $\begin{array}{c}\text { Finance } \\
\text { VP }\end{array}$ & Total & $F$ & $P$ \\
\hline $\begin{array}{c}\text { 15. Collaborate for } \\
\text { advanced in-service } \\
\text { training for faculty }\end{array}$ & & & & & & & & \\
and staff & & & & & & & & \\
$M$ & 4.11 & 3.65 & 3.00 & 4.08 & 3.43 & 3.65 & 2.441 & .054 \\
$S D$ & .96 & 1.11 & 1.46 & .95 & 1.34 & 1.23 & & \\
$N$ & 18 & 17 & 16 & 13 & 14 & 78 & & \\
\hline 16. Carry out policies & & & & & & & & \\
and regulations of & & & & & & & & \\
faculty and staff & & & & & & & & \\
$M$ & 4.11 & 4.06 & 3.75 & 3.92 & 3.57 & 3.90 & .473 & .756 \\
$S D$ & 1.08 & 1.34 & 1.53 & 1.19 & 1.22 & 1.26 & & \\
$N$ & 18 & 17 & 16 & 13 & 14 & 78 & & \\
\hline
\end{tabular}


Table 42. Composite ANOVA table for sub-hypotheses 17-25, showing means and standard deviations of subgroups for general administrative functions--expected roles

\begin{tabular}{|c|c|c|c|c|c|c|c|c|}
\hline Sub-Hypotheses & $\begin{array}{l}\text { Board } \\
\text { Chair }\end{array}$ & $\begin{array}{l}\text { Presi- } \\
\text { dent }\end{array}$ & $\begin{array}{l}\text { Aca- } \\
\text { demic } \\
\text { VP }\end{array}$ & $\begin{array}{c}\text { Student } \\
\text { Service } \\
\text { VP }\end{array}$ & $\begin{array}{c}\text { Finance } \\
\text { VP }\end{array}$ & Total & $F$ & $P$ \\
\hline $\begin{array}{l}\text { 17. Prepare agenda for } \\
\text { board of trustees' } \\
\text { meetings } \\
M \\
S D \\
N\end{array}$ & $\begin{array}{r}4.72 \\
.96 \\
18\end{array}$ & $\begin{array}{r}5.00 \\
.00 \\
17\end{array}$ & $\begin{array}{r}4.94 \\
.25 \\
16\end{array}$ & $\begin{array}{r}4.77 \\
.60 \\
13\end{array}$ & $\begin{array}{r}4.57 \\
.65 \\
14\end{array}$ & $\begin{array}{r}4.81 \\
.60 \\
78\end{array}$ & 1.271 & .289 \\
\hline $\begin{array}{l}\text { 18. Preside over general } \\
\text { faculty meetings } \\
M \\
S D \\
N\end{array}$ & $\begin{array}{r}4.61 \\
1.04 \\
18\end{array}$ & $\begin{array}{r}4.06 \\
1.20 \\
17\end{array}$ & $\begin{array}{r}4.00 \\
1.46 \\
16\end{array}$ & $\begin{array}{r}3.62 \\
1.50 \\
13\end{array}$ & $\begin{array}{r}3.64 \\
1.50 \\
14\end{array}$ & $\begin{array}{r}4.03 \\
1.35 \\
78\end{array}$ & 1.471 & .220 \\
\hline $\begin{array}{l}\text { 19. Take responsibility in } \\
\text { structuring student } \\
\text { admission standards } \\
M \\
S D \\
N\end{array}$ & $\begin{array}{r}4.06 \\
.87 \\
18\end{array}$ & $\begin{array}{r}2.71 \\
1.31 \\
17\end{array}$ & $\begin{array}{r}3.06 \\
1.24 \\
16\end{array}$ & $\begin{array}{r}3.15 \\
1.41 \\
13\end{array}$ & $\begin{array}{r}3.29 \\
1.14 \\
14\end{array}$ & $\begin{array}{r}3.27 \\
1.26 \\
78\end{array}$ & 3.052 & .022 \\
\hline $\begin{array}{l}\text { 20. Take responsibility in } \\
\text { strategic planning } \\
\text { for the institution } \\
M \\
S D \\
N\end{array}$ & $\begin{array}{r}4.83 \\
.38 \\
18\end{array}$ & $\begin{array}{r}4.59 \\
1.00 \\
17\end{array}$ & $\begin{array}{r}4.88 \\
.34 \\
16\end{array}$ & $\begin{array}{r}4.69 \\
.63 \\
13\end{array}$ & $\begin{array}{r}4.79 \\
.43 \\
14\end{array}$ & $\begin{array}{r}4.76 \\
.61 \\
78\end{array}$ & .583 & .676 \\
\hline $\begin{array}{l}\text { 21. Take a leading role to } \\
\text { prepare, revise, and } \\
\text { follow the school's } \\
\text { organizational chart. } \\
M \\
S D \\
N\end{array}$ & $\begin{array}{r}4.78 \\
.43 \\
18\end{array}$ & $\begin{array}{r}4.41 \\
1.06 \\
17\end{array}$ & $\begin{array}{r}4.56 \\
.73 \\
16\end{array}$ & $\begin{array}{r}4.54 \\
.66 \\
13\end{array}$ & $\begin{array}{r}4.29 \\
.73 \\
14\end{array}$ & $\begin{array}{r}4.53 \\
.75 \\
78\end{array}$ & .971 & .429 \\
\hline $\begin{array}{l}\text { 22. Foster research and } \\
\text { intellectual climate } \\
\text { for the institution } \\
M \\
S D \\
N\end{array}$ & $\begin{array}{r}4.17 \\
.92 \\
18\end{array}$ & $\begin{array}{r}4.18 \\
.88 \\
17\end{array}$ & $\begin{array}{r}4.19 \\
.91 \\
16\end{array}$ & $\begin{array}{r}4.54 \\
.66 \\
13\end{array}$ & $\begin{array}{r}4.36 \\
.74 \\
14\end{array}$ & $\begin{array}{r}4.27 \\
.83 \\
78\end{array}$ & .525 & .717 \\
\hline
\end{tabular}


Table 42-Continued.

\begin{tabular}{|c|c|c|c|c|c|c|c|c|}
\hline Sub-Hypotheses & $\begin{array}{l}\text { Board } \\
\text { Chair }\end{array}$ & $\begin{array}{l}\text { Presi- } \\
\text { dent }\end{array}$ & $\begin{array}{l}\text { Aca- } \\
\text { demic } \\
\text { VP }\end{array}$ & $\begin{array}{c}\text { Student } \\
\text { Service } \\
\text { VP }\end{array}$ & $\begin{array}{c}\text { Finance } \\
\text { VP }\end{array}$ & Total & $F$ & $P$ \\
\hline $\begin{array}{l}\text { 23. Comply with legal } \\
\text { mandates and laws in } \\
\text { addressing grievances } \\
M \\
S D \\
N\end{array}$ & $\begin{array}{r}4.67 \\
.49 \\
18\end{array}$ & $\begin{array}{r}4.65 \\
1.00 \\
17\end{array}$ & $\begin{array}{r}4.69 \\
.60 \\
16\end{array}$ & $\begin{array}{r}4.77 \\
.44 \\
13\end{array}$ & $\begin{array}{r}4.21 \\
.97 \\
14\end{array}$ & $\begin{array}{r}4.60 \\
.74 \\
78\end{array}$ & 1.230 & .306 \\
\hline $\begin{array}{l}\text { 24. Help formulate and } \\
\text { adhere to institution's } \\
\text { educational } \\
\text { philosophy } \\
M \\
S D \\
N\end{array}$ & $\begin{array}{r}5.00 \\
.00 \\
18\end{array}$ & $\begin{array}{r}4.76 \\
.56 \\
17\end{array}$ & $\begin{array}{r}4.88 \\
.34 \\
16\end{array}$ & $\begin{array}{r}4.92 \\
.28 \\
13\end{array}$ & $\begin{array}{r}4.79 \\
.43 \\
14\end{array}$ & $\begin{array}{r}4.87 \\
.37 \\
78\end{array}$ & 1.138 & .346 \\
\hline $\begin{array}{l}\text { 25. Delegate } \\
\text { responsibility, } \\
\text { authority, and needed } \\
\text { resources to } \\
\text { appropriate } \\
\text { administrators and } \\
\text { support their roles } \\
M \\
S D \\
N\end{array}$ & $\begin{array}{r}4.44 \\
.70 \\
18\end{array}$ & $\begin{array}{r}4.76 \\
.44 \\
17\end{array}$ & $\begin{array}{r}4.94 \\
.25 \\
16\end{array}$ & $\begin{array}{r}4.85 \\
.38 \\
13\end{array}$ & $\begin{array}{r}4.71 \\
.61 \\
14\end{array}$ & $\begin{array}{r}4.73 \\
.53 \\
78\end{array}$ & 2.264 & .070 \\
\hline
\end{tabular}


Table 43. Composite ANOVA table for sub-hypotheses 26-30, showing means and standard deviations of subgroups for finance-related functions-expected roles

\begin{tabular}{|c|c|c|c|c|c|c|c|c|}
\hline Sub-Hypotheses & $\begin{array}{l}\text { Board } \\
\text { Chair }\end{array}$ & $\begin{array}{l}\text { Presi- } \\
\text { dent }\end{array}$ & $\begin{array}{l}\text { Aca- } \\
\text { demic } \\
\text { VP }\end{array}$ & $\begin{array}{c}\text { Student } \\
\text { Service } \\
\text { VP }\end{array}$ & $\begin{array}{c}\text { Finance } \\
\text { VP }\end{array}$ & Total & $F$ & $P$ \\
\hline $\begin{array}{l}\text { 26. Participate in the } \\
\text { preparation of } \\
\text { annual budget of the } \\
\text { institution } \\
M \\
S D \\
N\end{array}$ & $\begin{array}{r}4.72 \\
.46 \\
18\end{array}$ & $\begin{array}{r}4.47 \\
1.23 \\
17\end{array}$ & $\begin{array}{r}4.50 \\
.63 \\
16\end{array}$ & $\begin{array}{r}4.54 \\
.66 \\
13\end{array}$ & $\begin{array}{r}3.64 \\
1.08 \\
14\end{array}$ & $\begin{array}{r}4.40 \\
.92 \\
78\end{array}$ & 3.492 & .012 \\
\hline $\begin{array}{l}\text { 27. Ensure that the } \\
\text { institution's budget, } \\
\text { after approval by the } \\
\text { board, is adhered to } \\
M \\
S D \\
N\end{array}$ & $\begin{array}{c}4.67 \\
.49 \\
18\end{array}$ & $\begin{array}{r}4.24 \\
1.30 \\
17\end{array}$ & $\begin{array}{r}4.69 \\
.60 \\
16\end{array}$ & $\begin{array}{r}4.38 \\
.96 \\
13\end{array}$ & $\begin{array}{r}4.21 \\
.80 \\
14\end{array}$ & $\begin{array}{r}4.45 \\
.88 \\
78\end{array}$ & 1.098 & .364 \\
\hline $\begin{array}{l}\text { 28. Present institution's } \\
\text { annual audited } \\
\text { financial statements } \\
\text { to board } \\
M \\
S D \\
N\end{array}$ & $\begin{array}{r}3.61 \\
1.50 \\
18\end{array}$ & $\begin{array}{r}3.18 \\
1.59 \\
17\end{array}$ & $\begin{array}{r}3.81 \\
1.33 \\
16\end{array}$ & $\begin{array}{r}3.46 \\
1.45 \\
13\end{array}$ & $\begin{array}{r}2.79 \\
1.63 \\
14\end{array}$ & $\begin{array}{r}3.38 \\
1.50 \\
78\end{array}$ & 1.073 & .376 \\
\hline $\begin{array}{l}\text { 29. Recommend to board } \\
\text { the salary changes for } \\
\text { faculty/staff positions } \\
M \\
S D \\
N\end{array}$ & $\begin{array}{r}3.22 \\
1.40 \\
18\end{array}$ & $\begin{array}{r}3.94 \\
1.52 \\
17\end{array}$ & $\begin{array}{r}4.06 \\
1.18 \\
16\end{array}$ & $\begin{array}{r}4.00 \\
1.22 \\
13\end{array}$ & $\begin{array}{r}3.29 \\
1.54 \\
14\end{array}$ & $\begin{array}{r}3.69 \\
1.40 \\
78\end{array}$ & 1.405 & .241 \\
\hline $\begin{array}{l}\text { 30. Accept accountability } \\
\text { to board for financial } \\
\text { operation of the } \\
\text { institution } \\
M \\
S D \\
N \\
\end{array}$ & $\begin{array}{r}4.78 \\
.43 \\
18 \\
\end{array}$ & $\begin{array}{r}4.47 \\
1.12 \\
17 \\
\end{array}$ & $\begin{array}{r}4.81 \\
.54 \\
16 \\
\end{array}$ & $\begin{array}{r}4.38 \\
1.12 \\
13 \\
\end{array}$ & $\begin{array}{r}4.57 \\
.85 \\
14 \\
\end{array}$ & $\begin{array}{r}4.62 \\
.84 \\
78 \\
\end{array}$ & .758 & .556 \\
\hline
\end{tabular}


Table 44. Composite ANOVA table for sub-hypotheses 31-36, showing means and standard deviations of subgroups for physical facilities-related functions--expected roles

\begin{tabular}{|c|c|c|c|c|c|c|c|c|}
\hline Sub-Hypotheses & $\begin{array}{l}\text { Board } \\
\text { Chair }\end{array}$ & $\begin{array}{l}\text { Presi- } \\
\text { dent }\end{array}$ & $\begin{array}{l}\text { Aca- } \\
\text { demic } \\
\text { VP }\end{array}$ & $\begin{array}{l}\text { Student } \\
\text { Service } \\
\text { VP }\end{array}$ & $\begin{array}{l}\text { Finance } \\
\text { VP }\end{array}$ & Total & $F$ & $P$ \\
\hline $\begin{array}{l}\text { 31. Present to the board } \\
\text { the physical needs of } \\
\text { the institution } \\
M \\
S D \\
N\end{array}$ & $\begin{array}{r}4.61 \\
.61 \\
18\end{array}$ & $\begin{array}{r}4.30 \\
1.10 \\
17\end{array}$ & $\begin{array}{r}4.69 \\
1.01 \\
16\end{array}$ & $\begin{array}{r}4.54 \\
1.13 \\
13\end{array}$ & $\begin{array}{r}3.43 \\
1.55 \\
14\end{array}$ & $\begin{array}{r}4.33 \\
1.16 \\
78\end{array}$ & 3.203 & .018 \\
\hline $\begin{array}{l}\text { 32. Direct the preparation } \\
\text { of plans for new } \\
\text { buildings and instal- } \\
\text { lations as needed } \\
M \\
S D \\
N\end{array}$ & $\begin{array}{r}4.11 \\
.90 \\
18\end{array}$ & $\begin{array}{r}3.41 \\
1.28 \\
17\end{array}$ & $\begin{array}{r}4.31 \\
.95 \\
16\end{array}$ & $\begin{array}{r}4.00 \\
1.15 \\
13\end{array}$ & $\begin{array}{r}3.07 \\
1.49 \\
14\end{array}$ & $\begin{array}{r}3.79 \\
1.22 \\
78\end{array}$ & 3.056 & .022 \\
\hline $\begin{array}{l}\text { 33. Plan for major repairs } \\
\text { and alterations of } \\
\text { plants, buildings and } \\
\text { equipment } \\
M \\
S D \\
N\end{array}$ & $\begin{array}{c}3.50 \\
1.20 \\
18\end{array}$ & $\begin{array}{r}2.65 \\
1.46 \\
17\end{array}$ & $\begin{array}{r}3.44 \\
1.26 \\
16\end{array}$ & $\begin{array}{r}3.54 \\
1.39 \\
13\end{array}$ & $\begin{array}{r}2.71 \\
1.27 \\
14\end{array}$ & $\begin{array}{r}3.17 \\
1.34 \\
78\end{array}$ & 1.794 & .139 \\
\hline $\begin{array}{l}\text { 34. Recommend to board } \\
\text { for equipment } \\
\text { acquisitions } \\
M \\
S D \\
N\end{array}$ & $\begin{array}{c}3.37 \\
1.64 \\
18\end{array}$ & $\begin{array}{r}3.12 \\
1.65 \\
17\end{array}$ & $\begin{array}{r}3.75 \\
1.53 \\
16\end{array}$ & $\begin{array}{r}3.38 \\
1.45 \\
13\end{array}$ & $\begin{array}{r}2.79 \\
1.37 \\
14\end{array}$ & $\begin{array}{r}3.36 \\
1.55 \\
78\end{array}$ & 1.021 & .402 \\
\hline $\begin{array}{l}\text { 35. Ensure that physical } \\
\text { plant master plans are } \\
\text { current and are } \\
\text { followed as closely as } \\
\text { possible } \\
M \\
S D \\
N\end{array}$ & $\begin{array}{c}4.28 \\
1.07 \\
18\end{array}$ & $\begin{array}{r}4.12 \\
1.22 \\
17\end{array}$ & $\begin{array}{r}4.19 \\
1.22 \\
16\end{array}$ & $\begin{array}{r}4.15 \\
.80 \\
13\end{array}$ & $\begin{array}{r}3.64 \\
1.00 \\
14\end{array}$ & $\begin{array}{r}4.09 \\
1.08 \\
78\end{array}$ & .769 & .549 \\
\hline $\begin{array}{l}\text { 36. Ascertain that the } \\
\text { campus grounds are } \\
\text { well-kept through } \\
\text { appropriate officer } \\
M \\
S D \\
N\end{array}$ & $\begin{array}{c}4.11 \\
1.13 \\
18\end{array}$ & $\begin{array}{r}4.12 \\
1.22 \\
17\end{array}$ & $\begin{array}{r}4.31 \\
.95 \\
16\end{array}$ & $\begin{array}{r}3.85 \\
1.14 \\
13\end{array}$ & $\begin{array}{r}3.71 \\
1.20 \\
14\end{array}$ & $\begin{array}{r}4.04 \\
1.12 \\
78\end{array}$ & .655 & .625 \\
\hline
\end{tabular}


Table 45. Composite ANOVA table for sub-hypotheses $37-42$, showing means and standard deviations of subgroups for communityand alumni-related functions--expected roles

\begin{tabular}{|c|c|c|c|c|c|c|c|c|}
\hline Sub-Hypotheses & $\begin{array}{l}\text { Board } \\
\text { Chair }\end{array}$ & $\begin{array}{l}\text { Presi- } \\
\text { dent }\end{array}$ & $\begin{array}{l}\text { Aca- } \\
\text { demic } \\
\text { VP }\end{array}$ & $\begin{array}{c}\text { Student } \\
\text { Service } \\
\text { VP }\end{array}$ & $\begin{array}{c}\text { Finance } \\
\text { VP }\end{array}$ & Total & $F$ & $P$ \\
\hline $\begin{array}{l}\text { 37. Assist in raising } \\
\text { funds for support of } \\
\text { the institution } \\
M \\
S D \\
N\end{array}$ & $\begin{array}{r}4.78 \\
.43 \\
18\end{array}$ & $\begin{array}{r}4.65 \\
1.00 \\
17\end{array}$ & $\begin{array}{r}4.94 \\
.25 \\
16\end{array}$ & $\begin{array}{r}4.77 \\
.83 \\
13\end{array}$ & $\begin{array}{r}4.71 \\
.47 \\
14\end{array}$ & $\begin{array}{r}4.77 \\
.64 \\
78\end{array}$ & .440 & .779 \\
\hline $\begin{array}{l}\text { 38. Represent institution } \\
\text { to the churches of } \\
\text { the constituency } \\
M \\
S D \\
N\end{array}$ & $\begin{array}{r}4.89 \\
.32 \\
18\end{array}$ & $\begin{array}{r}4.94 \\
.24 \\
17\end{array}$ & $\begin{array}{r}4.69 \\
.44 \\
16\end{array}$ & $\begin{array}{r}5.00 \\
.00 \\
13\end{array}$ & $\begin{array}{r}4.71 \\
.611 \\
14\end{array}$ & $\begin{array}{r}4.85 \\
.40 \\
78\end{array}$ & 1.889 & .121 \\
\hline $\begin{array}{l}\text { 39. Present reports about } \\
\text { institution to } \\
\text { community and } \\
\text { alumni } \\
M \\
S D \\
N\end{array}$ & $\begin{array}{r}4.78 \\
.43 \\
18\end{array}$ & $\begin{array}{r}4.82 \\
.53 \\
17\end{array}$ & $\begin{array}{r}4.63 \\
.62 \\
16\end{array}$ & $\begin{array}{r}4.85 \\
.38 \\
13\end{array}$ & $\begin{array}{r}4.21 \\
.97 \\
14\end{array}$ & $\begin{array}{r}4.67 \\
.64 \\
78\end{array}$ & 2.634 & .041 \\
\hline $\begin{array}{l}\text { 40. Represent institution } \\
\text { as its official } \\
\text { spokesperson to } \\
\text { public } \\
M \\
S D \\
N\end{array}$ & $\begin{array}{c}4.72 \\
.96 \\
18\end{array}$ & $\begin{array}{r}4.76 \\
.75 \\
17\end{array}$ & $\begin{array}{r}4.88 \\
.34 \\
16\end{array}$ & $\begin{array}{r}4.92 \\
.28 \\
13\end{array}$ & $\begin{array}{r}4.50 \\
.85 \\
14\end{array}$ & $\begin{array}{r}4.76 \\
.71 \\
78\end{array}$ & .757 & .556 \\
\hline $\begin{array}{l}\text { 41. Associate closely } \\
\text { with alumni groups } \\
M \\
S D \\
N\end{array}$ & $\begin{array}{r}4.56 \\
.62 \\
18\end{array}$ & $\begin{array}{r}4.53 \\
.62 \\
17\end{array}$ & $\begin{array}{r}4.56 \\
.63 \\
16\end{array}$ & $\begin{array}{r}4.46 \\
.66 \\
13\end{array}$ & $\begin{array}{r}4.43 \\
.65 \\
14\end{array}$ & $\begin{array}{r}4.51 \\
.62 \\
78\end{array}$ & .131 & .970 \\
\hline $\begin{array}{l}\text { 42. Listen to constituency } \\
\text { and alumni } \\
M \\
S D \\
N\end{array}$ & $\begin{array}{r}4.78 \\
.43 \\
18\end{array}$ & $\begin{array}{r}4.94 \\
.24 \\
17\end{array}$ & $\begin{array}{r}4.63 \\
.50 \\
16\end{array}$ & $\begin{array}{r}4.85 \\
.38 \\
13\end{array}$ & $\begin{array}{r}4.50 \\
.65 \\
14\end{array}$ & $\begin{array}{r}4.74 \\
.47 \\
78\end{array}$ & 2.285 & .068 \\
\hline
\end{tabular}


Table 46. Composite ANOVA table sub-hypotheses 43-47, showing means and standard deviations of subgroups for spiritual life-related functions--expected roles

\begin{tabular}{|c|c|c|c|c|c|c|c|c|}
\hline Sub-Hypotheses & $\begin{array}{l}\text { Board } \\
\text { Chair }\end{array}$ & $\begin{array}{l}\text { Presi- } \\
\text { dent }\end{array}$ & $\begin{array}{l}\text { Aca- } \\
\text { demic } \\
\text { VP }\end{array}$ & $\begin{array}{c}\text { Student } \\
\text { Service } \\
\text { VP }\end{array}$ & $\begin{array}{c}\text { Finance } \\
\text { VP }\end{array}$ & Total & $F$ & $P$ \\
\hline $\begin{array}{l}\text { 43. Assume primary } \\
\text { responsibility for } \\
\text { promotion of } \\
\text { religious life of } \\
\text { students at the } \\
\text { institution via } \\
\text { delegation } \\
M \\
S D \\
N\end{array}$ & $\begin{array}{r}4.83 \\
.51 \\
18\end{array}$ & $\begin{array}{r}4.71 \\
.59 \\
17\end{array}$ & $\begin{array}{r}4.38 \\
.72 \\
16\end{array}$ & $\begin{array}{r}3.85 \\
1.68 \\
13\end{array}$ & $\begin{array}{r}4.43 \\
.65 \\
14\end{array}$ & $\begin{array}{r}4.47 \\
.92 \\
78\end{array}$ & 2.742 & .035 \\
\hline $\begin{array}{l}\text { 44. Assume primary } \\
\text { responsibility for } \\
\text { promotion of } \\
\text { religious life of } \\
\text { faculty and staff at } \\
\text { the institution via } \\
\text { delegation } \\
M \\
S D \\
N\end{array}$ & $\begin{array}{r}4.72 \\
.57 \\
18\end{array}$ & $\begin{array}{r}4.41 \\
1.12 \\
17\end{array}$ & $\begin{array}{r}4.00 \\
1.03 \\
16\end{array}$ & $\begin{array}{r}4.31 \\
1.32 \\
13\end{array}$ & $\begin{array}{r}4.21 \\
.80 \\
14\end{array}$ & $\begin{array}{r}4.35 \\
.99 \\
78\end{array}$ & 1.236 & .303 \\
\hline $\begin{array}{l}\text { 45. Take ultimate } \\
\text { responsibility for } \\
\text { midweek chapels, } \\
\text { vespers, weeks of } \\
\text { spiritual emphasis } \\
\text { and other religious } \\
\text { programs of the } \\
\text { institution via } \\
\text { delegation for } \\
\text { spiritual nurture } \\
M \\
S D \\
N\end{array}$ & $\begin{array}{r}4.39 \\
1.09 \\
18\end{array}$ & $\begin{array}{r}3.82 \\
1.33 \\
17\end{array}$ & $\begin{array}{r}3.81 \\
.91 \\
16\end{array}$ & $\begin{array}{r}3.46 \\
1.71 \\
13\end{array}$ & $\begin{array}{r}3.93 \\
1.00 \\
14\end{array}$ & $\begin{array}{r}3.91 \\
1.23 \\
78\end{array}$ & 1.173 & .330 \\
\hline $\begin{array}{l}\text { 46. Manifest Christ-like } \\
\text { character in spiritual } \\
\text { leadership roles } \\
M \\
S D \\
N\end{array}$ & $\begin{array}{r}4.94 \\
.24 \\
18\end{array}$ & $\begin{array}{r}4.82 \\
.53 \\
17\end{array}$ & $\begin{array}{r}4.88 \\
.34 \\
16\end{array}$ & $\begin{array}{r}4.85 \\
.38 \\
13\end{array}$ & $\begin{array}{r}4.64 \\
.63 \\
14\end{array}$ & $\begin{array}{r}4.83 \\
.44 \\
78\end{array}$ & .988 & .420 \\
\hline
\end{tabular}


Table 46-Continued.

\begin{tabular}{|l|r|r|r|r|r|r|r|r|}
\hline Sub-Hypotheses & $\begin{array}{l}\text { Board } \\
\text { Chair }\end{array}$ & $\begin{array}{l}\text { Presi- } \\
\text { dent }\end{array}$ & $\begin{array}{l}\text { Aca- } \\
\text { demic } \\
\text { VP }\end{array}$ & $\begin{array}{c}\text { Student } \\
\text { Service } \\
\text { VP }\end{array}$ & $\begin{array}{c}\text { Finance } \\
\text { VP }\end{array}$ & Total & $F$ & $P$ \\
\hline 47. Focus on the & & & & & & & & \\
Christian philosophy & & & & & & & & \\
of the institution & & & & & & & & \\
$M$ & 4.94 & 4.94 & 4.88 & 4.92 & 4.71 & 4.88 & 1.343 & .262 \\
$S D$ & .24 & .24 & .34 & .28 & .47 & .32 & & \\
$N$ & 18 & 17 & 16 & 13 & 14 & 78 & & \\
\hline
\end{tabular}




\section{APPENDIX I}

TABLES $47-53$

COMPARATIVE ONE-WAY ANOVA RESULTS

FOR ACTUAL AND EXPECTED ROLES

FOR SUB-HYPOTHESES 1-47 
Table 47. Comparative one-way ANOVA results for sub-hypotheses 1-8 regarding presidents' actual and expected roles in student-related functions

\begin{tabular}{|c|c|c|c|c|c|c|c|}
\hline No. & Variable & $\begin{array}{l}\text { Source of } \\
\text { Variance }\end{array}$ & $\begin{array}{l}\text { Sum of } \\
\text { Squares }\end{array}$ & $d f$ & $\begin{array}{l}\text { Mean } \\
\text { Square }\end{array}$ & $\boldsymbol{F}$ & Sig. \\
\hline \multirow{2}{*}{1.} & $\begin{array}{l}\text { Participate in student } \\
\text { recruitment } \\
\text {-- Actual }\end{array}$ & $\begin{array}{l}\text { Between Groups } \\
\text { Within Groups } \\
\text { Total }\end{array}$ & $\begin{array}{r}10.410 \\
112.577 \\
122.987\end{array}$ & $\begin{array}{r}4 \\
73 \\
77\end{array}$ & $\begin{array}{l}2.602 \\
1.542\end{array}$ & 1.688 & .162 \\
\hline & $\begin{array}{l}\text { Participate in student } \\
\text { recruitment } \\
\text {-- Expected }\end{array}$ & $\begin{array}{l}\text { Between Groups } \\
\text { Within Groups } \\
\text { Total }\end{array}$ & $\begin{array}{l}11.438 \\
85.434 \\
96.872\end{array}$ & $\begin{array}{r}4 \\
73 \\
77\end{array}$ & $\begin{array}{l}2.859 \\
1.170\end{array}$ & 2.443 & .054 \\
\hline \multirow{2}{*}{2.} & $\begin{array}{l}\text { Work closely with student } \\
\text { government leaders } \\
-- \text { Actual }\end{array}$ & $\begin{array}{l}\text { Between Groups } \\
\text { Within Groups } \\
\text { Total }\end{array}$ & $\begin{array}{r}6.264 \\
1.9 .954 \\
116.218\end{array}$ & $\begin{array}{r}4 \\
73 \\
77\end{array}$ & $\begin{array}{l}1.566 \\
1.506\end{array}$ & 1.040 & .393 \\
\hline & $\begin{array}{l}\text { Work closely with student } \\
\text { government leaders } \\
\text {-- Expected }\end{array}$ & $\begin{array}{l}\text { Between Groups } \\
\text { Within Groups } \\
\text { Total }\end{array}$ & $\begin{array}{r}4.592 \\
95.062 \\
99.654\end{array}$ & $\begin{array}{r}4 \\
73 \\
77\end{array}$ & $\begin{array}{l}1.148 \\
1.302\end{array}$ & .881 & .479 \\
\hline \multirow{2}{*}{3.} & $\begin{array}{l}\text { Dialogue frequently with } \\
\text { students } \\
\text {-- Actual }\end{array}$ & $\begin{array}{l}\text { Between Groups } \\
\text { Within Groups } \\
\text { Total }\end{array}$ & $\begin{array}{r}8.731 \\
90.615 \\
99.346\end{array}$ & $\begin{array}{r}4 \\
73 \\
77\end{array}$ & $\begin{array}{l}2.183 \\
1.241\end{array}$ & 1.757 & .147 \\
\hline & $\begin{array}{l}\text { Dialogue frequently with } \\
\text { students } \\
\text {-- Expected }\end{array}$ & $\begin{array}{l}\text { Between Groups } \\
\text { Within Groups } \\
\text { Total }\end{array}$ & $\begin{array}{r}4.069 \\
54.047 \\
58.115\end{array}$ & $\begin{array}{r}4 \\
73 \\
77\end{array}$ & $\begin{array}{r}1.017 \\
.740\end{array}$ & 1.374 & .251 \\
\hline \multirow{2}{*}{4.} & $\begin{array}{l}\text { Visit students periodically } \\
\text { in dorms during dorm } \\
\text { worships } \\
- \text { - Actual }\end{array}$ & $\begin{array}{l}\text { Between Groups } \\
\text { Within Groups } \\
\text { Total }\end{array}$ & $\begin{array}{r}8.120 \\
111.175 \\
119.295\end{array}$ & $\begin{array}{r}4 \\
73 \\
77\end{array}$ & $\begin{array}{l}2.030 \\
1.523\end{array}$ & 1.333 & .266 \\
\hline & $\begin{array}{l}\text { Visit students periodically } \\
\text { in dorms during dorm } \\
\text { worships } \\
\text {-- Expected }\end{array}$ & $\begin{array}{l}\text { Between Groups } \\
\text { Within Groups } \\
\text { Total }\end{array}$ & $\begin{array}{r}8.214 \\
111.581 \\
119.795\end{array}$ & $\begin{array}{r}4 \\
73 \\
77\end{array}$ & $\begin{array}{l}2.054 \\
1.529\end{array}$ & 1.343 & .262 \\
\hline \multirow{2}{*}{5.} & $\begin{array}{l}\text { Take ultimate responsibility } \\
\text { for student discipline } \\
\text {-- Actual }\end{array}$ & $\begin{array}{l}\text { Between Groups } \\
\text { Within Groups } \\
\text { Total }\end{array}$ & $\begin{array}{r}14.887 \\
146.652 \\
161.538\end{array}$ & $\begin{array}{r}4 \\
73 \\
77\end{array}$ & $\begin{array}{l}3.722 \\
2.009\end{array}$ & 1.853 & .128 \\
\hline & $\begin{array}{l}\text { Take ultimate responsibility } \\
\text { for student discipline } \\
\text {-- Expected }\end{array}$ & $\begin{array}{l}\text { Between Groups } \\
\text { Within Groups } \\
\text { Total }\end{array}$ & $\begin{array}{r}19.347 \\
163.371 \\
182.718\end{array}$ & $\begin{array}{r}4 \\
73 \\
77\end{array}$ & $\begin{array}{l}4.837 \\
2.238\end{array}$ & 2.161 & .082 \\
\hline
\end{tabular}


Table 47--Continued.

\begin{tabular}{|c|c|c|c|c|c|c|c|}
\hline No. & Variable & $\begin{array}{l}\text { Source of } \\
\text { Variance }\end{array}$ & $\begin{array}{l}\text { Sum of } \\
\text { Squares }\end{array}$ & $d f$ & $\begin{array}{l}\text { Mean } \\
\text { Square }\end{array}$ & $F$ & Sig. \\
\hline \multirow{2}{*}{6.} & $\begin{array}{l}\text { Teach some classes to } \\
\text { maintain contact with } \\
\text { students } \\
\text {-- Actual }\end{array}$ & $\begin{array}{l}\text { Between Groups } \\
\text { Within Groups } \\
\text { Total }\end{array}$ & $\begin{array}{r}17.981 \\
167.314 \\
185.295\end{array}$ & $\begin{array}{r}4 \\
73 \\
77\end{array}$ & $\begin{array}{l}4.495 \\
2.292\end{array}$ & 1.961 & .109 \\
\hline & $\begin{array}{l}\text { Teach some classes to } \\
\text { maintain contact with } \\
\text { students } \\
\text {-- Expected }\end{array}$ & $\begin{array}{l}\text { Between Groups } \\
\text { Within Groups } \\
\text { Total }\end{array}$ & $\begin{array}{r}5.985 \\
171.362 \\
177.346\end{array}$ & $\begin{array}{r}4 \\
73 \\
77\end{array}$ & $\begin{array}{l}1.496 \\
2.347\end{array}$ & .637 & .637 \\
\hline \multirow{2}{*}{7.} & $\begin{array}{l}\text { Know every student } \\
\text { personally } \\
\text {-- Actual }\end{array}$ & $\begin{array}{l}\text { Between Groups } \\
\text { Within Groups } \\
\text { Total }\end{array}$ & $\begin{array}{r}5.742 \\
103.437 \\
109.179\end{array}$ & $\begin{array}{r}4 \\
73 \\
77\end{array}$ & $\begin{array}{l}1.436 \\
1.417\end{array}$ & 1.013 & .406 \\
\hline & $\begin{array}{l}\text { Know every student } \\
\text { personally } \\
\text {-- Expected }\end{array}$ & $\begin{array}{l}\text { Between Groups } \\
\text { Within Groups } \\
\text { Total }\end{array}$ & $\begin{array}{r}6.129 \\
128.743 \\
134.872\end{array}$ & $\begin{array}{r}4 \\
73 \\
77\end{array}$ & $\begin{array}{l}1.532 \\
1.764\end{array}$ & .869 & .487 \\
\hline \multirow{2}{*}{8.} & $\begin{array}{l}\text { Serve as mentor of } \\
\text { students } \\
\text {-- Actual }\end{array}$ & $\begin{array}{l}\text { Between Groups } \\
\text { Within Groups } \\
\text { Total }\end{array}$ & $\begin{array}{r}17.986 \\
106.386 \\
124.372\end{array}$ & $\begin{array}{r}4 \\
73 \\
77\end{array}$ & $\begin{array}{l}4.497 \\
1.457\end{array}$ & 3.085 & .021 \\
\hline & $\begin{array}{l}\text { Serve as mentor of } \\
\text { students } \\
\text {-- Expected }\end{array}$ & $\begin{array}{l}\text { Between Groups } \\
\text { Within Groups } \\
\text { Total }\end{array}$ & $\begin{array}{r}17.480 \\
107.853 \\
125.333\end{array}$ & $\begin{array}{r}4 \\
73 \\
77\end{array}$ & $\begin{array}{l}4.370 \\
1.477\end{array}$ & 2.958 & .025 \\
\hline
\end{tabular}


Table 48. Comparative one-way ANOVA results for sub-hypotheses 9-16 regarding presidents' actual and expected roles in employee-related functions

\begin{tabular}{|c|c|c|c|c|c|c|c|}
\hline No. & Variable & $\begin{array}{l}\text { Source of } \\
\text { Variance }\end{array}$ & $\begin{array}{c}\text { Sum of } \\
\text { Squares }\end{array}$ & $d f$ & $\begin{array}{l}\text { Mean } \\
\text { Square }\end{array}$ & $F$ & Sig. \\
\hline \multirow{2}{*}{9.} & $\begin{array}{l}\text { Participate in faculty } \\
\text { recruitment } \\
\text {-- Actual }\end{array}$ & $\begin{array}{l}\text { Between Groups } \\
\text { Within Groups } \\
\text { Total }\end{array}$ & $\begin{array}{r}7.821 \\
67.974 \\
75.795\end{array}$ & $\begin{array}{r}4 \\
73 \\
77\end{array}$ & $\begin{array}{r}1.955 \\
.931\end{array}$ & 2.100 & .090 \\
\hline & $\begin{array}{l}\text { Participate in faculty } \\
\text { recruitment } \\
\text {-- Expected }\end{array}$ & $\begin{array}{l}\text { Between Groups } \\
\text { Within Groups } \\
\text { Total }\end{array}$ & $\begin{array}{r}6.290 \\
63.005 \\
69.295\end{array}$ & $\begin{array}{r}4 \\
73 \\
77\end{array}$ & $\begin{array}{r}1.573 \\
.863\end{array}$ & 1.822 & .134 \\
\hline \multirow{2}{*}{10.} & $\begin{array}{l}\text { Serve as academic leader } \\
\text { of faculty } \\
\text {-- Actual }\end{array}$ & $\begin{array}{l}\text { Between Groups } \\
\text { Within Groups } \\
\text { Total }\end{array}$ & $\begin{array}{r}6.009 \\
115.940 \\
121.949\end{array}$ & $\begin{array}{r}4 \\
73 \\
77\end{array}$ & $\begin{array}{l}1.502 \\
1.588\end{array}$ & .946 & .443 \\
\hline & $\begin{array}{l}\text { Serve as academic leader } \\
\text { of faculty } \\
\text {-- Expected }\end{array}$ & $\begin{array}{l}\text { Between Groups } \\
\text { Within Groups } \\
\text { Total }\end{array}$ & $\begin{array}{r}3.930 \\
118.685 \\
122.615\end{array}$ & $\begin{array}{r}4 \\
73 \\
77\end{array}$ & $\begin{array}{r}.983 \\
1.626\end{array}$ & .604 & .661 \\
\hline \multirow{2}{*}{11.} & $\begin{array}{l}\text { Guard academic freedom } \\
\text { of faculty } \\
\text {-- Actual }\end{array}$ & $\begin{array}{l}\text { Between Groups } \\
\text { Within Groups } \\
\text { Total }\end{array}$ & $\begin{array}{r}7.941 \\
75.854 \\
83.795\end{array}$ & $\begin{array}{r}4 \\
73 \\
77\end{array}$ & $\begin{array}{l}1.985 \\
1.039\end{array}$ & 1.910 & .118 \\
\hline & $\begin{array}{l}\text { Guard academic freedom } \\
\text { of faculty } \\
\text {-- Expected }\end{array}$ & $\begin{array}{l}\text { Between Groups } \\
\text { Within Groups } \\
\text { Total }\end{array}$ & $\begin{array}{r}2.273 \\
55.689 \\
57.962\end{array}$ & $\begin{array}{r}4 \\
73 \\
77\end{array}$ & $\begin{array}{l}.568 \\
.763\end{array}$ & .745 & .565 \\
\hline \multirow{2}{*}{12.} & $\begin{array}{l}\text { Have responsibility in } \\
\text { submitting proposed } \\
\text { changes in academic } \\
\text { matters to faculty for } \\
\text { approval } \\
\text {-- Actual }\end{array}$ & $\begin{array}{l}\text { Between Groups } \\
\text { Within Groups } \\
\text { Total }\end{array}$ & $\begin{array}{r}19.641 \\
113.244 \\
132.885\end{array}$ & $\begin{array}{r}4 \\
73 \\
77\end{array}$ & $\begin{array}{l}4.910 \\
1.551\end{array}$ & 3.165 & .019 \\
\hline & $\begin{array}{l}\text { Have responsibility in } \\
\text { submitting proposed } \\
\text { changes in academic } \\
\text { matters to faculty for } \\
\text { approval } \\
\text {-- Expected }\end{array}$ & $\begin{array}{l}\text { Between Groups } \\
\text { Within Groups } \\
\text { Total }\end{array}$ & $\begin{array}{r}21.210 \\
125.508 \\
146.718\end{array}$ & $\begin{array}{r}4 \\
73 \\
77\end{array}$ & $\begin{array}{l}5.303 \\
1.719\end{array}$ & 3.084 & .021 \\
\hline
\end{tabular}


Table 48--Continued.

\begin{tabular}{|c|c|c|c|c|c|c|c|}
\hline No. & Variable & $\begin{array}{l}\text { Source of } \\
\text { Variance }\end{array}$ & $\begin{array}{l}\text { Sum of } \\
\text { Squares }\end{array}$ & $d f$ & $\begin{array}{l}\text { Mean } \\
\text { Square }\end{array}$ & $F$ & Sig. \\
\hline \multirow{2}{*}{13} & $\begin{array}{l}\text { Work with faculty in } \\
\text { reviewing accrediting } \\
\text { agency's reports } \\
\text { - Actual }\end{array}$ & $\begin{array}{l}\text { Between Groups } \\
\text { Within Groups } \\
\text { Total }\end{array}$ & $\begin{array}{r}13.415 \\
87.931 \\
101.346\end{array}$ & $\begin{array}{r}4 \\
73 \\
77\end{array}$ & $\begin{array}{l}3.354 \\
1.205\end{array}$ & 2.784 & .033 \\
\hline & $\begin{array}{l}\text { Work with faculty in } \\
\text { reviewing accrediting } \\
\text { agency's reports } \\
\text { - Expected }\end{array}$ & $\begin{array}{l}\text { Between Groups } \\
\text { Within Groups } \\
\text { Total }\end{array}$ & $\begin{array}{r}3.122 \\
74.724 \\
77.846\end{array}$ & $\begin{array}{r}4 \\
73 \\
77\end{array}$ & $\begin{array}{r}.780 \\
1.024\end{array}$ & .762 & .553 \\
\hline \multirow[t]{2}{*}{14.} & $\begin{array}{l}\text { Recommend to board for } \\
\text { hiring, promotion, } \\
\text { demotion, and dismissal } \\
\text { of faculty and staff } \\
\text {-- Actual }\end{array}$ & $\begin{array}{l}\text { Between Groups } \\
\text { Within Groups } \\
\text { Total }\end{array}$ & $\begin{array}{r}3.599 \\
96.555 \\
100.154\end{array}$ & $\begin{array}{r}4 \\
73 \\
77\end{array}$ & $\begin{array}{r}.900 \\
1.323\end{array}$ & .680 & .608 \\
\hline & $\begin{array}{l}\text { Recommend to board for } \\
\text { hiring, promotion, } \\
\text { demotion, and dismissal } \\
\text { of faculty and staff } \\
\text {-- Expected }\end{array}$ & $\begin{array}{l}\text { Between Groups } \\
\text { Within Groups } \\
\text { Total }\end{array}$ & $\begin{array}{r}3.118 \\
71.754 \\
74.872\end{array}$ & $\begin{array}{r}4 \\
73 \\
77\end{array}$ & $\begin{array}{l}.779 \\
.983\end{array}$ & .793 & .534 \\
\hline \multirow{2}{*}{15.} & $\begin{array}{l}\text { Collaborate for advanced } \\
\text { in-service training for } \\
\text { faculty and staff } \\
\text { - Actual }\end{array}$ & $\begin{array}{l}\text { Between Groups } \\
\text { Within Groups } \\
\text { Total }\end{array}$ & $\begin{array}{r}17.411 \\
103.627 \\
121.038\end{array}$ & $\begin{array}{r}4 \\
73 \\
77\end{array}$ & $\begin{array}{l}4.353 \\
1.420\end{array}$ & 3.066 & .022 \\
\hline & $\begin{array}{l}\text { Collaborate for advanced } \\
\text { in-service training for } \\
\text { faculty and staff } \\
\text {-- Expected }\end{array}$ & $\begin{array}{l}\text { Between Groups } \\
\text { Within Groups } \\
\text { Total }\end{array}$ & $\begin{array}{r}13.642 \\
102.012 \\
115.654\end{array}$ & $\begin{array}{r}4 \\
73 \\
77\end{array}$ & $\begin{array}{l}3.411 \\
1.397\end{array}$ & 2.441 & .054 \\
\hline \multirow{2}{*}{16.} & $\begin{array}{l}\text { Carry out policies and } \\
\text { regulations of faculty and } \\
\text { staff } \\
\text {-- Actual }\end{array}$ & $\begin{array}{l}\text { Between Groups } \\
\text { Within Groups } \\
\text { Total }\end{array}$ & $\begin{array}{r}6.964 \\
131.497 \\
138.462\end{array}$ & $\begin{array}{r}4 \\
73 \\
77\end{array}$ & $\begin{array}{l}1.741 \\
1.801\end{array}$ & .967 & .431 \\
\hline & $\begin{array}{l}\text { Carry out policies and } \\
\text { regulations of faculty and } \\
\text { staff } \\
\text {-- Expected }\end{array}$ & $\begin{array}{l}\text { Between Groups } \\
\text { Within Groups } \\
\text { Total }\end{array}$ & $\begin{array}{r}3.109 \\
120.071 \\
123.179\end{array}$ & $\begin{array}{r}4 \\
73 \\
77\end{array}$ & $\begin{array}{r}.777 \\
1.645\end{array}$ & .473 & .756 \\
\hline
\end{tabular}


Table 49. Comparative one-way ANOVA results for sub-hypotheses 17-25 regarding presidents' actual and expected roles in general administrative functions

\begin{tabular}{|c|c|c|c|c|c|c|c|}
\hline No. & Variable & $\begin{array}{l}\text { Source of } \\
\text { Variance }\end{array}$ & $\begin{array}{c}\text { Sum of } \\
\text { Squares }\end{array}$ & $d f$ & $\begin{array}{c}\text { Mean } \\
\text { Square }\end{array}$ & $F$ & Sig. \\
\hline \multirow{2}{*}{17.} & $\begin{array}{l}\text { Prepare agenda for board } \\
\text { of trustees' meetings } \\
\text {-- Actual }\end{array}$ & $\begin{array}{l}\text { Between Groups } \\
\text { Within Groups } \\
\text { Total }\end{array}$ & $\begin{array}{r}4.289 \\
43.659 \\
47.949\end{array}$ & $\begin{array}{r}4 \\
73 \\
77\end{array}$ & $\begin{array}{r}1.072 \\
.598\end{array}$ & 1.793 & .139 \\
\hline & $\begin{array}{l}\text { Prepare agenda for board } \\
\text { of trustees' meetings } \\
\text {-- Expected }\end{array}$ & $\begin{array}{l}\text { Between Groups } \\
\text { Within Groups } \\
\text { Total }\end{array}$ & $\begin{array}{r}1.831 \\
26.285 \\
28.115\end{array}$ & $\begin{array}{r}4 \\
73 \\
77\end{array}$ & $\begin{array}{l}.458 \\
.360\end{array}$ & 1.271 & .289 \\
\hline \multirow{2}{*}{18.} & $\begin{array}{l}\text { Preside over general } \\
\text { faculty meetings } \\
\text {-- Actual }\end{array}$ & $\begin{array}{l}\text { Between Groups } \\
\text { Within Groups } \\
\text { Total }\end{array}$ & $\begin{array}{r}11.126 \\
123.862 \\
134.987\end{array}$ & $\begin{array}{r}4 \\
73 \\
77\end{array}$ & $\begin{array}{l}2.781 \\
1.697\end{array}$ & 1.639 & .174 \\
\hline & $\begin{array}{l}\text { Preside over general } \\
\text { faculty meetings } \\
\text {-- Expected }\end{array}$ & $\begin{array}{l}\text { Between Groups } \\
\text { Within Groups } \\
\text { Total }\end{array}$ & $\begin{array}{r}10.439 \\
129.510 \\
139.949\end{array}$ & $\begin{array}{r}4 \\
73 \\
77\end{array}$ & $\begin{array}{l}2.610 \\
1.774\end{array}$ & 1.471 & .220 \\
\hline \multirow{2}{*}{19.} & $\begin{array}{l}\text { Take responsibility in } \\
\text { structuring student } \\
\text { admission standards } \\
\text {-- Actual }\end{array}$ & $\begin{array}{l}\text { Between Groups } \\
\text { Within Groups } \\
\text { Total }\end{array}$ & $\begin{array}{r}15.075 \\
96.874 \\
111.949\end{array}$ & $\begin{array}{r}4 \\
73 \\
77\end{array}$ & $\begin{array}{l}3.769 \\
1.327\end{array}$ & 2.840 & .030 \\
\hline & $\begin{array}{l}\text { Take responsibility in } \\
\text { structuring student } \\
\text { admission standards } \\
\text {-- Expected }\end{array}$ & $\begin{array}{l}\text { Between Groups } \\
\text { Within Groups } \\
\text { Total }\end{array}$ & $\begin{array}{r}17.385 \\
103.961 \\
121.346\end{array}$ & $\begin{array}{r}4 \\
73 \\
77\end{array}$ & $\begin{array}{l}4.346 \\
1.424\end{array}$ & 3.052 & .022 \\
\hline \multirow{2}{*}{20.} & $\begin{array}{l}\text { Take responsibility in } \\
\text { strategic planning for } \\
\text { institution } \\
\text {-- Actual }\end{array}$ & $\begin{array}{l}\text { Between Groups } \\
\text { Within Groups } \\
\text { Total }\end{array}$ & $\begin{array}{r}4.441 \\
79.777 \\
84.218\end{array}$ & $\begin{array}{r}4 \\
73 \\
77\end{array}$ & $\begin{array}{l}1.110 \\
1.093\end{array}$ & 1.016 & .405 \\
\hline & $\begin{array}{l}\text { Take responsibility in } \\
\text { strategic planning for } \\
\text { institution } \\
\text {-- Expected }\end{array}$ & $\begin{array}{l}\text { Between Groups } \\
\text { Within Groups } \\
\text { Total }\end{array}$ & $\begin{array}{r}.878 \\
27.494 \\
28.372\end{array}$ & $\begin{array}{r}4 \\
73 \\
77\end{array}$ & $\begin{array}{l}.219 \\
.377\end{array}$ & .583 & .676 \\
\hline
\end{tabular}


Table 49-Continued.

\begin{tabular}{|c|c|c|c|c|c|c|c|}
\hline No. & Variable & $\begin{array}{l}\text { Source of } \\
\text { Variance }\end{array}$ & $\begin{array}{l}\text { Sum of } \\
\text { Squares }\end{array}$ & $d f$ & $\begin{array}{l}\text { Mean } \\
\text { Square }\end{array}$ & $F$ & Sig. \\
\hline \multirow{2}{*}{21.} & $\begin{array}{l}\text { Take a leading role to } \\
\text { prepare, revise, and } \\
\text { follow organizational } \\
\text { chart -- Actual }\end{array}$ & $\begin{array}{l}\text { Between Groups } \\
\text { Within Groups } \\
\text { Total }\end{array}$ & $\begin{array}{r}7.985 \\
86.900 \\
94.885\end{array}$ & $\begin{array}{r}4 \\
73 \\
77\end{array}$ & $\begin{array}{l}1.996 \\
1.190\end{array}$ & 1.677 & .165 \\
\hline & $\begin{array}{l}\text { Take a leading role to } \\
\text { prepare, revise, and } \\
\text { follow organizational } \\
\text { chart -- Expected }\end{array}$ & $\begin{array}{l}\text { Between Groups } \\
\text { Within Groups } \\
\text { Total }\end{array}$ & $\begin{array}{r}2.195 \\
41.254 \\
43.449\end{array}$ & $\begin{array}{r}4 \\
73 \\
77\end{array}$ & $\begin{array}{l}.549 \\
.565\end{array}$ & .971 & .429 \\
\hline \multirow{2}{*}{22.} & $\begin{array}{l}\text { Foster research and } \\
\text { intellectual climate } \\
\text {-- Actual }\end{array}$ & $\begin{array}{l}\text { Between Groups } \\
\text { Within Groups } \\
\text { Total }\end{array}$ & $\begin{array}{r}4.652 \\
93.464 \\
98.115\end{array}$ & $\begin{array}{r}4 \\
73 \\
77\end{array}$ & $\begin{array}{l}1.163 \\
1.280\end{array}$ & .908 & .464 \\
\hline & $\begin{array}{l}\text { Foster research and } \\
\text { intellectual climate } \\
\text {-- Expected }\end{array}$ & $\begin{array}{l}\text { Between Groups } \\
\text { Within Groups } \\
\text { Total }\end{array}$ & $\begin{array}{r}1.493 \\
51.853 \\
53.346\end{array}$ & $\begin{array}{r}4 \\
73 \\
77\end{array}$ & $\begin{array}{l}.373 \\
.710\end{array}$ & .525 & .717 \\
\hline \multirow{2}{*}{23.} & $\begin{array}{l}\text { Comply with legal } \\
\text { mandates and laws in } \\
\text { addressing grievances } \\
\text {-- Actual }\end{array}$ & $\begin{array}{l}\text { Between Groups } \\
\text { Within Groups } \\
\text { Total }\end{array}$ & $\begin{array}{r}5.267 \\
61.605 \\
66.872\end{array}$ & $\begin{array}{r}4 \\
73 \\
77\end{array}$ & $\begin{array}{r}1.317 \\
.844\end{array}$ & 1.560 & .194 \\
\hline & $\begin{array}{l}\text { Comply with legal } \\
\text { mandates and laws in } \\
\text { addressing grievances } \\
\text {-- Expected }\end{array}$ & $\begin{array}{l}\text { Between Groups } \\
\text { Within Groups } \\
\text { Total }\end{array}$ & $\begin{array}{r}2.695 \\
39.985 \\
42.679\end{array}$ & $\begin{array}{r}4 \\
73 \\
77\end{array}$ & $\begin{array}{l}.674 \\
.548\end{array}$ & 1.230 & .306 \\
\hline \multirow[t]{2}{*}{24.} & $\begin{array}{l}\text { Help formulate and } \\
\text { adhere to educational } \\
\text { philosophy } \\
\text {-- Actual }\end{array}$ & $\begin{array}{l}\text { Between Groups } \\
\text { Within Groups } \\
\text { Total }\end{array}$ & $\begin{array}{r}9.750 \\
55.429 \\
65.179\end{array}$ & $\begin{array}{r}4 \\
73 \\
77\end{array}$ & $\begin{array}{r}2.438 \\
.759\end{array}$ & 3.210 & .017 \\
\hline & $\begin{array}{l}\text { Help formulate and } \\
\text { adhere to educational } \\
\text { philosophy } \\
\text {-- Expected }\end{array}$ & $\begin{array}{l}\text { Between Groups } \\
\text { Within Groups } \\
\text { Total }\end{array}$ & $\begin{array}{r}.629 \\
10.089 \\
10.718\end{array}$ & $\begin{array}{r}4 \\
73 \\
77\end{array}$ & $\begin{array}{l}.157 \\
.138\end{array}$ & 1.138 & .346 \\
\hline \multirow{2}{*}{25.} & $\begin{array}{l}\text { Delegate responsibility, } \\
\text { authority, and needed } \\
\text { resources to appropriate } \\
\text { administrators -- Actual }\end{array}$ & $\begin{array}{l}\text { Between Groups } \\
\text { Within Groups } \\
\text { Total }\end{array}$ & $\begin{array}{r}7.771 \\
91.678 \\
99.449\end{array}$ & $\begin{array}{r}4 \\
73 \\
77\end{array}$ & $\begin{array}{l}1.943 \\
1.256\end{array}$ & 1.547 & .198 \\
\hline & $\begin{array}{l}\text { Delegate responsibility, } \\
\text { authority, and needed } \\
\text { resources to appropriate } \\
\text { administrators - } \\
\text { Expected }\end{array}$ & $\begin{array}{l}\text { Between Groups } \\
\text { Within Groups } \\
\text { Total }\end{array}$ & $\begin{array}{r}2.356 \\
18.990 \\
21.346\end{array}$ & $\begin{array}{r}4 \\
73 \\
77\end{array}$ & $\begin{array}{l}.589 \\
.260\end{array}$ & 2.264 & .070 \\
\hline
\end{tabular}


Table 50. Comparative one-way ANOVA results for sub-hypotheses 26-30 regarding presidents' actual and expected roles in finance-related functions

\begin{tabular}{|c|c|c|c|c|c|c|c|}
\hline No. & Variable & $\begin{array}{l}\text { Source of } \\
\text { Variance }\end{array}$ & $\begin{array}{c}\text { Sum of } \\
\text { Squares }\end{array}$ & $d f$ & $\begin{array}{c}\text { Mean } \\
\text { Square }\end{array}$ & $F$ & Sig. \\
\hline \multirow{2}{*}{26.} & $\begin{array}{l}\text { Participate in the prepara- } \\
\text { tion of annual budget of } \\
\text { institution -- Actual }\end{array}$ & $\begin{array}{l}\text { Between Groups } \\
\text { Within Groups } \\
\text { Total }\end{array}$ & $\begin{array}{r}8.273 \\
119.266 \\
127.538\end{array}$ & $\begin{array}{r}4 \\
73 \\
77\end{array}$ & $\begin{array}{l}2.068 \\
1.634\end{array}$ & 1.266 & .291 \\
\hline & $\begin{array}{l}\text { Participate in the prepara- } \\
\text { tion of annual budget of } \\
\text { institution -- Expected }\end{array}$ & $\begin{array}{l}\text { Between Groups } \\
\text { Within Groups } \\
\text { Total }\end{array}$ & $\begin{array}{l}10.388 \\
54.291 \\
64.679\end{array}$ & $\begin{array}{r}4 \\
73 \\
77\end{array}$ & $\begin{array}{r}2.597 \\
.744\end{array}$ & 3.492 & .012 \\
\hline \multirow{2}{*}{27.} & $\begin{array}{l}\text { Ensure that institution's } \\
\text { budget, after approval by } \\
\text { board, is adhered to } \\
\text {-- Actual }\end{array}$ & $\begin{array}{l}\text { Between Groups } \\
\text { Within Groups } \\
\text { Total }\end{array}$ & $\begin{array}{r}2.261 \\
109.085 \\
111.346\end{array}$ & $\begin{array}{r}4 \\
73 \\
77\end{array}$ & $\begin{array}{r}.565 \\
1.494\end{array}$ & .378 & .823 \\
\hline & $\begin{array}{l}\text { Ensure that institution's } \\
\text { budget, after approval by } \\
\text { board, is adhered to } \\
\text {-- Expected }\end{array}$ & $\begin{array}{l}\text { Between Groups } \\
\text { Within Groups } \\
\text { Total }\end{array}$ & $\begin{array}{r}3.364 \\
55.930 \\
59.295\end{array}$ & $\begin{array}{r}4 \\
73 \\
77\end{array}$ & $\begin{array}{l}.841 \\
.766\end{array}$ & 1.098 & .364 \\
\hline \multirow{2}{*}{28.} & $\begin{array}{l}\text { Present annual audited } \\
\text { financial statement to board } \\
\text {-- Actual }\end{array}$ & $\begin{array}{l}\text { Between Groups } \\
\text { Within Groups } \\
\text { Total }\end{array}$ & $\begin{array}{r}4.007 \\
161.954 \\
165.962\end{array}$ & $\begin{array}{r}4 \\
73 \\
77\end{array}$ & $\begin{array}{l}1.002 \\
2.219\end{array}$ & .452 & .771 \\
\hline & $\begin{array}{l}\text { Present annual audited } \\
\text { financial statement to board } \\
\text {-- Expected }\end{array}$ & $\begin{array}{l}\text { Between Groups } \\
\text { Within Groups } \\
\text { Total }\end{array}$ & $\begin{array}{r}9.688 \\
164.774 \\
174.462\end{array}$ & $\begin{array}{r}4 \\
73 \\
77\end{array}$ & $\begin{array}{l}2.422 \\
2.257\end{array}$ & 1.073 & .376 \\
\hline \multirow{2}{*}{29.} & $\begin{array}{l}\text { Recommend to board the } \\
\text { salary changes for faculty } \\
\text { and staff positions } \\
\text {-- Actual }\end{array}$ & $\begin{array}{l}\text { Between Groups } \\
\text { Within Groups } \\
\text { Total }\end{array}$ & $\begin{array}{r}11.709 \\
144.086 \\
155.795\end{array}$ & $\begin{array}{r}4 \\
73 \\
77\end{array}$ & $\begin{array}{l}2.927 \\
1.974\end{array}$ & 1.483 & .216 \\
\hline & $\begin{array}{l}\text { Recommend to board the } \\
\text { salary changes for faculty } \\
\text { and staff positions } \\
\text {-- Expected }\end{array}$ & $\begin{array}{l}\text { Between Groups } \\
\text { Within Groups } \\
\text { Total }\end{array}$ & $\begin{array}{r}10.768 \\
139.847 \\
140.615\end{array}$ & $\begin{array}{r}4 \\
73 \\
77\end{array}$ & $\begin{array}{l}2.692 \\
1.916\end{array}$ & 1.405 & .241 \\
\hline \multirow{2}{*}{30.} & $\begin{array}{l}\text { Accept accountability to } \\
\text { board for financial } \\
\text { operation -- Actual }\end{array}$ & $\begin{array}{l}\text { Between Groups } \\
\text { Within Groups } \\
\text { Total }\end{array}$ & $\begin{array}{r}4.379 \\
95.416 \\
99.795\end{array}$ & $\begin{array}{r}4 \\
73 \\
77\end{array}$ & $\begin{array}{l}1.095 \\
1.307\end{array}$ & .838 & .506 \\
\hline & $\begin{array}{l}\text { Accept accountability to } \\
\text { board for financial } \\
\text { operation - Expected }\end{array}$ & $\begin{array}{l}\text { Between Groups } \\
\text { Within Groups } \\
\text { Total }\end{array}$ & $\begin{array}{r}2.172 \\
52.289 \\
54.462\end{array}$ & $\begin{array}{r}4 \\
73 \\
77\end{array}$ & $\begin{array}{l}.543 \\
.716\end{array}$ & .758 & .556 \\
\hline
\end{tabular}


Table 51. Comparative one-way ANOVA results for sub-hypotheses 31-36 regarding presidents' actual and expected roles in physical facilities-related functions

\begin{tabular}{|c|c|c|c|c|c|c|c|}
\hline No. & Variable & $\begin{array}{l}\text { Source of } \\
\text { Variance }\end{array}$ & $\begin{array}{c}\text { Sum of } \\
\text { Squares }\end{array}$ & $d f$ & $\begin{array}{l}\text { Mean } \\
\text { Square }\end{array}$ & $F$ & Sig. \\
\hline \multirow{2}{*}{31.} & $\begin{array}{l}\text { Present to board physical } \\
\text { needs of institution } \\
\text {-- Actual }\end{array}$ & $\begin{array}{l}\text { Between Groups } \\
\text { Within Groups } \\
\text { Total }\end{array}$ & $\begin{array}{r}14.041 \\
104.639 \\
118.679\end{array}$ & $\begin{array}{r}4 \\
73 \\
77\end{array}$ & $\begin{array}{l}3.510 \\
1.433\end{array}$ & 2.449 & .054 \\
\hline & $\begin{array}{l}\text { Present to board physical } \\
\text { needs of institution } \\
\text {-- Expected }\end{array}$ & $\begin{array}{l}\text { Between Groups } \\
\text { Within Groups } \\
\text { Total }\end{array}$ & $\begin{array}{r}15.429 \\
87.904 \\
103.333\end{array}$ & $\begin{array}{r}4 \\
73 \\
77\end{array}$ & $\begin{array}{l}3.857 \\
1.204\end{array}$ & 3.203 & .018 \\
\hline \multirow{2}{*}{32.} & $\begin{array}{l}\text { Direct the preparation of } \\
\text { new building plans } \\
\text {-- Actual }\end{array}$ & $\begin{array}{l}\text { Between Groups } \\
\text { Within Groups } \\
\text { Total }\end{array}$ & $\begin{array}{r}13.648 \\
145.852 \\
159.500\end{array}$ & $\begin{array}{r}4 \\
73 \\
77\end{array}$ & $\begin{array}{l}3.412 \\
1.998\end{array}$ & 1.708 & .158 \\
\hline & $\begin{array}{l}\text { Direct the preparation of } \\
\text { new building plans } \\
\text {-- Expected }\end{array}$ & $\begin{array}{l}\text { Between Groups } \\
\text { Within Groups } \\
\text { Total }\end{array}$ & $\begin{array}{r}16.456 \\
98.261 \\
114.718\end{array}$ & $\begin{array}{r}4 \\
73 \\
77\end{array}$ & $\begin{array}{l}4.114 \\
1.346\end{array}$ & 3.056 & .022 \\
\hline \multirow{2}{*}{33.} & $\begin{array}{l}\text { Plan for major repairs and } \\
\text { alterations } \\
\text {-- Actual }\end{array}$ & $\begin{array}{l}\text { Between Groups } \\
\text { Within Groups } \\
\text { Total }\end{array}$ & $\begin{array}{r}8.946 \\
138.849 \\
147.795\end{array}$ & $\begin{array}{r}4 \\
73 \\
77\end{array}$ & $\begin{array}{l}2.237 \\
1.902\end{array}$ & 1.176 & .329 \\
\hline & $\begin{array}{l}\text { Plan for major repairs and } \\
\text { alterations } \\
\text { - Expected }\end{array}$ & $\begin{array}{l}\text { Between Groups } \\
\text { Within Groups } \\
\text { Total }\end{array}$ & $\begin{array}{r}12.426 \\
126.408 \\
138.833\end{array}$ & $\begin{array}{r}4 \\
73 \\
77\end{array}$ & $\begin{array}{l}3.106 \\
1.732\end{array}$ & 1.794 & .139 \\
\hline \multirow{2}{*}{34.} & $\begin{array}{l}\text { Recommend to board for } \\
\text { equipment acquisition } \\
\text {-- Actual }\end{array}$ & $\begin{array}{l}\text { Between Groups } \\
\text { Within Groups } \\
\text { Total }\end{array}$ & $\begin{array}{r}8.103 \\
165.692 \\
173.795\end{array}$ & $\begin{array}{r}4 \\
73 \\
77\end{array}$ & $\begin{array}{l}2.026 \\
2.270\end{array}$ & .892 & .473 \\
\hline & $\begin{array}{l}\text { Recommend to board for } \\
\text { equipment acquisition } \\
\text {-- Expected }\end{array}$ & $\begin{array}{l}\text { Between Groups } \\
\text { Within Groups } \\
\text { Total }\end{array}$ & $\begin{array}{r}9.750 \\
174.199 \\
183.949 \\
\end{array}$ & $\begin{array}{r}4 \\
73 \\
77 \\
\end{array}$ & $\begin{array}{l}2.437 \\
2.386\end{array}$ & 1.021 & .402 \\
\hline \multirow{2}{*}{35.} & $\begin{array}{l}\text { Follow physical plant's } \\
\text { current master plan } \\
\text {-- Actual }\end{array}$ & $\begin{array}{l}\text { Between Groups } \\
\text { Within Groups } \\
\text { Total }\end{array}$ & $\begin{array}{r}11.346 \\
130.872 \\
142.218\end{array}$ & $\begin{array}{r}4 \\
73 \\
77\end{array}$ & $\begin{array}{l}2.837 \\
1.793\end{array}$ & 1.582 & .188 \\
\hline & $\begin{array}{l}\text { Follow physical plant's } \\
\text { current master plan } \\
\text {-- Expected }\end{array}$ & $\begin{array}{l}\text { Between Groups } \\
\text { Within Groups } \\
\text { Total }\end{array}$ & $\begin{array}{r}3.652 \\
86.720 \\
90.372\end{array}$ & $\begin{array}{r}4 \\
73 \\
77\end{array}$ & $\begin{array}{r}.913 \\
1.188\end{array}$ & .769 & .549 \\
\hline \multirow{2}{*}{36.} & $\begin{array}{l}\text { Ascertain that the campus } \\
\text { grounds are well kept } \\
\text { through appropriate } \\
\text { officers -- Actual }\end{array}$ & $\begin{array}{l}\text { Between Groups } \\
\text { Within Groups } \\
\text { Total }\end{array}$ & $\begin{array}{r}5.843 \\
101.503 \\
107.346\end{array}$ & $\begin{array}{r}4 \\
73 \\
77\end{array}$ & $\begin{array}{l}1.461 \\
1.390\end{array}$ & 1.051 & .387 \\
\hline & $\begin{array}{l}\text { Ascertain that the campus } \\
\text { grounds are well kept } \\
\text { through appropriate } \\
\text { officers -- Expected }\end{array}$ & $\begin{array}{l}\text { Between Groups } \\
\text { Within Groups } \\
\text { Total }\end{array}$ & $\begin{array}{r}3.355 \\
93.529 \\
96.885\end{array}$ & $\begin{array}{r}4 \\
73 \\
77\end{array}$ & $\begin{array}{r}.839 \\
1.281\end{array}$ & .655 & .625 \\
\hline
\end{tabular}


Table 52. Comparative one-way ANOVA results for sub-hypotheses 37-42 regarding presidents' actual and expected roles community- and alumni-related functions

\begin{tabular}{|c|c|c|c|c|c|c|c|}
\hline No. & Variable & $\begin{array}{l}\text { Source of } \\
\text { Variance }\end{array}$ & $\begin{array}{l}\text { Sum of } \\
\text { Squares }\end{array}$ & $d f$ & $\begin{array}{c}\text { Mean } \\
\text { Square }\end{array}$ & $F$ & Sig. \\
\hline \multirow{2}{*}{37.} & $\begin{array}{l}\text { Assist in fund-raising for } \\
\text { support of institution } \\
\text {-- Actual }\end{array}$ & $\begin{array}{l}\text { Between Groups } \\
\text { Within Groups } \\
\text { Total }\end{array}$ & $\begin{array}{r}1.733 \\
100.639 \\
102.372\end{array}$ & $\begin{array}{r}4 \\
73 \\
77\end{array}$ & $\begin{array}{r}.433 \\
1.379\end{array}$ & .314 & .868 \\
\hline & $\begin{array}{l}\text { Assist in fund-raising for } \\
\text { support of institution } \\
\text {-- Expected }\end{array}$ & $\begin{array}{l}\text { Between Groups } \\
\text { Within Groups } \\
\text { Total }\end{array}$ & $\begin{array}{r}.750 \\
31.096 \\
31.846\end{array}$ & $\begin{array}{r}4 \\
73 \\
77\end{array}$ & $\begin{array}{l}.188 \\
.426\end{array}$ & .440 & .779 \\
\hline \multirow{2}{*}{38.} & $\begin{array}{l}\text { Represent institution to } \\
\text { the churches of the } \\
\text { constituency } \\
\text {-- Actual }\end{array}$ & $\begin{array}{l}\text { Between Groups } \\
\text { Within Groups } \\
\text { Total }\end{array}$ & $\begin{array}{r}9.240 \\
55.440 \\
64.679\end{array}$ & $\begin{array}{r}4 \\
73 \\
77\end{array}$ & $\begin{array}{r}2.310 \\
.759\end{array}$ & 3.042 & .022 \\
\hline & $\begin{array}{l}\text { Represent institution to } \\
\text { the churches of the } \\
\text { constituency } \\
\text {-- Expected }\end{array}$ & $\begin{array}{l}\text { Between Groups } \\
\text { Within Groups } \\
\text { Total }\end{array}$ & $\begin{array}{r}1.140 \\
11.014 \\
12.154\end{array}$ & $\begin{array}{r}4 \\
73 \\
77\end{array}$ & $\begin{array}{l}.285 \\
.151\end{array}$ & 1.889 & .121 \\
\hline \multirow{2}{*}{39.} & $\begin{array}{l}\text { Present reports about } \\
\text { institution to community } \\
\text { and alumni } \\
\text {-- Actual }\end{array}$ & $\begin{array}{l}\text { Between Groups } \\
\text { Within Groups } \\
\text { Total }\end{array}$ & $\begin{array}{l}15.252 \\
72.543 \\
87.795\end{array}$ & $\begin{array}{r}4 \\
73 \\
77\end{array}$ & $\begin{array}{r}3.813 \\
.994\end{array}$ & 3.837 & .007 \\
\hline & $\begin{array}{l}\text { Present reports about } \\
\text { institution to community } \\
\text { and alumni } \\
\text {-- Expected }\end{array}$ & $\begin{array}{l}\text { Between Groups } \\
\text { Within Groups } \\
\text { Total }\end{array}$ & $\begin{array}{r}3.952 \\
27.381 \\
31.333\end{array}$ & $\begin{array}{r}4 \\
73 \\
77\end{array}$ & $\begin{array}{l}.988 \\
.375\end{array}$ & 2.634 & .041 \\
\hline \multirow{2}{*}{40.} & $\begin{array}{l}\text { Represent institution as } \\
\text { its official spokesperson } \\
\text { to public } \\
\text {-- Actual }\end{array}$ & $\begin{array}{l}\text { Between Groups } \\
\text { Within Groups } \\
\text { Total }\end{array}$ & $\begin{array}{r}4.977 \\
54.510 \\
59.487\end{array}$ & $\begin{array}{r}4 \\
73 \\
77\end{array}$ & $\begin{array}{r}1.244 \\
.747\end{array}$ & 1.666 & .167 \\
\hline & $\begin{array}{l}\text { Represent institution as } \\
\text { its official spokesperson } \\
\text { to public } \\
\text {-- Expected }\end{array}$ & $\begin{array}{l}\text { Between Groups } \\
\text { Within Groups } \\
\text { Total }\end{array}$ & $\begin{array}{r}1.529 \\
36.843 \\
38.372\end{array}$ & $\begin{array}{r}4 \\
73 \\
77\end{array}$ & $\begin{array}{l}.382 \\
.505\end{array}$ & .757 & .556 \\
\hline
\end{tabular}


Table 52--Continued.

\begin{tabular}{|c|c|c|c|c|c|c|c|}
\hline No. & Variable & $\begin{array}{l}\text { Source of } \\
\text { Variance }\end{array}$ & $\begin{array}{l}\text { Sum of } \\
\text { Squares }\end{array}$ & $d f$ & $\begin{array}{l}\text { Mean } \\
\text { Square }\end{array}$ & $F$ & Sig. \\
\hline \multirow{2}{*}{41.} & $\begin{array}{l}\text { Associate closely with } \\
\text { alumni groups } \\
\text { - Actual }\end{array}$ & $\begin{array}{l}\text { Between Groups } \\
\text { Within Groups } \\
\text { Total }\end{array}$ & $\begin{array}{r}4.426 \\
73.369 \\
77.795\end{array}$ & $\begin{array}{r}4 \\
73 \\
77\end{array}$ & $\begin{array}{l}1.106 \\
1.005\end{array}$ & 1.101 & .363 \\
\hline & $\begin{array}{l}\text { Associate closely with } \\
\text { alumni groups } \\
\text {-- Expected }\end{array}$ & $\begin{array}{l}\text { Between Groups } \\
\text { Within Groups } \\
\text { Total }\end{array}$ & $\begin{array}{r}.211 \\
29.277 \\
29.487\end{array}$ & $\begin{array}{r}4 \\
73 \\
77\end{array}$ & $\begin{array}{l}.053 \\
.401\end{array}$ & .131 & .970 \\
\hline \multirow{2}{*}{42.} & $\begin{array}{l}\text { Listen to constituency and } \\
\text { alumni } \\
\text {-- Actual }\end{array}$ & $\begin{array}{l}\text { Between Groups } \\
\text { Within Groups } \\
\text { Total }\end{array}$ & $\begin{array}{r}7.208 \\
76.639 \\
83.846\end{array}$ & $\begin{array}{r}4 \\
73 \\
77\end{array}$ & $\begin{array}{l}1.802 \\
1.050\end{array}$ & 1.716 & .156 \\
\hline & $\begin{array}{l}\text { Listen to constituency and } \\
\text { alumni } \\
\text {-- Expected }\end{array}$ & $\begin{array}{l}\text { Between Groups } \\
\text { Within Groups } \\
\text { Total }\end{array}$ & $\begin{array}{r}1.877 \\
14.995 \\
16.872\end{array}$ & $\begin{array}{r}4 \\
73 \\
77\end{array}$ & $\begin{array}{l}.469 \\
.205\end{array}$ & 2.285 & .068 \\
\hline
\end{tabular}


Table 53. Comparative one-way ANOVA results for sub-hypotheses 43-47 regarding presidents' actual and expected roles in spiritual life-related functions

\begin{tabular}{|c|c|c|c|c|c|c|c|}
\hline No. & Variable & $\begin{array}{l}\text { Source of } \\
\text { Variance }\end{array}$ & $\begin{array}{l}\text { Sum of } \\
\text { Squares }\end{array}$ & $d f$ & $\begin{array}{l}\text { Mean } \\
\text { Square }\end{array}$ & $F$ & Sig. \\
\hline \multirow{2}{*}{43.} & $\begin{array}{l}\text { Assume primary responsi- } \\
\text { bility for promotion of } \\
\text { religious life of students } \\
\text { at the institution via } \\
\text { delegation -- Actual }\end{array}$ & $\begin{array}{l}\text { Between Groups } \\
\text { Within Groups } \\
\text { Total }\end{array}$ & $\begin{array}{l}10.144 \\
83.395 \\
93.538\end{array}$ & $\begin{array}{r}4 \\
73 \\
77\end{array}$ & $\begin{array}{l}2.536 \\
1.142\end{array}$ & 2.220 & .075 \\
\hline & $\begin{array}{l}\text { Assume primary responsi- } \\
\text { bility for promotion of } \\
\text { religious life of students } \\
\text { at the institution via } \\
\text { delegation -- Expected }\end{array}$ & $\begin{array}{l}\text { Between Groups } \\
\text { Within Groups } \\
\text { Total. }\end{array}$ & $\begin{array}{r}8.548 \\
56.900 \\
65.449\end{array}$ & $\begin{array}{r}4 \\
73 \\
77\end{array}$ & $\begin{array}{r}2.137 \\
.779\end{array}$ & 2.742 & .035 \\
\hline \multirow{2}{*}{44.} & $\begin{array}{l}\text { Assume primary responsi- } \\
\text { bility for promotion of } \\
\text { religious life of faculty } \\
\text { and staff at the institution } \\
\text { via delegation -- Actual }\end{array}$ & $\begin{array}{l}\text { Between Groups } \\
\text { Within Groups } \\
\text { Total }\end{array}$ & $\begin{array}{r}9.079 \\
100.767 \\
109.846\end{array}$ & $\begin{array}{r}4 \\
73 \\
77\end{array}$ & $\begin{array}{l}2.270 \\
1.380\end{array}$ & 1.644 & .172 \\
\hline & $\begin{array}{l}\text { Assume primary responsi- } \\
\text { bility for promotion of } \\
\text { religious life of faculty } \\
\text { and staff at the institution } \\
\text { via delegation } \\
\text {-- Expected }\end{array}$ & $\begin{array}{l}\text { Between Groups } \\
\text { Within Groups } \\
\text { Total }\end{array}$ & $\begin{array}{r}4.799 \\
70.855 \\
75.654\end{array}$ & $\begin{array}{r}4 \\
73 \\
77\end{array}$ & $\begin{array}{r}1.200 \\
.971\end{array}$ & 1.236 & .303 \\
\hline \multirow{2}{*}{45.} & $\begin{array}{l}\text { Take ultimate responsi- } \\
\text { bility for midweek } \\
\text { chapels, vespers, weeks } \\
\text { of spiritual emphasis and } \\
\text { other religious programs } \\
\text { of the campus spiritual } \\
\text { nurture -- Actual }\end{array}$ & $\begin{array}{l}\text { Between Groups } \\
\text { Within Groups } \\
\text { Total }\end{array}$ & $\begin{array}{r}6.738 \\
130.916 \\
137.654\end{array}$ & $\begin{array}{r}4 \\
73 \\
77\end{array}$ & $\begin{array}{l}1.684 \\
1.793\end{array}$ & .939 & .446 \\
\hline & $\begin{array}{l}\text { Take ultimate responsi- } \\
\text { bility for midweek } \\
\text { chapels, vespers, weeks } \\
\text { of spiritual emphasis and } \\
\text { other religious programs } \\
\text { of the campus spiritual } \\
\text { nurture -- Expected }\end{array}$ & $\begin{array}{l}\text { Between Groups } \\
\text { Within Groups } \\
\text { Total }\end{array}$ & $\begin{array}{r}7.027 \\
109.345 \\
116.372\end{array}$ & $\begin{array}{r}4 \\
73 \\
77\end{array}$ & $\begin{array}{l}1.757 \\
1.498\end{array}$ & 1.173 & .330 \\
\hline
\end{tabular}


Table 53--Continued.

\begin{tabular}{|c|c|c|c|c|c|c|c|}
\hline No. & Variable & $\begin{array}{l}\text { Source of } \\
\text { Variance }\end{array}$ & $\begin{array}{c}\text { Sum of } \\
\text { Squares }\end{array}$ & $d f$ & $\begin{array}{l}\text { Mean } \\
\text { Square }\end{array}$ & $F$ & Sig. \\
\hline \multirow{2}{*}{46.} & $\begin{array}{l}\text { Manifest Christ-like } \\
\text { character in spiritual } \\
\text { leadership roles } \\
\text {-- Actual }\end{array}$ & $\begin{array}{l}\text { Between Groups } \\
\text { Within Groups } \\
\text { Total }\end{array}$ & $\begin{array}{r}4.219 \\
45.166 \\
49.385\end{array}$ & $\begin{array}{r}4 \\
73 \\
77\end{array}$ & $\begin{array}{r}1.055 \\
.619\end{array}$ & 1.705 & .158 \\
\hline & $\begin{array}{l}\text { Manifest Christ-like } \\
\text { character in spiritual } \\
\text { leadership roles } \\
\text {-- Expected }\end{array}$ & $\begin{array}{l}\text { Between Groups } \\
\text { Within Groups } \\
\text { Total }\end{array}$ & $\begin{array}{r}.762 \\
14.072 \\
14.833\end{array}$ & $\begin{array}{r}4 \\
73 \\
77\end{array}$ & $\begin{array}{l}.190 \\
.193\end{array}$ & .988 & .420 \\
\hline \multirow{2}{*}{47.} & $\begin{array}{l}\text { Focus on Christian } \\
\text { philosophy of institution } \\
\text {-- Actual }\end{array}$ & $\begin{array}{l}\text { Between Groups } \\
\text { Within Groups } \\
\text { Total }\end{array}$ & $\begin{array}{r}3.014 \\
42.935 \\
45.949\end{array}$ & $\begin{array}{r}4 \\
73 \\
77\end{array}$ & $\begin{array}{l}.753 \\
.588\end{array}$ & 1.281 & .285 \\
\hline & $\begin{array}{l}\text { Focus on Christian } \\
\text { philosophy of institution } \\
\text {-- Expected }\end{array}$ & $\begin{array}{l}\text { Between Groups } \\
\text { Within Groups } \\
\text { Total }\end{array}$ & $\begin{array}{r}.546 \\
7.416 \\
7.962\end{array}$ & $\begin{array}{r}4 \\
73 \\
77\end{array}$ & $\begin{array}{l}.136 \\
.102\end{array}$ & 1.343 & .262 \\
\hline
\end{tabular}


APPENDIX J

TABLE 54

COMPARATIVE LEVELS OF PERCEIVED

ACTUAL ROLE PERFORMANCES AND

EXPECTED ROLE RESPONSIBILITIES

OF PRESIDENTS IN RANK ORDER

OF HIGHEST TO LOWEST MEANS 
Table 54. Comparative levels of perceived actual role performances and expected role responsibilities of presidents in rank order of highest to lowest means

\begin{tabular}{|c|c|c|c|c|c|c|c|c|c|}
\hline \multicolumn{5}{|c|}{$\begin{array}{l}\text { Level 1: Very Strong Role Performance } \\
M=4.51-M=5.00\end{array}$} & \multicolumn{5}{|c|}{$\begin{array}{c}\text { EXPECTED } \\
\text { Level 1: Very High Role Expectation } \\
M=4.51-M=5.00\end{array}$} \\
\hline Role & $M$ & $S D$ & Rank & Item & Item & Rank & Role & $M$ & $S D$ \\
\hline Focus on Christian philosophy...... ${ }^{7}$ & 4.64 & .77 & 1.5 & 47 & -47 & 1 & Focus on Christian philosophy..... ${ }^{7}$ & 4.88 & .32 \\
\hline \multirow[t]{14}{*}{ Prepare board meeting agendas ${ }^{3}$} & 4.64 & .79 & 1.5 & 17. & 24 & 2 & Formulate .....educational philosophy ${ }^{3}$ & 4.87 & .37 \\
\hline & & & & & 38 & 3 & Represent institution to churches .... ${ }^{6}$ & 4.85 & .40 \\
\hline & & & & & 46 & 4 & Manifest Christ-like character ..... ${ }^{7}$ & 4.83 & .44 \\
\hline & & & & & -17 & 5 & Prepare board meeting agendas ${ }^{3}$ & 4.81 & .60 \\
\hline & & & & & 37 & 6 & Assist in fund-raising for institution & 4.77 & .64 \\
\hline & & & & & 20 & 7.5 & Responsible for strategic planning.... ${ }^{3}$ & 4.76 & .61 \\
\hline & & & & & 40 & 7.5 & Represent institution to public ${ }^{6}$ & 4.76 & .71 \\
\hline & & & & & 42 & 9 & Listen to constituency and alumni ${ }^{6}$ & 4.74 & .47 \\
\hline & & & & & 25 & 10 & Delegate .......to administrators....... ${ }^{3}$ & 4.73 & .53 \\
\hline & & & & & 39 & 11 & Present reports .... to community..... ${ }^{6}$ & 4.67 & .64 \\
\hline & & & & & 30 & 12 & Accountable ... for financial operation ${ }^{4}$ & 4.62 & .84 \\
\hline & & & & & 23 & 13 & Comply w/ laws to address grievances ${ }^{3}$ & 4.60 & .74 \\
\hline & & & & & 21 & 14 & Prepare \& review organizational chart ${ }^{3}$ & 4.53 & .75 \\
\hline & & & & & 41 & 15 & Associate closely w/ alumni groups ${ }^{6}$ & 4.51 & .62 \\
\hline
\end{tabular}


Table 54--Continued

\section{ACTUAL}

Level 2: Strong Role Performance $M=3.51-M=4.50$

Role

$M$

SD Rank Item

Represent institution to public ${ }^{6}$

4.49

.88

4.46

Manifest Christ-like character...... ${ }^{7}$

4.44

$.80 \quad 4 \quad 46$

Formulate.......educational philosophy ${ }^{3}$

4.40

$\begin{array}{lll}.92 & 5 & 24\end{array}$

Represent institution to churches......

4.29

.926

38

Responsible for strategic planning......

4.28

1.05

7

20

Present reports ... to community $\&$ alumni ${ }^{6}$

1.07

Comply w/ laws to address grievances..... ${ }^{3}$

4.26

Listen to constituency and alumni ${ }^{6}$

4.23

.93

Recommend......hiring, promotion,...... ${ }^{2}$

4.15

1.04

Delegate.....to administrators...... ${ }^{3}$

1.14

4.14

1.14

Assist in fund-raising for institution

1.15

Responsible for students' religious life ${ }^{7}$

1.10

Present to board the physical needs.....

4.06

1.24

Participate in faculty recruitment ${ }^{2}$

4.05

Associate closely with alumni groups ${ }^{6}$

Accountable.....for financial operation.... ${ }^{4}$

Prepare \& review organizational chart.... ${ }^{3}$

Preside at general faculty meetings ${ }^{3}$

Participate in.......budget preparation ${ }^{4}$

Foster research \& intellectual climate.... ${ }^{3}$

Responsible for faculty's religious life ${ }^{7}$

Dialogue frequently with students'

Review accrediting.... reports $w /$ faculty $^{2}$

Ascertain campus grounds are well kept ${ }^{5}$

Ensure.....school's budget is adhered to ${ }^{4}$

Guard faculty's academic freedom ${ }^{2}$

Ensure..... master plans are.......followed ${ }^{5}$

Carry out policies...... of faculty..... ${ }^{2}$ 


\section{EXPECTED}

\section{Level 2: High Role Expectation}

$$
M=3.51-M=4.50
$$

\section{Item Rank Role}

16 Responsible for students' religious life ${ }^{7}$

17 Ensure.....budget is adhered to ${ }^{4}$

Recommend......hiring, promotion,..... ${ }^{2}$

M

4.47

4.45

4.41

4.40

19 Participate in......budget preparation ${ }^{4}$

4.35

4.33

4.27

4.23

4.22

4.19

4.12

4.09

4.04

4.03

3.91

3.90

3.79

3.69

3.65

1.40

$29 \quad 33$

Recommend.......salary changes...... ${ }^{4}$

3.65

1.14

1534.5 Collaborate......in-service training......2

3.65

1.23

136

Participate in student recruitment ${ }^{1}$

$3.59 \quad 1.12$


Table 54--Continued

\begin{tabular}{|c|c|c|c|c|c|c|c|c|c|}
\hline \multicolumn{5}{|c|}{$\begin{array}{l}\text { Level 3: Moderate Role Performance } \\
M=2.51-M=3.50\end{array}$} & \multicolumn{5}{|c|}{ Level 3: $\begin{array}{l}\text { EXPECTED } \\
M=2.51-M=3.50\end{array}$} \\
\hline Role & $M$ & $S D$ & Rank & Item & Item & Rank & Role & $M$ & $S D$ \\
\hline Plan for new buildings and installations ${ }^{s}$ & 3.50 & 1.44 & 31 & 32 & .28 & 37 & Present....financial statement to board ${ }^{4}$ & 3.38 & 1.51 \\
\hline Collaborate...in-service training for faculty ${ }^{2}$ & 3.42 & 1.25 & 32 & 15 & 34 & 38 & Recommend...for equipment acquisition ${ }^{5}$ & 3.36 & 1.55 \\
\hline Responsible for religious programs ${ }^{7}$ & 3.35 & 1.34 & 33 & 45 & 8 & 39 & Serve as mentor of students ${ }^{1}$ & 3.33 & $1: 28$ \\
\hline Participate in student recruitment ${ }^{1}$ & 3.32 & 1.26 & 34 & 1 & 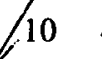 & 40 & Serve as academic leader of faculty ${ }^{2}$ & 3.31 & 1.26 \\
\hline Work.....with student government leaders ${ }^{1}$ & 3.29 & 1.23 & 35 & 2 & & 41 & Responsible for.....admission standards ${ }^{3}$ & 3.27 & 1.26 \\
\hline Recommend....salary changes for positions ${ }^{4}$ & 3.28 & 1.42 & 36 & 29 & & 42.5 & Responsible for......academic matters... ${ }^{2}$ & 3.21 & 1.38 \\
\hline Present financial statement to board ${ }^{4}$ & 3.12 & 1.47 & 37 & 28 & & 42.5 & Responsible for student discipline ${ }^{1}$ & 3.21 & 1.54 \\
\hline Plan for major repairs \& alterations....... ${ }^{5}$ & 3.05 & 1.39 & 38.5 & 33 & & 44 & Plan for major repairs \& alterations...... & 3.17 & 1.34 \\
\hline Recommend.....for equipment acquisition ${ }^{5}$ & 305 & 1.50 & 38.5 & $34^{\prime}$ & & 45 & Visit students in dorms...........................' & 3.05 & 1.25 \\
\hline Responsible for.... admission standards ${ }^{3}$ & 2.97 & 1.21 & 40.5 & $19^{\prime}$ & & 46 & Teach classes for contact with students' & 2.73 & 1.52 \\
\hline Serve as academic leader of faculty ${ }^{2}$ & 2.97 & 1.26 & 40.5 & & & & & & \\
\hline Responsible for academic matters ${ }^{2}$ & 2.96 & 1.31 & 42 & & & & & & \\
\hline Responsible for student discipline ${ }^{l}$ & 2.92 & 1.45 & 43 & & & & & & \\
\hline Serve as mentor of students ${ }^{1}$ & 2.91 & 1.27 & 44 & & & & & & \\
\hline Visit students in dorms during....worships ${ }^{1}$ & 2.55 & 1.24 & 45 & & & & & & \\
\hline \multicolumn{5}{|c|}{$\begin{array}{l}\text { Level 4: Weak Role Performance } \\
\qquad M=1.51-M=2.50\end{array}$} & \multicolumn{5}{|c|}{$\begin{array}{l}\text { Level 4: Low Role Expectation } \\
\qquad M=1.51-M=2.50\end{array}$} \\
\hline Role & $M$ & $S D$ & Rank & Item & Item $\mathrm{F}$ & Rank I & Role & $M$ & $S D$ \\
\hline Teach classes for contact with students' & 2.45 & 1.55 & 46 & 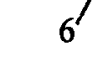 & 7 & 47 & Know every student personally ${ }^{1}$ & 2.41 & 1.32 \\
\hline Know every student personally' & 2.10 & 1.19 & 47 & & & & & & \\
\hline
\end{tabular}

Note: Superscript Code Explanation: 1 = Student-Related Functions; 2 = Employee-Related Functions; $3=$ General Administrative Functions; $4=$ FinanceRelated Functions; 5 = Physical Facilities-Related Functions; $6=$ Community- and Alumni-Related Functions; and $7=$ Spiritual Life-Related Functions. 


\section{APPENDIX K}

\section{TABLE 55}

COMPARATIVE LEVELS OF PERCEIVED

ACTUAL ROLE PERFORMANCES AND

EXPECTED ROLE RESPONSIBILITIES

BY CATEGORIES OF FUNCTIONS 
Table 55. Comparative levels of perceived actual role performances and expected role responsibilities of presidents by categories of functions

\begin{tabular}{|c|c|c|c|c|c|c|c|c|}
\hline \multirow{2}{*}{ Items } & \multicolumn{4}{|c|}{ Actual } & \multicolumn{4}{|c|}{ Expected } \\
\hline & $\begin{array}{c}\text { Level } 1 \\
\text { VSRP } \\
\end{array}$ & $\begin{array}{c}\text { Level } 2 \\
\text { SRP }\end{array}$ & $\begin{array}{c}\text { Level 3 } \\
\text { MRP } \\
\end{array}$ & $\begin{array}{c}\text { Level } 4 \\
\text { WRP }\end{array}$ & $\begin{array}{c}\text { Level 1 } \\
\text { VHRE }\end{array}$ & $\begin{array}{c}\text { Level } 2 \\
\text { HRE }\end{array}$ & $\begin{array}{c}\text { Level 3 } \\
\text { MRE }\end{array}$ & $\begin{array}{c}\text { Level 4 } \\
\text { LRE }\end{array}$ \\
\hline $\begin{array}{l}\text { I. Student-Related Functions } \\
\text { 1. Participate in student recruitment } \\
\text { 2. Work closely with student government leaders } \\
\text { 3. Dialogue frequently with students } \\
\text { 4. Visit students in dorms periodically during dorm worships } \\
\text { 5. Take ultimate responsibility for student discipline } \\
\text { 6. Teach some classes to maintain contact with students } \\
\text { 7. Know every student personally } \\
\text { 8. Serve as mentor of students }\end{array}$ & & $x$ & $\begin{array}{l}x \\
x \\
x \\
x \\
x\end{array}$ & $\begin{array}{l}x \\
x\end{array}$ & & $\begin{array}{l}x \\
x \\
x\end{array}$ & $\begin{array}{l}x \\
x \\
x \\
x\end{array}$ & $x$ \\
\hline $\begin{array}{l}\text { II. Employee-Related Functions } \\
\text { 9. Participate in faculty recruitment } \\
\text { 10. Serve as academic leader of faculty } \\
\text { 11. Guard faculty's academic freedom } \\
\text { 12. Be responsible for submitting proposed academic changes to faculty } \\
\text { 13. Work with faculty in reviewing accrediting agency's reports } \\
\text { 14. Recommend to board for hiring, promotion, demotion, and dismissal } \\
\text { of faculty and staff } \\
\text { 15. Collaborate for advanced in-service training for faculty and staff } \\
\text { 16. Carry out policies and regulations of faculty and staff }\end{array}$ & & $\begin{array}{l}x \\
\times \\
\times \\
x \\
x\end{array}$ & $\begin{array}{l}x \\
\times\end{array}$ & & & $\begin{array}{l}\times \\
\times \\
\times \\
\times \\
\times \\
\times\end{array}$ & $\begin{array}{l}x \\
x\end{array}$ & \\
\hline
\end{tabular}


Table 55-Continued.

\begin{tabular}{|c|c|c|c|c|c|c|c|c|}
\hline \multirow{2}{*}{ Items } & \multicolumn{4}{|c|}{ Actual } & \multicolumn{4}{|c|}{ Expected } \\
\hline & $\begin{array}{c}\text { Level } 1 \\
\text { VSRP }\end{array}$ & $\begin{array}{c}\text { Level } 2 \\
\text { SRP }\end{array}$ & $\begin{array}{c}\text { Level 3 } \\
\text { MRP }\end{array}$ & $\begin{array}{c}\text { Level } 4 \\
\text { WRP }\end{array}$ & $\begin{array}{l}\text { Level } 1 \\
\text { VHRE }\end{array}$ & $\begin{array}{c}\text { Level } 2 \\
\text { HRE }\end{array}$ & $\begin{array}{c}\text { Level } 3 \\
\text { MRE }\end{array}$ & $\begin{array}{c}\text { Level } 4 \\
\text { LRE }\end{array}$ \\
\hline $\begin{array}{l}\text { III. General Administrative Functions } \\
\text { 17. Prepare agenda for board of trustees' meetings } \\
\text { 18. Preside over general faculty meetings } \\
\text { 19. Take responsibility in structuring student admission standards } \\
\text { 20. Take responsibility in strategic planning for institution } \\
\text { 21. Take a leading role to prepare, revise, and follow organizational chart } \\
\text { 22. Foster research and intellectual climate for the institution } \\
\text { 23. Comply with legal mandates and laws in addressing employee grievances } \\
\text { 24. Help formulate and adhere to institution's educational philosophy } \\
\text { 25. Delegate responsibility, authority, and needed resources to appropriate } \\
\text { administrators and support their roles }\end{array}$ & $\times$ & $\begin{array}{l}x \\
x \\
x \\
x \\
x \\
x\end{array}$ & $x$ & & $\begin{array}{l}x \\
x \\
x \\
x \\
x\end{array}$ & $x$ & $x$ & \\
\hline $\begin{array}{l}\text { IV. Finance-Related Functions } \\
\text { 26. Participate in the preparation of annual budget of the institution } \\
\text { 27. Ensure that institution's budget, after approval by board, is adhered to } \\
\text { 28. Present institution's annual audited financial statements to board } \\
\text { 29. Recommend to board the salary changes for faculty/staff positions } \\
\text { 30. Accept accountability to board for financial operation of institution }\end{array}$ & & $\begin{array}{l}x \\
x \\
x\end{array}$ & $\begin{array}{l}x \\
x\end{array}$ & & $\times$ & $\begin{array}{l}x \\
x \\
x\end{array}$ & $x$ & \\
\hline $\begin{array}{l}\text { V. Physical Facilities-Related Functions } \\
\text { 31. Present to board the physical needs of institution } \\
\text { 32. Direct the preparation of plans for new buildings and installations } \\
\text { 33. Plan for major repairs and alterations of plant, buildings, and equipment } \\
\text { 34. Recommend to board for equipment acquisitions } \\
\text { 35. Ensure that physical plant master plans are current and followed closely } \\
\text { 36. Ascertain that campus grounds are well-kept through appropriate officer }\end{array}$ & & $\begin{array}{l}x \\
x\end{array}$ & $\begin{array}{l}x \\
x \\
x\end{array}$ & & . & $\begin{array}{l}x \\
x\end{array}$ & $\begin{array}{l}x \\
x\end{array}$ & \\
\hline
\end{tabular}




\begin{tabular}{|c|c|c|c|c|c|c|c|c|}
\hline \multirow[b]{2}{*}{ Items } & \multicolumn{4}{|c|}{ Actual } & \multicolumn{4}{|c|}{ Expected } \\
\hline & $\begin{array}{c}\text { Level } 1 \\
\text { VSRP }\end{array}$ & $\begin{array}{c}\text { Level } 2 \\
\text { SRP }\end{array}$ & $\begin{array}{c}\text { Level } 3 \\
\text { MRP }\end{array}$ & $\begin{array}{c}\text { Level 4 } \\
\text { WRP }\end{array}$ & $\begin{array}{l}\text { Level } 1 \\
\text { VHRE }\end{array}$ & $\begin{array}{c}\text { Level } 2 \\
\text { HRE }\end{array}$ & $\begin{array}{c}\text { Level } 3 \\
\text { MRE }\end{array}$ & $\begin{array}{c}\text { Level } 4 \\
\text { LRE }\end{array}$ \\
\hline $\begin{array}{l}\text { VI. Community- and Alumni-Related Functions } \\
\text { 37. Assist in raising funds for support of institution } \\
\text { 38. Represent institution to churches of the constituency } \\
\text { 39. Present reports about college/university to constituency and alumni } \\
\text { 40. Represent institution as its official spokesperson to public } \\
\text { 41. Associate closely with alumni groups } \\
\text { 42. Listen to constituency and alumni }\end{array}$ & & $\begin{array}{l}x \\
x \\
x \\
x \\
x \\
x\end{array}$ & & & $\begin{array}{l}x \\
x \\
x \\
x \\
x \\
x\end{array}$ & & & \\
\hline $\begin{array}{l}\text { VII. Spiritual Life-Related Functions } \\
\text { 43. Be responsible for promotion of religious life of students } \\
\text { 44. Be responsible for promotion of religious life of faculty and staff } \\
\text { 45. Be responsible for chapels, vespers, weeks of spiritual emphasis } \\
\text { and other religious programs } \\
\text { 46. Manifest Christ-like character in spiritual leadership roles } \\
\text { 47. Focus on Christian philosophy of institution }\end{array}$ & $x$ & $\begin{array}{l}x \\
x\end{array}$ & $x$ & & $\begin{array}{l}x \\
x\end{array}$ & $\begin{array}{l}x \\
x \\
x\end{array}$ & & \\
\hline $\begin{array}{l}\text { Totals } \\
\text { Percentages }\end{array}$ & $\begin{array}{c}2 \\
4.3\end{array}$ & $\begin{array}{c}28 \\
59.5\end{array}$ & $\begin{array}{c}15 \\
31.9\end{array}$ & $\begin{array}{c}2 \\
4.3\end{array}$ & $\begin{array}{c}15 \\
31.9\end{array}$ & $\begin{array}{c}21 \\
44.7\end{array}$ & $\begin{array}{c}10 \\
21.3\end{array}$ & $\begin{array}{c}1 \\
21.1\end{array}$ \\
\hline
\end{tabular}

Note: *Denotes perception level of actual role performances and expected role responsibilities of the presidents.

Explanation of level acronyms:

Actual: $\quad$ VSRP $=$ Very Strong Role Performance; $S R P=$ Strong Role Performance; $M R P=$ Moderate Role Performance; $W R P=W e e k$ Role Performance.

Expected: $\quad$ VHRE $=$ Very High Role Expectation; $H R E=$ High Role Expectation; MRE $=$ Moderate Role expectation; LRE $=$ Low Role Expectation. 


\section{APPENDIX L}

TABLE 56

RANKING OF FIVE STATUS GROUPS

FOR THEIR PERCEPTIONS AND EXPECTATIONS

OF SEVEN CATEGORIES OF

ACTUAL AND EXPECTED FUNCTIONS 
Table 56. Ranking of five status groups for their perceptions and expectations of seven categories of actual and expected functions, calculated in the order of the highest (1) to the lowest (5) means.

\begin{tabular}{|c|c|c|c|c|c|c|}
\hline \multirow[b]{2}{*}{$\begin{array}{l}\text { Category of } \\
\text { Function }\end{array}$} & \multirow[b]{2}{*}{$\begin{array}{c}\text { Type of } \\
\text { Role }\end{array}$} & \multicolumn{5}{|c|}{ Status Groups } \\
\hline & & $\begin{array}{l}\text { Board } \\
\text { Chair }\end{array}$ & President & $\begin{array}{l}\text { Academic } \\
\text { Vice } \\
\text { President }\end{array}$ & $\begin{array}{l}\text { Student } \\
\text { Service } \\
\text { Vice } \\
\text { President }\end{array}$ & $\begin{array}{l}\text { Finance } \\
\text { Vice } \\
\text { President }\end{array}$ \\
\hline \multirow{2}{*}{$\begin{array}{l}\text { I. Student-Related } \\
\text { Functions }\end{array}$} & Actual & 2 & 1 & 5 & 4 & 3 \\
\hline & Expected & 1 & 3 & 5 & 4 & 2 \\
\hline \multirow{2}{*}{$\begin{array}{l}\text { II. Employee-Related } \\
\text { Functions }\end{array}$} & Actual & 1 & 2 & 5 & 4 & 3 \\
\hline & Expected & 1 & 4 & 5 & 2 & 3 \\
\hline \multirow{2}{*}{$\begin{array}{l}\text { III. General } \\
\text { Administrative } \\
\text { Functions }\end{array}$} & Actúal & 2 & 1 & 5 & 3 & 4 \\
\hline & Expected & 1 & 4 & 2 & 3 & 5 \\
\hline \multirow{2}{*}{$\begin{array}{l}\text { IV. Finance-Related } \\
\text { Functions }\end{array}$} & Actual & 4 & 1 & 3 & 2 & 5 \\
\hline & Expected & 2 & 4 & 1 & 3 & 5 \\
\hline \multirow{2}{*}{$\begin{array}{l}\text { V. Physical Facilities- } \\
\text { Related Functions }\end{array}$} & Actual & 1 & 2 & 3 & 4 & 5 \\
\hline & Expected & 3 & 1 & 2 & 4 & 5 \\
\hline \multirow{2}{*}{$\begin{array}{l}\text { VI. Community- and } \\
\text { Alumni-Related } \\
\text { Functions }\end{array}$} & Actual & 3 & 1 & 4 & 2 & 5 \\
\hline & Expected & 3 & 2 & 4 & 1 & 5 \\
\hline \multirow{2}{*}{$\begin{array}{l}\text { VII. Spiritual Life- } \\
\text { Related } \\
\text { Functions }\end{array}$} & Actual & 2 & 1 & 5 & 4 & 3 \\
\hline & Expected & 1 & 2 & 3 & 5 & 4 \\
\hline \multirow{2}{*}{$\begin{array}{l}\text { All Categories of } \\
\text { Functions (I-VII) }\end{array}$} & Actual & 2 & 1 & 5 & 3 & 4 \\
\hline & Expected & 1 & 2 & 3 & 4 & 5 \\
\hline
\end{tabular}

Note: 1=First; $2=$ Second; $3=$ Third; $4=$ Fourth; and 5=Fifth. The numbers indicate the response ranks of the five groups for their perceptions and expectations of each of the seven categories of actual and expected functions. The ranks were determined from the means presented in Table 15. 
APPENDIX M

TABLE 57

RANKING OF SEVEN CATEGORIES OF

ACTUAL AND EXPECTED FUNCTIONS

AS PERCEIVED BY STATUS GROUPS 
Table 57. Ranking of seven categories of actual and expected functions as perceived by the status groups, rank ordered by means, calculated in the order of the highest (1) to the lowest (7) group means

\begin{tabular}{|c|c|c|c|c|c|c|c|c|c|c|c|c|}
\hline \multirow{3}{*}{$\begin{array}{l}\text { Category } \\
\text { of } \\
\text { Functions }\end{array}$} & \multicolumn{10}{|c|}{ Status Groups } & \multirow{2}{*}{\multicolumn{2}{|c|}{$\begin{array}{c}\text { Average } \\
\text { of All } \\
\text { Five } \\
\text { Status } \\
\text { Groups } \\
\text { Combined }\end{array}$}} \\
\hline & \multicolumn{2}{|c|}{$\begin{array}{l}\text { Board } \\
\text { Chair }\end{array}$} & \multicolumn{2}{|c|}{ President } & \multicolumn{2}{|c|}{$\begin{array}{l}\text { Academic } \\
\text { Vice } \\
\text { President }\end{array}$} & \multicolumn{2}{|c|}{$\begin{array}{l}\text { Student } \\
\text { Service } \\
\text { Vice } \\
\text { President }\end{array}$} & \multicolumn{2}{|c|}{$\begin{array}{l}\text { Finance } \\
\text { Vice } \\
\text { Presiden }\end{array}$} & & \\
\hline & A & E & A & $\mathrm{E}$ & A & $\mathrm{E}$ & A & $\mathrm{E}$ & A & $\mathrm{E}$ & A & $\mathrm{E}$ \\
\hline $\begin{array}{l}\text { I. Student-Related } \\
\text { Functions }\end{array}$ & 7 & 7 & 7 & 7 & 7 & 7 & 7 & 7 & 6 & 6 & 7 & 7 \\
\hline $\begin{array}{l}\text { II. Employee-Related } \\
\text { Functions }\end{array}$ & 5 & 5 & 5 & 6 & 6 & 6 & 5 & 5 & 4 & 4 & 5 & 5 \\
\hline $\begin{array}{l}\text { III. General Adminis- } \\
\text { trative Functions }\end{array}$ & 1 & 3 & 3 & 3 & 2 & 2 & 2 & 2 & 2 & 3 & 3 & 3 \\
\hline $\begin{array}{l}\text { IV. Finance-Related } \\
\text { Functions }\end{array}$ & 6 & 4 & 4 & 5 & 4 & 4 & 4 & 4 & 5 & 5 & & 4 \\
\hline $\begin{array}{l}\text { V. Physical Facilities- } \\
\text { Related Functions }\end{array}$ & 4 & 6 & 6 & 4 & 5 & 5 & 6 & 6 & 7 & 7 & 6 & 6 \\
\hline $\begin{array}{l}\text { VI. Community- and } \\
\text { Alumni-Related } \\
\text { Functions }\end{array}$ & 2 & 2 & 1 & 1 & 1 & 1 & 1 & 1 & 3 & 1 & 1 & 1 \\
\hline $\begin{array}{l}\text { VII. Spiritual Life- } \\
\text { Related Functions }\end{array}$ & 3 & 1 & 2 & 2 & 3 & 3 & 3 & 3 & 1 & 2 & 2 & 2 \\
\hline
\end{tabular}

Note: A= Actual Roles; $\mathrm{E}=$ Expected Roles. $1=$ First; $2=$ Second; $3=$ Third; $4=$ Fourth; $5=$ Fifth; $6=$ Sixth; and $7=$ Seventh. The numbers indicate the ranks among the seven categories of functions calculated from the means as presented in Table 15. 


\section{TABLE N}

TABLE 58

RANKING OF SIXTEEN ITEMS

HAVING NO SIGNIFICANT DIFFERENCES

BETWEEN ACTUAL AND EXPECTED ROLES 
Table 58. Ranking of sixteen items having no significant statistical differences between actual and expected roles, arranged in the order of the highest no-significance level to the lowest no-significance level $(p>0.001)$

\begin{tabular}{|c|c|l|c|}
\hline Rank & Item \# & \multicolumn{1}{|c|}{ Item } & t-value \\
\hline 1 & 18 & Preside over general faculty meetings & -0.418 \\
\hline 2 & 33 & Plan for major repairs or alterations of plants, buildings and equipment & -1.136 \\
\hline 3 & 6 & Teach some classes in order to maintain contact with the students & -1.949 \\
\hline 4 & 5 & Take ultimate responsibility for student discipline & -1.997 \\
\hline 5 & 15 & Collaborate for advanced in-service training for faculty & -2.081 \\
\hline 6 & 9 & Participate in faculty recruitment & -2.188 \\
\hline 7 & 17 & Prepare agenda for board of trustees' meetings & -2.493 \\
\hline 8 & 1 & Participate in student recruitment & -2.506 \\
\hline 9 & 16 & Carry out policies and regulations of faculty and staff & -2.511 \\
\hline 10 & 32 & Direct the preparation of plans for new buildings and installations & -2.727 \\
\hline 11 & 47 & Focus on Christian philosophy of institution & -2.772 \\
\hline 12 & 12 & Have responsibility in submitting proposed academic changes to faculty & -2.834 \\
\hline 13 & 31 & Present to the board the physical needs of the institution & -2.857 \\
\hline 14 & 7 & Know every student personally & -2.966 \\
\hline 15 & 36 & Ascertain that campus grounds are well-kept through appropriate officer & -3.013 \\
\hline 16 & 10 & Serve as academic leader of the faculty & -3.017 \\
\hline
\end{tabular}

Note: The rankings were based on the $t$-test results presented in Table 29. 
TABLE O

TABLE 59

RANKING OF THIRTY-ONE ITEMS

HAVING SIGNIFICANT STATISTICAL

DIFFERENCES BETWEEN

ACTUAL AND EXPECTED ROLES 
Table 59. Ranking of thirty-one items having significant statistical differences between actual and expected roles, in the order of highest to lowest significance level at $p \leq 0.001$

\begin{tabular}{|c|c|c|c|}
\hline Rank & Item & Item & $t$-value \\
\hline 1 & 27 & Ensure that institution's budget, after approved by the board, is adhered to & -6.007 \\
\hline 2 & 37 & Assist in raising funds for the support of the institution & -5.712 \\
\hline 3 & 30 & Accept accountability to the board for financial operation of the institution & -5.500 \\
\hline 4 & 44 & Assume primary responsibility for promotion of religious life of faculty & -5.461 \\
\hline 5 & 45 & Take ultimate responsibility for chapels, vespers, spiritual emphasis week, etc. & -5.256 \\
\hline 6 & 38 & Represent the institution to the churches of the constituency & -4.855 \\
\hline 7 & 11 & Guard academic freedom of the faculty & -4.608 \\
\hline 8 & 25 & Delegate responsibility, authority, and needed resources to administrators & -4.551 \\
\hline 9 & 21 & Take a leading role in preparing and revising the organizational chart & -4.529 \\
\hline 10 & 13 & Work with the faculty in reviewing the accrediting agency's reports & -4.460 \\
\hline 11 & 42 & Listen to the constituency and alumni & -4.458 \\
\hline 12 & 41 & Associate closely with the alumni groups & -4.357 \\
\hline 13 & 24 & Help formulate and adhere to the institution's educational philosophy & -4.319 \\
\hline 14 & 29 & Recommend to the board the salary changes for positions & -4.292 \\
\hline 15 & 23 & Comply with the legal mandates and laws in addressing the grievances & -4.260 \\
\hline 16 & 39 & Present reports about the institution to the community and alumni & -4.196 \\
\hline 17 & 22 & Foster research and intellectual climate for the institution & -4.015 \\
\hline 18 & 46 & Manifest Christ-like character in spiritual leadership roles & -3.913 \\
\hline 19 & 8 & Serve as mentor of students & -3.890 \\
\hline 20 & 20 & Take responsibility in strategic planning for the institution & -3.871 \\
\hline 21 & 26 & Participate in the preparation of the annual operating budget of the institution & -3.803 \\
\hline 22 & 4 & Visit students periodically in the dorms during the dorm worships & -3.773 \\
\hline 23 & 19 & Take responsibility in structuring student admission standards & -3.697 \\
\hline 24 & 34 & Recommend to the board for equipment acquisition & -3.653 \\
\hline 25.5 & 43 & Assume primary responsibility for promotion of religious life of students & -3.613 \\
\hline 25.5 & 40 & Represent institution as its official spokesperson to public & -3.613 \\
\hline 27 & 2 & Work closely with student government leaders & -3.593 \\
\hline 28 & 35 & Ensure that the campus grounds are well-kept through appropriate officer & -3.588 \\
\hline 29 & 3 & Dialogue frequently with the students & -3.484 \\
\hline 30 & 14 & Recommend to board for hiring, promotion, demotion, \&dismissal of faculty & -3.365 \\
\hline 31 & 28 & Present the institution's annual audited financial statements to the board. & -3.326 \\
\hline
\end{tabular}

Note: The rankings were based on the $t$-test results presented in Table 29 . 
BIBLIOGRAPHY 


\section{BIBLIOGRAPHY}

Acker, Duane. Can State Universities Be Managed? A Primer for Presidents and Management Teams. Westport, CT: Praeger Publishers, 2006.

Adams, Jacob E., and Michael W. Kirst. "New Demands and Concepts for Educational Accountability: Striving for Results in an Era of Excellence." In Handbook of Research on Educational Administration, edited by Joseph Murphy and Karen Seashore Louis, 465. $2^{\text {nd }}$ ed. San Francisco, CA: Jossey-Bass, 1999.

Akker, John. "Protecting Academic Freedom Worldwide." ACADEME: Bulletin of the American Association of University Professors 88, no. 3 (2002): 44-45.

Aleshire, Daniel, Cynthia Campbell, and Kevin Mannoia. "The President's Vocation and Leadership." In A Handbook for Seminary Presidents, edited by G. Douglass Lewis and Lovett H. Weems, Jr., 7-8. Grand Rapids, MI: William B. Eerdmans Publishing, 2006.

Altbach, Philip G., and Toru Umazkoshi, eds. Asian Universities: Historical Perspectives and Contemporary Challenges. Baltimore, MD: Johns Hopkins University Press, 2004.

Amaral, Alberto, Vincent Lynn Meek, and Ingvild Marheim Larsen, eds. The Higher Education Managerial Revolution? Higher Education Dynamics Series 2003. www.springer.com/education/journal/11162 (accessed March 27, 2008).

American University. "Cornelius M. Kerwin: Biography." www1.american.edu/president/bio/index.html (accessed May 8, 2009).

Amrein-Beardsley, Audrey. "Recruiting Expert Teachers Into Hard-to-Staff Schools." The Education Digest 73, no. 4 (2007): 41.

Andreasen, Niels-Erik. "President's Annual Report-2008." Focus: The Andrews University Magazine 44, no. 4 (2008): 48.

."Servant to the World Church." Focus: The Andrews University Magazine 44, no. 2 (2008): 15.

Andrews University. "General Conference Statement on Academic Freedom in Seventhday Adventist Institutions of Higher Education." Andrews University Working Policy, Section 2, 2:801. www.andrews.edu/faculty/documents/index.html (accessed May 20, 2008). 
. Andrews University Working Policy. Duties of the President.

www.andrews.edu/faculty/documents/index.html (accessed May 20, 2008).

Association of Physical Plant Administrators of Universities and Colleges. Facilities Manager 21, no. 3 (2005): 24-43.

Atlantic Union College. Faculty Working Policy and Handbook. South Lancaster, MA: Office of the President, 1998.

Avondale College. Unpublished manuscript. Cooranbong, N.S.W. Australia: Office of the President, 1988.

Bagin, Don, and Donald R. Gallagher. "The School and Community Relations." Needham Heights, MA: Allyn \& Bacon, 2001, 117. Quoted in Garret W. Nudd, "Practical Public Relations: Making Your School Stand Out Despite Limited Financial Resources." The Journal of Adventist Education 64, no. 1 (2001): 9.

Basinger, Rebekah Burch, C. Samuel Calian, and Robert F. Leavitt. "The President's Role in Institutional Advancement." In A Handbook for Seminary Presidents, edited by G. Douglass Lewis and Lovett H. Weems, Jr., 127-137. Grand Rapids, MI: William B. Eerdmans Publishing, 2006.

Bauer, Ronald C. Cares in College Administration. London, England: Teachers College, Columbia University, 1959.

Beach, Bert B. "Can a University Be Christian? A Look at Academic Freedom on the Adventist Campus." Adventist Review: The General Paper of the Seventh-day Adventist Church 166, no. 9 (1989): 19-22.

Biddle, Bruce J. "Recent Development in Role Theory." Annual Review of Sociology 12 (1986): 67-92.

. Role Theory: Expectations, Identities, and Behaviors. New York, NY: Academic Press, 1979.

Biddle, Bruce J., and Edwin J. Thomas, eds. Role Theory: Concepts and Research. New York, NY: JohnWiley, 1966.

Birkenstock, David. "Leadership: The Key Dimension in Adventist Tertiary Educational Administration." In Christ in the Classroom: Adventist Approaches to the Integration of Faith and Learning, edited by Humbarto M. Rasi, 12:37-46. Silver Spring, MD: Institute for Christian Teaching, 1994. 
Blackwell, Thomas Edward. College and University Administration. New York, NY: Center for Applied Research in Education, 1966.

Bolivia Adventist University (SALT). "Structure and Administrative Organization." Vinto Cochabamba, Bolivia: Office of the President, 1998.

Bolton, Earl C., and Fredric H. Genck. "Universities and Management." The Journal of Higher Education 13 (1971): 281.

Bouchard, Charles E., Susan Thistlethwaite, and Timothy Weber. "The President's Role as Academic Leader." In $A$ Handbook for Seminary Presidents, edited by G. Douglass Lewis and Lovett H. Weems, Jr., 73-88. Grand Rapids, MI: William B. Eerdmans Publishing, 2006.

Boughman, Larry W. "Guidelines for Campus Beautification with Special Reference to Seventh-day Adventist Academies and Colleges in North America." Ph.D. dissertation, Andrews University, 1991.

Brazil Adventist University (SALT). "Structure and Administrative Organization." Engenheiro Coelho, SP, Brazil: Office of the President, 1998.

Brown, Walton J. A Handbook for Seventh-day Adventist School Administrators. Takoma Park, MD: Department of Education, General Conference of Seventh-day Adventists, 1980.

Budig, Gene A. “A Perfect Storm.” Phi Delta Kappan 88, no. 2 (2006): 114.

Caldwell, Garrett. "Atlanta 2010: Seventh-day Adventist Church: Proclaiming God's Grace." Lake Union Herald 102, no. 1 (2010): 30.

Calvin College. "Job Description of President." Grand Rapids, MI: Office of the President, 1998.

Campbell, Roald F., and Russell T. Gregg. Administrative Behavior in Education. New York, NY: Harper, 1957.

Campbell, Ronald F., John E. Corbally, and John B. Ramsayer. Introduction to Administration. Boston, MA: Allyn and Bacon, 1953.

Canadian University College. Faculty Handbook. Alberta, Canada: Office of the President, 1998. 
Caribbean Union College. Unpublished manuscript. Trinidad, West Indies: Office of the President, 1998.

Castaldi, Basil. Educational Facilities: Planning, Modernization, and Management. Boston, MA: Allyn and Bacon, 1994. 316-336.

Chase, Francis S. "How to Meet Teachers' Expectations of Leadership." Administrative Notebook, April 1953.

Chile Adventist University (SALT). "Structure and Administrative Organization." Chillan, Chile: Office of the President, 1998.

Clark College. "College President's Role in Equipment Acquisition." www.clarkcollegefoundation.org/newsletter.php?id=15 (accessed July 7, 2009.

Clark, Lita Allen. "Role Expectations for the Chief Executive Officer of a University System." Ph.D. dissertation, University of Alabama, 1977.

Cohen, Arthur M. The Shaping of American Higher Education: Emergence and Growth of the Contemporary System. San Francisco, CA: Jossey-Bass, 1998.

Cohen, Michael D., and James G. March. Leadership and Ambiguity: The American College President. Boston, MA: Harvard Business School Press, 1974.

Colombia Adventist University. Unpublished document. Colombia, MD: Office of the Rector, 1998.

Cooley, Robert, Christa Klein, and Louis Weeks. "The President's Role in Governance." In $A$ Handbook for Seminary Presidents, edited by G. Douglass Lewis and Lovett H. Weems, Jr., 36-57. Grand Rapids, MI: William B. Eerdmans Publishing, 2006.

Cornette, James P. “The President and His Faculty." Peabody Journal of Education 26, no. 5 (1949): 264-267.

Corson, John J. Governance of Colleges and Universities. New York, NY: McGraw-Hill, 1960.

Covington, Douglas. "Forging a Unified Vision Among Diverse Constituencies." In The Advancement President and the Academy: Profiles in Institutional Leadership, edited by Mary Kay Murphy, 99. Phoenix, AZ: Oryx Press, 1997.

Cunningham, William G., and Paula A. Cordeiro. Educational Leadership: A Bridge to Improved Practice. Boston, MA: Pearson Education, 2009. 
Cushing, Vincent, Donn Morgan, and Albert Aymer. "The President's Role in External Authorities." In $A$ Handbook for Seminary Presidents, edited by G. Douglass Lewis and Lovett H. Weems, Jr., 157-162. Grand Rapids, MI: William B. Eerdmans Publishing, 2006.

Davis, Stephen H. "Bridging the Gap Between Research and Practice: What's Good, What's Bad, and How Can One Be Sure?" Phi Delta Kappan 88, no. 8 (2007): 569-578.

Department of Education, General Conference of Seventh-day Adventists. "World Education Statistics December 31, 2004." The Journal of Adventist Education 67, no. 5 (2005): 2.

Department of Education, General Conference of Seventh-day Adventists. "Seventh-day Adventists and Scholarship." The Journal of Adventist Education 67, no. 5 (2005): 16-17.

Department of Education, General Conference of Seventh-day Adventists.

"Characteristics of a Successful Seventh-day Adventist College or University." Silver Spring, MD: General Conference, 1997.

Department of Education, General Conference of Seventh-day Adventists. "Suggested Topics for Research in Adventist Education." Compiled by Humbarto M. Rasi. Silver Spring, MD, March 1995.

Dodds, Harold W. The Academic President: Educator or Caretaker? With the collaboration of Felix C. Robb and E. Robb Taylor. New York, NY: McGrawHill, 1962.

Donovan, H. L., and R. W. Wild. "The President and the Public." Peabody Journal of Education 26, no. 5 (2000): 258-263.

Dove, Kent E., Jeffry A. Lindauer, and Carolyn P. Madvig. Conducting a Successful Annual Giving Program. San Francisco, CA: Jossey-Bass, 2001.

Dowdall, Jean A. Searching for Higher Education Leadership: Advice for Candidates and Search Committees. Westport, CT: Praegars Publishers, 2007.

Drozdowski, Mark J. "No Strings Attached." The Chronicle of Higher Education 54, no. 21 (2008): C 3.

Duderstadt, James J. The Vew from the Helm: Leading the American University During an Era of Change. Ann Arbor, MI: The University of Michigan Press, 2007. 
Edelbach, Gene. "Adventist Education: Mapping Your Future." Adventist Review, online ed. www.adventistreview.org/9944/story2.html (accessed December 28, 2009).

Ewing, Ward, Thomas Graves, and Robert Landrebe. "The President's Role in Managing Facilities." In A Handbook for Seminary Presidents, edited by G. Douglass Lewis and Lovett H. Weems, Jr., 106-124. Grand Rapids, MI: William B. Eerdmans Publishing, 2006.

Fisher, James L., and James V. Koch. Presidential Leadership: Making a Difference. Phoenix, AZ: American Council on Education and Oryx Press, 1996.

Fisher, James L., Martha W. Tack, and Karen J. Wheeler. The Effective College President. New York, NY: American Council on Education and Macmillan, 1988.

Fite, Harley. "The College President and the Church." Peabody Journal of Education 26, no. 5 (1949): 268-271.

Flawn, Peter T. A Primer for University Presidents: Managing the Modern University. Austin, TX: University of Texas Press, 1990.

Follett, Mary Parker. Creative Experience. New York, NY: Longmans, 1924.

Fowler, John M. "Philosophy and Christian Education: A Road to Despair or a Highway to Understanding?" The Journal of Adventist Education 69, no. 2 (2007): 4-9.

Furst, Lyndon G., and Charles J. Russo. The Legal Aspects of Nonpublic Schools: A Casebook. Berrien Springs, MI: Andrews University, 1993.

Gallimore, Jay. "Evolution in Education?" Michigan Memo: Official Newsletter for the Michigan Conference of Seventh-Day Adventists 21, no. 5 (2009): 2.

General Conference of Seventh-day Adventists. 144 th Annual Statistical Report 2006. Silver Spring, MD: Office of Archives and Statistics, 2006.

General Conference of Seventh-day Adventists. Working Policy, 2007-2008. Hagerstown, MD: Review and Herald, 2005, 241, 261-262.

Geraty, Lawrence T. "The Essential Characteristics of SDA Higher Education." The Journal of Adventist Education 56, no. 4 (1994): 5-10.

Getzels, Jacob W., and E. G. Guba. "The Structure of Roles and Role Conflict in a Teaching Situation." Journal of Educational Psychology 24 (1995): 30-40. 
Getzels, Jacob, James Lipham, and Ronald F. Campbell. Educational Administration as a Social Process. New York, NY: Harper and Row, 1968.

Gonzalez, Andrew. "The Philippines: Past, Present, and Future Dimensions of Higher Education." In Asian Universities: Historical Perspectives and Contemporary Challenges, edited by Philip G. Altbach and Toru Umazkoshi. Baltimore, MD: Johns Hopkins University Press, 2004.

Goonen, Norma M., and Rachel S. Blechman. Higher Education Administration: A Guide to Legal, Ethical, and Practical Issues. Westport, CT: Greenwood Press, 1999.

Gray, Meredith Jones. "Battle Creek College 1874-1901." In Christ in the Classroom: Adventist Approaches to the Integration of Faith and Learning, edited by Humberto M. Rasi, 24:138. Silver Spring, MD: Institute for Christian Teaching, 1999.

Green, Samuel B., and Neil J. Salkind. Using SPSS for Windows and Macintosh: Analyzing and Understanding Data. Upper Saddle River, NJ: Prentice Hall, 2007.

Greenleaf, Floyd. "Timeline for Seventh-day Adventist Education." The Journal of Adventist Education 67, no. 5 (2005): 10.

Guilford, J. P. Psychometric Methods. New York, NY: McGraw-Hill, 1954.

Gunsalus, C. K. The College Administrator's Survival Guide. Cambridge, MA: Harvard University Press, 2006.

Guskin, Alan E., and Mary B. Marcy. "Pressures for Fundamental Reform: Creating a Viable Academic Future." In Field Guide to Academic Leadership, edited by Robert M. Diamond, 3-14. San Francisco, CA: Jossey-Bass, 2002.

Haitian Adventist University. Unpublished manuscript. Port-au-Prince, Haiti: Office of the President, 1998.

Halpin, Andrew W. Administrative Theory in Education. London, England: CollierMacmillan, 1970.

Hansen, Adolf, Jean Stairs, and Kent M. Weeks. "The President's Role in Administration and Personnel Management." In $A$ Handbook for Seminary Presidents, edited by G. Douglass Lewis and Lovett H. Weems, Jr., 19-31. Grand Rapids, MI: William B. Eerdmans Publishing, 2006. 
Hansen, Arthur G. "President and Physical Plant Administrator Face Common Goal of Providing and Efficient Educational Environment." Association of Physical Plant Administrators of Universities and Colleges 5, no. 1 (1975): 1-7.

Hanson, Mark E. Educational Administration and Organizational Behavior. $5^{\text {th }}$ ed. Boston, MA: Allyn and Bacon, 2003.

Harvard University Allston Campus. "University President's Role in Master Planning." Retrieved on July 7, 2009 from www.allston.harvard.edu/imp/imp.html (accessed July 7, 2009.

Harvard University. "Summary of President's Duties." Cambridge, MA: Office of the President, Harvard College, 1998.

Harvey, William R. Inaugural speech by Dr. Harvey installing Dr. JoAnn W. Haysbert as new president of Langston University. www.hamptonu.edu/administration/president/speeches/haysbert_inauguration/html (accessed January 25, 2008).

Hausmann, Sister Daniel M. "The Role of the President in American Four-Year Liberal Arts Colleges Conducted by the Benedictine Sisters." Ph.D. dissertation, The Catholic University of America, 1963.

Helderberg College. Unpublished manuscript. Cape, South Africa: Office of the Rector, 1998.

Hesburgh, Theodore M. "Where Are College Presidents' Voices on Important Public Issues?" The Chronicle of Higher Education, February 2, 2001.

Hoy, Wayne K., and Cecil G. Miskel. Educational Administration: Theory, Research and Practice. $6^{\text {th }}$ ed. New York, NY: McGraw-Hill, 2001.

Hunt, Larry W. "A Study of the Perceptions of Presidents and Academic Deans Regarding Role Expectations and Decision-Making Styles of Academic Deans in Texas Community Colleges." Ph.D. dissertation, North Texas State University, 1977.

Hutchins, Henry Thomas, Jr. "Role Expectations of Selected College and University Presidents." Ph.D. dissertation, University of Oklahoma, 1962.

Ingraham, Mark H, and Francis P. King. The Mirror of Brass: The Compensation and Working Condition of College and University Administrators. Madison, WI: University of Wisconsin Press, 1968. 
Isaac, S., and W. Michael. Handbook in Research and Evaluation. San Diego, CA: EDITS, 1979.

Jayaram, N. "Higher Education in India: Massification and Change." In Asian Universities: Historical Perspectives and Contemporary Challenges, edited by Philip G. Altbach and Toru Umazkoshi. Baltimore, MD: Johns Hopkins University Press, 2004.

Jenkins, John I. "The Role of a Catholic University in the $21^{\text {st }}$ Century." www.controller.nd.edu/files/reports/2006-president.pdf (accessed January 22, 2008).

Johnston, Gladys Styles, and Carol Camp Yeakey, eds. Research and Thought in Administrative Theory: Developments in the Field of Educational Administration. Lanham, MD: University Press of America, 1986.

Kahn, Robert L. Organizational Stress: Studies in Role Conflict and Ambiguity. New York, NY: John Wiley, 1961.

Kalangi, Robert Albert. “A Comparative Study of the Perception of the Principal's Role as Held by Teachers and Principals in the Seventh-day Adventist Academies in the United States." Ph.D. dissertation, University of Nebraska, 1975.

Kamwendo, Elden G. "The Role of Administrative and Support Units of an Adventist University in Fostering Integration of Faith and Learning." In Christ in the Classroom: Adventist Approaches to the Integration of Faith and Learning, edited by Humberto M. Rasi, 28:2-19, 142. Silver Spring, MD: Institute for Christian Teaching, 2001.

Katz, Daniel, and Robert L. Kahn. The Social Psychology of Organizations. New York, NY: John Wiley, 1966.

Keating, Patrick Joseph. "Models for Campus Master Planning and Facility Development: A Comprehensive Case Study Analysis of Four Private Research Universities." Ph.D. dissertation, Michigan State University, 1988.

Keohane, Nannerl O. Higher Ground: Ethics and Leadership in the Modern University. Durham, NC: Duke University Press, 2006.

Kerr, C. The Uses of the University. Cambridge, MA: Harvard University Press, 1964.

Kim, Chong Wha. "A Study of Role Expectation of Presidents in Seventh-day Adventist Colleges." Ph.D. dissertation, University of Nebraska, 1975. 
King, Greg A. "Should Adventist Colleges Require Religion Classes?" The Journal of Adventist Education 68, no. 3 (2006): 22.

Klimes, Rudolph E., ed. Studies in Seventh-day Adventist Higher Educational Administration. Berrien Springs, MI: Andrews University, 1979.

, ed. Presidents and Boards in Seventh-day Adventist Colleges. Berrien Springs, MI: Andrews University, 1979.

Knight, George R. "Why Have Adventist Education?" The Journal of Adventist Education 67, no. 5 (2005): 6-9.

Koplik, Stanley Z. "A Study of Perception of Presidential Effectiveness at a Selected Group of Major Public and Private Universities and Colleges in the United States." Ph.D. dissertation, University of Kansas, 1985.

La Sierra University. Faculty Handbook. Riverside, CA: University Press, 1999.

Lall, Bernard Mohan. "Role Expectations of the School Superintendent as Perceived by Superintendents, Principals, Teachers, and Board Members in the Province of Saskatchewan." Ph.D. dissertation, University of Oregon, 1967.

Lall, Bernard Mohan, and Geeta Rani Lall. New Dynamic Leadership: How to Become an Effective Leader in a Contemporary Society. Berrien Springs, MI: Geetanjali Publishers, 1994.

Lashley, Sylvan. "Should Adventist Colleges and Universities Differ From One Another?" The Journal of Adventist Education 65, no. 2 (2002/2003): 7-9.

Law, Glen Charles. The Urgency of New Leadership in Higher Education. Stamford, CT: Press-Tige Publishing, 1962.

Lawrence, Francis L. Leadership in Higher Education: Views from the Presidency. New Brunswick, NJ: Transaction Publishers, 2006.

Lee, Sungho H. "Korean Higher Education: History and Future Challenges." In Asian Universities: Historical Perspectives and Contemporary Challenges, edited by Philip G. Altbach and Toru Umazkoshi. Baltimore, MD: Johns Hopkins University Press, 2004.

Leithwood, Kenneth, and Lorna Earl. "Educational Accountability Effects: An International Perspective." Peabody Journal of Education 75, no. 4 (2000): 6-7. 
Lenski, Gerhard. Human Societies: A Macrolevel Introduction to Sociology. New York, NY: McGraw-Hill, 1970.

Lewis, Douglass G., and Lovett H. Weems, Jr., eds. A Handbook for Seminary Presidents. Grand Rapids, MI: William B. Eerdmans Publishing, 2006.

Lindsay, Allan G. "God Used a Man: Goodloe Bell--Faithful Friend to Right in Every Form." Adventist World -NAD 4, no. 8 (2008): 35.

Linton, Ralph. The Cultural Background of Personality. New York, NY: D. AppletonCentury, 1945.

Loma Linda University. Organization of the University. Loma Linda, CA: Office of the President, 1998.

Loma Linda University. "The Role of President in Contemporary American Universities: A Summary of Findings with Brief Recommendations." Riverside, CA: The Committee, 1979.

Loyola University. "By Laws: Article XIII--Administration." New Orleans, LA: Office of the President, 1997.

Lucas, Christopher J. American Higher Education: A History. $2^{\text {nd }}$ ed. New York, NY: Palgrave Macmillan, 2006.

Maphosa, Norman. "Critical Skills for Chief Executives in Adventist Institutions of Higher Learning." In Christ in the Classroom: Adventist Approaches to the Integration of Faith and Learning, edited by Humbarto M. Rasi, 12:144. Silver Spring, MD: Institute for Christian Teaching, 1994.

Massey, James William. "The Role of the Chief Executive in Universities and Senior Colleges Which Are Members of the Southern Association of Colleges and Schools." Ph.D. thesis, University of Mississippi, 1969.

Mayden, Harry. "Christian Administration: Relationships and Responsibilities." In Christ in the Classroom: Adventist Approaches to the Integration of Faith and Learning, edited by Humberto M. Rasi, 18:113-122. Silver Spring, MD: Institute for Christian Teaching, 1997.

Mayhew, Lewis B. The Smaller Liberal Art College. Washington, DC: Center for Applied Research in Education, 1962. 
McAllister-Wilson, David, Craig Williford, and David Neelands. "The President's Role in Enrollment Management and Student Issues." In $A$ Handbook for Seminary Presidents, edited by G. Douglass Lewis and Lovett H. Weems, Jr., 141-151. Grand Rapids, MI: William B. Eerdmans Publishing, 2006.

McCarter, Neely Dixon. The President as Educator. Atlanta, GA: Scholars Press, 1996.

McMillan, James H., and Sally Schumacher. Research in Education: A Conceptual Introduction. $3^{\text {rd }}$ ed. New York, NY: HarperCollins College Publishers, 1993.

Meacham, Jack. "What's the Use of a Mission Statement?" ACADEME: Bulletin of the American Association of University Professors 94, no. 1 (2008): 21-24.

Millett, John D. The Multiple Roles of the College and University President. Washington, DC: American Council on Education, 1976.

Mitchell, William J. Imaging MIT: Designing a Campus for the Twenty-First Century. Cambridge, MA: The MIT Press, 2007.

Moring, Mark. "So Tell Me What's So Different About a Christian College?" Campus Life (1994): 18.

Muller, Steven. "The Advancement Role in the Research University." In The Advancement President and the Academy: Profiles in Institutional Leadership, edited by Mary Kay Murphy, 49. Phoenix, AZ: Oryx Press, 1997.

Murphy, Joseph, and Karen Seashore Louis, eds. Handbook of Research on Educational Administration: A Project of the American Educational Research Association. $2^{\text {nd }}$ ed. San Francisco, CA: Jossey-Bass, 1999.

Murphy, Mary Kay, ed. The Advancement President and the Academy: Profiles in Institutional Leadership. Phoenix, AZ: Oryx Press, 1997, 49.

Narbarte, Miriam P. "Teaching Human Resources Management: Its Implications for Seventh-day Adventist Educators." In Christ in the Classroom: Adventist Approaches to the Integration of Faith and Learning, edited by Humbarto M. Rasi, 11:97-109. Silver Spring, MD: Institute for Christian Teaching, 1994.

Nason, J. W. Presidential Assessment: A Challenge to College and University Leadership. Washington, DC: Association of Governing Boards of Universities and Colleges, 1980. 
Nelson, Lawrence O. "Role Expectations for Selected College and University

Presidents." Ph.D. dissertation, Michigan State University, 1960.

Neumann, Anna. "The Thinking Team." The Journal of Higher Education 62, no. 5 (1991): 571 .

Northeast Brazil College (SALT). "Structure and Administrative Organization." Cachoeira, BA, Brazil: Office of the President, 1998.

Nudd, Garrett W. "Practical Public Relations: Making Your School Stand Out Despite Limited Financial Resources." The Journal of Adventist Education 64, no. 1 (2001): 9-13.

O'Connell, Thomas Edward. "The Five Roles of the College or University President." Ed.D. dissertation, University of Massachusetts, 1974.

Oliver, Arnold Robert. "The University Presidency as Perceived by Boards of Trustees, Presidents, and Academic Vice Presidents in Selected Colleges and Universities." Ph.D. dissertation, Southern Illinois University, 1972.

Ostar, Roberta H. Public Roles, Private Lives: The Representational Role of College and University Presidents. Washington, DC: American Council on Education, 1991.

Owens, Robert G. Organizational Behavior in School. Englewood Cliffs, NJ: PrenticeHall, 1970.

Paolitto, Julia. "President of Yale, or CEO of Yale, Inc?" www.yaleherald.com/archive/xxxi/2001.04.20/features/front.html (accessed April 2, 2008).

Parsons, Talcott. The Social System. Glencoe, IL: Free Press, 1951, 25.

Parten, Mildred. Surveys, Polls, and Samples. New York, NY: Harper, 1950.

Patterson, James A. Shining Lights: A History of the Council for Christian Colleges and Universities. Grand Rapids, MI: Baker Academic, 2001.

Payton, Robert L. "Presidents as Public Teachers." In The Advancement President and the Academy: Profiles in Institutional Leadership, edited by Mary Kay Murphy, 89. Phoenix, AZ: Oryx Press, 1997.

Penny, Sherry H. "Five Challenges for Academic Leaders in the $21^{\text {st }}$ Century." Educational Record 77, no. 2-3 (1996): 19-22. 
Peruvian Union University (SALT). "Structure and Administrative Organization." Juliaca, Peru: Office of the President, 1998.

Prater, Ralph. The College President. Washington, DC: Center for Applied Research in Education, 1963.

Prince, Gregory S., Jr. Teach Them to Challenge Authority: Educating for Healthy Societies. New York, NY: The Continuum International Publishing Group, 2008.

Rhodes, Frank H. T. "The University at the Millennium: Missions and Responsibilities of Research Universities." In Governance in Higher Education: The University in a State of Flux, edited by Werner Z. Hirsh and Luc E. Weber, 12-13. London, England: Economica, 2001.

Rile, Judith A. "The Changing Role of the President in Higher Education." www.newfoundations.com/OrgTheory/Rile721.html (accessed January 14, 2008).

River Plate Adventist University. Faculty Handbook. Entre Rios, Argentina: Office of President, 1998.

Robbins, Stephens P. Personnel: The Management of Human Resources. Englewood Cliffs, NJ: Prentice Hall, 1987. Quoted in Miriam P. Narbarte, "Teaching Human Resources Management: Its Implications for Seventh-day Adventist Educators." In Christ in the Classroom: Adventist Approaches to the Integration of Faith and Learning, edited by Humbarto M. Rasi, 11:104. Silver Spring, MD: Institute for Christian Teaching, 1994.

Roscoe, Lewis. Planning the Campus: Making No Little Plans for Colleges and Universities. Amherst, MA: L \& A Publications, 2000.

Rosenblith, Suzanne, and Bea Bailey. "Comprehensive Religious Studies in Public Education: Educating for a Religiously Literate Society." Educational Studies 42, no. 2 (2007): 93-109.

Rosso, Henry A., and Eugene R. Tempel. Achieving Excellence in Fund Raising. $2^{\text {nd }}$ ed. San Francisco, CA: Jossey-Bass, 2003.

Rudolph, Frederick. The American College President: A History. Athens, GA: University of Georgia, 1991. 
Ruger, Anthony, John Canary, and Steven Land. "The President's Role in Financial Management." In $A$ Handbook for Seminary Presidents, edited by G. Douglass Lewis and Lovett H. Weems, Jr., 90-111. Grand Rapids, MI: William B. Eerdmans Publishing, 2006.

Saleve Adventist University. Unpublished manuscript. Collonges-sous-Saleve, France: Office of the President, 1998.

Sarbin, Theodore, and Vernon L. Allen. "Role Theory." In Handbook of Social Psychology, edited by Gardner Lindzey and Elliot Aronson. $2^{\text {nd }}$ ed. Reading, MA: Addison-Wesley, 1968.

Schantz, Iris S. "Leadership and Servanthood: Challenges, Tensions and Possibilities in Christian Organizations." In Christ in the Classroom: Adventist Approaches to the Integration of Faith and Learning, edited by Humberto M. Rasi, 7:231-250. Silver Spring, MD: Institute for Christian Teaching, 1993.

Schmidt, George P. The Old Time College President. New York, NY: Columbia University Press, 1930.

Scott, John C. "The Mission of the University: Medieval to Postmodern Transformations." The Journal of Higher Education 77, no. 1 (2006): 1-33.

Scott, William Richard. Organizations: Rational, Natural, and Open Systems. 5th ed. Upper Saddle River, NJ: Prentice-Hall, 2003.

Segovia, Agripino C. "Christian Leadership Initiative: A Critical Factor in School Administration." In Christ in the Classroom: Adventist Approaches to the Integration of Faith and Learning, edited by Humberto M. Rasi, 2:217-236. Silver Spring, MD: Institute for Christian Teaching, 1991.

Seventh-day Adventist Encyclopedia. 1996 ed. Hagerstown, MD: Review and Herald. S.v. "Seventh-day Adventist." "Education, Seventh-day Adventist Philosophy Of."

Seventh-day Adventist Church Manual. $17^{\text {th }}$ ed. Hagerstown, MD: Review and Herald, 2005.

Seventh-day Adventist Yearbook 2006. Hagerstown, MD: Review and Herald, 2006, 435486.

Seventh-day Adventist Yearbook 2008. Hagerstown, MD: Review and Herald, 2008. 
Shand, Herbert Olivier. "Comprehensive Planning in Seventh-day Adventist Colleges and Universities in the United States." Ph.D. dissertation, Loma Linda University, 1987.

Shavelson, R. J. Statistical Reasoning for the Behavioral Sciences. $2^{\text {nd }}$ ed. Boston, MA: Allyn and Bacon, 1988. Quoted in Robert Alan Darden, "Study of the Efficacy of a Christian-Based Inpatient Hospital for Treating Eating Disorders, Depression, and Spiritual Distress." Ph.D. dissertation, Andrews University, 2002.

Silas, Samuel L. "Role Expectations of Presidents in Predominantly Black State and Privately Supported Colleges and Universities as Perceived by Trustees, Presidents, and Deans." Ph.D. dissertation, Southern Illinois University, 1974.

Simmons, Ella. "Aiming Higher." Adventist World-NAD: An International Paper for Seventh-day Adventists 5, no. 7 (2009): 10.

Simon, Herbert A. “The Job of a College President." Educational Record 68 (1967): 6970 ,

Smith, Daryl G, Caroline S. Turner, Nana Osei-Kofi, and Sandra Richards. "Interrupting the Usual: Successful Strategies for Hiring Faculty." The Journal of Higher Education 75, no. 2 (2004): 133-156.

Soshnik, Joseph. "The Campus Presidency." In Perceptions in Public Higher Education, edited by Gene A. Budig, 5. Lincoln, NE: University of Nebraska Press, 1970.

South American Division. Internal Policy. Brasilia DF, Brazil: South American Division Executive Committee, 1998.

Southern Adventist University. Faculty Handbook. Collegedale, TN: University Press, 1999.

Southwestern Adventist University. Faculty Handbook. Keene, TX: University Press, 1999.

Spicer Memorial College. Unpublished manuscript. Pune, India: Office of the President, 1998.

Stogdill, Ralph M., Ellis L. Scott, and William E. Jaynes. Leadership and Role Expectations. Columbus, OH: Bureau of Business Research, College of Commerce and Administration, Ohio State University, 1956.

Stoke, Harold W. The American College President. New York, NY: Harper, 1959. 
Stout, Fern D. "A Comparison of Role Perceptions of Selected University Presidents and Their Reference Groups." Ph.D. dissertation, University of Mexico, 1962.

Stover, Del. "Teacher Recruiting Especially Challenging for Urban School Boards." The Education Digest 73, no. 3 (2007): 29.

Tabachnick, Barbara G., and Linda S. Fidell. Using Multivariate Statistics. Boston, MA: Pearson Education, 2007.

Tarbitt, Val. "Mentoring On-Line: Rethinking the Tutor/Student Experience." In Mentoring in Education: An International Perspective, edited by Cedric Cullingford. Burlington, VT: Ashgate Publishing, 2006.

Tavallali, Rahmat ola. "Role and Functions of Presidents and Chancellors of Selected American Public Universities." Ph.D. dissertation, University of Akron, 1981.

Taylor, Frederick Winslow, and Frank B. Gilbreth. The Principle of Scientific Management, Primer of Scientific Management. Easton, PA: Hive Publishing Co., 1985.

The Stanford Daily Staff. "University Must Rethink the Role of President." www.stanforddaily.com/article/2001/2/9 (accessed January 22, 2008).

Thorpe, David A. College Administration. Boston, MA: Narcisist Press, 1945.

Tiede, David L., David Draper, and Wilson Yates. "The President's Role in Defining Mission and Strategic Planning." In A Handbook for Seminary Presidents, edited by G. Douglass Lewis and Lovett H. Weems, Jr.,60-70. Grand Rapids, MI: William B. Eerdmans Publishing, 2006.

Trimble, Jerry Max. "Priorities of College and University Presidents." Ph.D. dissertation, Boston College, 1973.

Twing, Erwin. The College President. New York, NY: Macmillan, 1939.

Union College. Faculty/Staff Handbook. Lincoln, NE: College Press, 1999.

University of Notre Dame. Unpublished manuscript. Notre Dame, IN: Office of the President, 1998.

University of Washington. "University President's Role in Master Planning." www.washington.edu/community/cmp_site/Plan_Background.html (accessed July 7, 2007). 
Valparaiso University. Unpublished manuscript. Valparaiso, IN: Office of the President, 1998.

Vineyard, Edwin E. The Pragmatic Presidency: Effective Leadership in the Two-Year College. Bolton, MA: Anker Publishing, 1993.

Waier, Phil, Steve Plotner, and Andrea Keenan. Preventive Management for Higher Education Facilities: A Planning and Budgetary Tool for Facilities Professionals. Kingston, MA: R.S. Means, 2002.

Walter, Edwin Carlton. "A History of Seventh-day Adventist Higher Education in the United States." Ph.D. dissertation, University of California, Berkeley, 1996.

Weeks, I. D. "The University President and the Publics." A presidential address at the National Association of State Universities Transactions and Proceedings, 1950.

Westmeyer, Paul. An Analytical History of American Higher Education. $2^{\text {nd }}$ ed. Springfield, IL: Charles C. Thomas, 1997.

White, Ellen G. Education. Boise, ID: Pacific Press, 1952. Testimonies for the Church. Vol. 4. Boise, ID: Pacific Press, 1948.

Wilch, Leroy A. "Perceived Leadership Role and Function of Nebraska Community College Presidents and Campus Directors." Ph.D. dissertation, University of South Dakota, 1986.

Wileman, Gwen. "The Challenge of Strategic Leadership." In Strategic Leadership of Change in Higher Education, edited by Stephanie Marshall, 174-175. New York, NY: Routledge, 2007.

Wiseman, L. "The University President: Academic Leadership in an Era of Fundraising and Legislative Affairs." In Managing Institutions of Higher Education Into the $21^{s t}$ Century, edited by Ronald R. Sims and Serbrenia J. Sims. Westport, CT: Greenwood Press, 1991.

Zigarmi, Andrea. "The Role of the University President as a Boundary Person: A Case Study." Ph.D. dissertation, University of Massachusetts, 1974.

Zimmerman, Robert M., and Ann W. Lehman. Board That Love Fundraising: A How-toGuide for Your Board. San Francisco, CA: Jossey-Bass, 2004. 
Zwemer, Tom. "Candor and Civility: A Post-9/11 Review of Academic Freedom." Liberty: A Magazine of Religious Freedom 103, no. 2 (2008): 3-5. 
VITA 
VITA

Name: $\quad$ Sunith Kumar Das

4775 Kimber Lane, Berrien Springs, MI 49103

Undergraduate and Graduate Schools Attended:

Andrews University, Berrien Springs, MI

Adventist International Institute of Advanced Studies, Philippines

Dhaka University, Dhaka, East Pakistan

Jagannath University, Dhaka, East Pakistan

\section{Education:}

2009 Ph.D., Educational Administration and Supervision

School of Education, Andrews University, Berrien Springs, MI

1994 M.A., Educational Administration and Supervision

School of Education, Andrews University, Berrien Springs, MI

1968 M.A., Philosophy

Faculty of Arts, Dhaka University, Dhaka, East Pakistan

1965 B.A., English

Jagannath University, Dhaka, East Pakistan

\section{Professional Experience:}

2006 - present Director

Samaritan Services, a non-profit initiative committed to educational and spiritual development among target groups

2005 - present Chaplain

Lakeland Regional Health System, Berrien County, MI 
Net 98 New Millennium world satellite evangelism, reaching Banglaspeaking people in India and Bangladesh

1994 - present Senior Pastor.

South Asia SDA Church on the campus of Andrews University

Berrien Springs, MI

1992 - 2004 Director

Freshman \& College Seminars (for academic probationary students)

Andrews University, Berrien Springs, MI

Faculty

College of Technology, Andrews University, Berrien Springs, MI

1986 - 1987 Administrative Fellow

Far Eastern Division of SDA, Singapore

1987 - 1992 Assistant Director

Research \& Planning, Far Eastern Division of SDA, Singapore

Vice Secretary

Global Mission, Far Eastern Division of SDA, Singapore

Coordinator

Retirees' Association, Far Eastern Division of SDA, Singapore

1981 - 1986 Union Executive Secretary

Bangladesh Union Mission of SDA, Dhaka, Bangladesh

1976 - 1986 Education Director

Bangladesh Union Mission of SDA, Dhaka, Bangladesh

1973 - 1975 President

Bangladesh Adventist Seminary

Vill. Gowalbathan, P.O. Kaliakoir, Dist. Gazipur, Bangladesh

1970 - 1972 Registrar, Librarian, and English Teacher

Kellogg-Mookerjee Memorial Seminary

Vill. Jalirpar, P.O. Takerhat, Dist. Gopalgonj, Bangladesh

1968 - 1970 Translator

Sabbath school lessons, week of prayer readings, Voice of Prophecy Bible

Correspondence School's health lessons, and Faith for Today lectures

East Pakistan Mission of Seventh-day Adventists, Dhaka, East Pakistan 